\title{
Solution Methods for Multi-Objective Robust Combinatorial Optimization
}

\author{
Dissertation \\ zur Erlangung des mathematisch-naturwissenschaftlichen Doktorgrades \\ „Doctor rerum naturalium“ \\ der Georg-August-Universität Göttingen \\ im Promotionsprogramm „PhD School of Mathematical Sciences“ (SMS) \\ der Georg-August University School of Science (GAUSS)
}

vorgelegt von

Lisa Thom

aus Kassel

Göttingen, 2018 


\section{Betreuungsausschuss}

Prof. Dr. Anita Schöbel, Institut für Numerische und Angewandte Mathematik, Georg-August-Universität Göttingen

Jun.-Prof. Dr. Anja Fischer, Juniorprofessur Management Science, Technische Universität Dortmund

\section{Mitglieder der Prüfungskommission}

Referentin: Prof. Dr. Anita Schöbel, Institut für Numerische und Angewandte Mathematik, Georg-August-Universität Göttingen

Korreferentin: Dr. Marie Schmidt, Department of Technology and Operations Management, Erasmus University Rotterdam

\section{Weitere Mitglieder der Prüfungskommission:}

Jun.-Prof. Dr. Anja Fischer, Juniorprofessur Management Science, Technische Universität Dortmund

Prof. Dr. Gerlind Plonka-Hoch, Institut für Numerische und Angewandte Mathematik, Georg-August-Universität Göttingen

Prof. Dr. Dominic Schuhmacher, Institut für Mathematische Stochastik, GeorgAugust-Universität Göttingen

Prof. Dr. Stephan Waack, Institut für Informatik, Georg-August-Universität Göttingen

Tag der mündlichen Prüfung: 19. April 2018 


\section{Contents}

$\begin{array}{ll}\text { 1. Motivation } & 1\end{array}$

2. Preliminaries and Related Literature 3

2.1. Multi-Objective Optimization . . . . . . . . . . . . . . . 3

2.2. Robust Optimization . . . . . . . . . . . . . . . 5

2.3. Multi-Objective Robust Optimization . . . . . . . . . . . . 7

3. Summary of the Publications $\mathbf{1 5}$

3.1. Multi-Objective Minmax Robust Combinatorial Optimization with Cardinality-Constrained Uncertainty . . . . . . . . . . . . 15

3.2. Extensions of Labeling Algorithms for Multi-Objective Uncertain Shortest Path Problems . . . . . . . . . . . . . . . . . 22

3.3. Min-Ordering and Max-Ordering Scalarization Methods for Multi-Objective Robust Optimization . . . . . . . . . . . . . . . . . . . 29

4. Discussion 35

5. Conclusion and Future Work 39

$\begin{array}{ll}\text { Bibliography } & 41\end{array}$

A. Publications $\quad \mathbf{4 9}$

A.1. Multi-Objective Minmax Robust Combinatorial Optimization with Cardinality-Constrained Uncertainty . . . . . . . . . . . . . . . . . 49

A.2. Extensions of Labeling Algorithms for Multi-Objective Uncertain Shortest Path Problems . . . . . . . . . . . . . . . 65

A.3. Min-Ordering and Max-Ordering Scalarization Methods for Multi-Objective Robust Optimization . . . . . . . . . . . . . . . . 110 



\section{Motivation}

Applying classical optimization methods to real-world problems does not always yield the desired result. Two of the main difficulties are that often various (conflicting) objectives are relevant for the same problem and that not all parameters of a model can be predicted accurately in advance.

In many situations one does not pursue only one objective but has to balance several goals, which usually contradict each other: the best solution with respect to one criterion is rarely optimal considering all other criteria. For example, when driving on a road network and choosing between different routes, one might want to minimize travel time, fuel consumption and toll costs at the same time. However, the fastest route is rarely the most economical one regarding fuel consumption, and it is also more likely to contain toll roads.

This contradiction is sometimes resolved by assigning a weight to each criterion and optimizing the sum of the weighted objective functions. However, it is not always easy or even possible to find suitable weights in advance: to obtain an improvement in one objective, some impairment in another objective might or might not be tolerable, depending on the precise values. For example, to accept a 30 minutes delay in order to save some amount of toll cost might be a totally different consideration for an undelayed travel time of 15 minutes versus one of 15 hours.

On the other hand, given two routes with identical fuel consumption and toll cost, surely the faster one will be chosen, regardless of how the decision maker values the objectives. Therefore, in multi-objective optimization, one optimizes over a vector of objective functions instead of a single value. All solutions that cannot be improved in one objective without impairing another objective are of interest. They are called (Pareto) efficient solutions.

Furthermore, an obstacle often encountered when applying optimization methods in practice is missing information. Not all parameters of a model can be stated exactly in advance, in particular when predicting future developments. For example, when choosing a route in a road network, one cannot precisely predict the travel time and fuel consumption, because of potential traffic congestion, red traffic lights, weather conditions etc.

Uncertain problems can be tackled in several ways. To what extent perturbations in the parameters influence a given solution is analyzed by means of sensitivity analysis. In stochastic optimization the expected value, the variation or some other indicator based on the probability distribution is optimized, assuming that enough information on the probability of the various realizations of the data is given. Robust optimiza- 
tion, on the other hand, hedges against (all) possible realizations of the uncertain data, called scenarios. For this purpose, information on possible scenarios but no probability information is assumed. For example, the range of the parameter values can be given as an interval: we might know that driving along a particular route takes between 15 and 30 minutes, but we don't know the expected travel time and variation. The information about the uncertain values can also be given in form of several distinct scenarios, e.g., weather scenarios or other events, which influence the traffic on some or all of the routes.

Intuitively, hedging against all scenarios means hedging against the worst case. Consequently, it is common to optimize the worst case objective value. For example, if an uncertain travel time is to be minimized, one chooses the tour whose worst possible duration is shortest. Nevertheless, there are also other interpretations of robustness, for example minimizing the worst case regret, where, given a specific scenario, the regret is the difference between the objective value of the chosen solution and the best possible objective value for this scenario.

Many real-world problems, as the problem of choosing a route, which we introduced above, do not yield only one but both of these obstacles. Imagine you want to choose a holiday destination and your objectives are the price, the time to get there and the activities you can take part in. The possible activities may depend on the weather, the travel time on traffic congestion or train delays and the price on foreign exchange rates or fuel costs. Another example occurs in the wood industry: Cutting a trunk into boards, one aims to maximize the revenue and minimize the waste. Both depend on the location of the core and damaged parts of the wood, which cannot be determined exactly from the outside, but only after the trunk has been cut.

The optimization problems considered in this thesis are combinatorial problems with multiple objectives and uncertain input parameters. We use concepts from the recently developed field of multi-objective robust optimization, which combines aspects of both multi-objective and robust optimization. Even though several concepts to define so-called robust efficient solutions have been developed during the last years, solution approaches are still rare. In this cumulative thesis, that is, in the underlying publications, we develop models and solution approaches for multi-objective robust combinatorial optimization problems based on techniques from both multi-objective and robust optimization.

In Chapter 2 we introduce concepts and methods of robust and multi-objective optimization as well as multi-objective robust optimization, including a brief literature review. The publications that constitute the cumulative part of this thesis are summarized in Chapter 3, followed by a discussion of the results in Chapter 4 . The conclusion in Chapter 5 contains a summary of the results and potential aspects of future work. 


\section{Preliminaries and Related Literature}

In this chapter, we introduce basic concepts and notations from multi-objective, robust and multi-objective robust optimization, and present related work.

In each of the sections we also devote one paragraph to combinatorial optimization within the scope of the respective field. In a combinatorial optimization problem, a set of elements $E$ and a cost for each element is given, as well as a set of feasible subsets of $E$. Usually, the aim is to find a feasible subset, such that the sum of the contained elements' costs is minimal. An example is the shortest path problem, where $E$ is the edge set in a graph and the feasible set consists of all simple paths between two given nodes.

Throughout the thesis we use the symbols $<$ (smaller than) and $\leqq$ (smaller than or equal to) to compare values in $\mathbb{R}$, in order to be consistent with the notation for comparing vectors, which we introduce in the next section (Definition 2.2). We write $A_{(i, \cdot)}$ for the $i$-th row of a matrix $A$ and $A_{(\cdot, i)}$ for its $i$-th column. The transpose of a vector or matrix $A$ is denoted by $A^{T}$.

Furthermore, we use a [./.] notation to maintain a concise text: instead of writing "a feasible solution $x$ is optimal if $z(x) \leqq z(y)$ for every feasible solution $y \neq x$ and uniquely optimal if $z(x)<z(y)$ for every feasible solution $y \neq x$ " we write "a feasible solution $x$ is [ / uniquely] optimal, if $z(x)[\leqq /<] z(y)$ for every feasible solution $y \neq x "$

\subsection{Multi-Objective Optimization}

The foundations of multi-objective optimization, also called multi-criteria optimization, were laid at the end of the 19th century by Edgeworth (1881) and Pareto (1896). For a recent textbook on the topic we refer to Ehrgott (2005). In order to optimize several (scalar-valued) objective functions simultaneously, each feasible solution is assigned an objective vector instead of a scalar objective value.

Definition 2.1. Given a set $\mathcal{X}$ of feasible solutions and $k \in \mathbb{N}$ scalar-valued objective 
functions $z_{1}, \ldots, z_{k}: \mathcal{X} \rightarrow \mathbb{R}$, we call

$$
\min _{x \in \mathcal{X}} z(x)=\left(\begin{array}{c}
z_{1}(x) \\
\vdots \\
z_{k}(x)
\end{array}\right)
$$

a multi-objective optimization problem (MOP). For $k=1$ we obtain a singleobjective optimization problem.

For $k \geqq 2$, a solution that minimizes all objectives at once does usually not exist. Therefore, we use the following relation to compare two vectors and to define efficient solutions, following the notation in Ehrgott (2005).

Definition 2.2. Let $k \in \mathbb{N}$. For two vectors $y^{1}, y^{2} \in \mathbb{R}^{k}$ we use the notation

$$
\begin{aligned}
& y^{1}<y^{2} \Leftrightarrow y_{i}^{1}<y_{i}^{2} \text { for all } i \in\{1, \ldots, k\}, \\
& y^{1} \leq y^{2} \Leftrightarrow y_{i}^{1} \leqq y_{i}^{2} \text { for all } i \in\{1, \ldots, k\} \text { and } y^{1} \neq y^{2}, \\
& y^{1} \leqq y^{2} \Leftrightarrow y_{i}^{1} \leqq y_{i}^{2} \text { for all } i \in\{1, \ldots, k\} .
\end{aligned}
$$

We also define the cones $\mathbb{R}_{[>/ \geq / \geqq]}^{k}:=\left\{y \in \mathbb{R}^{k}: 0[</ \leq / \leqq] y\right\}$.

By means of the relations in Definition 2.2 we define (Pareto) efficient solutions, which cannot be improved in one objective without worsening them in another objective, and the closely related concepts of weakly and strictly efficient solutions.

Definition 2.3. A solution $x \in \mathcal{X}$ is a [weakly/./strictly] efficient solution for $M O P$, if there does not exist any feasible solution $x^{\prime} \in \mathcal{X}, x^{\prime} \neq x$ with $z\left(x^{\prime}\right)[</ \leq / \leqq] z(x)$. Then $z(x)$ is called [weakly/./strictly] nondominated. A complete set of efficient solutions is a set $\mathcal{X}^{\prime} \subseteq \mathcal{X}$ such that for every efficient solution $x$ there exists $x^{\prime} \in \mathcal{X}^{\prime}$ with $z(x)=z\left(x^{\prime}\right)$.

Note that a solution $x \in \mathcal{X}$ is [weakly/./strictly] efficient if and only if there is no $x^{\prime} \in \mathcal{X}$ with $x^{\prime} \neq x$ and

$$
z\left(x^{\prime}\right) \in z(x)-\mathbb{R}_{[>/ \geq / \geqq]}^{k}
$$

In contrast to single-objective optimization, where the optimal objective value is unique, there often exist many nondominated objective vectors if $k \geqq 2$. A common approach to find efficient solutions are scalarization methods: by solving a family of single-objective so-called scalarized problems, whose solutions are efficient for the multi-objective problem, one finds a set of solutions with several different (and possibly all) nondominated objective vectors. Ehrgott (2006) gives an overview on popular scalarization methods, among them the weighted sum method (e.g., Gass and Saaty, 1955), the $\epsilon$-constraint method (Haimes et al., 1971; Chankong and Haimes, 1983) and the weighted Chebychev method (Bowman, 1976; Steuer and Choo, 1983). 


\section{Multi-Objective Combinatorial Optimization}

Many combinatorial optimization problems have been extended to multi-objective combinatorial problems. An overview on multi-objective combinatorial optimization is given by Ehrgott and Gandibleux (2000) and Ehrgott (2005) among others. Often, there exist instances with exponentially many nondominated objective vectors, see, e.g., Hansen (1980) for the shortest path problem and Hamacher and Ruhe (1994) for the minimum spanning tree problem. Nevertheless, algorithms for solving particular single-objective combinatorial optimization problems can sometimes be extended to find all nondominated objective vectors of the multi-objective problem. For example, extensions of the famous labeling algorithms by Dijkstra (1959) and Bellman, Ford and Moore (e.g., Bellman, 1958) have been developed to solve the multi-objective shortest path problem (see, e.g., Martins, 1984; Corley and Moon, 1985; Paixão and Santos, 2013).

\subsection{Robust Optimization}

Robust optimization is one way to handle uncertain parameters in an optimization problem. No probability data is needed, but the potential realizations of the uncertain data are assumed to be given via an uncertainty set $\mathcal{U}$, which contains all possible scenarios.

In this thesis, if the feasible set of the optimization problem is subject to uncertainty, we aim to find solutions which are feasible for all scenarios, following seminal works on robustness, e.g., Soyster (1973) and Ben-Tal and Nemirovski (1998). For this purpose, the sets of feasible solutions under all scenarios can be intersected in advance to obtain a set of robust feasible solutions. Hence, in the following, we assume the feasible set $\mathcal{X}$ to be deterministic, which means that it is not subject to uncertainty, and define an uncertain optimization problem with uncertainty in the objective function only. Nevertheless, we also mention robustness concepts that do not inherently make this assumption.

Definition 2.4. Given a feasible set of solutions $\mathcal{X}$, an uncertainty set $\mathcal{U}$, and an objective function $z: \mathcal{X} \times \mathcal{U} \rightarrow \mathbb{R}$, the family $(\mathcal{P}(\xi), \xi \in \mathcal{U})$ of optimization problems

$$
\mathcal{P}(\xi) \quad \min _{x \in \mathcal{X}} z(x, \xi)
$$

is called an uncertain optimization problem (UP). A problem that is not subject to uncertainty, e.g. UP with $|\mathcal{U}|=1$, is called deterministic.

Several robustness concepts have been developed to define robust solutions for UP. One of the most popular is minmax robustness, first introduced by Soyster (1973) and extensively studied, e.g., by Ben-Tal et al. (2009). A minmax robust optimal solution is a solution with minimal objective value in the worst case, i.e., it solves 
the following deterministic problem, called the minmax robust counterpart of the uncertain problem.

Definition 2.5. Let an uncertain optimization problem UP be given. A solution $x \in \mathcal{X}$ is minmax robust optimal for UP, if it is optimal for the deterministic problem

$$
\min _{x \in \mathcal{X}} \sup _{\xi \in \mathcal{U}} z(x, \xi)
$$

which is called minmax robust counterpart.

Other robustness concepts include deviation robustness (see Kouvelis and Yu, 1997), also called minmax regret robustness. Here, the maximal regret over all scenarios is minimized, which is the difference between the objective value of the respective solution and the optimal objective value for this scenario. If the optimal value is additionally used as a scaling factor for the regret, one obtains relative robustness (see Kouvelis and $\mathrm{Yu}, 1997$ ). Lightly robust solutions (Fischetti and Monaci, 2009; Schöbel, 2014) are required to be not too bad in the most likely case, called nominal scenario. Adjustable robustness (Ben-Tal et al., 2004) or recoverable robustness (Cicerone et al., 2007; Liebchen et al., 2009; Erera et al., 2009) is used if part of the chosen solution can be determined or changed after the realization of the uncertain data. For an overview on robustness concepts see, e.g., Goerigk and Schöbel (2016).

Another approach to consider all scenarios at once, which we refer to as multi-scenario optimality, is inspired by (Pareto) efficiency in multi-objective optimization: one aims to find solutions which cannot be improved for one scenario without worsening them for another scenario. For the relationship between multi-scenario optimality and several robustness concepts see, e.g., Klamroth et al. (2017). Iancu and Trichakis (2014) combine multi-scenario efficiency and minmax robustness to define Pareto robust optimal solutions, which are both minmax robust optimal and multi-scenario optimal.

Apart from the robustness concept, the uncertainty set, too, plays an important role regarding the obtained solutions and the complexity of the robust problem. A finite uncertainty set contains a finite number of scenarios. In case of interval uncertainty the uncertain parameters vary independently of each other between given lower and upper bounds. Further common uncertainty sets include ellipsoidal and polyhedral uncertainty sets. Bertsimas and Sim (2003) introduced bounded uncertainty, also called cardinality-constrained, budgeted, banded or $\Gamma$-uncertainty (see also Bertsimas and Sim, 2004). They assume that the uncertain parameters vary independently of each other in given intervals, but not all of them deviate from their nominal value, which we assume here to be their minimal value.

Definition 2.6. Let an uncertain optimization problem with $n \in \mathbb{N}$ uncertain parameters be given, with a nominal value $\hat{c}_{j} \in \mathbb{R}$ and an interval length $\delta_{j} \in \mathbb{R}_{\geqq}$for each 
uncertain parameter $c_{j}$, where $j \in\{1, \ldots, n\}$. Further, let $\Gamma \in \mathbb{Z}$ with $0 \leqq \Gamma \leqq n$ be given. We define the bounded uncertainty set as

$$
\mathcal{U}^{b}:=\left\{c \in \mathbb{R}^{n}: c_{j}=\hat{c}_{j}+\beta_{j} \delta_{j}, \beta_{j} \in[0,1] \forall j \in\{1, \ldots, n\}, \sum_{j=1}^{n} \beta_{j} \leqq \Gamma\right\}
$$

Variations and extensions of bounded uncertainty have been developed, e.g., by Poss (2014) and Büsing and D'Andreagiovanni (2014).

Chassein et al. (2018) assume that the uncertainty set is determined based on a discrete sample of scenarios and experimentally investigate how different kinds of uncertainty sets influence the obtained minmax robust optimal solutions.

\section{Robust Combinatorial Optimization}

Robust combinatorial optimization problems have been investigated extensively, in particular with discrete and interval uncertainty, see, for example, Kouvelis and $\mathrm{Yu}$ (1997) and the recent survey by Kasperski and Zieliński (2016). When considering uncertainty in the objective function, the uncertain parameters are the costs of the elements. With discrete uncertainty, minmax robust counterparts of several polynomially solvable problems have been proven to be NP-hard, including the shortest path problem, the minimum spanning tree problem and the assignment problem (Murthy and Her, 1992; Kouvelis and $\mathrm{Yu}, 1997$ ). If the costs of the elements vary independently of each other, e.g., in intervals, the minmax robust counterpart can be reduced to a deterministic problem by only considering the maximal cost of each element. For bounded uncertainty, Bertsimas and Sim (2003) have developed an algorithm to solve the minmax robust counterpart in polynomial time, provided that the underlying deterministic problem is polynomially solvable.

\subsection{Multi-Objective Robust Optimization}

The examples in Chapter 1 show that it is not uncommon for a real-world problem to be of multi-objective nature and to contain uncertain parameters, resulting in a multi-objective uncertain optimization problem.

Definition 2.7. Given a feasible set of solutions $\mathcal{X}$, an uncertainty set $\mathcal{U}$, and a multi-objective function $z: \mathcal{X} \times \mathcal{U} \rightarrow \mathbb{R}^{k}$, the family $(M O P(\xi), \xi \in \mathcal{U})$ of deterministic multi-objective optimization problems

$$
\operatorname{MOP}(\xi) \quad \min _{x \in \mathcal{X}} z(x, \xi)
$$

is called a multi-objective uncertain optimization problem (MOUP). 
Remark 2.8. Throughout this thesis we assume $\mathcal{X}$ and $\mathcal{U}$ to be compact and nonempty and the $z_{i}$ to be continuous in $x$ and $\xi$. In this case $\max _{\xi \in \mathcal{U}} z_{i}(x, \xi)$ exists for all $i \in\{1, \ldots, k\}$ and $x \in \mathcal{X}$.

The field of multi-objective robust optimization, combining concepts and methods from robust and multi-objective optimization, has for the most part been developed during the last years and is currently gaining more and more interest. For a recent survey on multi-objective robust optimization see Wiecek and Dranichak (2016).

\section{Robustness Concepts for Multi-Objective Optimization}

Similar to single-objective robust optimization, several robustness concepts for multiobjective optimization have been introduced, which define robust efficient solutions for multi-objective uncertain optimization problems.

An intuitive approach to define robust efficient solutions for a multi-objective uncertain optimization problem is to choose solutions that are efficient for each scenario. It was first proposed by Bitran (1980) for linear problems with interval uncertainty and is often referred to as necessary efficiency. In terms of multi-objective robust optimization, it was established as highly robust efficiency by Kuhn et al. (2016) and Ide and Schöbel (2016).

Definition 2.9. A solution $x \in \mathcal{X}$ is highly robust efficient for MOUP if

$$
\forall \xi \in \mathcal{U} \nexists x^{\prime} \in \mathcal{X}: z\left(x^{\prime}, \xi\right) \leq z(x, \xi) .
$$

However, there is no guarantee that a highly robust efficient solution exists. Bitran (1980) propose a second reasonable criterion, often referred to as possible efficiency: the chosen solutions should be efficient for at least one of the scenarios. This concept is identical to flimsily robust efficiency by Kuhn et al. (2016) and Ide and Schöbel (2016).

Definition 2.10. A solution $x \in \mathcal{X}$ is flimsily robust efficient for MOUP if

$$
\exists \xi \in \mathcal{U} \nexists x^{\prime} \in \mathcal{X}: z\left(x^{\prime}, \xi\right) \leq z(x, \xi) .
$$

An extension of the single-objective concept of minmax robustness to multi-objective optimization was introduced by Kuroiwa and Lee (2012) (see also Fliege and Werner, 2014). They consider the worst case in each objective independently and search efficient solutions for the resulting deterministic multi-objective problem.

Definition 2.11. Given a multi-objective uncertain optimization problem, we define

$$
\bar{z}(x):=\left(\begin{array}{c}
\max _{\xi \in \mathcal{U}} z_{1}(x, \xi) \\
\vdots \\
\max _{\xi \in \mathcal{U}} z_{k}(x, \xi)
\end{array}\right) .
$$


$A$ solution $x \in \mathcal{X}$ is point-based minmax robust [weakly $/ \cdot /$ strictly] efficient for MOUP, if it is a [weakly/./strictly] efficient solution for the multi-objective deterministic robust counterpart $\min _{x \in \mathcal{X}} \bar{z}(x)$, i.e., if there is no $x^{\prime} \in \mathcal{X}$ with $x^{\prime} \neq x$ and

$$
\bar{z}\left(x^{\prime}\right) \in \bar{z}(x)-\mathbb{R}_{[>/ \geq / \geqq]}^{k}
$$

In the following, we abbreviate point-based minmax robust to pointMR.

This concept has been extensively applied, e.g., to portfolio optimization (Fliege and Werner, 2014), game theory (Yu and Liu, 2013) and the planning of sustainable supply chains (Hombach et al., 2017). Krüger et al. (2017) introduce the notion of a robustness gap for this concept, which measures what is lost by implementing a robust efficient solution instead of an efficient solution for a single scenario (see also Krüger, 2018a).

Since, in the concept of point-based minmax robust efficiency, the worst case is considered in each objective independently, the resulting worst case point can be arbitrarily far from the objective vectors obtained by evaluating each scenario. In contrast, the concept of set-based minmax robust efficiency (Ehrgott et al., 2014), takes the dependencies between the objectives into account by comparing the sets of objective vectors obtained for all scenarios (see also Avigad and Branke, 2008).

Definition 2.12. Given a multi-objective uncertain optimization problem, we define the outcome set of a solution $x \in \mathcal{X}$ as

$$
z_{\mathcal{U}}(x):=\{z(x, \xi): \xi \in \mathcal{U}\}
$$

A solution $x \in \mathcal{X}$ is set-based minmax robust [weakly $/ \cdot /$ strictly] efficient for $M O U P$, if there exists no $x^{\prime} \in \mathcal{X}$ with $x^{\prime} \neq x$ and

$$
z_{\mathcal{U}}\left(x^{\prime}\right) \subseteq z_{\mathcal{U}}(x)-\mathbb{R}_{[>/ \geq / \geq]}^{k}
$$

In the following, we abbreviate set-based minmax robust to setMR.

This concept has been applied, e.g., to a veneer cutting problem (Ide et al., 2015) and the design of distributed energy supply systems (Majewski et al., 2017). Ide et al. (2014) generalize it to other cones than $\mathbb{R}_{[>/ \geq / \geq]}^{k}$.

Note that for $k=1$ setMR efficiency and pointMR efficiency reduce to the singleobjective concept of minmax robustness. Ehrgott et al. (2014) show the following connections between the two multi-objective concepts.

Lemma 2.13 (Ehrgott et al. (2014)). Every pointMR [strictly/weakly] efficient solution is also setMR [strictly/weakly] efficient. In case of objective-wise uncertainty, 
i.e., if the uncertainty set can be written as $\mathcal{U}=\mathcal{U}_{1} \times \mathcal{U}_{2} \times \ldots \times \mathcal{U}_{k}$ and the uncertain problem as

$$
\left(\min _{x \in \mathcal{X}}\left(\begin{array}{c}
z_{1}\left(x, \xi_{1}\right) \\
\vdots \\
z_{k}\left(x, \xi_{k}\right)
\end{array}\right), \xi_{i} \in \mathcal{U}_{i} \forall i \in\{1, \ldots, k\}\right),
$$

the sets of pointMR [weakly/·/strictly] efficient solutions and setMR [weakly/./strictly] efficient solutions are identical.

The concepts of convex hull efficiency by Bokrantz and Fredriksson (2017) and properly robust efficiency by Kuroiwa and Lee (2012) are also based on the idea of minmax robustness. Other single-objective robustness concepts have also been transferred to multi-objective optimization, see Kuhn et al. (2016) and Ide and Schöbel (2016) for an extension of light robustness and Nikulin et al. (2013) for an extension of relative robustness.

Further concepts, including those by Gunawan and Azarm (2005); Deb and Gupta (2006); Witting et al. (2013), are also often called robustness concepts for multiobjective optimization, even though they do not follow the classical concepts of single-objective robust optimization and are sometimes more related to sensitivity analysis or stochastic optimization.

Botte and Schöbel (2016) consider a generalization of multi-scenario optimality and Pareto robust optimal solutions to the multi-objective case (see also Wiecek et al., 2009; Kuhn et al., 2016). In case of finitely many scenarios, they define multi-scenario efficient solutions as the efficient solutions to a deterministic multi-objective problem with one objective for each combination of a scenario and an original objective of the uncertain problem.

Definition 2.14. Given a multi-objective uncertain optimization problem with finite uncertainty set $\mathcal{U}=\left\{\xi_{1}, \ldots, \xi_{m}\right\}$, a solution $x \in \mathcal{X}$ is multi-scenario efficient for MOUP if it is an efficient solution for

$$
\min _{x \in \mathcal{X}}\left(\begin{array}{c}
z_{1}\left(x, \xi_{1}\right) \\
\vdots \\
z_{1}\left(x, \xi_{m}\right) \\
z_{2}\left(x, \xi_{1}\right) \\
\vdots \\
z_{2}\left(x, \xi_{m}\right) \\
z_{3}\left(x, \xi_{1}\right) \\
\vdots \\
z_{k}\left(x, \xi_{m}\right)
\end{array}\right) .
$$

For an overview on different robustness concepts for multi-objective optimization we refer to Ide and Schöbel (2016) and Wiecek and Dranichak (2016). 


\section{Scalarization Methods for Multi-Objective Minmax Robust Optimization}

To find pointMR efficient solutions, scalarization methods for multi-objective deterministic problems can be applied to the robust counterpart (see, e.g., Hassanzadeh et al., 2013; Kuroiwa and Lee, 2012; Fliege and Werner, 2014). In case of set-based minmax robust efficiency, the extension of scalarization methods is not as straightforward. Several methods to find setMR efficient solutions based on scalarizations have been developed. Ehrgott et al. (2014) introduce extensions of the weighted sum scalarization method and the $\epsilon$-constraint method, which find setMR weakly efficient solutions. They show that the two methods do not always find the same solutions and that there can exist setMR efficient solutions, which cannot be found by either of these methods. A method based on the (augmented) weighted Chebyshev scalarization for finding setMR weakly efficient solutions has been introduced by Ide (2014). Bokrantz and Fredriksson (2017) consider order-preserving scalarizing functions $s: \mathbb{R}^{k} \rightarrow \mathbb{R}$ and the resulting scalarized problems $\min _{x \in \mathcal{X}} \max _{\xi \in \mathcal{U}} s(z(x, \xi))$. They show that for so-called strongly increasing scalarizing functions the solutions for the scalarized problem are setMR efficient. In an application they consider weighted $p$-norms as scalarizing functions, of which the weighted sum scalarization is a special case.

Schmidt et al. (2018) introduce the min-ordering and the max-ordering method, where a weighted minimum or maximum function is used as scalarizing function. That article is part of this thesis (see Addendum A.3) and is summarized in Section 3.3.

\section{Uncertainty Sets}

Finite and interval uncertainty sets have a straightforward equivalent in the multiobjective case. The idea of bounded uncertainty, however, can be extended to multiple objectives in different ways. It has first been extended to multi-objective problems with uncertainty only in the constraints (Doolittle et al., 2012). Hassanzadeh et al. (2013) consider an objective-wise uncertain linear problem with bounded uncertainty in each objective, i.e., with the following uncertainty set.

Definition 2.15. Let a multi-objective uncertain optimization problem with $n \in \mathbb{N}$ uncertain parameters $\left\{c_{i, 1}, \ldots c_{i, n}\right\}$ in each objective function $z_{i}$ be given. Further, let a nominal value $\hat{c}_{i, j} \in \mathbb{R}$ and an interval length $\delta_{i, j} \in \mathbb{R}_{\geqq}$for each uncertain parameter $c_{i, j}$ be given as well as $k$ numbers $\Gamma_{1}, \ldots, \Gamma_{k} \in \mathbb{Z}$ with $0 \leqq \Gamma_{i} \leqq n \forall i \in\{1, \ldots, k\}$. We define the objective-wise bounded uncertainty set as

$$
\begin{array}{r}
\mathcal{U}^{\text {owb }}:=\left\{c \in \mathbb{R}^{k \times n}: c_{i, j}=\hat{c}_{i, j}+\beta_{i, j} \delta_{i, j}, \beta_{i, j} \in[0,1] \forall i \in\{1, \ldots, k\}, j \in\{1, \ldots, n\},\right. \\
\left.\sum_{j \in\{1, \ldots, n\}} \beta_{i, j} \leqq \Gamma_{i} \forall i \in\{1, \ldots, k\}\right\} .
\end{array}
$$


Schmidt et al. (2018) introduce another extension of bounded uncertainty, where they restrict the total number of uncertain parameters deviating from their nominal value, instead of regarding the objectives independently (see Addendum A.3 and its summary in Section 3.3).

\section{Other Sources of Uncertainty}

In this thesis, we only consider uncertainty arising from uncertain parameter values. However, there exist other possible reasons for uncertainty in optimization. Eichfelder et al. (2017) consider multi-objective optimization problems with decision uncertainty (see also Krüger, 2018a), which occurs when the decision variables cannot be implemented with accuracy. This concept is applied to a problem from agriculture in Krüger et al. (2018); Krüger (2018b). Doolittle et al. (2016) consider uncertainty arising when a scalarization method and scalarizing parameters are chosen in order to solve a deterministic multi-objective optimization problem. The survey by Wiecek and Dranichak (2016) contains an overview on sources of uncertainty in multi-objective optimization.

\section{Multi-Objective Robust Combinatorial Optimization}

An instance of a multi-objective uncertain combinatorial optimization problem (MOUCO) is given by a finite set $E=\left\{e_{1}, \ldots, e_{n}\right\}$, a feasible set $\mathcal{Q}$ containing subsets of $E$, and an uncertainty set $\mathcal{U} \subseteq \mathbb{R}^{k \times n}$ containing all possible element costs: for every $c \in \mathcal{U}, c_{i, j}$ is the cost of element $e_{j}$ w.r.t. the $i$-th objective.

One usually aims to minimize the sum of the contained elements' costs, i.e., MOUCO is the family $(\operatorname{MOCO}(c), c \in \mathcal{U})$ of multi-objective deterministic combinatorial problems

$$
\operatorname{MOCO}(c) \quad \min _{q \in \mathcal{Q}} z(q, c) \quad \text { with } z(q, c):=\left(\begin{array}{c}
\sum_{e_{j} \in q} c_{1, j} \\
\vdots \\
\sum_{e_{j} \in q} c_{k, j}
\end{array}\right)
$$

Alternatively, the set of feasible solutions can be written as a set of binary vectors $\mathcal{X} \subseteq\{0,1\}^{n}$, where each $x \in \mathcal{X}$ represents a feasible subset $q \in \mathcal{Q}$ with $x_{j}=1 \Leftrightarrow$ $e_{j} \in q$. Then, the objective function is defined by

$$
z_{i}(x, c):=\sum_{j=1}^{n} c_{i, j} x_{j} \forall i \in\{1, \ldots, k\} .
$$

Even though there exist several publications applying some robustness criterion to 
multi-objective uncertain combinatorial optimization problems (e.g., Mavrotas et al., 2015; Cintrano et al., 2017), their notions of robustness do not follow the definitions presented in this section, but are mostly based on concepts we rather associate with sensitivity analysis or stochastic optimization.

To the best of our knowledge, apart from the publications constituting the cumulative part of this thesis, only Kuhn et al. (2016) have developed solution approaches for multi-objective uncertain combinatorial problems applying some of the robustness concepts defined above. They confine their work to bi-objective problems with uncertainty in only one of the objective functions.

In the works summarized in Chapter 3 of this thesis (Raith et al., 2018b,a; Schmidt et al., 2018), multi-objective uncertain combinatorial optimization problems with any fixed number of uncertain objectives are considered, with a focus on shortest path problems. The authors develop approaches to find robust efficient solutions with respect to the concepts given in Definitions 2.9-2.12 and 2.14, considering finite, interval and bounded uncertainty sets. 



\section{Summary of the Publications}

The cumulative part of this thesis consists of three research papers, which are summarized in this chapter. The author's own contribution to the respective manuscript is described at the end of each summary.

Section 3.1 summarizes the article Raith et al. (2018b), see Addendum A.1, which is published in the European Journal of Operational Research. The authors introduce two approaches to find pointMR efficient (or setMR efficient) solutions for multiobjective uncertain combinatorial optimization problems with objective-wise bounded uncertainty. From the general solution approaches they develop specific algorithms for the shortest path problem, which they compare experimentally.

The article Raith et al. (2018a), which is summarized in Section 3.2 and included in this thesis in Addendum A.2, is published in the journal Networks. So far, it has not been included in an issue, but the early view version is available online. In this paper, labeling algorithms for finding robust efficient solutions for the shortest path problem with a finite uncertainty set are developed, considering several different concepts of robust efficiency. Their performance is analyzed in an extensive numerical evaluation. Section 3.3 contains a summary of the manuscript Schmidt et al. (2018), see Addendum A.3, which is available as preprint and has been submitted to the European Journal of Operational Research in January 2018. The authors introduce two scalarization methods for finding pointMR efficient or setMR efficient solutions for multi-objective uncertain optimization problems. They examine how the scalarized problems may be approached for combinatorial problems with particular uncertainty sets.

\subsection{Multi-Objective Minmax Robust Combinatorial Optimization with Cardinality-Constrained Uncertainty}

In Raith et al. (2018b), which we refer to as Publication 1, the authors consider multiobjective uncertain combinatorial problems with objective-wise bounded uncertainty, which they call cardinality-constrained uncertainty. They develop two approaches to find pointMR efficient (hence also setMR efficient) solutions: First they extend an algorithm for the single-objective minmax robust problem with bounded uncertainty to the multi-objective case with objective-wise bounded uncertainty. In addition, they 
provide an enhancement of the algorithm for one objective as well as a new proof of its validity, which they extend to prove the functionality of the multi-objective version. In the second approach, they transfer the multi-objective uncertain combinatorial optimization problem into a multi-objective deterministic optimization problem, whose efficient solutions form a superset of the robust efficient solutions for the original problem. They apply this approach to the shortest path problem by adjusting a labeling algorithm. Both algorithms are tested on a shortest path problem occurring in hazardous material transportation.

Note that the notation in Publication 1 differs slightly from the notation used in this thesis, e.g., the authors use $z^{\mathrm{R}}(q)$ instead of $\bar{z}(q)$ (see Definition 2.11) and $c_{i}\left(e_{j}\right)$ instead of $c_{i, j}$. In this summary, we use the notation introduced in Chapter 2.

\section{Deterministic Subproblems Algorithm (DSA) for Single-Objective Problems}

Bertsimas and Sim (2003) show that a minmax robust optimal solution for a singleobjective uncertain combinatorial problem with bounded uncertainty can be found by solving $n+1$ deterministic problems, which we call deterministic subproblems. They assume that the elements in $E$ and hence the indices of $\hat{c}, \delta$ are sorted with respect to the interval lengths, i.e., such that $\delta_{1} \geqq \delta_{2} \geqq \ldots \geqq \delta_{n} \geqq \delta_{n+1}:=0$. They define for every $l \in\{1, \ldots, n+1\}$ the problem

$$
\mathcal{P}^{\prime}(l) \quad \min _{q \in \mathcal{Q}} g^{l}(q) \quad \text { with } g^{l}(q):=\sum_{e_{j} \in q} \hat{c}_{j}+\Gamma \cdot \delta_{l}+\sum_{\substack{e_{j} \in q, j \leqq l}}\left(\delta_{j}-\delta_{l}\right) .
$$

Since the summand $\Gamma \cdot \delta_{l}$ is solution-independent, every deterministic subproblem $\mathcal{P}^{\prime}(l)$ can be interpreted as a combinatorial problem of the same type as the underlying problem with costs

$$
c_{j}^{l}:= \begin{cases}\hat{c}_{j}+\left(\delta_{j}-\delta_{l}\right) & \text { for } j<l \\ \hat{c}_{j} & \text { else. }\end{cases}
$$

The Deterministic Subproblems Algorithm (DSA) solves $\mathcal{P}^{\prime}(l)$ for $l=1, \ldots, n+1$ and chooses among the obtained solutions the solution with minimal objective value. Hence, in case the underlying deterministic problem is polynomially solvable (e.g., the shortest path problem or the minimum spanning tree problem), the DSA has polynomial runtime.

Bertsimas and Sim (2003) prove with help of dualization that the DSA indeed finds a minmax robust optimal solution. In Publication 1, the authors introduce an alternative proof, which they later extend to prove their algorithm for the multi-objective case: They show that $g^{l}(q) \geqq \max _{c \in \mathcal{U}^{b}} z(q, c) \forall q \in \mathcal{Q}, l \in\{1, \ldots, n+1\}$ and that for each $q \in \mathcal{Q}$ there exists $\tilde{l} \in\{1, \ldots, n+1\}$ such that $g^{\tilde{l}}(q)=\max _{c \in \mathcal{U}^{b}} z(q, c)$. Therefore, every minmax robust optimal solution is optimal for at least one deterministic 
subproblem $\mathcal{P}^{\prime}(\tilde{l})$, whose optimal objective value is smaller than or equal to the optimal objective values of the other subproblems.

According to the results by Bertsimas and Sim (2003); Park and Lee (2007); Lee and Kwon (2014), the number of subproblems to be solved can be reduced to $\left\lceil\frac{n-\Gamma}{2}\right\rceil+1$. The authors of Publication 1 show that, in addition, a subproblem needs not to be solved, if the solution of an already solved subproblem has the following property.

Lemma 3.1 (Publication 1, Lemma 9). Let $1 \leqq \tilde{l}<l \leqq|E|+1$ and let $q^{\tilde{l}}$ be an optimal solution for $\mathcal{P}^{\prime}(\tilde{l})$. If $q^{\tilde{l}}$ does not contain any of the elements $e_{1}, \ldots, e_{l-1}$, then it is optimal for $\mathcal{P}^{\prime}(l)$.

The authors point out that even though this result does not improve the theoretical worst case runtime, their experimental evaluation shows its use for practical applications.

\section{DSA for Multi-Objective Problems}

For the validity of the DSA for single-objective problems it is crucial that the elements in $E$, and hence the indices of $\hat{c}, \delta$, are sorted such that the entries of $\delta$ are decreasing. However, the authors of Publication 1 point out that in the multiobjective case a respective order of the elements does not necessarily exist: if they are sorted such that the interval lengths in the first objective are decreasing, i.e., $\delta_{1,1} \geqq \delta_{1,2} \geqq \ldots \geqq \delta_{1, n}$, the interval lengths in the other objectives are not necessarily decreasing as well. Therefore, given a multi-objective uncertain combinatorial optimization problem with objective-wise bounded uncertainty, deterministic subproblems cannot be defined analogous to the single-objective case. However, the authors define suitable multi-objective deterministic subproblems in a similar way: For each $l=\left(l_{1}, \ldots, l_{k}\right) \in L:=\{1, \ldots, n+1\} \times \ldots \times\{1, \ldots, n+1\}$ they define

$$
\left(\mathcal{M O P}^{\prime}(l)\right) \min _{q \in \mathcal{Q}} g^{l}(q) \quad \text { with } g^{l}(q):=\left(\begin{array}{c}
\sum_{e_{j} \in q} \hat{c}_{1, j}+\Gamma_{1} \cdot \bar{\delta}_{l_{1}}^{1}+\sum_{e_{j} \in q \cap E_{l_{1}}^{1}}\left(\delta_{1, j}-\bar{\delta}_{l_{1}}^{1}\right) \\
\vdots \\
\sum_{e_{j} \in q} \hat{c}_{k, j}+\Gamma_{k} \cdot \bar{\delta}_{l_{k}}^{k}+\sum_{e_{j} \in q \cap E_{l_{k}}^{k}}\left(\delta_{k, j}-\bar{\delta}_{l_{k}}^{k}\right)
\end{array}\right),
$$

where, for every $i \in\{1, \ldots, k\}$ and $l_{i} \in\{1, \ldots, n\}, E_{l_{i}}^{i} \subseteq E$ contains a set of $l_{i}$ elements with largest interval lengths w.r.t. the $i$-th objective, i.e., $\left|E_{l_{i}}^{i}\right|=l_{i}$ and

$$
\delta_{i, j} \geqq \delta_{i, j^{\prime}} \forall e_{j} \in E_{l_{i}}^{i}, e_{j^{\prime}} \notin E_{l_{i}}^{i} .
$$

Further, for all $i \in\{1, \ldots, k\}$, they define $E_{n+1}^{i}:=E, \bar{\delta}_{n+1}^{i}:=0$ and

$$
\bar{\delta}_{l_{i}}^{i}:=\min _{e_{j} \in E_{l_{i}}^{i}} \delta_{i, j} \forall l_{i} \in\{1, \ldots, n\},
$$


hence, $\bar{\delta}_{l_{i}}^{i}$ equals the $l_{i}$-largest of the interval lengths w.r.t. the $i$-th objective. Note that $E_{l_{i}}^{i}$ and $\bar{\delta}_{l_{i}}^{i}$ are not variables, but can be precomputed.

Here, the efficient solution of the subproblems can be found by solving a multiobjective deterministic combinatorial problem of the same type as the underlying problem with costs

$$
c_{i, j}^{l}:= \begin{cases}\hat{c}_{i, j}+\left(\delta_{i, j}-\bar{\delta}_{l_{i}}^{i}\right) & \text { for } e_{j} \in E_{l_{i}}^{i} \\ \hat{c}_{i, j} & \text { else. }\end{cases}
$$

The authors propose an algorithm (Algorithm 3 in Publication 1), referred to as DSA: First, it searches a complete set $O P T^{l}$ of efficient solutions for $\mathcal{M O P}^{\prime}(l)$ for every $l \in L$. It then returns all $q \in \bigcup_{l \in L} O P T^{l}$ for which there exists no $q^{\prime} \in \bigcup_{l \in L} O P T^{l}$ with $\bar{z}\left(q^{\prime}\right) \leq \bar{z}(q)$. The authors prove that the DSA indeed finds a complete set of efficient solutions for $\min _{q \in \mathcal{Q}} \bar{z}(q)$ (Publication 1, Theorem 10), because $g^{l}(q) \geqq \bar{z}(q)$ for all $q \in \mathcal{Q}, l \in L$ and for each $q \in \mathcal{Q}$ there exists $\tilde{l} \in L$ such that $\bar{z}(q)=g^{\tilde{l}}(q)$. Note that the found solutions are both pointMR efficient and setMR efficient, because the problem is objective-wise uncertain (see Definition 2.11 and Lemma 2.13).

The authors show that the number of subproblems to be solved can be reduced to $\prod_{i=1}^{k}\left(\left\lceil\frac{|E|-\Gamma_{i}}{2}\right\rceil+1\right)$ (Publication 1, Lemma 12), using the results for the singleobjective problem by Bertsimas and Sim (2003); Park and Lee (2007); Lee and Kwon (2014). Furthermore, a result similar to Lemma 3.1 can be used to skip some of these subproblems, if the solutions of a formerly solved subproblem fulfill a special condition (Publication 1, Lemma 13). We refer to this method as solution checking. In addition, the authors show that the number of subproblems to be solved can further be reduced significantly, if the problem has [partly/.] objective-independent element order, i.e., if the elements can be ordered such that the interval lengths are in decreasing order for [several/all] objectives and the respective $\Gamma_{i}$ are identical (Publication 1, Lemma 17). In case of objective-independent element order, $\left\lceil\frac{|E|-\Gamma_{i_{1}}}{2}\right\rceil+1$ subproblems suffice.

\section{Bottleneck Approach}

The authors present a second solution approach, where the multi-objective uncertain problem is transformed to a multi-objective deterministic problem, whose set of efficient solutions contains a complete set of efficient solutions for $\min _{q \in \mathcal{Q}} \bar{z}(q)$.

For this, they use the following notation for the $h$-greatest interval length in a solution $q \in \mathcal{Q}$ w.r.t. a given $i \in\{1, \ldots, k\}$ (see Publication 1, Definition 18): for a subset $q \subseteq E$ and given interval lengths $\delta_{i, j}$ for all $e_{j} \in E$, they sort the elements in $q$ by decreasing interval lengths and define $h$ - $\max _{e_{j} \in q} \delta_{i, j}$ as the interval length of the $h$-th element according to this sorting.

They first explain their approach for single-objective problems and then extend it 
to the multi-objective case. For a given MOUCO with $k$ objectives they define a multi-objective deterministic problem with $\sum_{i=1}^{k}\left(\Gamma_{i}+1\right)$ objectives:

$$
\operatorname{MODCO} \min _{q \in \mathcal{Q}} z^{\mathbf{D}}(q) \quad \text { with } z^{\mathbf{D}}(q):=\left(\begin{array}{c}
\sum_{e_{j} \in q} \hat{c}_{1, j} \\
\max _{e_{j} \in q} \delta_{1, j} \\
2-\max _{e_{j} \in q} \delta_{1, j} \\
\vdots \\
\Gamma_{1-\max _{e_{j} \in q} \delta_{1, j}} \\
\sum_{e_{j} \in q} \hat{c}_{2, j} \\
\max _{e_{j} \in q} \delta_{2, j} \\
\vdots \\
\Gamma_{k-\max _{e_{j} \in q} \delta_{k, j}}
\end{array}\right) .
$$

They show that every pointMR efficient solution for MOUCO is an efficient solution for MODCO and that a complete set of efficient solutions for MODCO contains a complete set of efficient solutions for $\min _{q \in \mathcal{Q}} \bar{z}(q)$ (Publication 1, Theorem 22).

\section{Label Setting Algorithm (LSA) for the Multi-Objective Uncertain Shortest Path Problem}

To use the bottleneck approach, one needs an algorithm to find a complete set of efficient solutions for MODCO. In Publication 1, the authors introduce such an algorithm for the multi-objective uncertain shortest path problem with non-negative edge lengths, where $E$ is the edge set of a graph and $\mathcal{Q}$ is the set of all simple paths from a start node $s$ to a termination node $t$. They adjust the label setting algorithm of Martins (1984) for the multi-objective deterministic shortest path problem. The structure of the algorithm is the same as that of the algorithm of Martins: A label at a node $v$ represents a path $q$ from $s$ to $v$. It has a cost vector $y(l)$, equal to the cost of $q$, and a predecessor label $l^{\prime}$ at the predecessor node $v^{\prime}$ of $v$ on $q$, representing the subpath of $q$ from $s$ to $v^{\prime}$. Starting with a temporary label of cost 0 at $s$, as long as there exists at least one temporary label, the algorithm

1. chooses a temporary label $l^{\prime}$ at a node $v^{\prime}$ to make it permanent instead of temporary,

2. produces new temporary labels at the end of the outgoing edges of $v^{\prime}$, whose predecessor label is $l^{\prime}$,

3. deletes every temporary label $l$ for which a label $\tilde{l}$ at the same node with $y(\tilde{l}) \leq y(l)$ exists.

For the classical multi-objective shortest path problem, where each objective is the sum of the edge costs w.r.t. this objective, the cost $y(l)$ of a new label is obtained by 
adding the cost of the predecessor label $y\left(l^{\prime}\right)$ to the cost of the last edge $e_{j}:=\left(v^{\prime}, v\right)$. In Publication 1, the authors define a new procedure in order to obtain suitable label costs for MODCO: They add the nominal costs of $e_{j}$ to the components of $y(l)$ corresponding to the sum objectives in MODCO. The interval lengths $\delta_{i, j}$ associated to $e_{j}$ are compared to the other components of $y(l)$ and inserted at the right place (Publication 1, Algorithm 6).

In Step 1, the algorithm in Publication 1 chooses the label with the smallest aggregated costs, as proposed by Iori et al. (2010). It also differs from the Algorithm of Martins in Step 3: if several labels with the same costs at the same node exist, all but one of them are deleted, because the aim is to find a complete set of efficient solutions, not all efficient solutions.

The authors show that the adjusted labeling algorithm indeed finds a complete set of efficient solutions for MODCO (Publication 1, Theorem 27) and propose an additional filtering step to obtain a complete set of efficient solutions for $\min _{q \in \mathcal{Q}} \bar{z}(q)$ (see Publication 1, Algorithm 7 and Corollary 28). In the following the entire algorithm, including the filtering step, is called $L S A$.

\section{Experimental Evaluation}

The authors compare the performance of the two algorithms DSA and LSA for a multi-objective uncertain shortest path problem arising from hazardous material transportation. The aim is to find a path in a road network that minimizes travel time on the one hand and the population affected by the hazardous material in a potential accident on the other hand. Both objectives are uncertain, because the travel time depends, for example, on traffic congestion and the population in the area is influenced, e.g., by local events or regular shifts in population during the work day. The travel time intervals are obtained via an iterative algorithm to solve a traffic assignment problem. The population interval lengths are chosen randomly up to a given percentage of the assigned nominal values. Varying this percentage, referred to as population uncertainty, several different instances are constructed.

All methods to reduce the number of subproblems of the DSA, which are described above, are implemented. The subproblems of the DSA are solved with an implementation of the algorithm of Martins (1984) with the same adjustments in Steps 1 and 3 as in the LSA: in Step 1 the temporary label with the smallest aggregated cost is chosen and in Step 3, if there exist labels with the same cost at the same node, all but one are deleted.

The results show that the minimal number of robust efficient solutions in a complete set and the runtime of both algorithms generally increases with increasing population uncertainty. Comparing the performance of the two algorithms, the authors observe that with increasing values of $\Gamma_{i}$ the runtime of the DSA decreases, whereas the runtime of the LSA increases (see Figure 3.1). This can be explained by the decreasing number of subproblems of the DSA and the increasing number of objec- 


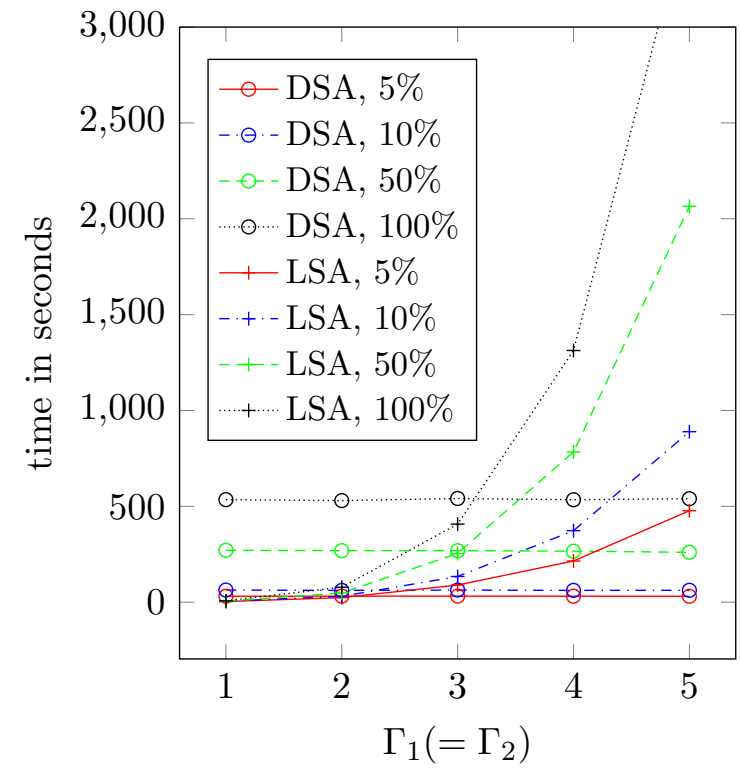

(a) $\Gamma_{1}=\Gamma_{2} \in\{1, \ldots, 5\}$

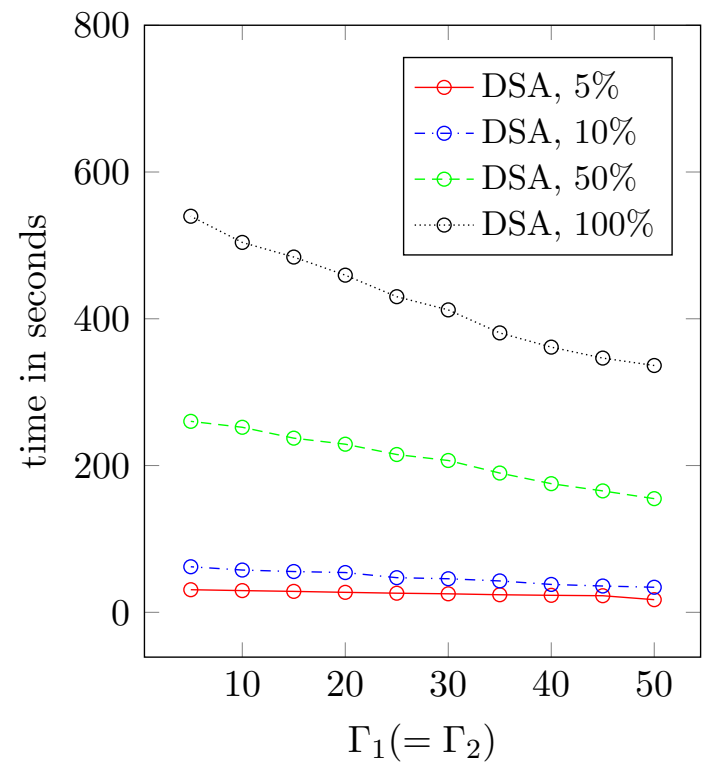

(b) $\Gamma_{1}=\Gamma_{2} \in\{5,10, \ldots, 50\}$

Figure 3.1.: Running time of the DSA and the LSA for several values of $\Gamma_{i}$ and population uncertainty on two different scales (Publication 1, Figure 5).

tives of MODCO, which is solved by the LSA. Indeed, for very small values of $\Gamma_{i}$ the LSA solves the given instances faster than the DSA, whereas the DSA has a better performance for higher values of $\Gamma_{i}$. This is also true if the problem has three objectives instead of two objectives, which is tested on instances with a third (artificial) objective.

The authors further generate an instance with two strongly correlated objective functions, using the travel time as one objective and constructing a second objective by multiplying the nominal times and the interval lengths each by a random factor between 0.9 and 1.1. Both algorithms benefit from the correlation in terms of runtime, but the LSA benefits more: while for $\Gamma_{1}=\Gamma_{2}=4$ the DSA already performed better for all tested instances with two uncorrelated objective functions, the LSA solved the correlated instances faster than the DSA up to $\Gamma_{1}=\Gamma_{2}=26$.

In addition to the comparisons of the DSA and the LSA, the authors investigate the effect of the proposed enhancements: First, they compare the performance of the DSA with solution checking to a version without solution checking. The results show that the algorithm is accelerated substantially if subproblems can be skipped in this way, and it is not significantly slowed down by the procedure even if no subproblems can be skipped. Second, they test the DSA on an instance with objective-independent element order. They compare the performance of the DSA for general instances to a special version $D S A-o i$, which takes into account that the number of subproblems can be reduced further in case of objective-independent element order (Publication 1, 
Lemma 17). As expected, the DSA-oi solves this instance much faster than the general version of the DSA. They also implement a procedure to check whether an instance has objective-independent element order, which does not take much time in comparison to the total running time of the DSA.

The authors conclude that the DSA solves most of the tested instances faster, but that the LSA performs better for small values of $\Gamma_{i}$, in particular if the objectives are strongly correlated. When implementing the DSA, they recommend to use the proposed enhancements and to check whether the special version for instances with (partial) objective-independent element order can be used, because the additional procedures do not take much time in comparison to the total running time and, if subproblems can be skipped, the algorithm is accelerated significantly.

\section{Own Contribution}

This article is joint work with Andrea Raith, Marie Schmidt and Anita Schöbel. The ideas leading to this publication were developed cooperatively by all four authors. Most of the details, including the algorithms, the technicalities in the proofs and the examples, were contributed by myself, of course with consultation of the other authors. I have done approximately half of the implementations and the main part of the experiments. Most of the text and figures, both in the theoretical and the experimental part, were produced by myself.

\subsection{Extensions of Labeling Algorithms for Multi-Objective Uncertain Shortest Path Problems}

This section summarizes the article Raith et al. (2018a), which we refer to as Publication 2. In this paper, the authors consider the multi-objective uncertain shortest path problem with finite uncertainty. They aim to find multi-scenario efficient, flimsily, highly, point-based minmax and set-based minmax robust efficient solutions. First, they analyze why it is, for most of the considered concepts, not straightforward to use labeling algorithms for the multi-objective uncertain problem. They then develop algorithms to find robust efficient solutions, by either extending a generic multiobjective label correcting algorithm or using it repeatedly. In a numerical study, the authors analyze and compare the performance of the developed algorithms on two different types of networks.

An instance of the multi-objective uncertain shortest path problem (MOUSP) is given by a graph $G=(V, E)$ with node set $V$ and edge set $E$, a start node $s \in V$, an end node $t \in V$ and an uncertainty set $\mathcal{U} \subseteq \mathbb{R}^{k \times n}$, containing all possible edge costs. 
For every $v \in V$, let $\mathcal{Q}^{v}$ denote the set of all simple paths from $s$ to $v$. MOUSP is then a special case of MOUCO with element set $E$, feasible set $\mathcal{Q}^{t}$ and uncertainty set $\mathcal{U}$ (see page 12 ). In this publication, finite uncertainty sets are considered, i.e., $\mathcal{U}=\left\{c^{1}, c^{2}, \ldots, c^{r}\right\}$ for some $r \in \mathbb{N}$.

To keep a consistent notation throughout the thesis, the notation in this summary differs from the notation in the article itself. At some places we point out the original notation, to allow an easier understanding when looking something up in the article. For example, in the notation of Publication 2, the uncertainty set $\mathcal{U}$ is given as a set of scenarios $\left\{\xi_{1}, \ldots, \xi_{r}\right\}$ and the costs as a function $c: \mathcal{Q} \times \mathcal{U} \rightarrow \mathbb{R}^{k}$, where $c_{i}\left(e_{j}, \xi_{d}\right)$ is identical to $c_{i, j}^{d}$ in our notation. Hence, an instance of MOUSP is given as $(G, \mathcal{U}, c, s, t)$ in Publication 2 instead of $(G, \mathcal{U}, s, t)$ in our notation.

\section{General Label Correcting Algorithm}

The authors consider a generic multi-objective label correcting algorithm with label selection method (see, e.g., Guerriero and Musmanno, 2001), called Algorithm 1. A label at a node $v$ represents a path $q$ from $s$ to $v$. It has a cost $z(l)$, which equals the cost of $q$, and a predecessor label $l^{\prime}$ at the predecessor node $v^{\prime}$ of $v$ on $q$, representing the subpath of $q$ from $s$ to $v^{\prime}$.

The label correcting algorithm starts with an empty label set $\mathcal{L}$ and a second label set $\mathcal{T}$ containing a label of cost 0 at node $s$. As long as $\mathcal{T}$ is not empty, the algorithm

1. chooses a label $l^{\prime}$ in $\mathcal{T}$ at a node $v^{\prime}$ and moves it to the label set $\mathcal{L}$ instead,

2. produces new labels at the end of the outgoing edges of $v^{\prime}$, whose predecessor label is $l^{\prime}$,

3. adds every new label $l$ to $\mathcal{T}$, if there exists no label $\tilde{l} \in \mathcal{T} \cup \mathcal{L}$ at the same node that has identical cost or dominates $l$,

4. deletes every label $\tilde{l} \in \mathcal{T} \cup \mathcal{L}$ that is dominated by a new label $l \in \mathcal{T}$ at the same node.

Afterwards, it returns all labels in $\mathcal{L}$ at $t$.

In the multi-objective deterministic case, the cost of a path $q$, i.e., the cost of the label $l$ representing $q$, is the sum of the cost vectors of the edges in $q$. To compute $z(l)$, one adds the cost of its predecessor label to the cost of the last edge in $q$. A label $l$ dominates another label $\tilde{l}$, if $z(l) \leq z(\tilde{l})$. When the algorithm stops, the labels at $t$ represent a complete set of efficient paths from $s$ to $t$.

The authors point out that in the uncertain case with finite uncertainty set, the cost 
of an edge $e_{j}$ can be written as a matrix

$$
Z\left(e_{j}\right):=\left(\begin{array}{cccc}
c_{1, j}^{1} & c_{1, j}^{2} & \ldots & c_{1, j}^{r} \\
c_{2, j}^{1} & c_{2, j}^{2} & \ldots & c_{2, j}^{r} \\
\vdots & \vdots & \ddots & \vdots \\
c_{k, j}^{1} & c_{k, j}^{2} & \ldots & c_{k, j}^{r}
\end{array}\right) \in \mathbb{R}^{k \times r}
$$

the cost of a path as $Z(q):=\sum_{e \in q} Z(e)$ and the cost of a label $l$ representing $q$ as $Z(l):=Z(q)$. (In the original paper, $[Z(e) / Z(q) / Z(l)]$ is denoted as $[c(e) / z(q) / z(l)]$.) For a given concept of robust efficiency, they define a complete set of robust efficient paths as a set of robust efficient paths $\mathcal{Q}^{\prime} \subseteq \mathcal{Q}^{t}$, such that for each robust efficient path $q$ there exists $q^{\prime} \in \mathcal{Q}^{\prime}$ with $Z(q)=Z\left(q^{\prime}\right)$.

Using cost matrices instead of cost vectors, the label setting algorithm can easily be transferred to the uncertain case, if a suitable definition of dominance is given. The authors show that a complete set of robust efficient solutions can be found with a straightforward transfer of Algorithm 1, referred to as Algorithm 1', if the concept of robust efficiency fulfills the following two conditions (see Publication 2, Theorem 8).

1. Principle of optimality: For every instance $(G, \mathcal{U}, s, t)$ of MOUSP we require: if $q \in \mathcal{Q}^{t}$ is a robust efficient path for $(G, \mathcal{U}, s, t)$, then for every node $v$ in $q$ its subpath $q_{s, v}$ from $s$ to $v$ is robust efficient for the instance $(G, \mathcal{U}, s, v)$.

2. For every $k, r \in \mathbb{N}$ there exists a binary (dominance) relation $R \subseteq \mathbb{R}^{k \times r} \times \mathbb{R}^{k \times r}$ with the following properties:

a) The relation is consistent with the concept of robust efficiency: for all instances with $k$ objectives and $|\mathcal{U}|=r$ :

$$
q \in Q^{t} \text { is robust efficient } \Leftrightarrow \nexists q^{\prime} \in Q^{t}:\left(Z\left(q^{\prime}\right), Z(q)\right) \in R
$$

b) Domination property: For all instances with $k$ objectives and $|\mathcal{U}|=r$ :

$$
\begin{aligned}
& q \in Q^{t} \text { is not robust efficient } \\
\Rightarrow & \exists \text { robust efficient } q^{\prime} \in Q^{t}:\left(Z\left(q^{\prime}\right), Z(q)\right) \in R
\end{aligned}
$$

c) $R$ is transitive, i.e., $\left(Y^{1}, Y^{2}\right) \in R,\left(Y^{2}, Y^{3}\right) \in R \Rightarrow\left(Y^{1}, Y^{3}\right) \in R$.

We say that $q^{\prime}$ dominates $q$ if $\left(Z\left(q^{\prime}\right), Z(q)\right) \in R$.

Condition 2 defines the notion of dominance used in Steps 3 and 4 . Further, the instance needs to be conservative, i.e., the cost of every circle $C$ in $G$ is either 0 or we have $(Y, Y+Z(C)) \in R$ for all $Y \in \mathbb{R}^{k \times r}$. 


\section{Labeling for the Multi-Objective Robust Shortest Path Problem}

The authors investigate whether the considered concepts (multi-scenario efficiency, flimsily and highly robust efficiency, pointMR and setMR efficiency) fulfill the two conditions given above. In case any of the conditions is not fulfilled, they propose algorithms to nevertheless find a complete set of robust efficient solutions. These algorithms are either an extension of Algorithm 1' (extended labeling algorithms) or solve Algorithm 1' several times for auxiliary problems with different definitions of dominance and compute the solution set from the obtained solutions for the auxiliary problems (repeated labeling algorithms).

For multi-scenario efficiency with finite uncertainty set, MOUSP reduces to a multiobjective deterministic problem (see Definition 2.14). Hence, one can directly use the label correcting algorithm for multi-objective deterministic problems, Algorithm 1.

For flimsily robust efficiency (see Definition 2.10), the authors show that the principle of optimality (Condition 1) is fulfilled, but that no dominance relation exists with Property $2 \mathrm{a}$ as required in Condition 2.

They introduce an extension of Algorithm 1' to nevertheless find a complete set of flimsily robust efficient solutions (EL-Flimsily), see Algorithm 2 in Publication 2. For each label $l$, an additional vector $x(l) \in\{0,1\}^{r}$ is stored, which is set to 0 in the beginning of the algorithm. When comparing two labels $l, \tilde{l}$ in Step 3 or Step 4, one compares them for each scenario independently, i.e., the cost matrices are compared column-wise. If $Z(l)_{(\cdot, d)} \leq Z(\tilde{l})_{(\cdot, d)}$, then the entry $x_{d}(\tilde{l})$ is set to 1 , indicating that the label $\tilde{l}$ is dominated in scenario $c^{d}$. A label is deleted if it is dominated in every scenario. The authors show that EL-Flimsily indeed finds a complete set of flimsily robust efficient solutions, if the instance fulfills a condition similar to conservativeness (Publication 2, Theorem 12).

The authors also propose a repeated labeling algorithm (RL-Flimsily), see Algorithm 3 in Publication 2. For each $c^{d} \in \mathcal{U}$ it executes Algorithm 1' with the following definition of dominance: a label $l$ dominates another label $\tilde{l}$ at the same node, if $Z(l)_{(\cdot, d)} \leq Z(\tilde{l})_{(\cdot, d)}$. Afterwards, the union of the obtained solution sets is returned.

The authors show further that for highly robust efficiency (see Definition 2.9), Condition 1 is fulfilled, too, but no dominance relation exists with Property $2 \mathrm{~b}$ as required in Condition 2. Since the highly robust efficient solutions are exactly the flimsily robust efficient solutions that are not dominated in any scenario, the authors propose an algorithm (EL-Highly) that executes EL-Flimsily and returns only those labels $l$ with $x(l)=0$ (Publication 2, Algorithm 4). As an alternative they propose a repeated labeling algorithm (RL-Highly), which works similar to RL-Flimsily, but returns the intersection of the obtained solution sets instead of their union (Publication 2, Algorithm 5). 
Finally, the authors show that suitable dominance relations exist for pointMR and setMR efficiency. For pointMR efficiency that is $R^{\text {point }}$ defined as

$$
\left(Y, Y^{\prime}\right) \in R^{\text {point }} \Leftrightarrow\left(\begin{array}{c}
\max _{d=1, \ldots, r} Y_{1, d} \\
\vdots \\
\max _{d=1, \ldots, r} Y_{k, d}
\end{array}\right) \leq\left(\begin{array}{c}
\max _{d=1, \ldots, r} Y_{1, d}^{\prime} \\
\vdots \\
\max _{d=1, \ldots, r} Y_{k, d}^{\prime}
\end{array}\right)
$$

and for setMR efficiency that is $R^{\text {set }}$ given by

$$
\left(Y, Y^{\prime}\right) \in R^{\mathrm{set}} \Leftrightarrow \bigcup_{d=1, \ldots, r}\left\{Y_{(\cdot, d)}\right\} \subseteq \bigcup_{d=1, \ldots, r}\left\{Y_{(\cdot, d)}^{\prime}\right\}-\mathbb{R}_{\geq}^{k}
$$

On the other hand, both concepts do not fulfill Condition 1, i.e., subpaths of robust efficient paths are not necessarily robust efficient themselves.

To overcome this obstacle, the authors adopt an idea introduced by $\mathrm{Yu}$ and Yang (1998); Kouvelis and Yu (1997) for the single-objective case with integer edge costs, and introduce an extended labeling algorithm to find pointMR and setMR efficient solutions (Publication 2, Algorithm 6).

Assuming integer edge costs, each label $l$ at a node $v$ is assigned a prediction matrix $A(l) \in \mathbb{Z}^{k \times r}$ (denoted $a(l)$ in Publication 2), which contains assumed costs for continuing the represented path from $v$ to $t$. Hence, every path from $s$ to $v$ can be represented by several labels with different prediction matrices.

The algorithm starts with several labels of cost 0 at $s$, one for every prediction matrix with integer entries that lie between precomputed lower and upper bounds. When generating a new label, its prediction matrix is obtained by subtracting the last edge's cost from the prediction matrix of its predecessor label. In steps 3 and 4 only those labels are compared, whose prediction matrices are identical. A label $l$ is deleted, if the predicted cost $Z(l)+A(l)$ of the whole path from $s$ to $t$ is dominated by the predicted $\operatorname{cost} Z(\tilde{l})+A(\tilde{l})$ of another label $\tilde{l}$ at the same node with the same prediction matrix. Using the dominance relation $\left[R^{\text {point }} / R^{\text {set }}\right]$, the resulting algorithm [EL-PB/EL-SB] finds a complete set of [pointMR/setMR] efficient solutions for conservative instances (Publication 2, Theorem 16).

The results of this section are summarized in Table 3.1. All presented algorithms run in pseudo-polynomial time for integer edge costs, if the number of objectives and scenarios is fixed.

\section{Experiments}

The authors test their algorithms on two types of networks. Grid networks contain a set of nodes that can be interpreted as a two-dimensional grid where vertically or horizontally neighbored nodes are connected by edges. An additional [start/termination] 


\begin{tabular}{|l|lll|}
\hline Concept of robust efficiency & Cond. 1 & Cond. 2 & Algorithms \\
\hline multi-scenario efficiency & yes & yes & Algorithm 1 \\
flimsily robust efficiency & yes & no & EL-Flimsily, RL-Flimsily \\
highly robust efficiency & yes & no & EL-Highly, RL-Highly \\
pointMR efficiency & no & yes & EL-PB \\
setMR efficiency & no & yes & EL-SB \\
\hline
\end{tabular}

Table 3.1.: Summary of which conditions are satisfied for which concept of robust efficiency and which algorithms can be used to find a complete set of robust efficient solutions (Publication 2, Table 1).

node is connected to all nodes in the [first/last] column of the grid. In NetMaker networks the nodes are enumerated such that $s$ is the first and $t$ the last node. Most edges connect nodes whose indices do not differ more than a given threshold. This prevents paths from $s$ to $t$ with very few edges, which would then easily dominate all other paths.

To obtain so called random instances, the integer edge costs are generated randomly from given intervals, for details see Addendum A.2, pages 83-84. To obtain instances with correlated scenarios (called correlated instances), only the cost matrices for the first scenario are generated in this way and the costs for all other scenarios are chosen similar to the first scenario.

The main focus of the experimental evaluation is to compare the extended and the repeated labeling algorithms. Therefore, the authors mainly investigate the performance of the algorithms EL-Flimsily and RL-Flimsily (as the runtimes of the corresponding algorithms for finding highly robust efficient solutions are similar). We summarize the main results.

- The repeated labeling algorithm RL-Flimsily solves most instances faster than the extended labeling algorithm EL-Flimsily. This can be explained by the high number of labels created and kept at each node in EL-Flimsily, because a label is only deleted when it has been detected to be dominated in every scenario.

- The runtime of both algorithms mostly increases with the number of objectives, the number of scenarios and the size of the network. In case of grid networks, the width plays a more important role than the height.

- The performance of RL-Flimsily is similar for random and correlated instances, whereas EL-Flimsily solves correlated instances much faster than random instances. An explanation for this is that, for correlated scenarios, a label dominating another in one scenario tends to dominate it in all other scenarios as well, hence for discarding a label in EL-Flimsily, often one dominating label 
suffices. RL-Flimsily, on the other hand, executes Algorithm 1' once for each scenario, even if the costs are identical for all scenarios. For some correlated instances, in particular for NetMaker instances with higher numbers of objectives and scenarios, EL-Flimsily is faster than RL-Flimsily.

- For grid networks, all edge cost components for an instance were either chosen from $\{1, \ldots, 10\}$ or from $\{1, \ldots, 100\}$. For random instances, the runtime of both algorithms is higher if the wider range is used. For correlated scenarios, however, EL-Flimsily runs faster on instances with the wider cost range, whereas RL-Flimsily runs slower.

- The authors also investigate the number of solutions. Increasing the number of objectives or the network size leads to an increasing number of flimsily and highly robust efficient solutions. For grid networks, the number of flimsily robust efficient solutions increases more with increasing width than with increasing height, which explains the different influence of the grid's width and height on the runtime of the algorithms. When the number of scenarios increases, the number of flimsily robust efficient solutions tends to increase, whereas the number of highly robust efficient solutions tends to decrease. Correlated instances have more highly robust efficient solutions than random instances.

In addition, the authors investigate the performance of EL-PB and EL-SB on small grid network instances. Besides the network size and the number of objectives and scenarios, the values of the edge cost components strongly influence the performance of both algorithms as well: the runtime increases significantly for an increasing number of prediction matrices, which is determined by the edge cost components. The runtime of EL-PB is lower and increases more slowly than that of EL-SB, because the dominance check in EL-PB takes less time. In comparison, the algorithms for finding flimsily and highly robust efficient solutions are much faster and can be used for considerably bigger instances.

\section{Own Contribution}

This paper is joint work with A. Raith, M. Schmidt and A. Schöbel. Even though the ideas were developed and discussed among all authors, most of the ideas for the algorithms were introduced by myself. Also, the major part of the details, including algorithms and examples, were my contribution. I have written most of the theoretical chapter and a smaller part of the experimental evaluation. The main part of the implementations was done by myself and a student I supervised. I have done part of the experiments and their analysis, though the major part of the experiments was conducted and evaluated by A. Raith. 


\subsection{Min-Ordering and Max-Ordering Scalarization Methods for Multi-Objective Robust Optimization}

In Schmidt et al. (2018), referred to as Publication 3, two scalarization methods for multi-objective uncertain optimization problems are developed: the min-ordering and the max-ordering method. The authors show that all pointMR weakly efficient solutions can be found with the max-ordering method. The min-ordering method finds setMR weakly efficient solutions, some of which cannot be found with previously known scalarization methods. The authors investigate how to approach the resulting scalarized problems for combinatorial optimization problems with particular uncertainty sets. In case of interval uncertainty they show that the uncertainty set can be reduced to one scenario. They introduce two versions of bounded uncertainty and develop compact mixed integer linear programming (MILP) formulations for the scalarized problems with these uncertainty sets.

\section{Min-Ordering and Max-Ordering Scalarization Methods for Multi-Objective Uncertain Problems}

Given a multi-objective uncertain optimization problem (MOUP) as in Definition 2.7, a weight vector $\lambda \in \mathbb{R}_{>}^{k}$ and a reference point $r \in \mathbb{R}^{k}$, the authors of Publication 3 define the corresponding min-ordering optimization problem as

$$
\mathrm{P}-\min (r, \lambda) \min _{x \in \mathcal{X}} \alpha^{\min }(x, r, \lambda) \quad \text { with } \alpha^{\min }(x, r, \lambda):=\max _{\xi \in \mathcal{U}} \min _{i \in\{1, \ldots, k\}} \lambda_{i}\left(z_{i}(x, \xi)-r_{i}\right)
$$

and the corresponding max-ordering optimization problem as

$\mathrm{P}-\max (r, \lambda) \min _{x \in \mathcal{X}} \alpha^{\max }(x, r, \lambda) \quad$ with $\alpha^{\max }(x, r, \lambda):=\max _{\xi \in \mathcal{U}} \max _{i \in\{1, \ldots, k\}} \lambda_{i}\left(z_{i}(x, \xi)-r_{i}\right)$.

The min-ordering (resp. max-ordering) scalarization method is obtained, similar to other scalarization methods, by varying the parameters $r$ and $\lambda$ and solving the resulting problems $\mathrm{P}-\min (r, \lambda)(\operatorname{resp} . \mathrm{P}-\max (r, \lambda))$.

The authors remark that the max-ordering scalarization method is similar to the weighted Chebyshev method (Ide, 2014; Hassanzadeh et al., 2013), but with arbitrary reference point. They further remark that for $|\mathcal{U}|=1$, the min-ordering scalarization problem can be solved by solving $k$ single-objective deterministic problems, whereas the max-ordering problem is then equivalent to a single-objective minmax robust optimization problem with $k$ scenarios. 


\section{Geometric Characterization}

The authors provide a geometric interpretation of the solutions for $\mathrm{P}-\max (r, \lambda)$ and $\mathrm{P}-\min (r, \lambda)$. With $g(r, \lambda)$ being the line

$$
g(r, \lambda):=\left\{r+\alpha\left(\frac{1}{\lambda_{1}}, \ldots, \frac{1}{\lambda_{k}}\right)^{T}: \alpha \in \mathbb{R}\right\},
$$

and $\partial M$ denoting the boundary of a set $M \subseteq \mathbb{R}^{k}$, they give the following characterization.

Theorem 3.2 (Publication 3, Theorem 10). Let $r \in \mathbb{R}^{k}, \lambda \in \mathbb{R}_{>}^{k}$ be given. A feasible solution $x^{*} \in \mathcal{X}$ is optimal for $P-\max (r, \lambda)$ if and only if there exists $y^{*} \in \mathbb{R}^{k}$ such that $\left(x^{*}, y^{*}\right)$ is an efficient solution for

$$
\begin{aligned}
G-\max (r, \lambda) \quad \min & \\
\text { s.t. } y & \in g(r, \lambda) \cap \partial\left(\bar{z}(x)+\mathbb{R}_{\geqq}^{k}\right) \\
x & \in \mathcal{X} .
\end{aligned}
$$

A feasible solution $x^{*} \in \mathcal{X}$ is optimal for $P$-min $(r, \lambda)$ if and only if there exists $y^{*} \in \mathbb{R}^{k}$ such that $\left(x^{*}, y^{*}\right)$ is an efficient solution for

$$
\begin{aligned}
G-\min (r, \lambda) \quad \min & y \\
\text { s.t. } & y \in g(r, \lambda) \cap \partial\left(z_{\mathcal{U}}(x)-\mathbb{R}_{\geqq}^{k}\right) \\
& x \in \mathcal{X} .
\end{aligned}
$$

This means that the optimal solutions for $\mathrm{P}-\max (r, \lambda)$ can be identified by comparing the intersection points of $g(r, \lambda)$ with $\partial\left(\bar{z}(x)+\mathbb{R}_{\geq}^{k}\right)$ for all $x \in \mathcal{X}$. Similarly, the optimal solutions of $\mathrm{P}-\min (r, \lambda)$ can be identified by comparing the intersection points of $g(r, \lambda)$ with $\partial\left(z_{\mathcal{U}}(x)-\mathbb{R}_{\geqq}^{k}\right)$ for all $x \in \mathcal{X}$. Figure 3.2 shows $\partial\left(\bar{z}(x)+\mathbb{R}_{\geq}^{k}\right), \partial\left(z_{\mathcal{U}}(x)-\mathbb{R}_{\geqq}^{k}\right)$ and $g(r, \lambda)$ for an example (Publication 3, Example 6). With help of the intersection points, it is easy to see that for $r=(0,1)^{T}, \lambda=(3,4)^{T}, x^{1}$ is uniquely optimal for $\mathrm{P}-\max (r, \lambda)$ and $x^{2}$ is uniquely optimal for $\mathrm{P}-\min (r, \lambda)$.

In addition, Figure $3.2(\mathrm{~b})$ shows the set $\tilde{Y}$ of all points in $Y:=\bigcup_{x \in \mathcal{X}} \partial\left(z_{\mathcal{U}}(x)-\mathbb{R}_{\geqq}^{k}\right)$, such that no $y^{\prime} \in Y$ with $y^{\prime}<y$ exists. For every point in $\tilde{Y}$, there exist $x \in \mathcal{X}$, $r \in \mathbb{R}^{k}, \lambda \in \mathbb{R}_{>}^{k}$ such that $(x, y)$ is an efficient solution for $\mathrm{G}-\min (r, \lambda)$.

\section{Minmax Robust Efficient Solutions Found with the Min-Ordering and Max-Ordering Scalarization Methods}

The authors show that optimal solutions for $\mathrm{P}-\max (r, \lambda)$ are pointMR weakly efficient for MOUP and that every pointMR weakly efficient solution for MOUP can be found with the max-ordering scalarization method. 


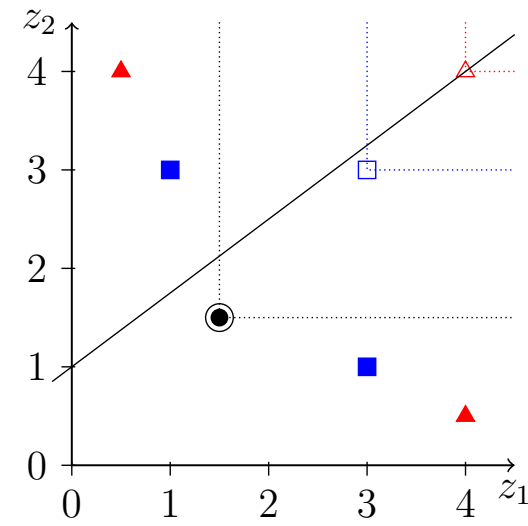

(a) Finding sol. for $\mathrm{P}-\max (r, \lambda)$

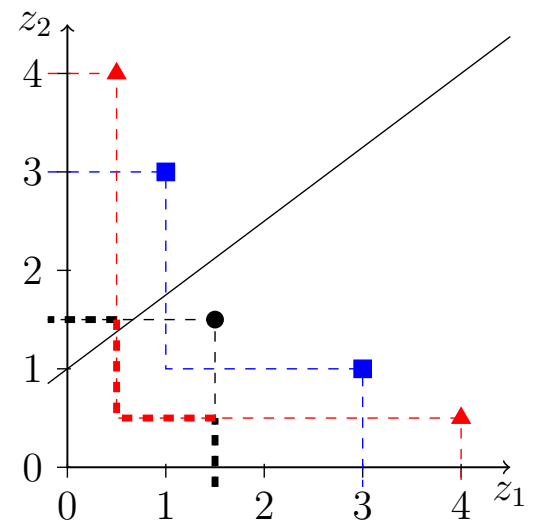

(b) Finding sol. for $\mathrm{P}-\min (r, \lambda)$

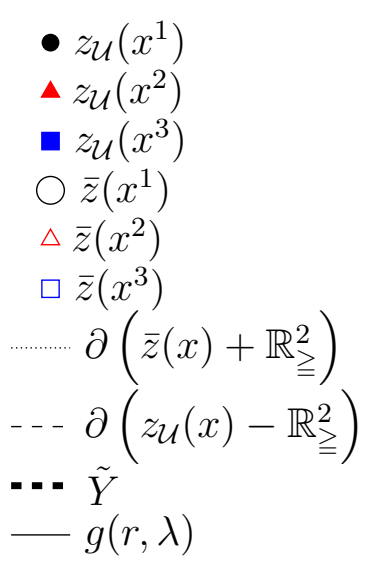

- $z_{\mathcal{U}}\left(x^{1}\right)$

$\Delta z_{\mathcal{U}}\left(x^{2}\right)$

- $z_{\mathcal{U}}\left(x^{3}\right)$

$\bar{z}\left(x^{1}\right)$

$\triangle \bar{z}\left(x^{2}\right)$

$\bar{z}\left(x^{3}\right)$

$\geqq)$

$\tilde{Y}$

$-g(r, \lambda)$

Figure 3.2.: Determining the intersection point of $g(r, \lambda)$ with $\partial\left(\bar{z}(x)+\mathbb{R}_{\geqq}^{2}\right)$ in (a) and $\partial\left(z_{\mathcal{U}}(x)-\mathbb{R}_{\geq}^{2}\right)$ in (b); as an example, $g(r, \lambda)$ is shown for $r=(0,1)^{T}$, $\lambda=(3,4)^{T}$ (Publication 3, Figure 3).

Theorem 3.3 (Publication 3, Theorem 11). Let $r \in \mathbb{R}^{k}, \lambda \in \mathbb{R}_{>}^{k}$ be given and let $x$ be an optimal solution for $P-\max (r, \lambda)$. Then

1. $x$ is a pointMR weakly efficient solution for MOUP and

2. if $x$ is the unique optimal solution for $P-\max (r, \lambda)$, then $x$ is a pointMR strictly efficient solution for MOUP.

Theorem 3.4 (Publication 3, Theorem 12). Let $x$ be a pointMR weakly efficient solution for MOUP and let a reference point $r \in \mathbb{R}^{k}$ with $r_{i}<\max _{\xi \in \mathcal{U}} z_{i}(x, \xi)$ $\forall i \in\{1, \ldots, k\}$ be given. Then there exists a weight vector $\lambda \in \mathbb{R}_{>}^{k}$ such that $x$ is an optimal solution for $P-\max (r, \lambda)$.

The min-ordering scalarization method, on the other hand, finds setMR weakly efficient solutions.

Theorem 3.5 (Publication 3, Theorem 13). Let $r \in \mathbb{R}^{k}, \lambda \in \mathbb{R}_{>}^{k}$ be given and let $x$ be an optimal solution for $P-\min (r, \lambda)$. Then

1. $x$ is a setMR weakly efficient solution for MOUP and

2. if $x$ is the unique optimal solution for $P$-min $(r, \lambda)$, then $x$ is a setMR strictly efficient solution for MOUP.

Moreover, the authors show that optimal solutions for $\mathrm{P}-\min (r, \lambda)$ cannot necessarily be found with any of the other known scalarization methods for finding setMR (weakly) efficient solutions, namely the weighted sum, $\epsilon$-constraint, (augmented) 
weighted Chebychev, p-norm or max-ordering scalarization method (Publication 3, Theorem 14). That is, with help of the min-ordering optimization problem one can find "new" setMR efficient solutions. In the example shown in Figure 3.2, the solution $x^{2}$ is optimal for $\mathrm{P}-\min (r, \lambda)$, but only $x^{1}$ is found with the other methods, whereas $x^{3}$ is neither found with the min-ordering method nor with any of the other scalarization methods.

The authors remark that even though $\mathrm{P}-\min (r, \lambda)$ could be easily transformed to a (single-objective) adjustable robust problem, a potential structure of the underlying problem (e.g., being a linear problem or a particular combinatorial problem) would often be lost.

\section{Min-Ordering and Max-Ordering Problems for MOUCO with Interval Uncertainty}

Furthermore, the authors of Publication 3 investigate how to approach $\mathrm{P}-\min (r, \lambda)$ and $\mathrm{P}-\max (r, \lambda)$ for multi-objective uncertain combinatorial optimization problems with particular uncertainty sets. They represent the solutions of a combinatorial optimization problem as binary vectors $x \in\{0,1\}^{n}$, as explained in Section 2.3.

First, they consider the straightforward multi-objective extension of interval uncertainty

$$
\mathcal{U}^{I}:=\left\{c \in \mathbb{R}^{k \times n}: c_{i, j}=\hat{c}_{i, j}+\beta_{i, j} \delta_{i, j}, \beta_{i, j} \in[0,1] \forall i \in\{1, \ldots, k\}, j \in\{1, \ldots, n\}\right\},
$$

with given minimal values $\hat{c}_{i, j} \in \mathbb{R}$ and interval lengths $\delta_{i, j} \in \mathbb{R}_{\geqq \text {. They show that }}$ instead of considering $\mathcal{U}^{I}$, it is sufficient to solve the problem with an uncertainty set only containing one scenario $\bar{c}$, defined by $\bar{c}_{i, j}:=\hat{c}_{i, j}+\delta_{i, j}$. It follows that $\mathrm{P}-\min (r, \lambda)$ can be solved in polynomial time, if the underlying single-objective deterministic problems $\min _{x \in \mathcal{X}} z_{i}(x, c)$ are polynomially solvable, while $\mathrm{P}-\max (r, \lambda)$ is NP-hard for many combinatorial problems (e.g., the shortest path or minimum spanning tree problem).

\section{Min-Ordering and Max-Ordering Problems for MOUCO with Bounded Uncertainty}

The authors introduce an extension of bounded uncertainty (Definition 2.6) to the multi-objective case, where the number of all uncertain parameters deviating from their nominal value is bounded (instead of regarding each objective independently as in $\mathcal{U}^{\text {owb }}$ in Definition 2.15). As they show later, in contrast to the problems and uncertainty sets considered in Bertsimas and Sim (2003); Hassanzadeh et al. (2013); Raith et al. (2018b), for P-min $(r, \lambda)$ they need to distinguish between integer and real valued factors $\beta_{i, j}$.

Definition 3.6 (Publication 3, Definitions 18 and 19). Let $\hat{c} \in \mathbb{R}^{k \times n}, \delta \in \mathbb{R}_{\geq}^{k \times n}$ and $\Gamma \in \mathbb{Z}$ with $0 \leqq \Gamma \leqq(k \cdot n)$ be given. We define the discretely bounded uncertainty 
set as

$$
\begin{array}{r}
\mathcal{U}^{d}:=\left\{c \in \mathbb{R}^{k \times n}: c_{i, j}=\hat{c}_{i, j}+\beta_{i, j} \delta_{i, j}, \beta_{i, j} \in\{0,1\} \forall i \in\{1, \ldots, k\}, j \in\{1, \ldots, n\},\right. \\
\left.\sum_{i \in\{1, \ldots, k\}, j \in\{1, \ldots, n\}} \beta_{i, j} \leqq \Gamma\right\}
\end{array}
$$

and the continuously bounded uncertainty set as

$$
\begin{array}{r}
\mathcal{U}^{c}:=\left\{c \in \mathbb{R}^{k \times n}: c_{i, j}=\hat{c}_{i, j}+\beta_{i, j} \delta_{i, j}, \beta_{i, j} \in[0,1] \forall i \in\{1, \ldots, k\}, j \in\{1, \ldots, n\},\right. \\
\left.\sum_{i \in\{1, \ldots, k\}, j \in\{1, \ldots, n\}} \beta_{i, j} \leqq \Gamma\right\} .
\end{array}
$$

For these uncertainty sets, the authors of Publication 3 develop compact MILP formulations for $\mathrm{P}-\max (r, \lambda)$ and $\mathrm{P}-\min (r, \lambda)$, i.e., formulations without any inner maximum or minimum function.

First, the authors show that for $\mathrm{P}-\max (r, \lambda)$ one does not need to distinguish between $\mathcal{U}^{d}$ and $\mathcal{U}^{c}$, and that both can even be replaced by an objective-wise bounded uncertainty set $\mathcal{U}^{\text {owb }}$ with $\Gamma_{1}=\ldots=\Gamma_{k}$ (Publication 3 , Lemma 21).

They conclude that to obtain a compact formulation for $\mathrm{P}-\max (r, \lambda)$ with uncertainty set $\mathcal{U}^{d}$ or $\mathcal{U}^{c}$, the same approach as in Hassanzadeh et al. (2013) for linear problems with uncertainty set $\mathcal{U}^{\text {owb }}$ can be applied: The authors substitute the inner maximum functions in the objective function by $k$ constraints, which contain embedded optimization problems $\max _{c \in \mathcal{U}} \lambda_{i}\left(z_{i}(x, c)-r_{i}\right)$. Then, they replace each of these embedded problems with its dual in the form introduced by Bertsimas and Sim (2003), obtaining a compact MILP formulation for $\mathrm{P}-\max (r, \lambda)$.

For $\mathrm{P}-\min (r, \lambda)$ with continuously bounded uncertainty set $\mathcal{U}^{c}$ the authors proceed in a similar way: they find a linear programming formulation of the inner problem $\max _{c \in \mathcal{U}^{c}} \min _{i \in\{1, \ldots, k\}} \lambda_{i}\left(z_{i}(x, c)-r_{i}\right)$ and dualize it, using the results by Bertsimas and $\operatorname{Sim}(2003)$.

The same approach is not suitable for $\mathrm{P}-\min (r, \lambda)$ with discretely bounded uncertainty set $\mathcal{U}^{d}$, because the inner maximization problem is not a linear program, and is not equivalent to its linear relaxation, which is identical to the inner problem obtained for $\mathcal{U}^{c}$ (see Publication 3, Example 22). Nevertheless, the authors develop a compact MILP formulation for P-min $(r, \lambda)$ with help of the identity in Theorem 3.8.

Definition 3.7 (Publication 3, Definitions 23 and 25). Let $\delta$ be a vector in $\mathbb{R}^{n}$ or a matrix in $\mathbb{R}^{k \times l}$ and let an index set $I \subseteq\{1, \ldots, n\}$ resp. $I \subseteq\{1, \ldots, k\} \times\{1, \ldots, l\}$ 
be given. We denote the $h$-smallest of all entries $\delta_{j}$ with $j \in I$ as $h$ - $\min _{I} \delta$ and the $h$-greatest as $h-\max _{I} \delta$. For $h=0$ or $h>|I|$ we set $h-\min _{I} \delta=h$ - $\max _{I} \delta=0$. Let $r \in \mathbb{R}^{k}, \lambda \in \mathbb{R}_{>}^{k}$ and $x \in \mathcal{X}$ be given. We define $M(x) \in \mathbb{R}^{k \times(\Gamma+1)}$ by its entries

$$
m_{i, l}:=\lambda_{i}\left(-r_{i}+\sum_{j \in I(x)} \hat{c}_{i, j}+\sum_{h=1}^{l-1} h-\max _{I(x)} \delta_{(i, \cdot)}\right),
$$

where $I(x):=\left\{j \in\{1, \ldots, n\}: x_{j}=1\right\}$. That is, $\frac{m_{i, l}+r_{i}}{\lambda_{i}}$ is the sum of the nominal cost of $x$ in the $i$-th objective and the l highest interval lengths $\delta_{i, j}$ among those with $x_{j}=1$ w.r.t. the $i$-th objective.

Theorem 3.8 (Publication 3, Theorem 27). Given $x \in \mathcal{X}$ and the corresponding matrix $M(x)$, the optimal objective value $z^{*}$ of the inner maximization problem of $P$-min $(r, \lambda)$ equals the $(\Gamma+1)$-smallest entry in $M(x)$, i.e.,

$$
z^{*}:=\max _{c \in \mathcal{U}^{d}} \min _{i \in\{1, \ldots, k\}} \lambda_{i}\left(z_{i}(x, c)-r_{i}\right)=(\Gamma+1)-\min _{\{1, \ldots, k\} \times\{1, \ldots, \Gamma+1\}} M(x)=: m^{*} .
$$

The authors formulate a compact mixed integer linear program that minimizes the $(\Gamma+1)$-smallest entry in $M(x)$ over all $x \in \mathcal{X}$, which hence is a compact MILP formulation for $\mathrm{P}-\min (r, \lambda)$ with discretely bounded uncertainty set.

Further, the authors show that the complexity of $\mathrm{P}-\min (r, \lambda)$ and $\mathrm{P}-\max (r, \lambda)$ with bounded uncertainty depends on $\Gamma$. For $\Gamma=0$ the uncertainty set only contains one scenario and $\mathrm{P}-\max (r, \lambda)$ is already NP-hard for many combinatorial problems, as the shortest path and minimum spanning tree problem, whereas $\mathrm{P}-\min (r, \lambda)$ is polynomially solvable as long as the underlying deterministic problem is polynomially solvable. However, for $\Gamma=1, \mathrm{P}-\min (r, \lambda)$ with discretely bounded uncertainty set is NP-hard for the shortest path and minimum spanning tree problem, which is shown by reducing the single-objective minmax robust problem with discrete uncertainty set to $\mathrm{P}-\min (r, \lambda)$ (Publication 3, Theorem 29).

\section{Own contribution}

This manuscript is joint work with M. Schmidt and A. Schöbel. The initial idea for the scalarizations was contributed by M. Schmidt, but the ideas for most theorems and solution approaches were elaborated jointly by the authors. The idea for the geometrical characterization and the proof that $\mathrm{P}-\min (r, \lambda)$ finds "new" setMR efficient solutions is my own work. A big part of the details, including most of the proofs, models, examples and figures, as well as the major part of the writing, were done by myself with consultation of the other authors. 


\section{Discussion}

Combinatorial problems have been extensively studied in multi-objective optimization and robust optimization, yet hardly any approaches to find robust efficient solutions for multi-objective uncertain combinatorial problems have existed previous to the work presented in this thesis. The field of multi-objective robust optimization is currently gaining more and more interest, as many of the concepts of robust efficiency have been developed during the last years. Moreover, there is an inherent difficulty to multi-objective robust optimization, as it combines challenges arising from both the uncertainties and the multi-objective nature of the problems.

This thesis contributes to the analysis of robust efficient solutions and the development of solution methods for multi-objective uncertain combinatorial problems, considering various types of uncertainty and concepts of robust efficiency. Approaches from both robust optimization and multi-objective optimization are extended and combined, using properties of particular robustness concepts and uncertainty sets.

Beyond solution approaches for general combinatorial problems (see Publications 1 and 3) the shortest path problem is considered in particular (Publications 1 and 2). The shortest path problem is an example for an "easy" problem becoming "hard" in multi-objective robust optimization: it is polynomially solvable in the single-objective deterministic case, but its multi-objective counterpart can have exponentially many nondominated objective vectors and it is, e.g., NP-hard in the minmax robust case with finite uncertainty.

Among the various existing concepts for robust efficiency, a focus is put on pointMR and setMR efficiency, which both extend the popular single-objective concept of minmax robustness. These concepts are considered in all three publications constituting the cumulative part of this thesis. The authors of Publication 1 use the concept of pointMR efficiency, and, since the uncertainty set in this setting is an objectivewise uncertainty set, all pointMR efficient solutions are also setMR efficient and vice versa (see Lemma 2.13). With the scalarization methods introduced in Publication 3, setMR respective pointMR efficient solutions are found. In Publication 1, too, both concepts are considered, as well as flimsily and highly robust efficiency and multiscenario efficiency.

Furthermore, different uncertainty sets are regarded. Finite uncertainty (considered in Publication 2) and interval uncertainty (considered in Publication 3) are straightforward extensions of the respective uncertainty sets in single-objective robust optimization. The single-objective concept of bounded uncertainty can be extended to 
multi-objective robust optimization in several ways. In Publication 1 an objectivewise variant is considered, and the authors of Publication 3 introduce the continuously and discretely bounded uncertainty sets.

Table 4.1 gives an overview of the considered problems, robustness concepts and uncertainty sets in the cumulative part of this thesis. For practical applications with any

\begin{tabular}{|l|lll|}
\hline Publication & Problems & Concepts & Uncertainty Sets \\
\hline Publication 1 & CO, SP & pointMR, (setMR) & objective-wise bounded \\
Publication 2 & SP & $\begin{array}{l}\text { pointMR, setMR, } \\
\text { flimsily, highly, } \\
\text { multi-scenario } \\
\text { pointMR, setMr }\end{array}$ & finite \\
Publication 3 & G, CO & $\begin{array}{l}\text { discretely/continuously bounded, } \\
\text { interval }\end{array}$ \\
\hline
\end{tabular}

Table 4.1.: Considered problem classes (G: general optimization problems, CO: general combinatorial problems, SP: the shortest path problem), concepts of robust efficiency and uncertainty sets.

of the combinations of problem type, robustness concept and uncertainty set listed in Table 4.1, a decision maker can now choose an appropriate solution method from the publications summarized in this thesis. In case of several different approaches for the same combination, the authors compare their developed algorithms experimentally and recommend one or another approach depending on the parameters of the problem (see Publications 1 and 2).

Moreover, this thesis indicates how to approach further multi-objective robust uncertain combinatorial optimization problems by showing strategies to find new solution methods based on results from different fields.

On the one hand, algorithms for finding minmax robust solutions for single-objective uncertain problems are analyzed and extended to find pointMR and setMR efficient solutions in Publications 1 and 2.

On the other hand, solution methods based on ideas from multi-objective optimization are developed. In Publication 3, the authors introduce two scalarization methods to obtain pointMR or setMR efficient solutions by solving several scalarized problems, which is a common approach in multi-objective optimization. In Publications 1 and 2, labeling algorithms for the multi-objective deterministic shortest path problem are extended to the multi-objective robust case, sometimes combined with algorithms or solution ideas from robust optimization.

Furthermore, the structure of particular uncertainty sets is used to formulate mixed integer linear programming models for the scalarized problems in Publication 3.

This shows that the combination of uncertainties and multiple objectives leads not 
only to very challenging combinatorial problems, but also offers a variety of possible solution approaches.

An advantage of adapting algorithms from robust optimization or multi-objective optimization is that theoretical complexity results can often be retained or derived: For example, the multi-objective labeling algorithm extended in Publication 2 runs in pseudo-polynomial time under some assumptions, as do all presented extensions. Further, the runtime of the algorithms repeatedly applying an algorithm for multiobjective deterministic problems (see Publications 1 and 2) is bounded by a multiple of that algorithm's runtime (plus the time for filtering the solutions, if necessary). This also means that these algorithms directly profit from any advances in the field of multi-objective optimization. The approaches in Publication 1 have the additional advantage that they are valid for all combinatorial problems, and that the subproblems are of the same kind as the original problem, hence specific algorithms for specific combinatorial problems can be applied. On the other hand, the scalarization approach given in Publication 3 can be used for all uncertainty sets, as long as a suitable method for solving the scalarized problems is found.

Furthermore, the solution methods presented in this thesis show that similar approaches are suitable for different robustness concepts and uncertainty sets. For example, labeling algorithms have been used in several of the methods for the shortest path problem, either by extending them or using them to solve auxiliary problems. The scalarization methods introduced in Publication 3 can also be applied to the problems in Publications 1 and 2: The MILP formulation for $\mathrm{P}-\max (r, \lambda)$ with discretely and continuously bounded uncertainty is also valid for objective-wise bounded uncertainty as considered in Publication 1, if the $\Gamma_{i}$ are identical. Compact MILP formulations can be obtained for $\mathrm{P}-\min (r, \lambda)$ and $\mathrm{P}-\max (r, \lambda)$ with a finite uncertainty set, too, and can hence be used to find pointMR and setMR efficient solutions for the shortest path problem in Publication 2.

In conclusion, different uncertainty sets and robustness concepts require different solution methods, but often similar approaches can be used to find these methods. Therefore, approaches introduced in this thesis can likely be of help when developing solution methods for problems with other robustness concepts and uncertainty sets. These approaches comprise the extension of algorithms from robust and multiobjective optimization and mixed integer programming formulations. A general superiority of one approach over the others is not observed. 



\section{Conclusion and Future Work}

In this thesis, several solution methods for multi-objective robust combinatorial optimization problems have been developed. In Section 3.1, two approaches for finding pointMR (resp. setMR) efficient solutions for multi-objective combinatorial problems with objective-wise bounded uncertainty have been introduced and applied to the shortest path problem. In Section 3.2, we presented labeling algorithms for the multi-objective shortest path problem with finite uncertainty, finding robust efficient solutions according to the concepts of multi-scenario efficiency, flimsily and highly robust efficiency and pointMR and setMR efficiency. Two scalarization methods for finding pointMR and setMR efficient solutions were introduced in Section 3.3, and approaches to solve the scalarized problems were shown for interval uncertainty and discretely and continuously bounded uncertainty.

All proposed solution methods provide specific issues for future work. For example, it could be worthwhile to investigate whether acceleration methods for labeling algorithms can be applied to the algorithms presented in Sections 3.1 and 3.2, or to combine the scalarization methods from Section 3.3 by using ordered median functions as scalarizing functions.

Furthermore, a great variety of robustness concepts for multi-objective optimization exists, as indicated in Section 2.3. We focused on pointMR and setMR efficiency, and also regarded flimsily and highly robust efficiency and multi-scenario efficiency. Considering different robustness concepts leads to different problems to solve and hence requires different solution methods, as shown for the shortest path problem in Section 3.2. Therefore, one aspect of future work should be to analyze whether solution methods presented in this thesis can be adapted to further concepts of robust efficiency or whether new approaches are needed.

Similarly, the assumed uncertainty set plays an important role for the structure and complexity of the resulting robust problem. We have considered general combinatorial problems with interval uncertainty and several versions of bounded uncertainty, and shortest path problems with objective-wise bounded and finite uncertainty. An interesting research question would be whether the proposed solution methods can be used or adapted for other uncertainty sets, including ellipsoidal, polyhedral and other variants of bounded uncertainty.

The methods presented in Chapter 3 are based on approaches from multi-objective optimization, robust optimization and integer programming. We are far from exhausting the results and methods from these long-established fields, leaving much opportunity for further solution approaches for multi-objective robust combinatorial 
problems.

Apart from developing methods for general combinatorial problems, we have shown how to extend specific algorithms for the (robust or multi-objective) shortest path problem to the multi-objective robust case. It is of interest whether other solution methods for the shortest path problem could be extended as well. Specific algorithms for other combinatorial problems, e.g., the multi-objective robust minimum spanning tree problem, are yet to be developed.

In conclusion, covering several robustness concepts and uncertainty sets and providing a variety of solution methods, this thesis gives an insight into the challenges of multi-objective robust combinatorial optimization and how to approach them. It is one of the first contributions on the way to an extensive analysis of and solution concept for multi-objective robust combinatorial optimization. As emphasized in the introduction and the application sections, this is also of practical relevance, since real-world optimization problems are often of a multi-objective and uncertain nature. 


\section{Bibliography}

Avigad, G. and Branke, J. (2008). Embedded evolutionary multi-objective optimization for worst case robustness. In Proceedings of the 10th Annual Conference on Genetic and Evolutionary Computation (GECCO '08), pages 617-624. ACM, New York.

Bellman, R. (1958). On a routing problem. Quarterly of Applied Mathematics, 16(1):87-90.

Ben-Tal, A., El Ghaoui, L., and Nemirovski, A. (2009). Robust optimization. Princeton University Press, Princeton and Oxford.

Ben-Tal, A., Goryashko, A., Guslitzer, E., and Nemirovski, A. (2004). Adjustable robust solutions of uncertain linear programs. Mathematical Programming, 99(2):351376.

Ben-Tal, A. and Nemirovski, A. (1998). Robust convex optimization. Mathematics of Operations Research, 23(4):769-805.

Bertsimas, D. and Sim, M. (2003). Robust discrete optimization and network flows. Mathematical Programming, 98(1):49-71.

Bertsimas, D. and Sim, M. (2004). The price of robustness. Operations Research, $52(1): 35-53$.

Bitran, G. R. (1980). Linear multiple objective problems with interval coefficients. Management Science, 26(7):694-706.

Bokrantz, R. and Fredriksson, A. (2017). Necessary and sufficient conditions for Pareto efficiency in robust multiobjective optimization. European Journal of Operational Research, 262(2):682-692.

Botte, M. and Schöbel, A. (2016). Dominance for multi-objective robust optimization. Technical Report 2016-8, Preprint-Reihe, Institut für Numerische und Angewandte Mathematik, Universität Göttingen.

Bowman, V. J. (1976). On the relationship of the Tchebycheff norm and the efficient frontier of multiple-criteria objectives. In Thiriez, H. and Zionts, S., editors, Multiple Criteria Decision Making, volume 130 of Lecture Notes in Economics and 
Mathematical Systems (Operations Research), pages 76-86. Springer, Berlin, Heidelberg.

Büsing, C. and D'Andreagiovanni, F. (2014). A new theoretical framework for robust optimization under multi-band uncertainty. In Helber, S., Breitner, M., Rösch, D., Schön, C., Graf von der Schulenburg, J.-M., Sibbertsen, P., Steinbach, M., Weber, S., and Wolter, A., editors, Operations Research Proceedings 2012, pages 115-121. Springer, Cham.

Chankong, V. and Haimes, Y. Y. (1983). Multiobjective Decision Making: Theory and Methodology. Number 8 in North Holland Series in System Science and Engineering. Elsevier, New York.

Chassein, A., Dokka, T., and Goerigk, M. (2018). Algorithms and uncertainty sets for data-driven robust shortest path problems. ArXiv e-prints. https://arxiv.org/abs/1802.04149v1.

Cicerone, S., D’Angelo, G., Di Stefano, G., Frigioni, D., and Navarra, A. (2007). Robust algorithms and price of robustness in shunting problems. In Liebchen, C., Ahuja, R. K., and Mesa, J. A., editors, 7th Workshop on Algorithmic Approaches for Transportation Modeling, Optimization, and Systems (ATMOS07), volume 7 of OpenAccess Series in Informatics (OASIcs), pages 175-190. Schloss DagstuhlLeibniz-Zentrum fuer Informatik, Dagstuhl, Germany.

Cintrano, C., Chicano, F., and Alba, E. (2017). Robust bi-objective shortest path problem in real road networks. In Alba, E., Chicano, F., and Luque, G., editors, Smart Cities, pages 128-136. Springer, Cham.

Corley, H. and Moon, I. (1985). Shortest paths in networks with vector weights. Journal of Optimization Theory and Applications, 46(1):79-86.

Deb, K. and Gupta, H. (2006). Introducing robustness in multi-objective optimization. Evolutionary Computation, 14(4):463-494.

Dijkstra, E. (1959). A note on two problems in connexion with graphs. Numerische Mathematik, 1:269-271.

Doolittle, E., Kerivin, H. M., and Wiecek, M. M. (2012). A robust multiobjective optimization problem with application to internet routing. Technical Report R201211-DKW, Clemson University.

Doolittle, E. K., Dranichak, G. M., Muir, K., and Wiecek, M. M. (2016). A note on robustness of the min-max solution to multi-objective linear programs. International Journal of Multicriteria Decision Making, 6(4):343-365. 
Edgeworth, F. Y. (1881). Mathematical psychics: An essay on the application of mathematics to the moral sciences. C. Kegan Paul \& Co., London.

Ehrgott, M. (2005). Multicriteria optimization. Springer, Berlin, Heidelberg.

Ehrgott, M. (2006). A discussion of scalarization techniques for multiple objective integer programming. Annals of Operations Research, 147(1):343-360.

Ehrgott, M. and Gandibleux, X. (2000). A survey and annotated bibliography of multiobjective combinatorial optimization. OR Spectrum, 22(4):425-460.

Ehrgott, M., Ide, J., and Schöbel, A. (2014). Minmax robustness for multi-objective optimization problems. European Journal of Operational Research, 239(1):17-31.

Eichfelder, G., Krüger, C., and Schöbel, A. (2017). Decision uncertainty in multiobjective optimization. Journal of Global Optimization, 69(2):485-510.

Erera, A. L., Morales, J. C., and Savelsbergh, M. (2009). Robust optimization for empty repositioning problems. Operations Research, 57(2):468-483.

Fischetti, M. and Monaci, M. (2009). Light robustness. In Ahuja, R. K., Möhring, R. H., and Zaroliagis, C. D., editors, Robust and Online Large-Scale Optimization: Models and Techniques for Transportation Systems, pages 61-84. Springer, Berlin, Heidelberg.

Fliege, J. and Werner, R. (2014). Robust multiobjective optimization \& applications in portfolio optimization. European Journal of Operational Research, 234(2):422433 .

Gass, S. and Saaty, T. (1955). The computational algorithm for the parametric objective function. Naval Research Logistics, 2(1-2):39-45.

Goerigk, M. and Schöbel, A. (2016). Algorithm engineering in robust optimization. In Kliemann, L. and Sanders, P., editors, Algorithm Engineering: Selected Results and Surveys, volume 9220 of Lecture Notes in Computer Science, pages 245-279. Springer, Cham.

Guerriero, F. and Musmanno, R. (2001). Label correcting methods to solve multicriteria shortest path problems. Journal of Optimization Theory and Applications, 111(3):589-613.

Gunawan, S. and Azarm, S. (2005). Multi-objective robust optimization using a sensitivity region concept. Structural and Multidisciplinary Optimization, 29(1):5060 . 
Haimes, Y. Y., Lasdon, L. S., and Wismer, D. A. (1971). On a bicriterion formulation of the problems of integrated system identification and system optimization. IEEE Transactions on Systems, Man, and Cybernetics, 1(3):296-297.

Hamacher, H. W. and Ruhe, G. (1994). On spanning tree problems with multiple objectives. Annals of Operations Research, 52(4):209-230.

Hansen, P. (1980). Bicriterion path problems. In Fandel, G. and Gal, T., editors, Multiple Criteria Decision Making Theory and Application, volume 177 of Lecture Notes in Economics and Mathematical Systems, pages 109-127. Springer, Berlin, Heidelberg.

Hassanzadeh, F., Nemati, H., and Sun, M. (2013). Robust optimization for multiobjective programming problems with imprecise information. Procedia Computer Science, 17:357-364.

Hombach, L. E., Büsing, C., and Walther, G. (2017). Robust and sustainable supply chains under market uncertainties and different risk attitudes - A case study of the german biodiesel market. European Journal of Operational Research.

Iancu, D. A. and Trichakis, N. (2014). Pareto efficiency in robust optimization. Management Science, 60(1):130-147.

Ide, J. (2014). Concepts of Robustness for Uncertain Multi-Objective Optimization. PhD thesis, Universität Göttingen.

Ide, J., Köbis, E., Kuroiwa, D., Schöbel, A., and Tammer, C. (2014). The relationship between multi-objective robustness concepts and set-valued optimization. Fixed Point Theory and Applications, 2014(83).

Ide, J. and Schöbel, A. (2016). Robustness for uncertain multi-objective optimization: A survey and analysis of different concepts. OR Spectrum, 38(1):235-271.

Ide, J., Tiedemann, M., Westphal, S., and Haiduk, F. (2015). An application of deterministic and robust optimization in the wood cutting industry. $4 O R, 13(1): 35-$ 57.

Iori, M., Martello, S., and Pretolani, D. (2010). An aggregate label setting policy for the multi-objective shortest path problem. European Journal of Operational Research, 207(3):1489-1496.

Kasperski, A. and Zieliński, P. (2016). Robust discrete optimization under discrete and interval uncertainty: A survey. In Robustness Analysis in Decision Aiding, Optimization, and Analytics, pages 113-143. Springer, Cham. 
Klamroth, K., Köbis, E., Schöbel, A., and Tammer, C. (2017). A unified approach to uncertain optimization. European Journal of Operational Research, 260(2):403-420.

Kouvelis, P. and Yu, G. (1997). Robust discrete optimization and its applications. Kluwer Academic Publishers, Boston.

Krüger, C. (2018a). On Minmax Robustness for Multiobjective Optimization with Decision or Parameter Uncertainty. PhD thesis, Universität Göttingen.

Krüger, C. (2018b). Peat and pots: Analysis of robust solutions for a biobjective problem in agriculture. Technical Report 2018-5, Preprint-Reihe, Institut für Numerische und Angewandte Mathematik, Universität Göttingen.

Krüger, C., Castellani, F., Geldermann, J., and Schöbel, A. (2018). Peat and pots: An application of robust multiobjective optimization to a mixing problem in agriculture. Technical Report 2018-4, Preprint-Reihe, Institut für Numerische und Angewandte Mathematik, Universität Göttingen.

Krüger, C., Schöbel, A., and Wiecek, M. M. (2017). The robustness gap for uncertain multiobjective optimization. Technical Report 2017-3, Preprint-Reihe, Institut für Numerische und Angewandte Mathematik, Universität Göttingen.

Kuhn, K., Raith, A., Schmidt, M., and Schöbel, A. (2016). Bi-objective robust optimisation. European Journal of Operational Research, 252(2):418-431.

Kuroiwa, D. and Lee, G. M. (2012). On robust multiobjective optimization. Vietnam Journal of Mathematics, 40(2-3):305-317.

Lee, T. and Kwon, C. (2014). A short note on the robust combinatorial optimization problems with cardinality constrained uncertainty. 4OR, 12(4):373-378.

Liebchen, C., Lübbecke, M., Möhring, R., and Stiller, S. (2009). The concept of recoverable robustness, linear programming recovery, and railway applications. In Ahuja, R., Möhring, R., and Zaroliagis, C., editors, Robust and Online Large-Scale Optimization: Models and Techniques for Transportation Systems, volume 5868 of Lecture Notes in Computer Science, pages 1-27. Springer, Berlin, Heidelberg.

Majewski, D. E., Wirtz, M., Lampe, M., and Bardow, A. (2017). Robust multiobjective optimization for sustainable design of distributed energy supply systems. Computers \& Chemical Engineering, 102:26-39.

Martins, E. (1984). On a multicriteria shortest path problem. European Journal of Operational Research, 16(2):236-245. 
Mavrotas, G., Figueira, J. R., and Siskos, E. (2015). Robustness analysis methodology for multi-objective combinatorial optimization problems and application to project selection. Omega, 52:142 - 155 .

Murthy, I. and Her, S. (1992). Solving min-max shortest-path problems on a network. Naval Research Logistics, 39(5):669-683.

Nikulin, Y., Karelkina, O., and Mäkelä, M. M. (2013). On accuracy, robustness and tolerances in vector Boolean optimization. European Journal of Operational Research, 224(3):449-457.

Paixão, J. M. and Santos, J. L. (2013). Labeling methods for the general case of the multi-objective shortest path problem-A computational study. In Madureira, A., Reis, C., and Marques, V., editors, Computational Intelligence and Decision Making, pages 489-502. Springer Netherlands, Dordrecht.

Pareto, V. (1896). Cours déconomie politique. F.Rouge, Lausanne.

Park, K.-C. and Lee, K.-S. (2007). A note on robust combinatorial optimization problem. Management Science and Financial Engineering, 13(1):115-119.

Poss, M. (2014). Robust combinatorial optimization with variable cost uncertainty. European Journal of Operational Research, 237(3):836-845.

Raith, A., Schmidt, M., Schöbel, A., and Thom, L. (2018a). Extensions of labeling algorithms for multi-objective uncertain shortest path problems. Networks.

Raith, A., Schmidt, M., Schöbel, A., and Thom, L. (2018b). Multi-objective minmax robust combinatorial optimization with cardinality-constrained uncertainty. European Journal of Operational Research, 267(2):628-642.

Schmidt, M., Schöbel, A., and Thom, L. (2018). Min-ordering and max-ordering scalarization methods for multi-objective robust optimization. Technical Report 2018-3, Preprint-Reihe, Institut für Numerische und Angewandte Mathematik, Universität Göttingen.

Schöbel, A. (2014). Generalized light robustness and the trade-off between robustness and nominal quality. Mathematical Methods of Operations Research, 80(2):161-191.

Soyster, A. L. (1973). Technical note - Convex programming with set-inclusive constraints and applications to inexact linear programming. Operations Research, 21(5):1154-1157.

Steuer, R. E. and Choo, E.-U. (1983). An interactive weighted Tchebycheff procedure for multiple objective programming. Mathematical Programming, 26(3):326-344. 
Wiecek, M. M., Blouin, V. Y., Fadel, G. M., Engau, A., Hunt, B. J., and Singh, V. (2009). Multi-scenario multi-objective optimization with applications in engineering design. In Barichard, V., Ehrgott, M., Gandibleux, X., and T'Kindt, V., editors, Multiobjective Programming and Goal Programming, volume 618 of Lecture Notes in Economics and Mathematical Systems, pages 283-298. Springer, Berlin, Heidelberg.

Wiecek, M. M. and Dranichak, G. M. (2016). Robust multiobjective optimization for decision making under uncertainty and conflict. In Gupta, A. and Capponi, A., editors, Optimization Challenges in Complex, Networked and Risky Systems, INFORMS Tutorials in Operations Research (J.C. Smith, ed.), chapter 4, pages 84-114. INFORMS.

Witting, K., Ober-Blöbaum, S., and Dellnitz, M. (2013). A variational approach to define robustness for parametric multiobjective optimization problems. Journal of Global Optimization, 57(2):331-345.

Yu, G. and Yang, J. (1998). On the robust shortest path problem. Computers \&6 Operations Research, 25(6):457-468.

Yu, H. and Liu, H. M. (2013). Robust multiple objective game theory. Journal of Optimization Theory and Applications, 159(1):272-280. 



\section{A. Publications}

\section{A.1. Multi-Objective Minmax Robust Combinatorial Optimization with Cardinality-Constrained Uncertainty}

published in the European Journal of Operational Research (Raith et al., 2018b)

Authors: Andrea Raith, Marie Schmidt, Anita Schöbel, Lisa Thom 


\title{
Multi-objective minmax robust combinatorial optimization with cardinality-constrained uncertainty
}

\author{
Andrea Raith ${ }^{\mathrm{a}}$, Marie Schmidt ${ }^{\mathrm{b}}$, Anita Schöbel ${ }^{\mathrm{c}}$, Lisa Thom ${ }^{\mathrm{c}, *}$ \\ a Department of Engineering Science, The University of Auckland, Private Bag 92019, Auckland 1142, New Zealand \\ bepartment of Technology and Operations Management, Rotterdam School of Management, Erasmus University Rotterdam, PO Box 1738, 3000 DR \\ Rotterdam, The Netherlands \\ 'Institut für Numerische und Angewandte Mathematik, Universität Göttingen, Lotzestr. 16-18, 37083 Göttingen, Germany
}

\section{A R T I C L E I N F O}

Article history:

Received 19 December 2016

Accepted 11 December 2017

Available online 18 December 2017

\section{Keywords:}

Multiple objective programming

Robust optimization

Combinatorial optimization

Multi-objective robust optimization

Shortest path problem

\begin{abstract}
A B S T R A C T
In this paper, we develop two approaches to find minmax robust efficient solutions for multi-objective combinatorial optimization problems with cardinality-constrained uncertainty. First, we extend an existing algorithm for the single-objective problem to multi-objective optimization. We propose also an enhancement to accelerate the algorithm, even for the single-objective case, and we develop a faster version for special multi-objective instances. Second, we introduce a deterministic multi-objective problem with sum and bottleneck functions, which provides a superset of the robust efficient solutions. Based on this, we develop a label setting algorithm to solve the multi-objective uncertain shortest path problem. We compare both approaches on instances of the multi-objective uncertain shortest path problem originating from hazardous material transportation.
\end{abstract}

(c) 2017 Elsevier B.V. All rights reserved.

\section{Introduction}

Two of the main difficulties in applying optimization techniques to real-world problems are that several (conflicting) objectives may exist and that parameters may not be known exactly in advance. In multi-objective optimization several objectives are optimized simultaneously by choosing solutions that cannot be improved in one objective without worsening it in another objective. Robust optimization hedges against (all) possible parameter values, e.g., by assuming the worst case for each solution (minmax robustness).

Often it is assumed that the uncertain parameters take any value from a given interval or that discrete scenarios are given. A survey on robust combinatorial optimization with these uncertainty sets is given by Aissi, Bazgan, and Vanderpooten (2009). Based on the interval case, Bertsimas and Sim (2004) propose to consider scenarios where only a bounded number of parameters differ from their expected value (cardinality-constrained uncertainty). This leads to less conservative solutions that are of high practical use. Bertsimas and Sim (2003) provide an algorithm to find robust solutions for combinatorial optimization problems under this kind of uncertainty.

\footnotetext{
Corresponding author

E-mail addresses: a.raith@auckland.ac.nz (A. Raith), schmidt2@rsm.nl (M. Schmidt), schoebel@math.uni-goettingen.de (A. Schöbel), l.thom@math.unigoettingen.de (L. Thom).

https://doi.org/10.1016/j.ejor.2017.12.018

0377-2217/@ 2017 Elsevier B.V. All rights reserved.
}

Only recently have robust optimization concepts for multiobjective problems been developed. Kuroiwa and Lee (2012) and Fliege and Werner (2014) introduce a first extension of minmax robustness for several objectives. They consider the uncertainties in the objectives independently of each other. Ehrgott, Ide, and Schöbel (2014) develop another extension of minmax robustness, in which they include the dependencies between the objectives. This is further generalized by Ide, Köbis, Kuroiwa, Schöbel, and Tammer (2014). These concepts have been extensively applied, e.g., in portfolio management (Fliege \& Werner, 2014), in game theory (Yu \& Liu, 2013) and in the wood industry (Ide, Tiedemann, Westphal, \& Haiduk, 2015). Ide and Schöbel (2016) and Wiecek and Dranichak (2016) give an overview on multi-objective robustness, including further robustness concepts. Newest developments in this field include works by Chuong (2016) and Kalantari, Dong, and Davies (2016). Cardinality constrained uncertainty is extended to multi-objective optimization by Doolittle, Kerivin, and Wiecek (2012) (only for uncertain constraints) and Hassanzadeh, Nemati, and Sun (2013) (for uncertain objective functions and constraints).

To the best of our knowledge, only Kuhn, Raith, Schmidt, and Schöbel (2016) have developed a solution algorithm for multiobjective uncertain combinatorial optimization problems. They consider problems with two objectives, of which only one is uncertain, with discrete and polyhedral uncertainty sets.

In this paper, however, we consider problems with any fixed number of objectives of which all may be uncertain. The main

https://doi.org/10.1016/j.ejor.2017.12.018 
contribution of this paper is that we develop two solution approaches for multi-objective combinatorial optimization problems with cardinality-constrained uncertainty. We further derive specific algorithms for the multi-objective uncertain shortest path problem.

The remainder of this paper is structured as follows: in Section 2 we give a short introduction to multi-objective robust optimization. We present two solution approaches for multiobjective combinatorial optimization problems with cardinalityconstrained uncertainty in Section 3: in Section 3.1 we extend an algorithm by Bertsimas and $\operatorname{Sim}$ (2003) to multi-objective optimization. Additionally, we propose an acceleration for both the single-objective and the multi-objective case and a faster version for multi-objective problems with a special property. In Section 3.2, we introduce a second approach and show how it can be applied to solve the multi-objective uncertain shortest path problem as an example. In Section 4, we compare our methods on instances of the multi-objective uncertain shortest path problem originating from hazardous material transportation.

\section{Multi-objective combinatorial optimization with cardinality-constrained uncertainty}

First, we give an introduction to multi-objective combinatorial optimization. We use bold font for vectors and vector valued functions.

An instance $(E, Q, \boldsymbol{c})$ of a multi-objective combinatorial optimization problem is given by a finite element set $E$, a set $Q \subseteq 2^{E}$ of feasible solutions, which are subsets of $E$, and a cost function $\boldsymbol{c}$, that assigns a cost vector $\boldsymbol{c}(e)=\left(c_{1}(e), \ldots, c_{k}(e)\right)$ to each element $e \in E$. The cost $\boldsymbol{z}(q)$ of a set $q \in Q$ is the sum of the costs of its elements. We call

(MOCO) $\min _{q \in Q}\left(\boldsymbol{z}(q)=\left(\begin{array}{c}z_{1}(e) \\ \vdots \\ z_{k}(e)\end{array}\right)=\left(\begin{array}{c}\sum_{e \in q} c_{1}(e) \\ \vdots \\ \sum_{e \in q} c_{k}(e)\end{array}\right)\right)$

a multi-objective combinatorial optimization problem.

A solution that minimizes all objectives simultaneously does usually not exist. Therefore, we use the well-known concept of efficient solutions.

Notation 1. For two vectors $\boldsymbol{y}^{1}, \boldsymbol{y}^{2} \in \mathbb{R}^{k}$ we use the notation

$\boldsymbol{y}^{1} \leq \boldsymbol{y}^{2} \Leftrightarrow y_{i}^{1} \leqq y_{i}^{2}$ for $i=1, \ldots, k$ and $\boldsymbol{y}^{1} \neq \boldsymbol{y}^{2}$,

$\boldsymbol{y}^{1} \leqq \boldsymbol{y}^{2} \Leftrightarrow y_{i}^{1} \leqq y_{i}^{2}$ for $i=1, \ldots, k$.

In the following, we only use the symbols $<$ (strictly less than) and $\leqq$ (less than or equal to) to compare scalars.

Definition 2. A solution $q^{\prime} \in Q$ dominates another solution $q \in Q$ if $\boldsymbol{z}\left(q^{\prime}\right) \leq \boldsymbol{z}(q)$. We also say that $\boldsymbol{z}\left(q^{\prime}\right)$ dominates $\boldsymbol{z}(q)$. A solution $q \in Q$ is an efficient solution, if there is no $q^{\prime} \in Q$ such that $q^{\prime}$ dominates $q$. Then $\boldsymbol{z}(q)$ is called non-dominated.

Two efficient solutions $q, q^{\prime} \in Q$ are called equivalent if $\boldsymbol{z}(q)=$ $\boldsymbol{z}\left(q^{\prime}\right)$. A set of efficient solutions $\bar{Q} \subseteq Q$ is called complete if all $q \in$ $Q \backslash \bar{Q}$ are either dominated by or equivalent to at least one $q^{\prime} \in \bar{Q}$.

Solving (MOCO) means to find a complete set of efficient solutions.

We now assume that the input data is uncertain, i.e., the feasible set and/or the element costs $\boldsymbol{c}(e)$ are not exactly known in advance. If the set of feasible solutions is uncertain, we aim to find solutions which are feasible in all scenarios (as proposed in the seminal works on robustness, see, e.g., Ben-Tal, El Ghaoui, \& Nemirovski, 2009; Soyster, 1973). For this purpose, the sets of feasible solutions can be intersected in advance to obtain a (deterministic) set of robust feasible solutions. Hence, in the following, we assume the set $Q$ to be a deterministic set.
The uncertainty set $\mathcal{U}$ is then the set of all possible cost functions $\boldsymbol{c}$. The considered uncertainty set often strongly influences the solvability of uncertain optimization problems and the solution approaches. The idea of cardinality-constrained uncertainty is to assume that the parameters vary in intervals independent of each other, but not more than a given number of elements will be more expensive than their minimal cost. For example, there will not be an accident on every road of a transportation network at the same time, thus, a delay because of an accident does not need to be considered on all roads simultaneously. Bertsimas and Sim (2003) were the first to introduce cardinality-constrained uncertainty for single-objective uncertain combinatorial optimization problems. With $\hat{c}_{e}$ being the minimal or nominal value of $c(e)$ and $\hat{c}_{e}+\delta_{e}$ its maximal value, the considered uncertainty set can be written as

$\mathcal{U}^{c c}:=\left\{c: E \rightarrow \mathbb{R} \mid c(e) \in\left[\hat{c}_{e}, \hat{c}_{e}+\delta_{e}\right] \forall e \in E\right.$,

$$
\left.\left|\left\{e \in E \mid c(e)>\hat{c}_{e}\right\}\right| \leqq \Gamma\right\} .
$$

One possible extension to multi-objective optimization is to apply this approach to each objective independently (see Hassanzadeh et al., 2013):

Definition 3. For each element $e \in E$ and each objective $i$ let two real values $\hat{c}_{e, i}$ and $\delta_{e, i} \geqq 0$ be given. We assume that the uncertain cost $c_{i}(e)$ can take any value in the interval $\left[\hat{c}_{e, i}, \hat{c}_{e, i}+\delta_{e, i}\right]$, with $\hat{c}_{e, i}$ being the undisturbed value, called the nominal value. For each objective $i$ let an integer $\Gamma_{i} \leqq|E|$ be given. The cardinality-constrained uncertainty set contains all cost functions, with which for each objective $i$ at most $\Gamma_{i}$ elements differ from their nominal costs:

$$
\begin{aligned}
\mathcal{U}^{m c c} & :=\left\{\boldsymbol{c}: E \rightarrow \mathbb{R}^{k} \mid c_{i}(e) \in\left[\hat{c}_{e, i}, \hat{c}_{e, i}+\delta_{e, i}\right] \forall e \in E,\right. \\
\forall i & \left.=1, \ldots, k,\left|\left\{e \in E \mid c_{i}(e)>\hat{c}_{e, i}\right\}\right| \leqq \Gamma_{i} \forall i=1, \ldots, k\right\}
\end{aligned}
$$

We call the family of optimization problems

$$
\text { (MOUCO) }\left(\min _{q \in Q}\left(\boldsymbol{z}(q)=\sum_{e \in q} \boldsymbol{c}(e)\right), \boldsymbol{c} \in \mathcal{U}^{m c c}\right)
$$

a multi-objective uncertain combinatorial optimization problem with cardinality-constrained uncertainty. An instance of (MOUCO) is hence given by $(E, Q, \hat{C}, \Delta, \Gamma)$, with

$$
\begin{aligned}
\hat{C} & :=\left(\begin{array}{ccc}
\hat{c}_{e_{1}, 1} & \ldots & \hat{c}_{e_{1}, k} \\
\vdots & & \vdots \\
\hat{c}_{e_{|E|}, 1} & \ldots & \hat{c}_{e_{|E|}, k}
\end{array}\right), \Delta:=\left(\begin{array}{ccc}
\delta_{e_{1}, 1} & \ldots & \delta_{e_{1}, k} \\
\vdots & & \vdots \\
\delta_{e_{|E|}, 1} & \ldots & \delta_{e_{|E|}, k}
\end{array}\right), \\
\Gamma & :=\left(\Gamma_{1}, \ldots, \Gamma_{k}\right) .
\end{aligned}
$$

Note that with the uncertainty set $\mathcal{U}^{m c c}$, (MOUCO) is objective-wise uncertain, as it was defined by Ehrgott et al. (2014), i.e., the uncertainty sets in the objective functions are independent of each other.

This can usually be assumed, if the objectives are uncorrelated. However, also for correlated nominal values, the uncertainty can often be assumed to be uncorrelated, if unexpected events influence only one of the objectives.

To decide what is a good solution for a multi-objective uncertain problem is not trivial. In single-objective robust optimization one looks for so-called robust optimal solutions. Often these are defined as solutions, which have a minimal worst case value, i.e., one solves $\min _{q \in Q} \max _{c \in \mathcal{U}} z(q)$ (see, e.g., Ben-Tal et al., 2009). This concept has been generalized to robust efficiency for multiobjective problems in various ways (see, e.g., Ehrgott et al., 2014; Kuroiwa \& Lee, 2012). In this paper we determine the worst case independently for each objective (see Definition 4), as proposed by Kuroiwa and Lee (2012). This yields a single vector for each solution and these vectors can be compared using the methods of multi-objective optimization.

https://doi.org/10.1016/j.ejor.2017.12.018 


\section{A. Publications}

630

A. Raith et al./European Journal of Operational Research 267 (2018) 628-642

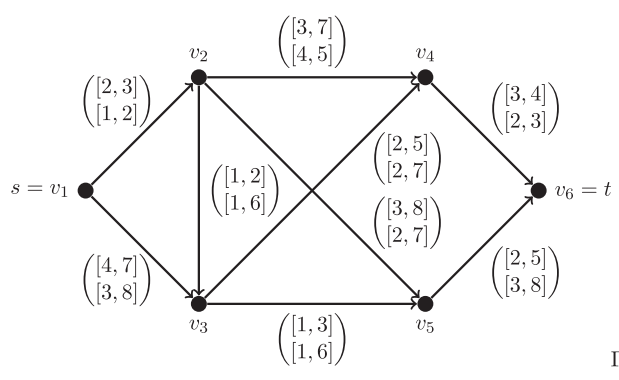

Fig. 1. An instance for (MOUSP).

Definition 4. A solution $q \in Q$ is robust efficient for (MOUCO) if $q$ is an efficient solution for the robust counterpart

(MORCO)

$$
\min _{q \in Q}\left(z^{\mathbf{R}}(q)=\left(\begin{array}{c}
\sup _{\boldsymbol{c} \in \mathcal{U}^{m c c}} z_{1}(q) \\
\vdots \\
\sup _{\boldsymbol{c} \in \mathcal{U}^{\text {mcc }}} z_{k}(q)
\end{array}\right)=\left(\begin{array}{c}
\sup _{\boldsymbol{c} \in \mathcal{U}^{\text {mcc }}} \sum_{e \in q} c_{1}(e) \\
\vdots \\
\sup _{\boldsymbol{c} \in \mathcal{U}^{\text {mcc }}} \sum_{e \in q} c_{k}(e)
\end{array}\right)\right) .
$$

Remark 5. Since (MOUCO) is objective-wise uncertain, robust efficiency, as defined in Definition 4, coincides with point-based and set-based minmax robust efficiency defined by Ehrgott et al (2014). Therefore, all results shown in this paper are valid for both concepts.

Analogously to Definition 2 we define:

Definition 6. Two robust efficient solutions $q, q^{\prime} \in Q$ are called equivalent if $\boldsymbol{z}^{\mathbf{R}}(q)=\boldsymbol{z}^{\mathbf{R}}\left(q^{\prime}\right)$. A set of robust efficient solutions $\bar{Q} \subseteq$ $Q$ is called complete if all $q \in Q \backslash \bar{Q}$ are either dominated w.r.t. $z^{\mathbf{R}}$ or equivalent to at least one $q^{\prime} \in \bar{Q}$.

\subsection{Example: a multi-objective uncertain shortest path problem}

Consider a graph $G=(V, E)$ with node set $V$ and edge set $E$, a start node $s \in V$ and a destination node $t \in V$. A path is a sequence of edges connecting adjacent nodes. In a simple path at most two edges are incident to each node. For a given cost function $c: E \rightarrow \mathbb{R}^{k}$ the cost of a path is obtained by following the path and adding up the costs of the edges traversed. Because simple paths do not contain any edge more than once, for a simple path $q$ we have $\boldsymbol{z}(q)=\sum_{e \in q} \boldsymbol{c}(e)$.

In the following, we assume conservative edge costs, i.e., every cycle $C$ has non-negative cost $z_{i}(C) \geqq 0$ for each cost function $\boldsymbol{c} \in \mathcal{U}^{m c c}$ and objective $i=1, \ldots, k$. Then, there always exists a complete set of robust efficient paths containing only simple paths. Hence, the multi-objective shortest path problem with cardinality-constrained uncertainty can be written as a combinatorial problem

(MOUSP) $\left(\min _{q \in Q} \sum_{e \in q} \boldsymbol{c}(e), \boldsymbol{c} \in \mathcal{U}^{m c c}\right)$

with $Q$ being the set of simple paths from $s$ to $t$ in $G$. We use the following example to illustrate the results and algorithms in this paper.

Example 7. Consider the network in Fig. 1 with $s=v_{1}$ and $t=v_{6}$ and $\Gamma_{1}=\Gamma_{2}=2$. The edge costs are given in the form

$\left(\begin{array}{l}{\left[\hat{c}_{e, 1}, \hat{c}_{e, 1}+\delta_{e, 1}\right]} \\ {\left[\hat{c}_{e, 2}, \hat{c}_{e, 2}+\delta_{e, 2}\right]}\end{array}\right)$
For this instance of (MOUSP), the set of robust efficient paths consists of the two paths

$q_{1}:=\left\{\left(v_{1}, v_{2}\right),\left(v_{2}, v_{4}\right),\left(v_{4}, v_{6}\right)\right\}$ with $z^{\mathbf{R}}\left(q_{1}\right)=\left(\begin{array}{c}13 \\ 9\end{array}\right)$,

$q_{2}:=\left\{\left(v_{1}, v_{2}\right),\left(v_{2}, v_{3}\right),\left(v_{3}, v_{5}\right),\left(v_{5}, v_{6}\right)\right\}$ with $z^{\mathbf{R}}\left(q_{2}\right)=\left(\begin{array}{l}11 \\ 16\end{array}\right)$

\section{Algorithms for finding robust efficient solutions in} multi-objective uncertain combinatorial optimization

We now consider (MOUCO), hence, we aim to find a complete set of efficient solutions for the robust counterpart (MORCO).

\subsection{Deterministic Subproblems Algorithm (DSA)}

The algorithms in this section are built upon an algorithm by Bertsimas and Sim (2003) for single-objective cardinalityconstrained uncertain combinatorial optimization problems, which we call Deterministic Subproblems Algorithm (DSA). Its idea is to find solutions for the uncertain problem by solving up to $|E|+1$ deterministic problems of the same type and comparing their solutions.

In Section 3.1.1, we first describe the algorithm by Bertsimas and $\operatorname{Sim}$ (2003) for single-objective problems. While the authors prove correctness of the algorithm with help of duality, we provide an alternative explanation, which we later extend to (MORCO). In Section 3.1.2, we extend the algorithm for the general multiobjective case and show that the number of subproblems can be further reduced for multi-objective problems with a special property. We present several ways to reduce the number of subproblems to be solved for both the single-objective and the multi-objective case.

\subsubsection{The DSA for single-objective problems}

We first consider the single-objective problem $\left(\min _{q \in Q} z(q), c \in\right.$ $\mathcal{U}^{c c}$ ) with $\mathcal{U}^{c c}$ defined as in Eq. (1).

We now explain the algorithm by Bertsimas and Sim (2003). A solution $q \in Q$ has maximal cost (we call this its worst case cost), if the costs of those $\Gamma$ elements, which have the largest cost intervals $\delta_{e}$ among all elements in $q$, take their maximal values $c(e)=\hat{c}_{e}+\delta_{e}$. If $q$ has fewer than $\Gamma$ elements, in the worst case the cost of all elements in $q$ take their maximal value.

Assume that the elements are ordered with respect to the interval length $\delta$, i.e.,

$\bar{\delta}_{1}:=\delta_{e_{1}} \geqq \bar{\delta}_{2}:=\delta_{e_{2}} \geqq \cdots \geqq \bar{\delta}_{|E|}:=\delta_{e_{|E|}} \geqq \bar{\delta}_{|E|+1}:=0$.

For each $l \in\{1, \ldots,|E|+1\}$ we define the function $g^{l}$ (see Bertsimas \& Sim, 2003):

$g^{l}(q):=\sum_{e \in q} \hat{c}_{e}+\Gamma \cdot \bar{\delta}_{l}+\sum_{\substack{e_{j} \in q \\ j \leqq l}}\left(\delta_{e_{j}}-\bar{\delta}_{l}\right)$

The function $g^{l}(q)$ is an approximation of the worst case costs of the set $q$. It contains

- the nominal cost $\hat{c}_{e}$ for each element $e \in q$, which has to be paid also in the worst case,

- $\bar{\delta}_{l} \cdot \Gamma$ since, in the worst case, the interval length $\delta_{e}$ has to be added to the costs for (at most) $\Gamma$ elements,

- the positive summand $\max \left\{0, \delta_{e}-\bar{\delta}_{l}\right\}$ for each element $e \in q$ to account for all elements in the set with higher interval lengths than $\bar{\delta}_{l}$.

The idea of the algorithm by Bertsimas and $\operatorname{Sim}$ (2003) is to solve all problems

$(\mathcal{P}(l)) \min _{q \in Q} g^{l}(q)$

https://doi.org/10.1016/j.ejor.2017.12.018 
for $l=1, \ldots|E|+1$ and compare the worst case values of all obtained solutions to choose a solution with minimal worst case cost. Instead of computing the worst case cost vectors, it is even sufficient to compare the objective values $g^{l}(q)$ of the obtained solutions and choose the solution with minimal objective value. This idea works due to the following two properties:

1. For every set $q$ and every $l \in\{1, \ldots,|E|+1\}$ we have that $g^{l}(q)$ is always greater than or equal to the worst case cost $z^{\mathrm{R}}(q)$.

2. For every set $q$ there exists some $l \in\{1, \ldots,|E|+1\}$ such that $g^{l}(q)$ equals the worst case cost $z^{\mathrm{R}}(q)$.

To show the first property, let $q$ be a set and let $\left\{e_{a_{1}}, \ldots, e_{a_{h}}\right\}$ be a subset of $h$ elements in $q$ with the largest cost intervals, where $h=\min \{|q|, \Gamma\}$. Then $z^{\mathrm{R}}(q)=\sum_{e \in q} \hat{c}_{e}+\sum_{j=1}^{h} \delta_{e_{a_{j}}}$ and we get

$$
g^{l}(q) \geqq \sum_{e \in q} \hat{c}_{e}+\sum_{j=1}^{h} \bar{\delta}_{l}+\sum_{j=1}^{h} \max \left\{0, \delta_{e_{a_{j}}}-\bar{\delta}_{l}\right\} \geqq z^{\mathrm{R}}(q) .
$$

For the second property we show that for each set $q$ there exists at least one index $l$ with $g^{l}(q)=z^{\mathrm{R}}(q)$ : If $q$ has less than $\Gamma$ elements, then

$g^{|E|+1}(q)=\sum_{e \in q} \hat{c}_{e}+\Gamma \cdot 0+\sum_{e \in q}\left(\delta_{e}-0\right)=z^{\mathrm{R}}(q)$.

If $q$ has at least $\Gamma$ elements, let $e_{\bar{l}}$ be the element in $q$ with the $\Gamma$ th smallest index. Then the $\Gamma$ elements $\left\{e_{j} \in q: j \leqq \bar{l}\right\}$ have the largest cost intervals in $q$ and it follows that

$$
\begin{aligned}
g^{\bar{l}}(q) & =\sum_{e \in q} \hat{c}_{e}+\Gamma \cdot \bar{\delta}_{\bar{l}}+\sum_{\substack{e_{j} \in q \\
j \leqq \bar{l}}}\left(\delta_{e_{j}}-\bar{\delta}_{\bar{l}}\right) \\
& =\sum_{e \in q} \hat{c}_{e}+\sum_{\substack{e_{j} \in q \\
j \leqq \bar{l}}} \bar{\delta}_{\bar{l}}+\sum_{\substack{e_{j} \in q \\
j \leqq \bar{l}}}\left(\delta_{e_{j}}-\bar{\delta}_{\bar{l}}\right)=z^{\mathrm{R}}(q) .
\end{aligned}
$$

Having these two properties, we see that a robust optimal solution $q^{*}$ is optimal for the problem $(\mathcal{P}(\bar{l}))$, since none of the other sets $q \in Q$ can have a better objective value. Therefore, at least one robust optimal solution will be found by the algorithm.

Algorithm 1 shows the basic structure of the described algo-

Algorithm 1 Basic structure of DSA (based on Bertsimas \& Sim, 2003).

Input: an instance $I=(E, Q, \hat{\boldsymbol{c}}, \boldsymbol{\delta}, \Gamma)$ of (MOUCO) with $k=1$

Output: a robust efficient solution for $I$

1: Sort $E$ w.r.t. $\delta_{e}$ such that $\bar{\delta}_{1}:=\delta_{e_{1}} \geqq \bar{\delta}_{2}:=\delta_{e_{2}} \geqq \cdots \geqq \bar{\delta}_{|E|} \geqq$ $\bar{\delta}_{|E|+1}:=0$.

2: Set $L:=\{1, \ldots,|E|+1\}$.

3: For all $l \in L$ find an optimal solution $q^{l}$ for $(\mathcal{P}(l))$.

4: Compare the objective values $z^{\mathrm{R}}\left(q^{l}\right)$ for all $l \in L$. The solution with the smallest objective value is a robust optimal solution.

rithm. First, the elements are ordered with respect to their interval lengths. Then the subproblems defined above are solved. Finally, of all obtained solutions the one with minimal objective value w.r.t. the respective subproblem is chosen.

The efficiency of Algorithm 1 depends on the time complexity to solve the subproblems $(\mathcal{P}(l))$. Because the summand $\Gamma \cdot \bar{\delta}_{l}$ is solution-independent, a solution for $(\mathcal{P}(l))$ can be found efficiently by solving a problem of the same kind as the underlying deterministic problem with element costs

$c^{l}\left(e_{j}\right):= \begin{cases}\hat{c}_{e_{j}}+\left(\delta_{e_{j}}-\bar{\delta}_{l}\right) & \text { for } j<l \\ \hat{c}_{e_{j}} & \text { for } j \geqq l .\end{cases}$

Hence, Algorithm 1 finds a robust optimal solution in polynomial time for many combinatorial optimization problems. Examples are the minimum spanning tree and the shortest path problem.
In the following, we show how Algorithm 1 can be enhanced. It is not necessary to solve all of the $|E|+1$ subproblems introduced above. The following three results (see Bertsimas \& Sim, 2003; Lee \& Kwon, 2014; Park \& Lee, 2007) can be used to reduce the number of subproblems (Lemma 8): First, if two elements have the same interval length $\delta_{e}$, then their associated subproblems are identical. Second, the worst case cost of a set $q$ with at least $\Gamma$ elements equals its objective value $g^{l}(q)$ not only for one subproblem, but for two consecutive subproblems. Therefore, we do not miss any solutions if we only solve every second problem. Third, none of the first $\Gamma-1$ elements can be the one with the $\Gamma$ th smallest index for any set in $Q$, so their associated subproblems need not to be solved.

Lemma 8. (Bertsimas and Sim, 2003; Lee and Kwon, 2014; Park and Lee, 2007). The number of subproblems to be solved by Algorithm 1 can be reduced to at most $\left\lceil\frac{|E|-\Gamma}{2}\right\rceil+1$ in the following ways:

1. If there are several elements $e_{1}, \ldots, e_{(l+h)}$ with the same interval length $\delta_{e_{1}}=\cdots=\delta_{e_{1+h}}$, only one of the subproblems $\mathcal{P}(l), \ldots, \mathcal{P}(l+h)$ needs to be solved (Bertsimas \& Sim, 2003).

2. Only every second subproblem and the last subproblem need to be solved (Lee E' Kwon, 2014).

3. It is sufficient to start with the $\Gamma$ th subproblem (Park E Lee, 2007).

Depending on the solutions that are found while the algorithm is executed, we can further reduce the number of subproblems to be solved. We refer to this newly proposed enhancement as solution checking.

Lemma 9. Let $1 \leqq \tilde{l}<l \leqq|E|+1$ and let $q^{\tilde{I}}$ be an optimal solution for $\mathcal{P}(\tilde{l})$. If $q^{\tilde{l}}$ does not contain any of the elements $e_{1}, \ldots, e_{l-1}$, then it is optimal for $\mathcal{P}(l)$.

Proof. We can find a solution of $\mathcal{P}(l)$ by solving a problem with the deterministic costs given in (3). For these costs we have

$$
\begin{aligned}
\tilde{l} \leqq l & \Rightarrow \bar{\delta}_{\tilde{l}} \geqq \bar{\delta}_{l} \Rightarrow c^{\tilde{l}}\left(e_{j}\right) \leqq c^{l}\left(e_{j}\right) \forall e_{j}: j<\tilde{l}, \\
j \leqq l & \Rightarrow \delta_{e_{j}} \geqq \bar{\delta}_{l} \\
& \Rightarrow c^{\tilde{l}}\left(e_{j}\right)=\hat{c}_{e_{j}} \leqq \hat{c}_{e_{j}}+\left(\delta_{e_{j}}-\bar{\delta}_{l}\right)=c^{l}\left(e_{j}\right) \forall e_{j}: \tilde{l} \leqq j<l, \\
\tilde{l} \leqq l & \Rightarrow c^{\tilde{l}}\left(e_{j}\right)=\hat{c}_{e_{j}}=c^{l}\left(e_{j}\right) \forall e_{j}: j \geqq l .
\end{aligned}
$$

If $q^{\tilde{l}}$ does not contain any element $e_{j}: j<l$, then

$$
\sum_{e \in q^{\tilde{I}}} c^{l}(e)=\sum_{e \in q^{\tilde{I}}} c^{\tilde{l}}(e) \leqq \sum_{e \in q} c^{\tilde{l}}(e) \leqq \sum_{e \in q} c^{l}(e) \forall q \in Q,
$$

hence, $q^{\tilde{l}}$ is optimal for $\mathcal{P}(l)$. $\square$

We can therefore replace Step 3 of the basic structure (Algorithm 1) with Algorithm 2.

Algorithm 2 Improved Step 3 of Algorithm 1: solve subproblems (with solution checking).

Input: $I=(E, Q, \hat{\boldsymbol{c}}, \boldsymbol{\delta}, \Gamma)$ with $E$ ordered w.r.t. $\boldsymbol{\delta}, \overline{\boldsymbol{\delta}}$, an index set $L$ of subproblems

Output: a set of solutions $\left\{q^{l}: l \in L\right\}$

1: $\tilde{l}:=0$

2: for all $l \in L$ in increasing order do

if $\tilde{l}=0$ or $q^{\tilde{l}}$ contains any element in $\left\{e_{1}, \ldots, e_{l-1}\right\}$ then

Find an optimal solution $q^{l}$ for $(\mathcal{P}(l))$.

else $q^{l}:=q^{\tilde{l}}$

end if

$\tilde{l}:=l$

end for

https://doi.org/10.1016/j.ejor.2017.12.018 
Lemma 9 does not contain any theoretical complexity result since, in the worst case, still $\left\lceil\frac{|E|-\Gamma}{2}\right\rceil+1$ subproblems are solved. Nevertheless, the results of our experiments in Section 4 show the practical use of this improvement.

\subsubsection{The DSA for multi-objective problems}

In this section, we extend the DSA to multi-objective problems. The idea presented in Section 3.1.1 is still valid. A set $q$ has maximal cost in the $i$ th objective, if the cost of its $\Gamma_{i}$ elements with the largest cost intervals $\delta_{e, i}$ take their maximal value. However, the sorting of the elements by interval lengths often results in a different order for each objective. An element that has the $\Gamma_{i}$ th longest interval in $q$ for all $i=1, \ldots, k$ is not likely to exist. To ensure that the worst case vector of $q$ equals the objective vector of a subproblem, we have to iterate through all elements for each objective independently and consider all possible combinations. The subproblems to be solved are hence constructed in the following way:

For $j=1, \ldots,|E|, i=1, \ldots, k$ let $E_{j}^{i}$ be a set of the $j$ elements with the largest intervals for the $i$ th objective with $E_{1}^{i} \subset E_{2}^{i} \subset$ $\cdots \subset E_{|E|}^{i}=E$. I.e., $\left|E_{j}^{i}\right|=j$ and $\delta_{e, i} \geqq \delta_{e^{\prime}, i} \forall e \in E_{j}^{i}, e^{\prime} \in E \backslash E_{j}^{i}$. We further define $\bar{\delta}_{j}^{i}:=\min _{e \in E_{j}^{i}} \delta_{e, i}$ and $\bar{\delta}_{|E|+1}^{i}:=0 \forall i$. For each $l=\left(l_{1}, \ldots, l_{k}\right) \in L:=\{1, \ldots,|E|+1\} \times \cdots \times\{1, \ldots,|E|+1\} \quad$ we define the problem

$(\mathcal{M P}(\boldsymbol{l}))$

$$
\min _{q \in Q} \boldsymbol{g}^{\mathbf{l}}(q):=\left(\begin{array}{c}
\sum_{e \in q} \hat{e}_{e, 1}+\Gamma_{1} \cdot \bar{\delta}_{l_{1}}^{1}+\sum_{e \in q \cap E_{l_{1}}^{1}}\left(\delta_{e, 1}-\bar{\delta}_{l_{1}}^{1}\right) \\
\vdots \\
\sum_{e \in q} \hat{c}_{e, k}+\Gamma_{k} \cdot \bar{\delta}_{l_{k}}^{k}+\sum_{e \in q \cap E_{l_{k}}^{k}}\left(\delta_{e, k}-\bar{\delta}_{l_{k}}^{k}\right)
\end{array}\right) .
$$

We are now looking for a complete set of solutions for each of the subproblems. Such a solution set can be found by solving a deterministic multi-objective problem of the same kind as the original problem. We denote the solution set that we obtain for $\mathcal{M P}(\boldsymbol{l})$ by $O P T^{l}$.

Algorithm 3 preserves the basic structure of DSA: first, the

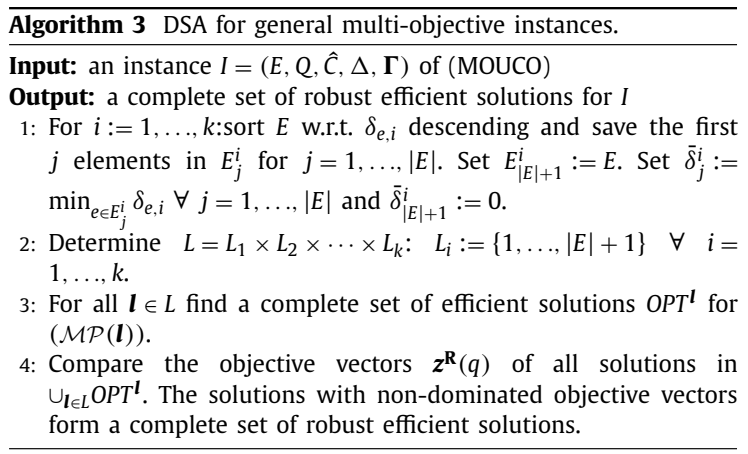

elements are sorted w.r.t. $\delta_{e, i}$ for each $i=1, \ldots, k$. Instead of changing the indices, we store the set $E_{j}^{i}$ of the first $j$ elements for all $j=1, \ldots,|E|$, because the order of the elements depends on the objective. Then the set $L$ is determined, which contains vectors instead of scalar values. For each element in $L$ the subproblem defined above is solved and their solutions are compared to obtain the robust efficient solutions.

Theorem 10. Algorithm 3 finds a complete set of robust efficient solutions for (MOUCO).
Proof. First, we show that $\mathbf{g}^{\mathbf{l}}$ never underestimates $\boldsymbol{z}^{\mathbf{R}}$ for any objective. Further, we prove that for each feasible solution $q$ there is an $\boldsymbol{l} \in L$ with $\boldsymbol{g}^{\boldsymbol{l}}(q)=\boldsymbol{z}^{\mathbf{R}}(q)$. We conclude that Algorithm 3 finds a complete set of robust efficient solutions.

For each $q \in Q, \boldsymbol{l} \in L$ and $i \in\{1, \ldots, k\}$ we show $z_{i}^{\mathrm{R}}(q) \leqq g_{i}^{l}(q)$. Let $\left\{e_{a_{1}}, \ldots, e_{a_{h}}\right\}$ be a set of $h$ elements in $q$ with the largest cost intervals $\delta_{e, i}$, where $h=\min \left\{|q|, \Gamma_{i}\right\}$. Then

$$
\begin{aligned}
z_{i}^{\mathrm{R}}(q)= & \sum_{e \in q} \hat{c}_{e, i}+\sum_{j=1}^{h}\left(\delta_{e_{a_{j}}, i}-\bar{\delta}_{l_{i}}^{i}+\bar{\delta}_{l_{i}}^{i}\right) \\
\leqq & \sum_{e \in q} \hat{c}_{e, i}+\Gamma_{i} \cdot \bar{\delta}_{l_{i}}^{i}+\sum_{j=1}^{h}\left(\delta_{e_{a_{j}}, i}-\bar{\delta}_{l_{i}}^{i}\right) \text { since } h \leqq \Gamma_{i} \\
\leqq & \sum_{e \in q} \hat{c}_{e, i}+\Gamma_{i} \cdot \bar{\delta}_{l_{i}}^{i}+\sum_{e \in q} \max \left\{0, \delta_{e, i}-\bar{\delta}_{l_{i}}^{i}\right\} \\
& \text { since }\left\{e_{a_{1}}, \ldots, e_{a_{h}}\right\} \subseteq q \\
= & \sum_{e \in q} \hat{c}_{e, i}+\Gamma_{i} \cdot \bar{\delta}_{l_{i}}^{i}+\sum_{e \in q \cap E_{l_{i}}^{i}}\left(\delta_{e, i}-\bar{\delta}_{l_{i}}^{i}\right) \\
& \text { since } e \in E_{l_{i}}^{i} \Rightarrow \delta_{e, i} \geqq \bar{\delta}_{l_{i}}^{i}, e \notin E_{l_{i}}^{i} \Rightarrow \delta_{e, i} \leqq \bar{\delta}_{l_{i}}^{i} \\
= & g_{i}^{l}(q) .
\end{aligned}
$$

We conclude $\boldsymbol{g}^{\boldsymbol{l}}(q) \leqq \boldsymbol{z}^{\mathbf{R}}(q)$ for all $q \in Q$ and $\boldsymbol{l} \in L$.

We show now that for every $q \in Q$ there is an $\overline{\boldsymbol{l}} \in L$ with $\boldsymbol{g}^{\bar{l}}(q)=\boldsymbol{z}^{\mathbf{R}}(q)$. Given $q \in Q$ we construct $\overline{\boldsymbol{l}}$ as follows: For all $i \in\{1, \ldots, k\}$ with $\Gamma_{i}>|q|$, we set $\bar{l}_{i}:=|E|+1$, since

$z_{i}^{\mathrm{R}}(q)=\sum_{e \in q} \hat{c}_{e, i}+\sum_{e \in q} \delta_{e, i}=\sum_{e \in q} \hat{c}_{e, i}+\Gamma_{i} \cdot 0+\sum_{e \in q}\left(\delta_{e, i}-0\right)$.

For all $i \in\{1, \ldots, k\}$ with $\Gamma_{i} \leqq|q|$ we choose $\bar{l}_{i}$ such that $q \cap E_{\bar{l}_{i}}^{i}$ contains exactly $\Gamma_{i}$ elements. These $\Gamma_{i}$ elements have the largest cost intervals $\delta_{e, i}$ among all elements in $q$, i.e., the worst case cost for $q$ is

$$
\begin{aligned}
z_{i}^{\mathrm{R}}(q) & =\sum_{e \in q} \hat{c}_{e, i}+\sum_{e \in q \cap E_{\bar{l}_{i}}^{i}} \delta_{e, i} \\
& =\sum_{e \in q} \hat{c}_{e, i}+\sum_{e \in q \cap E_{\bar{l}_{i}}^{i}} \bar{\delta}_{\bar{l}_{i}}^{i}+\sum_{e \in q \cap E_{\bar{l}_{i}}^{i}}\left(\delta_{e, i}-\bar{\delta}_{\bar{l}_{i}}^{i}\right) \\
& =\sum_{e \in q} \hat{c}_{e, i}+\Gamma_{i} \cdot \bar{\delta}_{\bar{l}_{i}}^{i}+\sum_{e \in q \cap E_{\bar{l}_{i}}^{i}}\left(\delta_{e, i}-\bar{\delta}_{\bar{l}_{i}}^{i}\right) \text { since }\left|q \cap E_{\bar{l}_{i}}^{i}\right|=\Gamma_{i} .
\end{aligned}
$$

We conclude $\boldsymbol{z}^{\mathbf{R}}(q)=\boldsymbol{g}^{\bar{I}}(q)$. If $q$ is robust efficient, then there is no $q^{\prime} \in Q$ with $\boldsymbol{z}^{\mathbf{R}}\left(q^{\prime}\right) \leq \boldsymbol{z}^{\mathbf{R}}(q)$. It follows that

$\nexists q^{\prime} \in Q: z^{\mathbf{R}}\left(q^{\prime}\right) \leqq z^{\mathbf{R}}(q) \stackrel{z^{\mathbf{R}}\left(q^{\prime}\right) \leqq \boldsymbol{g}^{\bar{I}}\left(q^{\prime}\right)}{\Rightarrow}$

$\nexists q^{\prime} \in Q: \boldsymbol{g}^{\bar{I}}\left(q^{\prime}\right) \leqq \boldsymbol{z}^{\mathbf{R}}(q)=\mathbf{g}^{\bar{I}}(q)$.

Therefore, $q$ or an equivalent solution is found at least once in the algorithm. It follows that in Step 4 the objective vector of each found solution is compared to all non-dominated objective vectors, thus only robust efficient solutions remain. It follows that the output is a complete set of robust efficient solutions.

Example 11. Consider the instance in Example 7 (Fig. 1). In Step 1 of Algorithm 3 we obtain

$\bar{\delta}^{1}=(5,4,3,3,3,2,1,1,1)^{T}, \bar{\delta}^{2}=(5,5,5,5,5,5,1,1,1)^{T}$

and for example

$E_{1}^{1}=\left\{\left(v_{2}, v_{5}\right)\right\}, E_{2}^{1}=E_{1}^{1} \cup\left\{\left(v_{2}, v_{4}\right)\right\}, E_{3}^{1}=E_{2}^{1} \cup\left\{\left(v_{1}, v_{3}\right)\right\}$,

$E_{4}^{1}=E_{2}^{1} \cup\left\{\left(v_{3}, v_{4}\right)\right\}, E_{5}^{1}=E_{4}^{1} \cup\left\{\left(v_{5}, v_{6}\right)\right\}, E_{6}^{1}=E_{5}^{1} \cup\left\{\left(v_{3}, v_{5}\right)\right\}$,

$E_{7}^{1}=E_{6}^{1} \cup\left\{\left(v_{1}, v_{2}\right)\right\}, E_{8}^{1}=E_{7}^{1} \cup\left\{\left(v_{2}, v_{3}\right)\right\}, E_{9}^{1}=E_{8}^{1} \cup\left\{\left(v_{4}, v_{6}\right)\right\}$,

$E_{1}^{2}=\left\{\left(v_{2}, v_{5}\right)\right\}, E_{2}^{2}=E_{1}^{2} \cup\left\{\left(v_{2}, v_{4}\right)\right\}, E_{3}^{2}=E_{2}^{2} \cup\left\{\left(v_{1}, v_{3}\right)\right\}$,

https://doi.org/10.1016/j.ejor.2017.12.018 


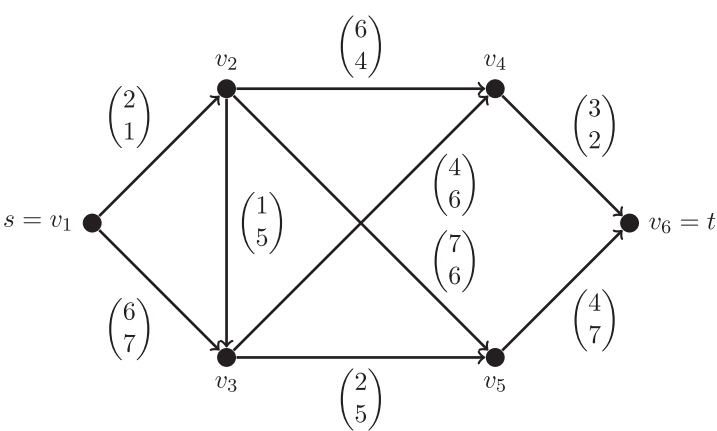

Fig. 2. $O P T^{(7,8)}$ in Example 11 is obtained by solving this instance of the multiobjective shortest path problem.

$E_{4}^{2}=E_{2}^{2} \cup\left\{\left(v_{3}, v_{4}\right)\right\}, E_{5}^{2}=E_{4}^{2} \cup\left\{\left(v_{5}, v_{6}\right)\right\}, E_{6}^{2}=E_{5}^{2} \cup\left\{\left(v_{3}, v_{5}\right)\right\}$, $E_{7}^{2}=E_{6}^{2} \cup\left\{\left(v_{1}, v_{2}\right)\right\}, E_{8}^{2}=E_{7}^{2} \cup\left\{\left(v_{2}, v_{3}\right)\right\}, E_{9}^{2}=E_{8}^{2} \cup\left\{\left(v_{4}, v_{6}\right)\right\}$. Step 3 sets $L:=\{1, \ldots, 9\} \times\{1, \ldots, 9\}$ and in Step $3(\mathcal{M P}(\boldsymbol{I}))$ is solved for all $\boldsymbol{l} \in L$.

As an example, we consider $\boldsymbol{l}=(7,8)$. Recall, that the path $q_{1}:=\left(\left(v_{1}, v_{2}\right),\left(v_{2}, v_{4}\right),\left(v_{4}, v_{6}\right)\right)$ is robust efficient. Since $\left|E_{7}^{1} \cap q_{1}\right|=2$ and $\left|E_{8}^{2} \cap q_{1}\right|=2$, we know from the proof of Theorem 10 that $\mathbf{g}^{\mathbf{( 7 , 8 )}}\left(q_{1}\right)=\boldsymbol{z}^{\mathbf{R}}\left(q_{1}\right)$ and that $q_{1}$ is an efficient solution for

$(\mathcal{M P}(7,8))$

$$
\min _{q \in Q} \mathbf{g}^{(\mathbf{7}, \boldsymbol{8})}(q):=\left(\begin{array}{l}
\sum_{e \in q} \hat{c}_{e, 1}+\Gamma_{1} \cdot \bar{\delta}_{7}^{1}+\sum_{e \in q \cap E_{7}^{7}}\left(\delta_{e, 1}-\bar{\delta}_{7}^{1}\right) \\
\sum_{e \in q} \hat{e}_{e, 2}+\Gamma_{2} \cdot \bar{\delta}_{8}^{2}+\sum_{e \in q \cap E_{8}^{2}}\left(\delta_{e, 2}-\bar{\delta}_{8}^{2}\right)
\end{array}\right) .
$$

A complete set of efficient solutions $O P T^{(7,8)}$ for $(\mathcal{M P}(7,8))$ can be obtained by solving the instance of the deterministic multiobjective shortest path problem shown in Fig. 2. The edge costs are

$c_{1}^{(7,8)}(e):= \begin{cases}\hat{c}_{e, 1}+\delta_{e, 1}-\bar{\delta}_{7}^{1} & \text { if } e \in E_{7}^{1} \\ \hat{c}_{e, 1} & \text { else }\end{cases}$

$c_{2}^{(7,8)}(e):= \begin{cases}\hat{c}_{e, 2}+\delta_{e, 2}-\bar{\delta}_{8}^{2} & \text { if } e \in E_{8}^{2} \\ \hat{c}_{e, 2} & \text { else. }\end{cases}$

The path $q_{1}$ is indeed efficient for this instance with $\boldsymbol{c}^{(\mathbf{7}, 8)}\left(q_{1}\right)=(11,7)^{T}$. It follows

$\mathbf{g}^{(\mathbf{7 , 8 )}}\left(q_{1}\right)=\left(\begin{array}{c}\Gamma_{1} \cdot \bar{\delta}_{7}^{1}+11 \\ \Gamma_{2} \cdot \bar{\delta}_{8}^{2}+7\end{array}\right)=\left(\begin{array}{c}13 \\ 9\end{array}\right)=\boldsymbol{z}^{\mathbf{R}}\left(q_{1}\right)$.

The path $q_{3}:=\left\{\left(v_{1}, v_{2}\right),\left(v_{2}, v_{3}\right),\left(v_{3}, v_{4}\right),\left(v_{4}, v_{6}\right)\right\}$ is efficient for this instance as well, hence $q_{1}, q_{3} \in O P T^{7,8)}$.

In Step 4 of Algorithm 3, all obtained solutions are compared to each other. The path $q_{3}$ is not robust efficient, because $\boldsymbol{z}^{\mathbf{R}}\left(q_{2}\right)=(11,16)^{T} \leq(12,16)^{T}=\boldsymbol{z}^{\mathbf{R}}\left(q_{3}\right)$. Since $q_{2} \in O P T^{(4,4)}, \boldsymbol{z}^{\mathbf{R}}\left(q_{2}\right)$ and $\boldsymbol{z}^{\mathbf{R}}\left(q_{3}\right)$ are compared to each other in Step 4 and the returned solution set does not contain $q_{3}$. However, it contains $q_{1}$, because $q_{1}$ is robust efficient and hence there does not exist any path $q^{\prime}$ with $\boldsymbol{z}^{\mathbf{R}}\left(q^{\prime}\right) \leq \boldsymbol{z}^{\mathbf{R}}\left(q_{1}\right)$.

As for the single-objective version, we can reduce the number of subproblems to be solved. The results of Lemma 8 are still valid for each objective independently. Therefore, we can replace the $L_{i}$ as described in the following lemma.

Lemma 12. The number of subproblems to be solved by Algorithm 3 can be reduced to $\prod_{i=1}^{k}\left(\left\lceil\frac{|E|-\Gamma_{i}}{2}\right\rceil+1\right)$ in the same ways as in the single-objective case (Lemma 8):
1. Let $i \in\{1, \ldots, k\}$ be given. If there are several elements with the same interval length $\delta_{e, i}$, i.e., there exist pairwise different indices $j_{1}, \ldots, j_{h} \in\{1, \ldots,|E|\}$ with $\bar{\delta}_{j_{1}}^{i}=\cdots=\bar{\delta}_{j_{h}}^{i}$, then it is sufficient that $l_{i}$ takes one of the values in $\left\{j_{1}, \ldots, j_{h}\right\}$.

2. For all $i \in\{1, \ldots, k\}$ it is sufficient, that $l_{i}$ takes every second value in $\{1, \ldots,|E|\}$ and the value $|E|+1$.

3. It is sufficient that $l_{i}$ takes values that are greater than or equal to $\Gamma_{i}$.

Proof.

1. Let $\hat{l}_{1}, \hat{l}_{2}, \ldots, \hat{l}_{i-1}, \hat{l}_{i+1}, \ldots, \hat{l}_{k} \in\{1, \ldots,|E|+1\}$ be fixed values. We define the vector $\hat{\boldsymbol{l}}^{x}:=\left(\hat{l}_{1}, \hat{l}_{2}, \ldots, \hat{l}_{i-1}, x, \hat{l}_{i+1}, \ldots, \hat{l}_{k}\right)$. From $\bar{\delta}_{j_{1}}^{i}=\cdots=\bar{\delta}_{j_{h}}^{i}$ it follows directly

$$
\begin{aligned}
& \sum_{e \in q} \hat{c}_{e, i}+\Gamma_{i} \cdot \bar{\delta}_{j_{1}}^{i}+\sum_{e \in q \cap E_{j_{1}}^{i}}\left(\delta_{e, i}-\bar{\delta}_{j_{1}}^{i}\right) \\
& =\cdots=\sum_{e \in q} \hat{c}_{e, i}+\Gamma_{i} \cdot \bar{\delta}_{j_{h}}^{i}+\sum_{e \in q \cap E_{j_{h}}^{i}}\left(\delta_{e, i}-\bar{\delta}_{j_{h}}^{i}\right)
\end{aligned}
$$

and therefore $\mathcal{M P}\left(\hat{\boldsymbol{I}}_{\mathbf{1}}\right)=\cdots=\mathcal{M P}\left(\hat{\boldsymbol{l}}_{\boldsymbol{h}}\right)$.

2. Let $q \in Q$ be a feasible solution. We have shown in the proof of Theorem 10 that there exists an $\overline{\boldsymbol{i}} \in L$ with $\boldsymbol{z}^{\mathbf{R}}(q)=\boldsymbol{g}^{\bar{I}}(q)$ and either $\bar{l}_{i}=|E|+1$ or $\Gamma_{i}=\left|q \cap E_{\bar{l}_{i}}^{i}\right|$. In the second case, since $q \cap$ $E_{i_{i}}^{i}$ contains the $\Gamma_{i}$ elements in $q$ with the largest cost intervals $\delta_{e, i}$, we have

$$
\begin{aligned}
z_{i}^{\mathrm{R}}(q)= & \sum_{e \in q} \hat{c}_{e, i}+\Gamma_{i} \cdot \bar{\delta}_{\bar{l}_{i}}^{i}+\sum_{e \in q \cap E_{\bar{l}_{i}}^{i}}\left(\delta_{e, i}-\bar{\delta}_{\bar{l}_{i}}^{i}\right) \\
= & \sum_{e \in q} \hat{c}_{e, i}+\Gamma_{i} \cdot \bar{\delta}_{\bar{l}_{i}}^{i}+\sum_{e \in q \cap E_{\bar{l}_{i}}^{i}}\left(\delta_{e, i}-\bar{\delta}_{\bar{l}_{i}}^{i}\right)+\Gamma_{i} \cdot\left(\bar{\delta}_{\left(\bar{l}_{i}+1\right)}^{i}-\bar{\delta}_{\bar{l}_{i}}^{i}\right) \\
& +\Gamma_{i} \cdot\left(\bar{\delta}_{\bar{l}_{i}}^{i}-\bar{\delta}_{\left(\bar{l}_{i}+1\right)}^{i}\right) \\
= & \sum_{e \in q} \hat{c}_{e, i}+\Gamma_{i} \cdot \bar{\delta}_{\left(\bar{l}_{i}+1\right)}^{i}+\sum_{e \in q \cap E_{l_{i}}^{i}}\left(\delta_{e, i}-\bar{\delta}_{\left(\bar{l}_{i}+1\right)}^{i}\right), \\
& \text { because }\left|q \cap E_{l_{i}}^{i}\right|=\Gamma_{i} \\
= & \sum_{e \in q} \hat{c}_{e, i}+\Gamma_{i} \cdot \bar{\delta}_{\left(\bar{l}_{i}+1\right)}^{i}+\sum_{e \in q \cap E_{\left(\bar{l}_{i}+1\right)}^{i}}\left(\delta_{e, i}-\bar{\delta}_{\left(\bar{l}_{i}+1\right)}^{i}\right),
\end{aligned}
$$

because $\delta_{e, i}=\bar{\delta}_{\left(\bar{l}_{i}+1\right)}^{i}$ for $e \in E_{\left(\bar{l}_{i}+1\right)}^{i} \backslash E_{\bar{l}_{i}}^{i}$. Therefore, if $\Gamma_{i} \leqq|q|$, it is sufficient that $l_{i}$ either takes the value $\bar{l}_{i}$ or $\bar{l}_{i}+1$. If $\Gamma_{i} \geqq|q|$, it is sufficient that $l_{i}$ takes the value $|E|+1$.

3. In the proof of Theorem 10 we have show that for every $q \in Q$ there is an $\overline{\boldsymbol{l}} \in L$ with $\boldsymbol{z}^{\mathbf{R}}(q)=\boldsymbol{g}^{\overline{\boldsymbol{I}}}(q)$ and either $\bar{l}_{i}=|E|+1$ or $\Gamma_{i}=\left|q \cap E_{\bar{l}_{i}}^{i}\right| \leqq\left|E_{\bar{l}_{i}}^{i}\right|=\bar{l}_{i}$.

From statement 3 we know that $l_{i}$ takes at most $|E|+1-\left(\Gamma_{i}-1\right)$ different values. From statement 2 it follows that of these values the last one and every second of the other ones are sufficient. This leads to at most

$$
\begin{aligned}
\left\lfloor\frac{|E|+1-\left(\Gamma_{i}-1\right)-1}{2}\right\rfloor+1 & =\left\lfloor\frac{|E|-\Gamma_{i}+1}{2}\right\rfloor+1 \\
& =\left\lceil\frac{|E|-\Gamma_{i}}{2}\right\rceil+1
\end{aligned}
$$

different values of $l_{i}$. Therefore, it is sufficient to solve $\prod_{i=1}^{k}\left(\left\lceil\frac{|E|-\Gamma_{i}}{2}\right\rceil+1\right)$ subproblems.

Here again, we can use solution checking, i.e., skip some additional subproblems, depending on the solutions found so far. However, we now have to ensure that $\tilde{\boldsymbol{l}} \leq \boldsymbol{l}$ and that none of the

https://doi.org/10.1016/j.ejor.2017.12.018 
solutions in $O P T^{\tilde{l}}$ contains any of the elements, whose costs have been increased.

Lemma 13. Let $\boldsymbol{l}, \tilde{\boldsymbol{l}} \in \mathbb{Z}^{k}$ be given with $\tilde{\boldsymbol{l}} \leq \boldsymbol{l}$ and let $J$ be the set of indices $i$ with $\tilde{l}_{i}<l_{i}$. Let OPT $\tilde{I}$ be a complete set of efficient solutions for $\mathcal{M P}(\tilde{\boldsymbol{l}})$. If none of the sets in $O P T^{\tilde{l}}$ contains an element in $\cup_{i \in J} E_{l_{i}}^{i}$, then $O P T^{\tilde{I}}$ is a complete set of efficient solutions for $\mathcal{M P}(\boldsymbol{l})$.

Proof. Since $\Gamma_{i} \cdot \bar{\delta}_{i}^{l_{i}}$ are solution independent constants, the minimization problem to be solved is a deterministic multi-objective problem with costs $\boldsymbol{c}^{\boldsymbol{l}}(e)=\left(c_{1}^{\boldsymbol{l}}(e), \ldots, c_{k}^{\boldsymbol{l}}(e)\right)$ :

$c_{i}^{l}(e):= \begin{cases}\hat{c}_{e, i}+\left(\delta_{e, i}-\bar{\delta}_{l_{i}}^{i}\right) & \text { for } e \in E_{l_{i}}^{i} \\ \hat{c}_{e, i} & \text { else. }\end{cases}$

Since $\tilde{l}_{i} \leqq l_{i} \Rightarrow \bar{\delta}_{\tilde{l}}^{i} \geqq \bar{\delta}_{l}^{i}$, it follows

$c_{i}^{\tilde{l}}(e)=c_{i}^{l}(e) \forall i$ with $l_{i}=\tilde{l}_{i}, \forall e \in E$

$c_{i}^{\tilde{l}}(e)=c_{i}^{l}(e) \forall i$ with $\tilde{l}_{i}<l_{i}, \forall e \in E \backslash E_{l_{i}}^{i}$

$c_{i}^{\tilde{I}}(e) \leqq c_{i}^{l}(e) \forall i, \forall e \in E$.

Hence, if none of the sets in $O P T^{\tilde{l}}$ contains any element in $\cup_{i \in J} E_{l_{i}}^{i}$, we have $c_{i}^{\tilde{l}}(e)=c_{i}^{l}(e)$ for all elements that are contained in any set in $O P T^{\tilde{I}}$, and $\tilde{c}_{i}^{\tilde{I}}(e) \leqq c_{i}^{l}(e)$ for all elements in $E$. It follows, that every $q \in O P T^{\tilde{l}}$ is also efficient w.r.t $\boldsymbol{c}^{l}$. Furthermore, for every $q^{\prime} \notin$ $O P T^{\tilde{l}}$ exists a $q \in O P T^{\tilde{l}}$ with

$\sum_{e \in q} \boldsymbol{c}^{\boldsymbol{l}}(e)=\sum_{e \in q} \boldsymbol{c}^{\tilde{l}}(e) \leqq \sum_{e \in q^{\prime}} \boldsymbol{c}^{\tilde{l}}(e) \leqq \sum_{e \in q^{\prime}} \boldsymbol{c}^{\boldsymbol{l}}(e)$,

so $q^{\prime}$ is either dominated w.r.t. $\boldsymbol{c}^{\boldsymbol{l}}$ or has an equivalent solution in $O P T^{\tilde{l}}$. Therefore, $O P T^{\tilde{l}}$ is a complete set of efficient solutions for $\mathcal{M P}(\boldsymbol{l})$

A fast way to use this result is to replace Step 3 of Algorithm 3 with Algorithm 4.

We loop through all $\boldsymbol{l} \in L$. In Lines 8 to 10, OPT $^{l}$ is found for the current $\boldsymbol{l}$ : either $(\mathcal{M P}(\boldsymbol{l}))$ is solved, or OPT $\boldsymbol{l}$ is set to the solution set of an already solved subproblem. For this purpose, we store one vector $\tilde{\boldsymbol{l}}^{\boldsymbol{h}}$ for each $h=1, \ldots, k$, which is updated in Line 13 whenever the value $l_{h}$ has changed, i.e. whenever $l_{i}$ was increased for some $i \leqq h$ in the respective for-loop.

When $l_{h}$ is increased in the for-loop, during the next execution of Line 8 , we have:

$$
\tilde{l}_{i}^{h}= \begin{cases}l_{i} & \begin{array}{l}
\text { for } i<h, \text { because } \tilde{\boldsymbol{l}}^{\mathbf{h}} \text { was updated } \\
\text { after the previous change of } l_{i},
\end{array} \\
l_{i}-1 & \begin{array}{l}
\text { for } i=h, \text { because } l_{h} \text { was increased, } \\
\text { but } \tilde{\boldsymbol{l}}^{\mathbf{h}} \text { is not updated yet, }
\end{array} \\
1=l_{i} & \begin{array}{l}
\text { for } i>h, \text { as, due to the nested for-loops, } l_{i} \\
\text { is set to } 1 \text { whenever } l_{h} \text { changes. }
\end{array}\end{cases}
$$

Hence, if no set in $O P T^{\tilde{I}^{h}}$ contains any element in $E_{l}^{h}$ the conditions of Lemma 13 are satisfied for $\tilde{\boldsymbol{l}}:=\tilde{\boldsymbol{l}}^{\boldsymbol{h}}$.

Corollary 14. Algorithm 3 with Algorithm 4 replacing Step 3 and the construction of L (Step 2) adjusted according to Lemma 12, finds a complete set of robust efficient solutions for (MOUCO). During its execution at most $\prod_{i=1}^{k}\left(\left\lceil\frac{|E|-\Gamma_{i}}{2}\right\rceil+1\right)$ deterministic subproblems have to be solved.

For problems with the following property, the number of subproblems to be solved can be reduced significantly.

Definition 15. An instance $(E, Q, \hat{C}, \Delta, \Gamma)$ has partial objectiveindependent element order if there exists a subset $J:=\left\{i_{1}, \ldots, i_{r}\right\} \subseteq$ $\{1, \ldots, k\}$ with $\overline{\text { Algorithm } 4 \text { Improved Step } 3 \text { of Algorithm 3: solve subproblems }}$ (with solution checking).

Input: an instance $I=(E, Q, \hat{C}, \Delta, \Gamma), \bar{\delta}_{i}^{j}$ and $E_{j}^{i} \forall i, j \in\{1, \ldots, k\}$, an index set $L$ of subproblems

Output: solution sets $\left(O P T^{\boldsymbol{l}}, \boldsymbol{l} \in L\right)$

1: $\tilde{\boldsymbol{l}}^{\mathbf{1}}:=(0, \ldots, 0)$

2: $h:=1$

3: for all $l_{1} \in L_{1}$ in increasing order do

for all $l_{2} \in L_{2}$ in increasing order do

for all $l_{k} \in L_{k}$ in increasing order do

$\boldsymbol{l}:=\left(l_{1}, \ldots, l_{k}\right)$

if $\tilde{\boldsymbol{l}}^{\mathbf{h}}=(0, \ldots, 0)$ or any of the sets in $O P T^{\tilde{l}_{\mathbf{h}}}$ contains

any element in $E_{l_{h}}^{h}$ then $(\mathcal{M P}(\boldsymbol{l}))$

Find a complete set of efficient solutions $O P T^{l}$ for

$$
\begin{array}{lc} 
& (\mathcal{M P}(\boldsymbol{l})) \\
10: & \text { else } O P T^{\boldsymbol{l}}:=O P T^{\tilde{l}^{\mathbf{h}}} \\
11: & \text { end if } \\
12: & \text { for } i=h, \ldots, k \text { do } \\
13: & \tilde{\boldsymbol{l}}:=\boldsymbol{l} \\
14: & \text { end for } \\
15: & h:=k \\
16: & \text { end for } \\
17: & \ldots \\
18: & h:=2 \\
19: & \text { end for } \\
20: & h:=1 \\
21: & \text { end for }
\end{array}
$$

- $\Gamma_{i_{1}}=\Gamma_{i_{2}}=\cdots=\Gamma_{i_{r}}$ and

- there exists an order of the elements in $E$, such that

$$
\delta_{e_{1}, i} \geqq \cdots \geqq \delta_{e_{|E|}, i} \forall i \in J \text {. }
$$
order.

If $J=\{1, \ldots, k\}$, the instance has objective-independent element

Example 16. Consider an instance with $E=\left\{e_{1}, e_{2}, e_{3}\right\}$ and

$\boldsymbol{\delta}_{\boldsymbol{e}_{1}}=(1,1,1)^{T}, \boldsymbol{\delta}_{\boldsymbol{e}_{2}}=(3,2,1)^{T}, \boldsymbol{\delta}_{\boldsymbol{e}_{3}}=(2,2,1)^{T}$.

Then $\delta_{e_{1}, i} \leq \delta_{e_{3}, i} \leq \delta_{e_{2}, i} \forall i=1, \ldots, 3$, hence the instance has objective-independent element order. With

$\delta_{\boldsymbol{e}_{1}}=(1,2,3)^{T}, \boldsymbol{\delta}_{\boldsymbol{e}_{2}}=(3,2,1)^{T}, \boldsymbol{\delta}_{\boldsymbol{e}_{3}}=(2,2,2)^{T}$

the instance does not have objective-independent element order, because $\delta_{e_{1}, 1}<\delta_{e_{3}, 1}$ and $\delta_{e_{3}, 3}<\delta_{e_{1}, 3}$. However, it has partial objective-independent element order, because, e.g., $\delta_{e_{2}, i} \leqq \delta_{e_{3}, i} \leqq$ $\delta_{e_{1}, i}$ for $i=2,3$.

Lemma 17. Let an instance $(E, Q, \hat{C}, \Delta, \Gamma)$ with partial objectiveindependent element order be given and let $J$ be the set of indices defined in Definition 15. Then the nested for-loops changing $l_{i_{1}}, \ldots, l_{i_{r}}$ in Algorithm 4 can be replaced by a single for-loop. The number of solved deterministic subproblems in Algorithm 3 with Algorithm 4 (with replaced for-loops) as Step 3 and L adjusted according to Lemma 12 is then less than or equal to

$\left(\left\lceil\frac{|E|-\Gamma_{i_{1}}}{2}\right\rceil+1\right)$ if $J=\{1, \ldots, k\}$

$\left(\left\lceil\frac{|E|-\Gamma_{i_{1}}}{2}\right\rceil+1\right) \cdot \prod_{i \in\{1, \ldots, k\} \backslash\left\{i_{1}, \ldots, i_{r}\right\}}\left(\left\lceil\frac{|E|-\Gamma_{i}}{2}\right\rceil+1\right)$ otherwise.

Proof. In the proof of Theorem 10 we have shown that for each $q \in Q$ there exists an $\boldsymbol{l} \in L$ with $\boldsymbol{z}^{\mathbf{R}}(q)=\mathbf{g}^{\mathbf{l}}(q)$. We show that there always is such an $\boldsymbol{l}$ with $l_{i_{1}}=\cdots=l_{i_{r}}$.

https://doi.org/10.1016/j.ejor.2017.12.018 
Since there exists an order of the elements in $E$ such that $\delta_{e_{1}, i} \geqq \cdots \geqq \delta_{e_{|E|}, i} \forall i \in J$, we can choose the sets $E_{j}^{i}$ such that $E_{j}^{i_{1}}=$ $\cdots=E_{j}^{i_{r}} \forall j=1, \ldots,|E|$. In the proof of Theorem 10 we choose $\bar{l}_{i}$ such that $E_{\bar{l}_{i}}^{i} \cap q$ has exactly $\Gamma_{i}$ elements. With $\Gamma_{i_{1}}=\cdots=\Gamma_{i_{r}}$ it follows $\bar{l}_{i_{1}}=\cdots=\bar{l}_{i_{r}}$. Hence, we have $\boldsymbol{z}^{\mathbf{R}}(q)=\mathbf{g}^{\bar{l}}(q)$ and $\bar{l}_{i_{1}}=\cdots=\bar{l}_{i_{r}}$.

It follows that the nested for-loops changing $l_{i_{1}}, \ldots, l_{i_{r}}$ can be replaced by a single for-loop, which leads directly to the stated number of subproblems. $\square$

\subsection{Bottleneck approach}

In the algorithms presented in the previous section, the number of subproblems that have to be solved increases with decreasing values of $\Gamma_{i}$. In this section we present a method whose complexity decreases with decreasing values of $\Gamma_{i}$. Its idea is to transfer (MOUCO) with $k$ objectives into a deterministic combinatorial optimization problem of the same kind with $\sum_{i=1}^{k}\left(\Gamma_{i}+1\right)$ objective functions, some of which are bottleneck functions instead of sum functions. The concept is particularly useful if an efficient algorithm for solving the deterministic multi-objective problem with sum and bottleneck functions is available. As an example we present such an algorithm for the shortest path problem in Section 3.2.2.

3.2.1. Bottleneck approach for cardinality-constrained uncertain combinatorial optimization problems

We first explain the approach for the single-objective uncertain problem $\left(\min _{q \in Q} z(q), c \in \mathcal{U}^{c c}\right)$ with $\mathcal{U}^{c c}$ as given in Eq. (1). The robust counterpart (MORCO) then reduces to

$$
\text { (RCO) } \min _{q \in Q}\left(z^{\mathrm{R}}(q)=\max _{c \in \mathcal{U}^{c c}} \sum_{e \in q} c(e)\right) \text {. }
$$

Definition 18. For a subset $q \subseteq E$ and given interval lengths $\delta_{e}$ for all $e \in E$, we sort the elements in $q$ by decreasing interval lengths and define $j$-max $\operatorname{maq}_{e \in q} \delta_{e}$ as the interval length of the $j$ th element according to this sorting.

Theorem 19. Every optimal solution for (RCO) is an efficient solution for the deterministic multi-objective problem

$(D C O) \min _{q \in Q}\left(z^{\mathbf{D}}(q):=\left(\begin{array}{c}\sum_{e \in q} \hat{c}_{e} \\ \max _{e \in q} \delta_{e} \\ 2-\max _{e \in q} \delta_{e} \\ \vdots \\ \Gamma-\max _{e \in q} \delta_{e}\end{array}\right)\right)$

Proof. Recall that any feasible set $q \in Q$ has maximal cost if the cost of its $\Gamma$ elements with the largest cost intervals take their maximal values. Let $q$ be an optimal solution for (RCO). Assume that $q$ is not efficient for (DCO). Then there exists a solution $q^{\prime} \in Q$ that dominates $q$ and it follows

$\sum_{e \in q^{\prime}} \hat{c}_{e} \leqq \sum_{e \in q} \hat{c}_{e}$ and

$j-\max \delta_{e} \leqq j-\max \delta_{e} \forall j=1, \ldots, \Gamma$, with at least one inequality $\Rightarrow z^{\mathrm{R}}\left(q^{\prime}\right)=\sum_{e \in q^{\prime}} \hat{c}_{e}+\sum_{j=1}^{\Gamma} j-\max _{e \in q^{\prime}} \delta_{e}<\sum_{e \in q} \hat{c}_{e}+\sum_{j=1}^{\Gamma} j-\max _{e \in q} \delta_{e}=z^{\mathrm{R}}(q)$.

This contradicts $q$ being optimal for (RCO).

The reverse of Theorem 19 does not hold: there exist efficient solutions for (DCO), which are not optimal for (RCO), as the following example shows.
Example 20. Let $G$ be a graph that consists of two disjoint paths $q, q^{\prime}$ from $s$ to $t$ with three edges each. Let the cost interval of all edges in $q$ be $[1,1]$ and of all edges in $q^{\prime}$ be $[0,1]$ and let $\Gamma=2$. Then both paths are efficient solutions for (DCO), because

$$
\boldsymbol{z}^{\mathbf{D}}(q)=(3,0,0) \not \leq(0,1,1)=\boldsymbol{z}^{\mathbf{D}}\left(q^{\prime}\right)
$$

and $\boldsymbol{z}^{\mathbf{D}}\left(q^{\prime}\right)=(0,1,1) \not 3(3,0,0)=\boldsymbol{z}^{\mathbf{D}}(q)$.

But only $q^{\prime}$ is robust efficient, because

$z^{\mathrm{R}}\left(q^{\prime}\right)=2<3=z^{\mathrm{R}}(q)$.

Lemma 21. A complete set of efficient solutions for (DCO) contains at least one optimal solution for (RCO).

Proof. Let $Q^{\prime} \subseteq Q$ be a complete set of efficient solutions for (DCO). Assume, that (RCO) has an optimal solution $q$ that is not contained in $Q^{\prime}$. According to Theorem 19, $q$ is an efficient solution for (DCO), so $Q^{\prime}$ contains a solution $q^{\prime}$ with

$$
\begin{aligned}
\left(\begin{array}{c}
\sum_{e \in q} \hat{c}_{e} \\
\max _{e \in q} \delta_{e} \\
2-\max _{e \in q} \delta_{e} \\
\vdots \\
\Gamma-\max _{e \in q} \delta_{e}
\end{array}\right) & =\left(\begin{array}{c}
\sum_{e \in q^{\prime}} \hat{c}_{e} \\
\max _{e \in q^{\prime}} \delta_{e} \\
2-\max _{e \in q^{\prime}} \delta_{e} \\
\vdots \\
\Gamma-\max _{e \in q^{\prime}} \delta_{e}
\end{array}\right) \\
& \Rightarrow z^{\mathrm{R}}(q)=\sum_{e \in q} \hat{c}_{e}+\sum_{j=1}^{\Gamma} j-\max _{e \in q} \delta_{e}=z^{\mathrm{R}}\left(q^{\prime}\right)
\end{aligned}
$$

and $q^{\prime}$ is optimal for (RCO).

Now, we transfer this approach to the multi-objective case. For a problem with $k$ objectives, we construct a deterministic problem with $m:=\sum_{i=1}^{k}\left(\Gamma_{i}+1\right)$ objectives.

Theorem 22. Every efficient solution for the multi-objective robust counterpart (MORCO) is an efficient solution for the deterministic multi-objective problem

$\left(\right.$ MODCO) $\min _{q \in Q}\left(z^{\mathbf{D}}(q):=\left(\begin{array}{c}\sum_{e \in q} \hat{c}_{e, 1} \\ \max _{e \in q} \delta_{e, 1} \\ 2-\max _{e \in q} \delta_{e, 1} \\ \vdots \\ \Gamma_{1}-\max _{e \in q} \delta_{e, 1} \\ \sum_{e \in q} \hat{c}_{e, 2} \\ \max _{e \in q} \delta_{e, 2} \\ \vdots \\ \Gamma_{k}-\max _{e \in q} \delta_{e, k}\end{array}\right)\right)$.

A complete set of solutions for (MODCO) contains a complete set of solutions for (MORCO).

Proof. Let $q$ be an efficient solution for (MORCO). Assume that $q$ is not efficient for (MODCO). Analogously to the proof of Theorem 19, there is a solution $q^{\prime} \in Q$ dominating $q$ and it follows that $z_{i}^{\mathrm{R}}\left(q^{\prime}\right)<$ $z_{i}^{\mathrm{R}}(q)$ for at least one $i \in\{1, \ldots, k\}$, which contradicts $q$ being efficient for (MORCO).

Assume now, that $q \notin Q^{\prime}$ with $Q^{\prime}$ being a complete set of efficient solutions for (MODCO). Since $q$ is efficient for (MODCO), there is a solution $q^{\prime} \in Q^{\prime}$ equivalent to $q$ w.r.t. the objective function of (MODCO) and it follows $\boldsymbol{z}^{\mathbf{R}}(q)=\boldsymbol{z}^{\mathbf{R}}\left(q^{\prime}\right)$ analogously to the proof of Lemma 21.

With an algorithm to solve (MODCO) and a method to filter the obtained solutions we can now find a complete set of robust efficient solutions for the uncertain problem. In the case of a single-objective uncertain problem, Gorski, Klamroth, and Ruzika (2012) introduced an algorithm to solve (DCO).

https://doi.org/10.1016/j.ejor.2017.12.018 
3.2.2. Label setting algorithm (LSA) for (MOUSP)

In this section, we show how to apply the bottleneck approach to the cardinality-constrained uncertain shortest path problem. We propose an adjustment of standard multi-objective labeling algorithms (label setting or label correcting) to find a complete set of robust efficient solutions

Let (MOUSP) be defined as in Section 2.1, i.e., $E$ is the edge set of a graph and $Q$ the set of simple paths from a given start node $s$ to a given end node $t$. Additionally we assume non-negative edge costs $\left(\boldsymbol{c}(e) \geqq 0 \forall e \in E, \boldsymbol{c} \in \mathcal{U}^{m c c}\right)$ and adjust a label setting algorithm as an example.

We first recall the definition of a label, which is used in common multi-objective labeling algorithms. A label $l=\left(\boldsymbol{y}, v^{\prime}, l^{\prime}\right)$ at a node $v$ consists of

- a cost vector $\boldsymbol{y}$, here $\boldsymbol{y}=\left(y_{1}, \ldots, y_{m}\right)^{T}$,

- a predecessor node $v^{\prime}$, and

- a predecessor label $l^{\prime}$.

Every label at a node $v \neq s$ with predecessor node $v^{\prime}$ represents a path $q$ from $s$ to $v$ whose last edge is $\left(v^{\prime}, v\right)$. That means that its cost equals the cost of $q$ and its predecessor label $l^{\prime}$ represents the subpath of $q$ from $s$ to $v^{\prime}$. We assume here, that no parallel edges exist, such that $v$ and $v^{\prime}$ uniquely define an edge $\left(v^{\prime}, v\right)$. If paralle edges have to be considered, the respective edge can be contained in the label as well. The labels are constructed iteratively from existing labels at the predecessor nodes and can at any time be either temporary or permanent.

Algorithm 5 is a label setting algorithm for solving (MODCO)

Algorithm 5 Label setting algorithm to solve (MODCO) for the shortest path problem.

Input: an instance $I=(E, Q, \hat{C}, \Delta, \Gamma)$ of (MOUSP)

Output: permanent labels at $t$, representing a complete set of efficient solutions for instance $I$ of (MODCO)

1: Set $m:=\sum_{i=1, k}\left(\Gamma_{i}+1\right)$.

2: Create a temporary label $l_{0}$ with cost $(0, \ldots, 0)^{T}$ at node $s$.

3: while there exists at least one temporary label do

4: $\quad$ Select a temporary label $l^{\prime}$ (at any node $v^{\prime}$ ) with minimal aggregate cost $\sum_{j=1, \ldots, m} y_{j}^{\prime}$ and make it permanent.

for all outgoing edges $\left(v^{\prime}, v\right)$ of $v^{\prime}$ do

Create a new temporary label $l$ at $v$ by Algorithm 6 .

if the cost of $l$ is dominated by or equal to the cost of another label at $v$ then

$$
\text { 8: } \quad \text { Delete } l \text {. }
$$

9: $\quad$ else if $l$ dominates any temporary labels at $v$ then

10: Delete these labels.

11: end if

12: end for

13: end while

for the shortest path problem. It is based on the label setting algorithm by Martins (1984) for multi-objective shortest path problems, but we make the following adjustments:

1. In Step 4 a label must be chosen whose cost is not dominated by the cost of any other temporary label. In the algorithm by Martins (1984) the lexicographically smallest label is chosen. Based on Iori, Martello, and Pretolani (2010), we choose the label with the smallest aggregate cost function $\sum_{j=1, \ldots, m} y_{j}$ instead.

2. In multi-objective label setting algorithms with only sum functions (as considered by Martins, 1984) a new label $l=\left(\boldsymbol{y}, v^{\prime}, l^{\prime}\right)$ at $v$ is created by adding the cost $\boldsymbol{y}^{\prime}$ of the predecessor label $l^{\prime}$ to the edge cost. For min-max functions the (entry-wise) maximum of the edge cost and the predecessor label's cost is taken (see Gandibleux, Beugnies, \& Randriamasy, 2006). To solve (MODCO) we need a new way to construct the labels: let $n_{i}:=1+\sum_{j=1, \ldots,(i-1)}\left(\Gamma_{j}+1\right)$ denote the index of the first objective of (MODCO) associated with the original objective $z_{i}$ of (MORCO). For the sum objective functions, we add the nominal cost $\hat{c}_{e, i}$ of the edge $e:=\left(v^{\prime}, v\right)$ to the corresponding predecessor cost entry $y_{n_{i}}^{\prime}$. For the $j$-max objective functions, we compare for each objective $z_{i}$ the interval length $\delta_{e, i}$ of $e$ to each of the $\Gamma_{i}$ longest interval lengths so far $y_{n_{i}+1}^{\prime}, \ldots, y_{n_{i}+\Gamma_{i}}^{\prime}$ and insert it at the right position (see Algorithm 6). We will use the

Algorithm 6 Step 6 of Algorithm 5: create a new temporary label. Input: an instance $I=(E, Q, \hat{C}, \Delta, \Gamma)$, an edge $\left(v^{\prime}, v\right) \in E$, a label $l^{\prime}$ with cost $\boldsymbol{y}^{\prime}$ at $v^{\prime}$

Output: a new label $l$ at $v$ with predecessor label $l^{\prime}$

1: for $i=1, \ldots, k$ do

2: Set $n_{i}:=1+\sum_{j=1, \ldots .(i-1)}\left(\Gamma_{i}+1\right)$.

$y_{n_{i}}:=y_{n_{i}}^{\prime}+\hat{c}_{\left(v^{\prime}, v\right), i}$

$a:=1$

while $a \leqq \Gamma_{i}$ do

if $\delta_{\left(v^{\prime}, v\right), i}>y_{n_{i}+a}^{\prime}$ then

$y_{n_{i}+a}:=\delta_{\left(v^{\prime}, v\right)}$

for $b:=a+1, \ldots, \Gamma_{i}$ do $y_{n_{i}+b}:=y_{n_{i}+b-1}^{\prime}$

end for

$a:=\Gamma_{i}+1$

else

$y_{n_{i}+a}:=y_{n_{i}+a}^{\prime}$

$a:=a+1$

end if

end while

: end for

7: Create the temporary label $l:=\left(\left(y_{0}, \ldots, y_{m}\right)^{T}, v^{\prime}, l^{\prime}\right)$ at node $v$.

following notation: $\boldsymbol{y}:=\boldsymbol{y}^{\prime} \oplus\left(\hat{\boldsymbol{c}}_{\boldsymbol{e}}, \boldsymbol{\delta}_{\boldsymbol{e}}\right)$.

3. In the algorithm by Martins (1984) a newly created label is only deleted if it is dominated by a label at the same node. We delete the new label even if another label with equal cost exists at the same node, because we are only looking for a complete set of efficient solutions. This is also the reason why we do not need to consider hidden labels, which Gandibleux et al. (2006) introduced for problems with bottleneck functions. Since new labels with the same cost as existing labels are immediately deleted, Algorithm 5 works even without the assumption that no cycles of cost $(0, \ldots, 0)$ exist.

Example 23. We show the first steps of Algorithm 5 with the instance given in Example 7 as input.

1. In Lines 1 and $2, m$ is set to $(2+1)+(2+1)=6$ and a temporary label $l_{0}$ with cost $(0,0,0,0,0,0)^{T}$ is created at node $v_{1}$.

2. The label $l_{0}$ is made permanent in Line 4 and new temporary labels are created at the nodes $v_{2}, v_{3}$ :

$l_{2}^{1}$ at $v_{2}$ with cost $(2,1,0,1,1,0)^{T}$ representing $\left\{\left(v_{1}, v_{2}\right)\right\}$ $l_{3}^{1}$ at $v_{3}$ with cost $(4,3,0,3,5,0)^{T}$ representing $\left\{\left(v_{1}, v_{3}\right)\right\}$.

We now have one permanent label $l_{0}$ and two temporary labels $l_{2}^{1}, l_{3}^{1}$. The aggregated cost of $l_{2}^{1}$ is smaller than the aggregated cost of $l_{3}^{1}$.

3. Because of its smaller aggregated cost, $l_{2}^{1}$ is made permanent in the next iteration of Line 4 . New labels are created:

$l_{3}^{2}$ at $v_{3}$ with cost $(3,1,1,2,5,1)^{T}$ representing

$\left\{\left(v_{1}, v_{2}\right),\left(v_{2}, v_{3}\right)\right\}$

$l_{4}^{1}$ at $v_{4}$ with cost $(5,4,1,5,1,1)^{T}$ representing

https://doi.org/10.1016/j.ejor.2017.12.018 
$\left\{\left(v_{1}, v_{2}\right),\left(v_{2}, v_{4}\right)\right\}$

$l_{5}^{1}$ at $v_{2}$ with cost $(5,5,1,3,5,1)^{T}$ representing

$\left\{\left(v_{1}, v_{2}\right),\left(v_{2}, v_{5}\right)\right\}$.

As an example, we look at the creation of $l_{3}^{2}$ in detail: the cost vector of $l_{2}^{1}$ is $(2,1,0,1,1,0)^{T}=\boldsymbol{y}^{\prime}$. We obtain

$$
\begin{aligned}
\boldsymbol{y}^{\prime} \oplus\left(\hat{\boldsymbol{c}}_{\left(v_{2}, v_{3}\right)}, \boldsymbol{\delta}_{\left(v_{2}, v_{3}\right)}\right)= & \left(\begin{array}{l}
2 \\
1 \\
0 \\
1 \\
1 \\
0
\end{array}\right) \oplus\left(\left(\begin{array}{l}
1 \\
1
\end{array}\right),\left(\begin{array}{l}
1 \\
5
\end{array}\right)\right) \\
= & \left(\begin{array}{c}
y_{1}^{\prime}+\hat{c}_{\left(v_{2}, v_{3}\right), 1} \\
y_{2}^{\prime} \\
\delta_{\left(v_{2}, v_{3}\right), 1}^{\prime} \\
y_{4}^{\prime}+\hat{c}_{\left(v_{2}, v_{3}\right), 2} \\
\delta_{\left(v_{2}, v_{3}, 2\right.}^{\prime} \\
y_{5}^{\prime}
\end{array}\right)=\left(\begin{array}{l}
3 \\
1 \\
1 \\
2 \\
5 \\
1
\end{array}\right),
\end{aligned}
$$

because $y_{2}^{\prime} \geqq \delta_{\left(v_{2}, v_{3}\right), 1}>y_{3}^{\prime}$ and $\delta_{\left(v_{2}, v_{3}\right), 2}>y_{5}^{\prime}$. The cost vectors of the two labels $l_{3}^{1}, l_{3}^{2}$ at $v_{3}$ are compared to each other. As none dominates the other, both are kept. The labels $l_{0}, l_{2}^{1}$ are now permanent. We have four temporary labels $l_{3}^{1}, l_{3}^{2}, l_{4}^{1}, l_{5}^{1}$, among which $l_{3}^{2}$ has the smallest aggregated cost.

After several iterations of Lines $4-13$, there do not exist any temporary labels. Algorithm 5 returns 3 permanent labels at node $v_{6}$ :

one with cost $(8,4,1,7,1,1)^{T}$ representing

$q_{1}=\left\{\left(v_{1}, v_{2}\right),\left(v_{2}, v_{4}\right),\left(v_{4}, v_{6}\right)\right\}$

one with cost $(6,3,2,6,5,5)^{T}$ representing

$q_{2}=\left\{\left(v_{1}, v_{2}\right),\left(v_{2}, v_{3}\right),\left(v_{3}, v_{5}\right),\left(v_{5}, v_{6}\right)\right\}$,

one with cost $(8,3,1,6,5,5)^{T}$ representing

$q_{3}=\left\{\left(v_{1}, v_{2}\right),\left(v_{2}, v_{3}\right),\left(v_{3}, v_{4}\right),\left(v_{4}, v_{6}\right)\right\}$.

In Algorithm 7 non-dominated paths according to their worst case

$\overline{\text { Algorithm } 7 \text { LSA for the shortest path problem with cardinality- }}$ constrained uncertainty.

Input: an instance $I=(E, Q, \hat{C}, \Delta, \Gamma)$ of (MOUSP)

Output: a complete set of robust efficient solutions for

1: Solve (MODCO) with Algorithm 5.

2: For every permanent label $l$ in $t$ compute the worst case costs $\boldsymbol{z}^{\mathbf{R}}(q)$ of its represented path $q$ by $z_{i}^{\mathrm{R}}(q):=\sum_{i=n_{i}, \ldots, n_{i}+\Gamma_{i}} y_{i}$ and choose the non-dominated ones.

3: Obtain the represented paths by backtracking the predecessor labels.

cost will be identified from the obtained labels, see Example 29.

Lemma 24. In Algorithm 5 for every label $l=\left(\boldsymbol{y}, v^{\prime}, l^{\prime}\right)$ at a node $v$ there exists a path $q$ from $s$ to $v$ with $\boldsymbol{y}=\boldsymbol{z}^{\mathbf{D}}(q)$.

Proof. We show the statement by induction:

The first label has cost $(0, \ldots, 0)$ and represents the path only consisting of node $s$.

Let $\boldsymbol{y}^{\prime}=\left(y_{1}^{\prime}, \ldots, y_{m}^{\prime}\right)$ be the cost of the predecessor label $l^{\prime}$ and assume that $\boldsymbol{y}^{\prime}$ equals the cost $\boldsymbol{z}^{\mathbf{D}}\left(q^{\prime}\right)$ of a path $q^{\prime}$ from $s$ to $v^{\prime}$. Let $q:=q^{\prime} \cup\left(v^{\prime}, v\right)$. Then we have

$\forall i=1, \ldots, k: y_{n_{i}}=y_{n_{i}}^{\prime}+\hat{c}_{\left(v^{\prime}, v\right), i}=\sum_{e \in q^{\prime}} \hat{c}_{e, i}+\hat{c}_{\left(v^{\prime}, v\right), i}=\sum_{e \in q} \hat{c}_{e, i}$.

Further, we distinguish two cases for all $i=1, \ldots, k$ :
- Case 1: $\delta_{\left(v^{\prime}, v\right), i} \leqq y_{n_{i}+a}^{\prime} \forall a=1, \ldots, \Gamma_{i}$. In this case the $\Gamma_{i}$ edges $e$ with biggest intervals $\delta_{e, i}$ of $q^{\prime}$ and $q^{\prime} \cup\left(v^{\prime}, v\right)$ are the same and $y_{n_{i}+a}=y_{n_{i}+a}^{\prime}$ for all $a=1, \ldots, \Gamma_{i}$. Therefore, $\left(y_{n_{i}}, \ldots, y_{n_{i}+\Gamma_{i}}\right)=$ $\left(z_{n_{i}}^{\mathrm{D}}(q), \ldots, z_{n_{i}+\Gamma_{i}}^{\mathrm{D}}(q)\right)$.

- Case 2: Either $\delta_{\left(v^{\prime}, v\right), i}>y_{n_{i}+a}^{\prime}$ for $a=1$ or $\exists a \in\left\{2, \ldots, \Gamma_{i}\right\}$ with $y_{n_{i}+a-1}^{\prime} \geqq \delta_{\left(v^{\prime}, v\right), i}>y_{n_{i}+a}^{\prime}$. Then

$\forall b<a: y_{n_{i}+b}=y_{n_{i}+b}^{\prime}$ and $b-\max _{e \in q^{\prime}} \delta_{e, i}=b-\max _{e \in q^{\prime} \cup\left(v^{\prime}, v\right)} \delta_{e, i}$

for $b=a: y_{n_{i}+b}=\delta_{\left(v^{\prime}, v\right), i}=b-\max _{e \in q^{\prime} \cup\left(v^{\prime}, v\right)} \delta_{e, i}$

$\forall b$ : with $\Gamma_{i} \geqq b>a: y_{n_{i}+b}=y_{n_{i}+b-1}^{\prime}=b-\max _{e \in q \cup\left(v^{\prime}, v\right)} \delta_{e, i}$

It follows $\left(y_{1}, \ldots, y_{m}\right)=\boldsymbol{z}^{\mathbf{D}}(q)$.

In the deterministic case with only sum functions, subpaths of efficient paths are efficient as well, which plays an important role in the proof of Martin's algorithm. If some of the objective functions are bottleneck functions, this property does not hold any more (Gandibleux et al., 2006). In our case, since we only look for a complete set of efficient solutions, the weaker property given in Lemma 26 is sufficient (this was observed but not proven by Iori et al. (2010)).

We use the following notation to specify subpaths.

Notation 25. Let $q$ be a simple path and $v, w$ two nodes on $q$ ( $v$ before $w$ ). Let then $q_{v, w}$ denote the part of $q$ from node $v$ to node $w$.

Lemma 26. Let $q$ from $s$ to $t$ be an efficient path with respect to $z^{\mathbf{D}}$ and $v, w$ two nodes on $q$ ( $v$ before $w$ ). Then either $q_{v, w}$ is an efficient path from $v$ to $w$ or there exists an efficient path $p$ from $v$ to $w$ such that $q^{\prime}:=q_{s, v} \cup p \cup q_{w, t}$ is equivalent to $q$.

Proof. Assume that $q_{v, w}$ is not efficient w.r.t $\boldsymbol{z}^{\mathbf{D}}$. Then there exists an efficient path $p$ from $v$ to $w$ that dominates $q_{v, w}$. We have

$$
\begin{aligned}
\sum_{e \in q^{\prime}} \hat{\boldsymbol{c}}_{\boldsymbol{e}} & =\sum_{\boldsymbol{e} \in q_{s, v}} \hat{\boldsymbol{c}}_{\boldsymbol{e}}+\sum_{\boldsymbol{e} \in p} \hat{\boldsymbol{c}}_{\boldsymbol{e}}+\sum_{\boldsymbol{e} \in q_{w, t}} \hat{\boldsymbol{c}}_{\boldsymbol{e}} \leqq \sum_{q_{s, v}} \hat{\boldsymbol{c}}_{\boldsymbol{e}}+\sum_{\boldsymbol{e} \in q_{v, w}} \hat{\boldsymbol{c}}_{\boldsymbol{e}}+\sum_{e \in q_{w, t}} \hat{\boldsymbol{c}}_{\boldsymbol{e}} \\
& =\sum_{\boldsymbol{e} \in q} \hat{\boldsymbol{c}}_{\boldsymbol{e}} .
\end{aligned}
$$

As $p$ dominates $q_{v, w}$, it follows $\forall i=1, \ldots, k, a=1, \ldots, \Gamma_{i}$ : $a-\max _{e \in p} \delta_{e, i} \leqq a-\max _{e \in q_{\nu, w}} \delta_{e, i}, \quad$ and hence $a-\max _{e \in q^{\prime}} \delta_{e, i} \leqq$ $a-\max _{e \in q} \delta_{e, i} \forall i=1, \ldots, k, a=1, \ldots, \Gamma_{i}$.

It follows $\boldsymbol{z}^{\mathbf{D}}\left(q^{\prime}\right) \leqq \boldsymbol{z}^{\mathbf{D}}(q)$ and we conclude $\boldsymbol{z}^{\mathbf{D}}\left(q^{\prime}\right)=\boldsymbol{z}^{\mathbf{D}}(q)$, because $q$ is efficient with respect to $z^{\mathbf{D}}$. $\square$

Theorem 27. When Algorithm 5 (with Algorithm 6 as Step 6) stops, the permanent labels at $t$ represent a complete set of efficient solutions for (MODCO).

Proof. We have to show that each permanent label at $t$ represents an efficient path from $s$ to $t$ and that for each efficient path $q$ from $s$ to $t$ a permanent label at $t$ representing $q$ or an equivalent path exists.

The proof of the first part is analogous to the proof by Ehrgott (2006) of the multi-objective label setting algorithm by Martins (1984). For substituting the lexicographic order with the aggregate cost order we refer to Iori et al. (2010).

Now, we show that for each efficient path $q$ from $s$ to $t$ a permanent label at $t$ representing $q$ or an equivalent path exists. Assume that we have an efficient path $q$ from $s$ to $t$, such that there is no permanent label $l$ at $t$ with label costs $\boldsymbol{y}=\boldsymbol{z}^{\mathbf{D}}(q)$. Consider the predecessor node $v^{\prime}$ of $t$ on $q$. From Lemma 26 it follows that there is an efficient path $p$ from $s$ to $v^{\prime}$ with $\boldsymbol{z}^{\mathbf{D}}\left(p \cup\left(v^{\prime}, t\right)\right)=\boldsymbol{z}^{\mathbf{D}}(q)$.

If there exists a permanent label $l^{\prime}$ at $v^{\prime}$ with label costs $\boldsymbol{y}^{\prime}=\boldsymbol{z}^{\mathbf{D}}(p)$, then, after it was made permanent in Line 4 , a new

https://doi.org/10.1016/j.ejor.2017.12.018 


\section{A. Publications}

label $\bar{l}$ at node $t$ with label costs $\overline{\boldsymbol{y}}=\boldsymbol{y}^{\prime} \oplus\left(\hat{\boldsymbol{c}}_{\left(v^{\prime}, \boldsymbol{t}\right)}, \boldsymbol{\delta}_{\left(v^{\prime}, \boldsymbol{t}\right)}\right)$ was constructed in Line 6 . It follows

$\overline{\boldsymbol{y}}=\boldsymbol{y}^{\prime} \oplus\left(\hat{\boldsymbol{c}}_{\left(v^{\prime}, \boldsymbol{t}\right)}, \boldsymbol{\delta}_{\left(v^{\prime}, \boldsymbol{t}\right)}\right)=\boldsymbol{z}^{\mathbf{D}}(p) \oplus\left(\hat{\boldsymbol{c}}_{\left(v^{\prime}, \boldsymbol{t}\right)}, \boldsymbol{\delta}_{\left(v^{\prime}, \boldsymbol{t}\right)}\right)=\boldsymbol{z}^{\mathbf{D}}\left(p \cup\left(v^{\prime}, t\right)\right)$ $=\boldsymbol{z}^{\mathbf{D}}(q)$.

Consider the first label with $\operatorname{cost} \boldsymbol{z}^{\mathbf{D}}(q)$ that was constructed at node $t$. If this label was deleted again, its cost vector is dominated, which contradicts the efficiency of $q$. If it was not deleted, then it was made permanent, which contradicts our assumption that no permanent label with costs $\boldsymbol{z}^{\mathbf{D}}(q)$ exists at $t$.

Therefore, there is no permanent label at the predecessor node $v^{\prime}$ of $t$ with costs $\boldsymbol{y}^{\prime}$ such that $\boldsymbol{y}^{\prime} \oplus\left(\hat{\boldsymbol{c}}_{\boldsymbol{e}}, \boldsymbol{\delta}_{\boldsymbol{e}}\right)=\boldsymbol{z}^{\mathbf{D}}(q)$. In the same way, we can show that there is no permanent label at the predecessor node $v^{\prime \prime}$ of $v^{\prime}$ with costs $\boldsymbol{y}^{\prime \prime}$ such that

$$
\begin{aligned}
& \left(\boldsymbol{y}^{\prime \prime} \oplus\left(\hat{\boldsymbol{c}}_{\left(v^{\prime}, v^{\prime}\right)}, \boldsymbol{\delta}_{\left(v^{\prime \prime}, v^{\prime}\right)}\right)\right) \oplus\left(\hat{\boldsymbol{c}}_{\left(v^{\prime}, \boldsymbol{t}\right)}, \boldsymbol{\delta}_{\left(v^{\prime}, \boldsymbol{t}\right)}\right)=\boldsymbol{y}^{\prime} \oplus\left(\hat{\boldsymbol{c}}_{\left(v^{\prime}, \boldsymbol{t}\right)}, \boldsymbol{\delta}_{\left(v^{\prime}, \boldsymbol{t}\right)}\right) \\
& \quad=\boldsymbol{z}^{\mathbf{D}}(q) .
\end{aligned}
$$

By induction it follows that there is no permanent label at node $s$ with cost $(0, \ldots, 0)$, which is a contradiction, because such a label is constructed in Line 2 of the algorithm and made permanent during the first execution of Line 4.

We conclude that for each efficient path $q$ from $s$ to $t$ there exists a permanent label at $t$ representing $q$ or a path that is equivalent to $q$. Furthermore, each permanent label at $t$ represents an efficient path from $s$ to $t$. Therefore, the paths represented by the permanent labels are a complete set of efficient solutions. $\square$

To find a a complete set of robust efficient solutions we have to filter the solutions obtained by the labeling algorithm (see Algorithm 7).

Corollary 28. Algorithm 7 finds a complete set of robust efficient solutions for an instance $I=(E, Q, \hat{C}, \Delta, \boldsymbol{\Gamma})$ of (MOUSP) with $\hat{C}$ being entry-wise non-negative.

Example 29. Consider the instance given in Example 7. From the permanent labels returned by Algorithm 5 (see Example 23), the worst costs of their represented paths are computed:

$z^{\mathbf{R}}\left(q_{1}\right)=(8+4+1,7+1+1)^{T}=(13,9)^{T}$

$z^{\mathbf{R}}\left(q_{2}\right)=(6+3+2,6+5+5)^{T}=(11,16)^{T}$

$\boldsymbol{z}^{\mathbf{R}}\left(q_{3}\right)=(8+3+1,6+5+5)^{T}=(12,16)^{T}$.

Since $\boldsymbol{z}^{\mathbf{R}}\left(q_{3}\right)$ is dominated by $\boldsymbol{z}^{\mathbf{R}}\left(q_{2}\right)$, only the paths $q_{1}$ and $q_{2}$ are returned by Algorithm 7 .

\section{Experimental evaluation}

In this paper, we presented two approaches to find a complete set of robust efficient solutions for (MOUCO). DSA solves the uncertain problem, assuming that we know how to solve the deterministic multi-objective problem. To use the bottleneck approach we need a method to solve a deterministic multi-objective problem with several objective functions, some of which are sums and some of which are bottleneck functions. We introduced such an algorithm for the shortest path problem (LSA) and, hence, we test our approaches on the shortest path problem (MOUSP).

\subsection{Hazardous material transportation}

We test our algorithms for (MOUSP) on a hazardous material transportation instance: when transporting hazardous materials, on one hand, the shipping company wants to minimize travel time, distance or fuel costs. On the other hand, if an accident hap-

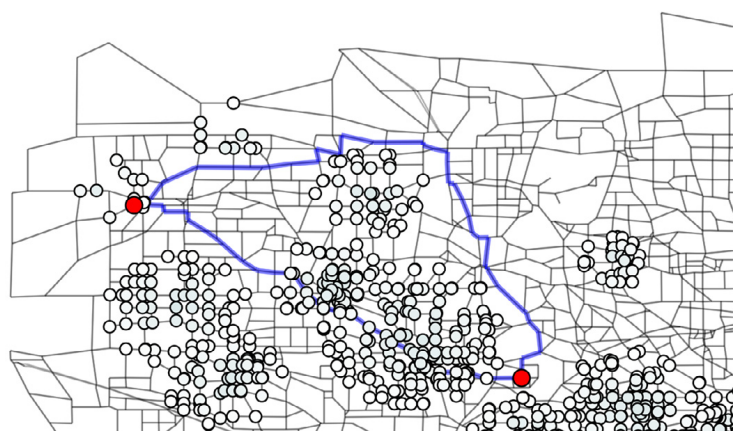

Fig. 3. Section of the Chicago regional road network with distribution of population (see Kuhn et al., 2016). The two big (red) dots show start and end node chosen for our experiments and two exemplary robust efficient paths are marked with thick (blue) lines.

pens, environment and population are exposed to the hazardous material. Hence, another objective is to keep the risk and negative impacts of accidents to a minimum. Erkut, Tjandra, and Verter (2007) give an overview about objectives for hazardous material transportation and about approaches for estimating the risk and the impacts of an accident.

For our experiments we consider the travel time and the population affected by a potential accident. We assume a nominal travel time on each road and a potential delay resulting from congestion or incidents like accidents or road construction works on some of the roads. We further assume a nominal population level, which can be increased locally by events like fairs or sport events, or due to regular shifts in population during the workday.

Our problem instance for hazardous material transportation is based on the instance used by Kuhn et al. (2016) to test an algorithm for bi-objective shortest path problems with only one uncertain objective. The underlying network (Chicago-regional) is a sector of the Chicago region road network available from Bar-Gera, Kwon, Li, and Stabler . The sector contains 1301 nodes and 4091 edges.

To obtain plausible travel times, Kuhn et al. (2016) solve a traffic assignment problem with an iterative algorithm. It models the simultaneous movement of network users, assuming travelers follow their shortest paths. Congestion effects are taken into account by a nonlinear relationship between the flow on an edge and the travel time. Until an equilibrium solution is found, each iteration of the algorithm produces a flow and resulting travel times on the edges. To obtain the lower (upper) limit of the travel time interval for each edge we choose the smallest and largest travel times obtained during several stages of the iterative equilibrium algorithm.

For the population we use the distribution of the population described by Kuhn et al. (2016) as nominal values (lower interval limits). We randomly assign integer interval lengths $\left(\delta_{e, 2}\right)$ up to $x \%$ of the respective nominal value. By varying $x$ we obtain several test instances. We call $x$ the population uncertainty.

We choose an appropriate start and end node with an agglomeration of population between them. Fig. 3 shows two exemplary robust efficient paths for the instance with $x=10$ and $\Gamma=(5,5)$. One of the paths goes directly through the area with high population. Here the time objective function has a small value, whereas the number of people exposed to the risk of health damage in case of an accident is relatively high. The other path avoids highly populated areas, which results in a longer travel time.

https://doi.org/10.1016/j.ejor.2017.12.018 


\section{A.1. Combinatorial Optimization with Cardinality-Constrained Uncertainty}

A. Raith et al./European Journal of Operational Research 267 (2018) 628-642

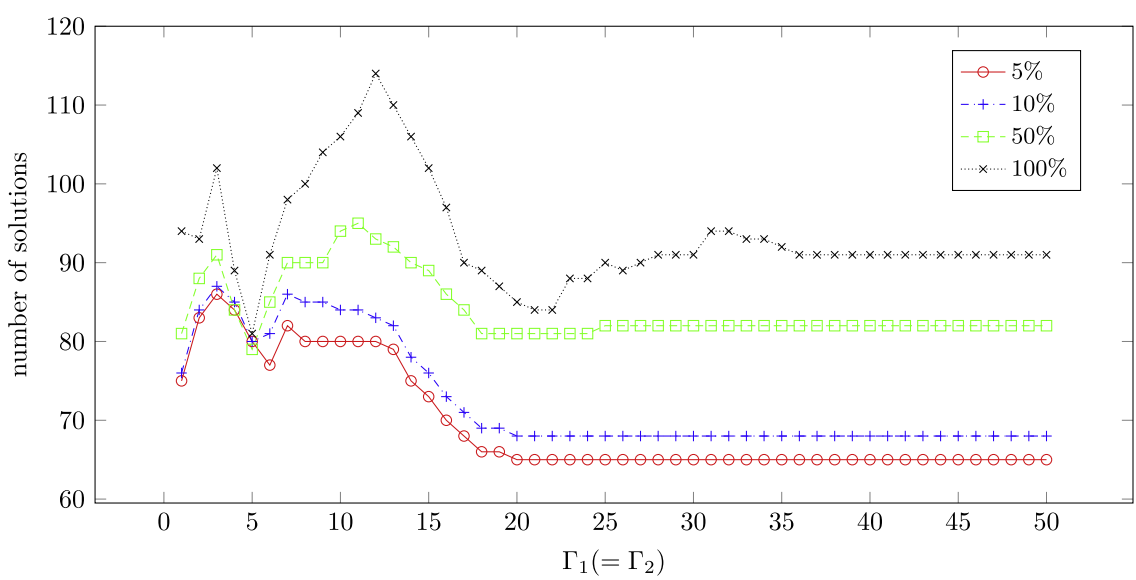

Fig. 4. Number of robust efficient solutions for several values of $\Gamma_{i}$ and population uncertainty $x$.

Table 1

Number of generated solutions for several values of $\Gamma_{i}$ and population uncertainty: Sol $=$ minimal number of robust efficient solutions in a complete set, tDSA = total number of solutions generated in the subproblems, tLSA= total number of solutions found with the multi-objective labeling algorithm (before filtering the robust efficient solutions).

\begin{tabular}{|c|c|c|c|c|c|c|c|c|c|c|c|c|}
\hline \multirow[b]{2}{*}{$\Gamma_{i}$} & \multicolumn{3}{|c|}{ Pop. unc. $\leqq 5 \%$} & \multicolumn{3}{|c|}{ Pop. unc. $\leqq 10 \%$} & \multicolumn{3}{|c|}{ Pop. unc. $\leqq 50 \%$} & \multicolumn{3}{|c|}{ Pop. unc. $\leqq 100 \%$} \\
\hline & Sol & tDSA & tLSA & Sol & tDSA & tLSA & Sol & tDSA & tLSA & Sol & tDSA & tLSA \\
\hline 1 & 75 & 26,288 & 6991 & 76 & 52,887 & 8886 & 81 & 226,008 & 13189 & 94 & 468,828 & 16,768 \\
\hline 2 & 83 & 26,278 & 4529 & 84 & 52,867 & 5879 & 88 & 225,928 & 7830 & 93 & 468,668 & 10,228 \\
\hline 3 & 86 & 26,579 & 2972 & 87 & 53,544 & 3732 & 91 & 229,031 & 4727 & 102 & 475,140 & 5860 \\
\hline 4 & 84 & 26,569 & 1679 & 85 & 53,524 & 2057 & 84 & 228,951 & 2184 & 89 & 474,980 & 2843 \\
\hline 5 & 80 & 26,569 & 691 & 80 & 53,524 & 944 & 79 & 228,951 & 843 & 81 & 474,980 & 940 \\
\hline 10 & 80 & 25,179 & - & 84 & 50,665 & - & 94 & 216,596 & - & 106 & 449,430 & - \\
\hline 20 & 65 & 23,306 & - & 68 & 46,912 & - & 81 & 200,709 & - & 85 & 407,281 & - \\
\hline 30 & 65 & 21,762 & - & 68 & 39,437 & - & 82 & 178,838 & - & 91 & 367,987 & - \\
\hline 40 & 65 & 20,264 & - & 68 & 32,655 & - & 82 & 154,478 & - & 91 & 330,851 & - \\
\hline 50 & 65 & 15,011 & - & 68 & 30,306 & - & 82 & 135,934 & - & 91 & 296,009 & - \\
\hline
\end{tabular}

\subsection{Results}

The algorithms are implemented in $\mathrm{C}++$, compiled under Debian 8.6 with $\mathrm{g}++4.9 .2$ compiler, and run on a Laptop with 2.10 gigahertz quad core processor and 7.71 gigabytes of RAM. If not stated otherwise, we use an implementation of DSA that contains all enhancements described in Section 3.1. In addition, it checks in the beginning, whether the instance has objective-independent element order. If this is the case, we use a special version of DSA, as proposed in Lemma 17, which we will refer to as DSA-oi: instead of the nested for-loops in Lines 3-6 of Algorithm 4 it only contains one for-loop.

For solving the subproblems we use an implementation of the algorithm by Martins (1984) (with the difference that the labels are selected w.r.t. their aggregate cost instead of using the lexicographic order). There and in the implementation of LSA, we additionally delete new labels at any node if they are dominated by an existing label at $t$

In the figures, one data point represents one measurement, except for Section 4.2.3, where we took the average running time of 40 runs.

To compare the performance of our solution approaches, we solve the bi-objective hazardous material transportation instance described above for different values of population uncertainty $x$ and $\boldsymbol{\Gamma}$. We always choose the same value for $\Gamma_{1}$ and $\Gamma_{2}$ and we will refer to this value as $\Gamma_{i}$ in the following. In addition, we compare the performance of the algorithms on an instance with with two correlated objective functions and on an instance with three objectives. We further evaluate the improvement gained by our enhancement of DSA (solution checking). Finally, we generate an instance with objective-independent element order and investigate to which extent the performance time of the DSA benefits from the results in Lemma 17.

4.2.1. Number of robust efficient solutions for the hazardous material transportation instance

Fig. 4 shows the minimal cardinality of a complete set of robust efficient solutions for the generated instances for several values of $x$ and $\Gamma_{i}$. In general, for increasing values of population uncertainty $x$ the number of robust efficient solutions increases as well, because of the higher variation allowed in the second objective. We do not observe a direct dependency on $\Gamma_{i}$, but for values greater than 25 the number of robust efficient solutions stays the same or differs only little. The reason is that the robust efficient solutions contain only between 39 and 56 edges. Furthermore, the interval lengths $\delta_{e, 1}$ resp. $\delta_{e, 2}$ of some edges are 0 . Hence, at some point, allowing more edges to differ from their minimal cost makes no difference.

In Table 1, we present the number of solutions generated in total: For DSA we add the number of solutions obtained by solving the subproblems (which possibly contain identical solutions several times). For LSA we list the number of solutions found by the multi-objective labeling algorithm before the filtering step. The number of solutions generated increases with the population

https://doi.org/10.1016/j.ejor.2017.12.018 


\section{A. Publications}

640

A. Raith et al./European Journal of Operational Research 267 (2018) 628-642

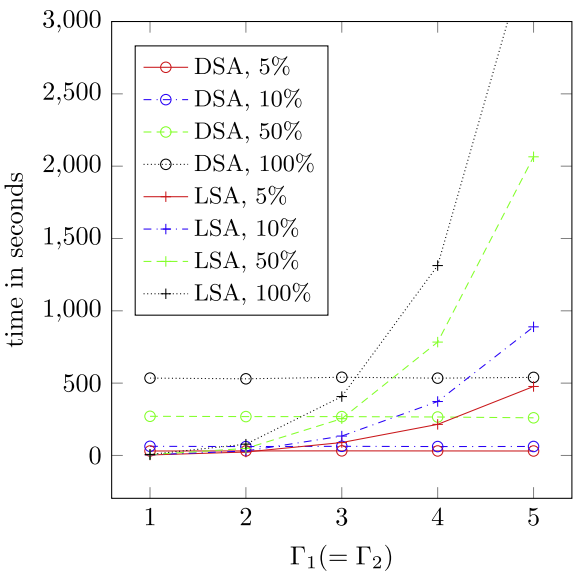

(a) $\Gamma_{1}=\Gamma_{2} \in\{1, \ldots, 5\}$

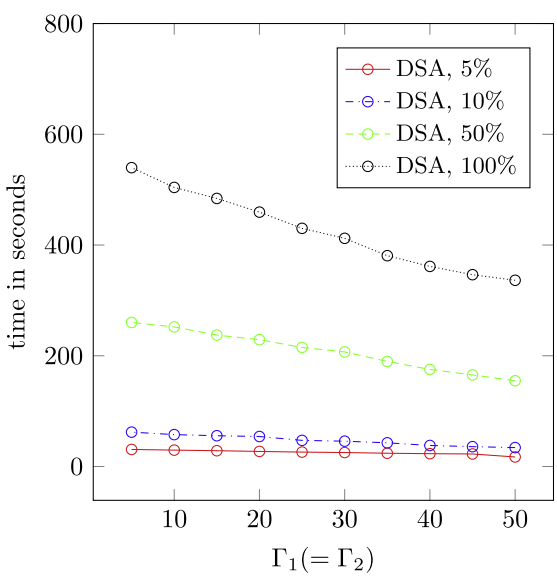

(b) $\Gamma_{1}=\Gamma_{2} \in\{5,10, \ldots, 50\}$

Fig. 5. Running time of DSA and LSA for several values of $\Gamma_{i}$ and population uncertainty $x$ on two different scales.

uncertainty $x$ (as does the number of robust efficient solutions). It tends to decrease for increasing $\Gamma_{i}$ (with a few exceptions). For the DSA that is because of the decreasing number of subproblems solved (see Fig. 8(b)).

\subsubsection{Comparison of the two solution approaches}

Fig. 5 shows the running time of DSA and LSA for several values of $\Gamma_{i}$ and $x$. The running time of LSA increases with $\Gamma_{i}$, whereas the running time of DSA decreases (see also Fig. 8(a)). The reason is that for increasing $\Gamma_{i}$, the number of objectives in the deterministic multi-objective problem solved during LSA increases as well. However, the maximal number of subproblems solved during DSA decreases. For small values of $\Gamma_{i}$ LSA solves the given instances faster, for higher values DSA has a better performance.

Choosing a higher value for $x$ results in a greater maximal and mean deviation from the nominal value and a higher number of different values of $\delta_{e, 2}$. When $x$ is increased, the running time of both algorithms increases. In the case of DSA, this can be explained by the higher number of different values of $\delta_{e, 2}$, which leads to a higher number of subproblems.

\subsubsection{Correlated objective functions}

We additionally generate an instance with two strongly correlated objective functions: we use the travel time as one objective and generate a second travel time objective by multiplying the nominal times and the interval lengths each by a random factor between 0.9 and 1.1 .

Both algorithms benefit a lot from the correlation, all running times are now less than four seconds, as shown in Fig. 6. In comparison, LSA benefits more from correlated objective function values: The values of $\Gamma_{i}$, for which it is still faster than DSA are much higher on this instance than on the original hazardous material transportation instance considered in Section 4.2.2. For small values of $\Gamma_{i}$ it is much faster than DSA.

\subsubsection{Three objectives}

Since we are also interested in the performance of the algorithms for problems with more than two objectives, we generate an artificial third objective: For the nominal values we use again the nominal population. We generate random interval lengths in the same range as the other population objective. That means, the value of population uncertainty in general is the same for both

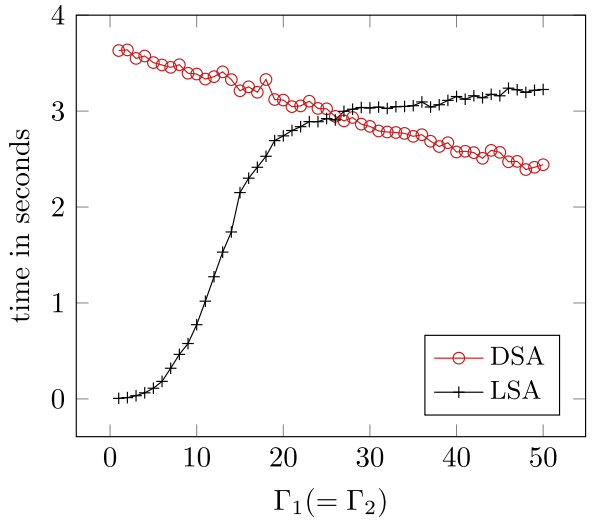

Fig. 6. Running time of DSA and LSA for an instance with two strongly correlated objective functions.

population objectives, but the specific interval lengths of each edge may differ. Because of the identical nominal values, two of the three objectives are correlated. Fig. 7 shows the running times on this instance in comparison to the instance with two objectives described above.

The running time of both algorithms increases by including the additional objective, even though it is strongly correlated to one of the original objectives. The relative difference between the running time of the instance with two objectives and the instance with three objectives increases with $\Gamma_{i}$ for LSA, whereas it decreases for DSA.

4.2.5. Evaluation of the improvement obtained by solution checking

To evaluate the obtained improvement by using solution checking in DSA, we use Algorithm 4 as Step 3 of Algorithm 3. We compare the running time of the version containing solution checking to the running time of the version without this enhancement (Fig. 8(a)). Additionally, we count the solved subproblems (Fig. 8(b)). Where fewer subproblems were solved because of the enhancement, the running times differ significantly, for all other instances they are nearly equal. Hence, the check itself does

https://doi.org/10.1016/j.ejor.2017.12.018 


\section{A.1. Combinatorial Optimization with Cardinality-Constrained Uncertainty}

A. Raith et al./European Journal of Operational Research 267 (2018) 628-642

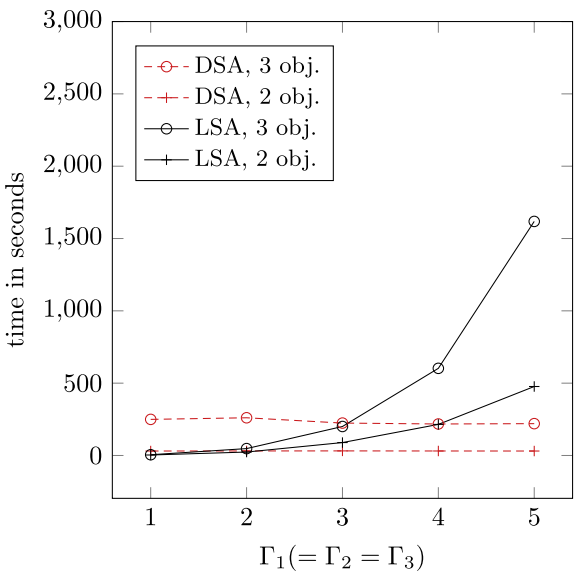

(a) Population uncertainty $5 \%$

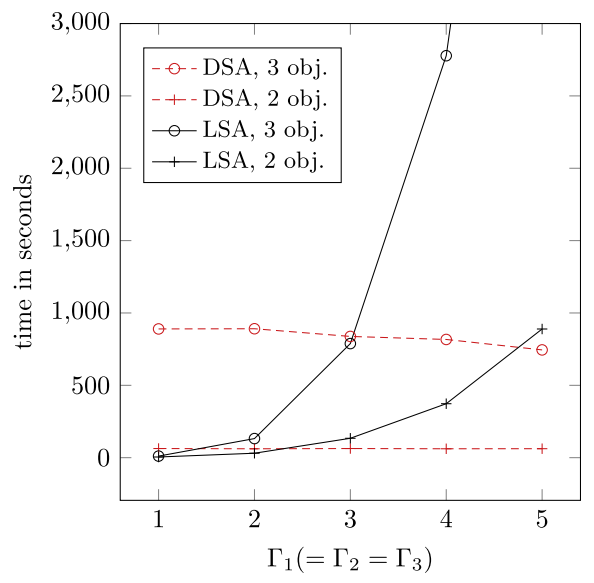

(b) Population uncertainty $10 \%$

Fig. 7. Running time of DSA and LSA for an instance with three objectives and an instance with two objectives.

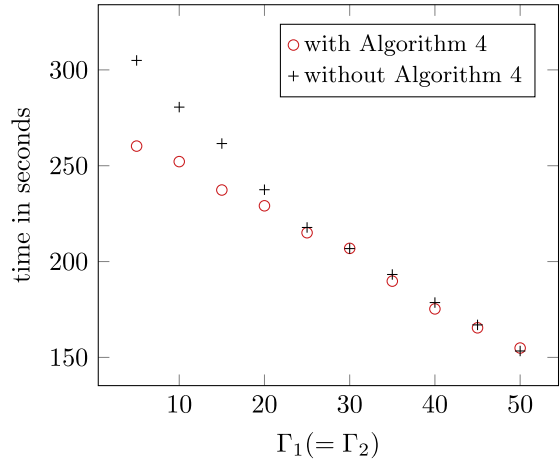

(a) Running time

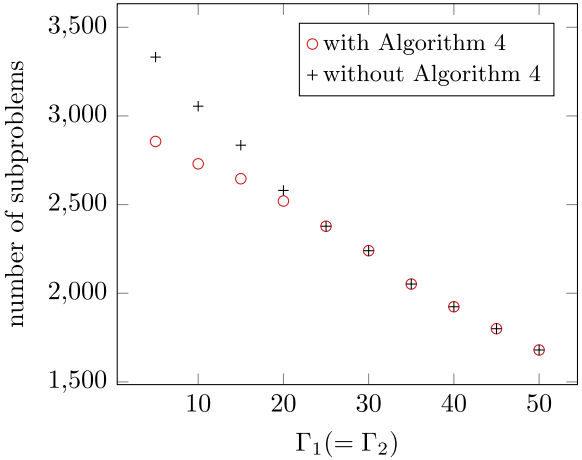

(b) Number of Subproblems.

Fig. 8. Running time and number of solved subproblems of DSA with and without solution checking (population uncertainty $50 \%$ ).

not slow down the algorithm significantly in comparison to the acceleration that we obtain when subproblems can be skipped. We conclude that it is worth using the enhancement, but as $\Gamma_{i}$ increases solution checking becomes less effective.

Note that, since Lemma 13 allows to exclude even more subproblems than excluded in Algorithm 4, further speed-ups may be achieved by implementing a more sophisticated solution checking. However, already when using Algorithm 4, the benefit of solution checking is clearly visible.

\subsubsection{Evaluation of DSA for instances with objective-independent element order}

For instances with objective-independent element order, we use the special version DSA-oi as proposed in Lemma 17. To compare its performance to the general version of DSA we construct an instance with objective-independent element order: instead of generating interval lengths for the population objective we use the interval lengths of the travel time objective. Fig. 9 shows that DSA-oi has a much better performance than the general algorithm. The test, whether the instance is objective-independent, only takes a small fraction of the running time (for our instances $1.4 \cdot 10^{-5}$ seconds). Therefore, it is reasonable to check each instance for objective-independent element order before solving it with DSA.

\section{Conclusion}

In this paper we developed two approaches to find minmax robust solutions for multi-objective combinatorial optimization problems with cardinality-constrained uncertainty. We extended an algorithm by Bertsimas and $\operatorname{Sim}$ (2003) to multi-objective optimization (DSA), suggested an enhancement and developed a special version for instances with objective-independent element order. We also introduced a second approach and used it to develop a label setting algorithm (LSA) for the multi-objective uncertain shortest path problem.

We tested our algorithms on several instances of the multiobjective uncertain shortest path problem arising from hazardous material transportation. On most of the tested instances DSA has a better performance, but LSA is faster for small values of $\Gamma_{i}$. If the two objective functions are strongly correlated, LSA is competitive even for higher values of $\Gamma_{i}$. This appears often in shortest path problems, where, e.g., the distance, travel time and fuel consumption are correlated.

When implementing DSA we recommend to use the proposed enhancements and to check whether the special version for instances with (partial) objective-independent element order can be used. The checks do not take long in comparison to the total

https://doi.org/10.1016/j.ejor.2017.12.018 


\section{A. Publications}

642

A. Raith et al./European Journal of Operational Research 267 (2018) 628-642

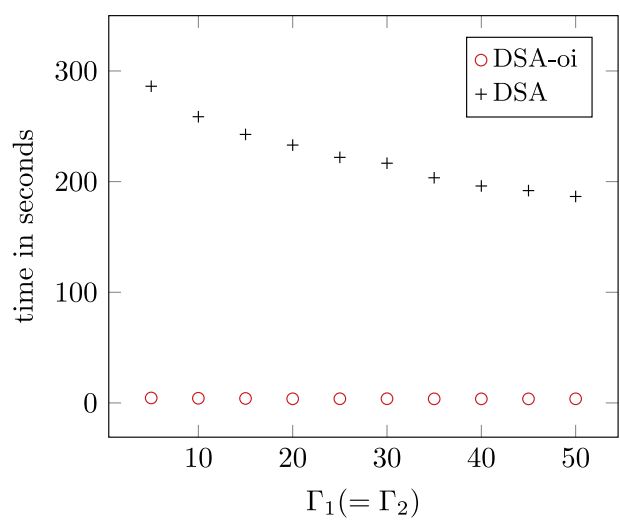

Fig. 9. Comparison of DSA and DSA-oi for instances with objective-independent element order.

running time, and if their result is positive, the algorithm can be accelerated significantly.

For further investigations other variants of multi-objective cardinality-constrained uncertainty are of interest. A second way to extend the single-objective concept is to require the edges whose costs differ from their minimal values to be the same for all objectives. In this case the uncertainties in the objectives are no longer independent of each other and using point-based or set-based minmax robust efficiency leads to different solution sets. An interesting variation of cardinality-constrained uncertainty is not to consider a bound on the cardinality, but on the sum of the deviation from their minimal values.

Further research on robust multi-objective optimization includes other types of uncertainty, e.g., discrete scenario sets or polyhedral or ellipsoidal uncertainty. Also the case of decision uncertainty, in which the solution found cannot be realized exactly, is of interest, see Eichfelder, Krüger, and Schöbel (2017) for first results.

The algorithms for the multi-objective cardinality-constrained uncertain shortest path problem presented in this paper can easily be extended to the multi-objective single-source shortest path problem. There, a complete set of efficient paths from a start node $s$ to all other nodes is to be found. In the deterministic case, there exist algorithms (e.g. the algorithm by Martins, 1984) for which it can be shown that the running time is polynomial in the output size. It would be interesting to investigate whether this is the case for the uncertain problem, too.

\section{Acknowledgment}

Lisa Thom was supported by DFG RTG 1703 "Resource Efficiency in Interorganizational Networks".

\section{References}

Aissi, H., Bazgan, C., \& Vanderpooten, D. (2009). Min-max and min-max regret versions of combinatorial optimization problems: A survey. European Journal of Operational Research, 197(2), 427-438.

Bar-Gera, H., Kwon, C., Li, J., \& Stabler, B. Transportation Networks for Research Core Team. Transportation Networks for Research. https://github.com/bstabler/ TransportationNetworks. Accessed: November, 04, 2016

Ben-Tal, A., El Ghaoui, L., \& Nemirovski, A. (2009). Robust optimization. Princeton University Press.

Bertsimas, D., \& Sim, M. (2003). Robust discrete optimization and network flows. Mathematical Programming, 98(1), 49-71.

Bertsimas, D., \& Sim, M. (2004). The price of robustness. Operations Research, 52(1),

Chuong, T. D. (2016). Optimality and duality for robust multiobjective optimization problems. Nonlinear Analysis: Theory, Methods \& Applications, 134, 127-143.

Doolittle, E Kerivin, H. M. \& Wiecek. M. M. (2012) A robust multiobjective optimization problem with application to Internet routing. Technical report R2012-11-DKW. Clemson University.

Ehrgott. M. (2006). Multicriteria optimization. Berlin, Heidelberg: Springer.

Ehrgott, M., Ide, J., \& Schöbel, A. (2014). Minmax robustness for multi-objective optimization problems. European Journal of Operational Research, 239, 17-31

Eichfelder, G., Krüger, C., \& Schöbel, A. (2017). Decision uncertainty in multiobjective optimization. Journal of Global Optimization, 69, 485-510.

Erkut, E. Tjandra, S. A., \& Verter, V. (2007). Hazardous materials transportation. Handbooks in Operations Research and Management Science, 14, 539-621.

Fliege, J., \& Werner, R. (2014). Robust multiobjective optimization \& applications in portfolio optimization. European Journal of Operational Research, 234(2), $422-433$

Gandibleux, X., Beugnies, F., \& Randriamasy, S. (2006). Martins' algorithm revisite for multi-objective shortest path problems with a maxmin cost function. $40 R$ $4(1), 47-59$.

Gorski, J. Klamroth, K. \& Ruzika, S. (2012). Generalized multiple objective bottleneck problems. Operations Research Letters, 40(4), 276-281.

Hassanzadeh, F., Nemati, H., \& Sun, M. (2013). Robust optimization for multiobjective programming problems with imprecise information. Procedia Computer Science, 17, 357-364.

Ide, J., Köbis, E., Kuroiwa, D., Schöbel, A., \& Tammer, C. (2014). The relationship between multi-objective robustness concepts and set valued optimization. Fixe Point Theory and Applications, 2014(83), 1-20. doi:10.1186/1687-1812-2014-83.

de, J., \& Schöbel, A. (2016). Robustness for uncertain multi-objective optimization: A survey and analysis of different concepts. OR Spectrum, 38(1), 235-271.

de, J., Tiedemann, M., Westphal, S., \& Haiduk, F. (2015). An application of deterministic and robust optimization in the wood cutting industry. 4OR, 13(1), 35-57.

ori, M., Martello, S., \& Pretolani, D. (2010). An aggregate label setting policy for the multi-objective shortest path problem. European Journal of Operational Research, 207(3), 1489-1496

Kalantari, M., Dong, C., \& Davies, I. J. (2016). Multi-objective robust optimisation of unidirectional carbon/glass fibre reinforced hybrid composites under flexura loading. Composite Structures, 138, 264-275.

Kuhn, K., Raith, A., Schmidt, M., \& Schöbel, A. (2016). Bicriteria robust optimization European Journal of Operational Research, 252, 418-431.

Kuroiwa, D., \& Lee, G. M. (2012). On robust multiobjective optimization. Vietnam Journal of Mathematics, 40(2-3), 305-317.

Lee, T., \& Kwon, C. (2014). A short note on the robust combinatorial optimization problems with cardinality constrained uncertainty. 4OR, 12(4), 373-378.

Martins, E. Q. V. (1984). On a multicriteria shortest path problem. European Journa of Operational Research, 16(2), 236-245.

Park, K.-C., \& Lee, K.-S. (2007). A note on robust combinatorial optimization problem. Management Science and Financial Engineering, 13(1), 115-119.

Soyster, A. L. (1973). Convex programming with set-inclusive constraints and applications to inexact linear programming. Operations Research, 21(5), 1154-1157.

wiecek, M. M., \& Dranichak, G. M. (2016). Robust multiobjective optimization for decision making under uncertainty and conflict. In Optimization challenges in complex, networked and risky systems (pp. 84-114). INFORMS.

Yu, H., \& Liu, H. M. (2013). Robust multiple objective game theory. Journal of Optimization Theory and Applications, 159(1), 272-280.

https://doi.org/10.1016/j.ejor.2017.12.018 


\section{A.2. Extensions of Labeling Algorithms for Multi-Objective Uncertain Shortest Path Problems}

published in Networks (Raith et al., 2018a)

Authors: Andrea Raith, Marie Schmidt, Anita Schöbel, Lisa Thom 


\title{
Extensions of labeling algorithms for multi-objective uncertain shortest path problems
}

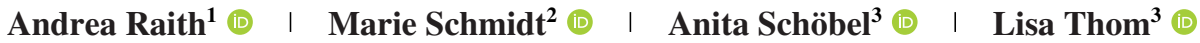 \\ ${ }^{1}$ Department of Engineering Science, The University of Auckland, Auckland 1142, New Zealand \\ ${ }^{2}$ Department of Technology and Operations Management, Rotterdam School of Management, Erasmus University Rotterdam, 3000 DR Rotterdam, \\ The Netherlands \\ ${ }^{3}$ Institut für Numerische und Angewandte Mathematik, Georg-August-Universität Göttingen, Göttingen 37083, Germany
}

\section{Correspondence}

Marie Schmidt, Department of Technology and Operations Management, RSM, Erasmus University Rotterdam, PO Box 1738, 3000 DR Rotterdam, The Netherlands.

Email: schmidt2@rsm.nl

\section{Funding information}

Deutsche Forschungsgesellschaft (DFG), Grant/Award Number: 172586392

\begin{abstract}
We consider multi-objective shortest path problems in which the edge lengths are uncertain. Different concepts for finding so-called robust efficient solutions for multi-objective robust optimization exist. In this article, we consider multi-scenario efficiency, flimsily and highly robust efficiency, and point-based and set-based minmax robust efficiency. Labeling algorithms are an important class of algorithms for multi-objective (deterministic) shortest path problems. We analyze why it is, for most of the considered concepts, not straightforward to use labeling algorithms to find robust efficient solutions. We then show two approaches to extend a generic multiobjective label correcting algorithm for these cases. We finally present extensive numerical results on the performance of the proposed algorithms.
\end{abstract}

\section{KEYWORDS}

finite uncertainty, label correcting algorithm, multi-objective optimization, multi-objective robust optimization, robust optimization, shortest path problem

\section{1 | INTRODUCTION}

We consider the well-known shortest path problem in terms of the recent field of multi-objective robust optimization, which combines concepts of multi-objective optimization and robust optimization.

In multi-objective optimization, several (conflicting) objectives are optimized simultaneously. For example, when transporting hazardous material, one wants to minimize the travel time, the expenses and the risk for the environment and the inhabitants of the region at the same time. In multi-objective optimization one usually tries to find (Pareto) efficient solutions, which cannot be improved in one objective without worsening them in another objective.

Robust optimization is one approach to deal with uncertain parameters. In particular in practical applications, usually not all parameters of an optimization problem are reliably predictable. The travel time in a road network, for example, depends on the traffic congestion and the weather. In robust optimization one wants to hedge against (all) possible scenarios, for example, by considering the worst case for each solution.

During the last decade, concepts of multi-objective and robust optimization have been combined to multi-objective robust optimization, where multiple objectives with uncertain parameters are considered. Several concepts to define robust efficient 
solutions have been developed; for a recent overview see [24, 41]. In this article, we consider five different concepts of robust efficiency: A solution is multi-scenario efficient $[7,18]$ if it cannot be improved for one scenario without worsening it in another scenario. Flimsily (resp. highly) robust efficient solutions [24] are efficient for at least one scenario (resp. for all scenarios). Point-based [27] and set-based [17] robust efficiency generalize the single-objective concept of minmax robustness, where the worst case for each solution is considered. The concepts can also be generalized to other cones than the standard Pareto cone [23].

The shortest path problem has been extensively investigated, both in terms of multi-objective and robust optimization, but has so far received only little attention in the context of multi-objective robust optimization. A fast way to solve the singleobjective deterministic shortest path problem is to use a labeling algorithm, for example, the label setting algorithm of Dijkstra [14] or the label correcting algorithm of Bellman and Ford [2]. Label setting algorithms can be used for nonnegative edge costs, whereas label correcting algorithms are also suitable for negative edge costs. Labeling algorithms have been generalized successfully to multi-objective optimization, for example, in [28] for nonnegative edge costs and in [12] for general edge costs. For an overview on multi-objective labeling algorithms and a computational study see [33].

In robust optimization the considered uncertainty set plays an important role. The edge costs can, for example, all be influenced by the same parameter, as public events or weather conditions influence the travel time on all roads in an area. They can also vary independently of each other, as traffic lights slow down the passing through each road segment individually. In this article, we consider a finite set of possible scenarios, which affect the costs of all edges. The robust shortest path problem with a finite scenario set has first been investigated in [42] for two different robustness concepts. The authors present a pseudopolynomial algorithm, which is an extended labeling algorithm. For one of the robustness concepts, the robust shortest path problem reduces to a minmax shortest path problem, which has earlier been considered in other contexts, see [32]. Reference [15] compare the minmax robust solutions for different assumed uncertainty sets based on a discrete sample of scenarios. Other popular robustness concepts include deviation robustness (see, eg, $[10,30,34]$ for results on the shortest path problem). Reference [13] uses a concept similar to Pareto efficiency to solve robust shortest path problems. For an overview on solution approaches for the robust shortest path problem with various robustness concepts and uncertainty sets, see, for example, $[1,19,25]$

The multi-objective robust shortest path problem has only been considered in few papers so far. The authors of [26] introduce a solution algorithm for combinatorial problems with two objectives, of which only one is uncertain. They assume discrete and polyhedral uncertainty sets. Combinatorial problems with so-called cardinality-constrained uncertainty, an uncertainty concept first introduced in [4] for single-objective problems, are considered in [38].

The remainder of this article is structured as follows: First, we give an introduction to the multi-objective shortest path problem with uncertain edge costs and present several popular concepts of robust efficiency in Section 2. In Section 3, we state conditions under which a generic multi-objective label correcting algorithm can be used to find robust efficient solutions for the multi-objective uncertain problem. In Section 4, we investigate for each of the introduced concepts of robust efficiency whether they satisfy these conditions. In case the conditions are not satisfied, we propose algorithms to find robust efficient solutions. They either extend the algorithm from Section 3 or split the problem into subproblems, which can be solved by a repeated application of this algorithm. We experimentally test and compare the developed algorithms in Section 5.

\section{2 | MULTI-OBJECTIVE ROBUST SHORTEST PATH PROBLEMS}

We first give an introduction to the multi-objective shortest path problem following [16].

Let a digraph $G=(V, E)$ with node set $V$ and set of directed edges $E$, a start node $s \in V$ and a target node $t \in V$ be given. We assume that no parallel edges exist, that is, an edge $e$ is uniquely defined by its start node $v$ and end node $v^{\prime}$ and can be written as $e=\left(v, v^{\prime}\right)$. A path is a chain of adjacent edges in $G$. We say that a node $v$ lies on a path if $v$ is start or end node of one of its contained edges. A path is simple if it contains each node at most once. For each node $v \in V$ let $\mathcal{Q}^{v}$ be the set of all simple paths in $G$ from $s$ to $v$. For a simple path $q$ and two nodes $v, v^{\prime}$ on $q$ we denote the subpath of $q$ from $v$ to $v^{\prime}$ by $q_{v, v^{\prime}}$.

Further, let a multi-objective cost function $c: E \rightarrow \mathbb{R}^{k}$ on the edges be given, that is, $c$ assigns a cost vector to each edge $e \in E$. The cost $z(q)$ of a path $q$ is the sum of the costs of the edges it traverses, that is, for a simple path $q$ we have $z(q)=\sum_{e \in q} c(e)$. Two paths $q, q^{\prime}$ are called equivalent if they have the same start and end node and $z(q)=z\left(q^{\prime}\right)$. Given an instance $(G, c, s, t)$ we define the multi-objective shortest path problem as

https://doi.org/10.1002/net.21815 


$$
(\operatorname{MOSP}) \quad \min _{q \in \mathcal{Q}^{t}} z(q)=\left(\begin{array}{c}
z_{1}(q) \\
\vdots \\
z_{k}(q)
\end{array}\right) .
$$

A solution that minimizes all objectives simultaneously does usually not exist. We hence have to explain what "min" means: We introduce the well-known concept of efficient solutions.

Notation 1. For two vectors $y^{1}, y^{2} \in \mathbb{R}^{k}$ we use the notation

$$
\begin{aligned}
& y^{1} \leq y^{2} \Leftrightarrow y_{i}^{1} \leqq y_{i}^{2} \text { for } i=1, \ldots, k \text { and } y^{1} \neq y^{2}, \\
& y^{1} \leqq y^{2} \Leftrightarrow y_{i}^{1} \leqq y_{i}^{2} \text { for } i=1, \ldots, k .
\end{aligned}
$$

Furthermore, we use $\mathbb{R}_{\geq}^{k}=\left\{y \in \mathbb{R}^{k}: 0 \leq y\right\}$.

In the following, we only use the symbols $<$ (strictly less than) and $\leqq$ (less than or equal to) to compare scalars.

Definition 2. A path $q^{\prime}$ dominates another path $q$ with the same start and end node if $z\left(q^{\prime}\right) \leq z(q)$. We also say that $z\left(q^{\prime}\right)$ dominates $z(q)$. A path $q \in \mathcal{Q}^{t}$ is an efficient path for (MOSP) if there is no $q^{\prime} \in \mathcal{Q}^{t}$ such that $z\left(q^{\prime}\right)$ dominates $z(q)$. Then $z(q)$ is called non-dominated. A complete set of efficient paths is a set $\mathcal{Q}^{\prime} \subseteq \mathcal{Q}^{t}$, such that for each efficient path $q \in \mathcal{Q}^{t}$ there exists an equivalent path $q^{\prime} \in \mathcal{Q}^{\prime}$.

Solving $(M O S P)$ means to find a complete set of efficient paths.

Often the costs for the edges are not known exactly, but they depend on the scenario that occurs, for example, travel times can depend on the time of the day, on special events, on the weather, etc. Here, we consider multi-objective uncertain shortest path problems with a finite set of scenarios $\mathcal{U}:=\left\{\xi_{1}, \ldots, \xi_{r}\right\}$. In multi-objective uncertain optimization, the cost vectors depend on the scenario which occurs, that is, for every scenario we may get a different cost vector. Hence, $c$ is a function that assigns a cost vector $c(e, \xi)=\left(c_{1}(e, \xi), \ldots, c_{k}(e, \xi)\right)^{T} \in \mathbb{R}^{k}$ to each edge $e \in E$ for each scenario $\xi \in \mathcal{U}$. We hence obtain a cost matrix

$$
c(e):=\left(\begin{array}{ccc}
c_{1}\left(e, \xi_{1}\right) & \ldots & c_{1}\left(e, \xi_{r}\right) \\
\vdots & & \\
c_{k}\left(e, \xi_{1}\right) & \ldots & c_{k}\left(e, \xi_{r}\right)
\end{array}\right)
$$

for every edge $e$. The cost of a path $q$ is the sum of the costs of the edges it traverses, that is, for a simple path we have $z(q, \xi)=\sum_{e \in q} c(e, \xi)$ and its cost matrix is $z(q)=\sum_{e \in q} c(e)$. In this setting, two paths are called equivalent if they have the same start and end node and $z(q)=z\left(q^{\prime}\right)$. For a matrix $Y$ we denote by $Y_{(i, \cdot)}$ its $i$-th row and by $Y_{(\cdot, j)}$ its $j$-th column, that is, $c(e)_{(\cdot, j)}=c\left(e, \xi_{j}\right)$.

The multi-objective uncertain shortest path problem (MOUSP) is the family of multi-objective optimization problems

$$
(\operatorname{MOUSP}) \quad\left(\left(M O S P_{\xi}\right) \min _{q \in Q^{t}} z(q, \xi), \xi \in \mathcal{U}\right)
$$

The notion of what is a good solution to a multi-objective uncertain problem is not trivial. In multi-objective robust optimization one searches for so-called robust efficient solutions. We now present some concepts to define robust efficient solutions proposed in the literature.

The concept of multi-scenario efficiency $[7,18]$ applies the idea of efficiency to several scenarios and multiple objective functions at the same time: A solution is multi-scenario efficient, if there is no other solution which dominates it in one scenario and is as least as good in all other scenarios.

https://doi.org/10.1002/net.21815 


\section{${ }^{4} \perp$ WILEY}

RAITH ET AL.

Definition 3 ([7, 18]). A solution $q \in \mathcal{Q}^{t}$ is multi-scenario efficient for (MOUSP) if

$$
\nexists q^{\prime} \in \mathcal{Q}^{t}:\left(\begin{array}{c}
z_{1}\left(q^{\prime}, \xi_{1}\right) \\
\vdots \\
z_{k}\left(q^{\prime}, \xi_{1}\right) \\
z_{1}\left(q^{\prime}, \xi_{2}\right) \\
\vdots \\
z_{k}\left(q^{\prime}, \xi_{r}\right)
\end{array}\right) \leq\left(\begin{array}{c}
z_{1}\left(q, \xi_{1}\right) \\
\vdots \\
z_{k}\left(q, \xi_{1}\right) \\
z_{1}\left(q, \xi_{2}\right) \\
\vdots \\
z_{k}\left(q, \xi_{r}\right) .
\end{array}\right)
$$

Using the concept of highly robust efficiency [5, 24], we look for solutions, which are efficient for every scenario.

Definition 4 ([5, 24]). A solution $q \in \mathcal{Q}^{t}$ is highly robust efficient for (MOUSP) if

$$
\forall \xi \in \mathcal{U}: \nexists q^{\prime} \in \mathcal{Q}^{t}: z\left(q^{\prime}, \xi\right) \leq z(q, \xi)
$$

However, there is no guarantee that a highly robust efficient solution exists. A reasonable condition for a good solution would then be that it should be efficient for at least one of the scenarios. This is called flimsily robust efficiency in [24].

Definition $5([5,24])$. A solution $q \in \mathcal{Q}^{t}$ is flimsily robust efficient for (MOUSP) if

$$
\exists \xi \in \mathcal{U}: \nexists q^{\prime} \in \mathcal{Q}^{t}: z\left(q^{\prime}, \xi\right) \leq z(q, \xi) .
$$

Often in robust optimization one wants to hedge against the worst case. The aim of single-objective minmax robust optimization is to find a solution with the smallest cost in the worst case. We present two generalizations of this concept to multi-objective optimization, point-based and set-based minmax robust efficiency.

Definition 6 ([27]) A solution $q \in \mathcal{Q}^{t}$ is point-based minmax robust efficient for (MOUSP) if it is efficient for the deterministic multi-objective problem

$$
\left(\operatorname{MOSP}_{\max }\right) \quad \min _{q \in Q^{t}}\left(\begin{array}{c}
\max _{\xi \in \mathcal{U}} z_{1}(q, \xi) \\
\vdots \\
\max _{\xi \in \mathcal{U}} z_{k}(q, \xi) .
\end{array}\right)
$$

Definition 7 ([17]). A solution $q \in \mathcal{Q}^{t}$ is set-based minmax robust efficient for (MOUSP) if there is no feasible solution $q^{\prime} \in \mathcal{Q}^{t}$ with

$$
z_{\mathcal{u}}\left(q^{\prime}\right) \subseteq z_{\mathcal{U}}(q)-\mathbb{R}_{\geq}^{k}
$$

where

$$
z_{\mathcal{U}}(q):=\{z(q, \xi): \xi \in \mathcal{U}\}
$$

We remark that more concepts for defining robust efficient solutions to a multi-objective uncertain optimization problem exist; we refer to $[24,41]$ for an overview.

In this article, we are interested in solving the multi-objective robust shortest path problem with $k$ objective functions for a finite scenario set $\mathcal{U}$ with $|\mathcal{U}|=r$. An instance is hence given as $(G, \mathcal{U}, c, s, t)$ with $G$ the digraph,

https://doi.org/10.1002/net.21815 
RAITH ET AL.

$s$ the start and $t$ the end node, and $c$ denoting the objective function, which assigns for each scenario a cost vector $c(e, \xi)$ to each edge.

(MORSP $\quad$ Given a concept of robust efficiency, find a complete set of robust efficient solutions for (MOUSP).

That is, find a complete set of multi-scenario efficient, flimsily robust efficient, highly robust efficient, point-based minmax robust efficient or set-based minmax robust efficient solutions.

For $|\mathcal{U}|=1,(M O U S P)$ reduces to $(M O S P)$. In this case, the robust efficient solutions w.r.t. any of the concepts defined in this section are exactly the efficient solutions of $(M O S P)$.

\section{GENERAL LABEL CORRECTING ALGORITHM}

Labeling algorithms are a standard method for solving shortest path problems, in the single-objective as well as in the multiobjective case. Label setting algorithms can be used for instances with positive edge costs, whereas label correcting algorithms also work for negative edge costs, as long as there are no negative cycles. They can be based on node selection or label selection. We consider a generic label selection method as given in [20] for the multi-objective shortest path problem (see also [9] for the bi-objective problem)

A label is a tuple $l=\left(v, z, l^{\prime}\right)$ consisting of

- a node $v \in V$ (we say that $l$ is a label at $v$ ),

- a cost $z(l)$, and

- a predecessor label $l^{\prime}$ (or 0 if $l$ is the start label with cost 0 at $s$ ).

Every label $l$ at a node $v \neq s$ represents a path $q$ from $s$ to $v$. That means that $z(l)=z(q)$ and $l$ 's predecessor label $l^{\prime}$ represents the subpath of $q$ from $s$ to $v^{\prime}$, with $\left(v^{\prime}, v\right)$ being the last edge of $q$. Given the label $l$, its corresponding path $q$ can be constructed by backtracking the nodes of the predecessor labels. These labels are called ancestors of $l$.

The labels are constructed iteratively from their predecessor labels. We store them in two label sets: A newly created label is first added to the set of temporary labels $\mathcal{T}$. As soon as a label $l \in \mathcal{T}$ at a node $v$ is chosen in the label selection step, it is stored in the label set $\mathcal{L}$ instead and, at the end nodes of all outgoing edges of $v$, new labels with predecessor label $l$ are created. The cost of a label can efficiently be computed by adding the cost of the predecessor label and the edge cost. We say that a label $l$ is dominated by a label $l^{\prime}$ if $z(l)$ is dominated by $z\left(l^{\prime}\right)$.

Algorithm 1 is a generic label correcting algorithm with label selection as given in [20], but with an adjustment: We look for a complete set and not for the whole set of efficient solutions as done in [20]. This is why we only keep newly created labels if there is not yet any other label at the same node with the same cost. That is, we only keep track of a new path if it is not equivalent to an already existing path. Label correcting algorithms are widely used for solving multi-objective shortest path problems. The goal of this article is to make use of labeling algorithms also for solving uncertain multi-objective shortest path problems, that is, to compute robust efficient shortest paths.

We now discuss how we can transfer Algorithm 1 to a solution algorithm for solving the multi-objective robust shortest path problem. The first difference is that in the concepts of robust efficiency given in Section 2, the set of optimal solutions, that is, the set of robust efficient paths in $\mathcal{Q}^{t}$, is defined explicitly and not implicitly via a dominance relation. However, in order to compare label costs we need a suitable definition of dominance. For the decision if a path dominates another one, all data of the uncertain problem has to be available, that is, we need cost matrices $c(e)$ given in (1) on every edge $e \in E$. Finally, Algorithm 1 can only work if Bellman's principle of optimality [2] holds for the given concept of robust efficiency.

We summarize these conditions below.

1. Principle of optimality: For every instance $(G, \mathcal{U}, c, s, t)$ of $(M O U S P)$ we require: If $q \in \mathcal{Q}^{t}$ is a robust efficient path for $(G, \mathcal{U}, c, s, t)$, then for every node $v$ in $q$ the subpath $q_{s, v}$ is robust efficient for the instance $(G, \mathcal{U}, c, s, v)$.

2. For every $k, r \in \mathbb{N}$ there exists a binary (dominance) relation $R \subseteq \mathbb{R}^{k \times r} \times \mathbb{R}^{k \times r}$ with the following properties:

https://doi.org/10.1002/net.21815 
${ }^{6}$-WI LEY RAITH Et AL.

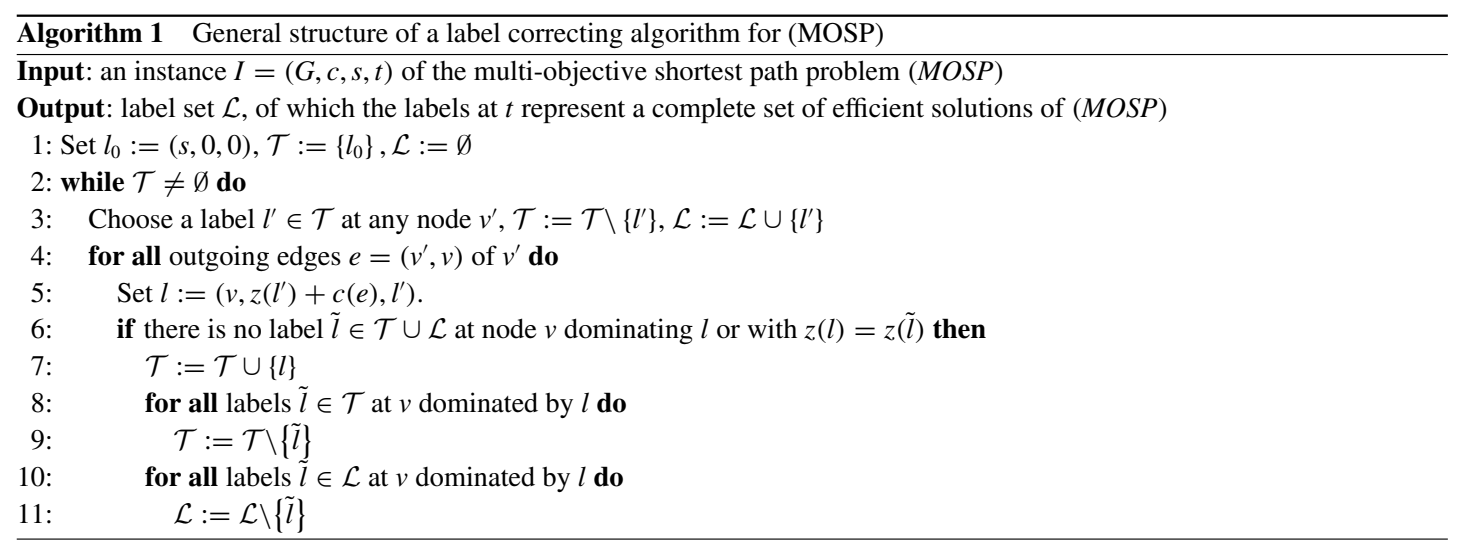

(a) The relation is consistent with the concept of robust efficiency: For all instances with $k$ objectives and $|\mathcal{U}|=r$ :

$$
q \in Q^{t} \text { is robust efficient } \Leftrightarrow \nexists \mathrm{q}^{\prime} \in \mathrm{Q}^{\mathrm{t}}:\left(\mathrm{z}\left(\mathrm{q}^{\prime}\right), \mathrm{z}(\mathrm{q})\right) \in \mathrm{R}
$$

(b) Domination property (see [3]): For all instances with $k$ objectives and $|\mathcal{U}|=r$ :

$$
q \in Q^{t} \text { is not robust efficient } \Rightarrow \exists \text { robust efficient } \mathrm{q}^{\prime} \in \mathrm{Q}^{\mathrm{t}}:\left(\mathrm{z}\left(\mathrm{q}^{\prime}\right), \mathrm{z}(\mathrm{q})\right) \in \mathrm{R}
$$

(c) $R$ is transitive, that is, $\left(Y^{1}, Y^{2}\right) \in R,\left(Y^{2}, Y^{3}\right) \in R \Rightarrow\left(Y^{1}, Y^{3}\right) \in R$.

We say that $q^{\prime}$ dominates $q$ if $\left(z\left(q^{\prime}\right), z(q)\right) \in R$.

With these conditions satisfied, all structural requirements that ensured correctness of Algorithm 1 for the deterministic case are guaranteed and we easily transfer Algorithm 1 to a solution algorithm for solving the multi-objective robust shortest path problem, which we call Algorithm 1'. As input it takes an instance $(G, \mathcal{U}, c, s, t)$ of $(M O R S P)$ with edge costs $c(e) \in \mathbb{R}^{k \times r}$. It executes the same steps as Algorithm 1, but using the definition of dominance given in Condition 2.

To ensure that Algorithm 1' terminates we use the common requirement that the instance is conservative w.r.t. R, that is, for all cycles $C \in G$ either $z(C)=0$ or $\forall Y \in \mathbb{R}^{k \times r}:(Y, Y+z(C)) \in R$. Note that in single-objective deterministic optimization, conservativeness requires that no cycles of negative cost exist.

Theorem 8. If the concept of robust efficiency satisfies Conditions 1 and 2 and the instance is conservative w.r.t. $R$, Algorithm l' finds a complete set of robust efficient solutions.

Proof. We now check that Conditions 1 and 2 and the requirement of conservativeness are indeed enough to guarantee finiteness and correctness of Algorithm 1', proceeding analogously to a proof for correctness of Algorithm 1 in the deterministic case:

We first remark that a label representing a non-simple path $p$ will never be added to $\mathcal{T}$ in Line 6: Whenever the algorithm considers adding the label corresponding to $p$, this label will be dominated by or have the same cost as the label $l^{\prime}$ of the corresponding simple path $p^{\prime}$. Since $p^{\prime}$ is a subpath of $p$ and $R$ is transitive, either $l^{\prime}$ or a label that has the same cost as $l^{\prime}$ or dominates $l^{\prime}$ (and thus $l$ ) will already be contained in $\mathcal{T} \cup \mathcal{L}$. Since in each iteration of Line 3 at least one label is removed from $\mathcal{T}$ and there are only finitely many simple paths in $G$, Algorithm 1' stops after finitely many iterations.

To see that for each robust efficient path $p$ there will be a label $l$ with $z(l)=z(p)$ in $\mathcal{L}$ when the algorithm terminates, note that Lines $1-5$ and 7 describe a routine which iteratively constructs all paths from the source. This routine is complemented by Lines 6, 8-11 in which dominated labels are removed. This also prevents paths with dominated subpaths to be constructed. However, Condition 2(a) guarantees that a label corresponding to a subpath

https://doi.org/10.1002/net.21815 
of a robust efficient path is only removed during the dominance check if there already exists a label with the same cost. Hence for every robust efficient path $p$ a label $l$ with $z(l)=z(p)$ will be found. On the other hand, any label corresponding to a path which is not robust efficient will be sorted out due to Condition 2(b), so that we obtain a complete set of robust efficient paths.

Conditions similar to Conditions 1 and 2 are used in [35] for a labeling approach in cycle-free graphs and (partly) in earlier dynamic programming literature (eg, $[8,21,29,31])$. The main conceptual difference is that they start with a given dominance relation and define optimality and a counterpart to Condition 1 based on this relation. We chose to state the principle of optimality in a way which does not pre-suppose the existence of a suitable dominance relation, since the concepts for robust efficiency studied in this article are not defined via a dominance relation, and it is not immediately obvious for which of the concepts a suitable dominance relation exists (see Section 4 for the corresponding analysis).

Further, instead of requiring Property 2(b), they often require asymmetry of the considered relation. Although on their own these properties are not equivalent, they are equivalent if Properties 2(a) and 2(c) hold, as we show in the following lemma.

Lemma 9. Let $R$ be a binary relation with Properties $2(a)$ and $2(c)$. Then Property $2(b)$ is equivalent to asymmetry of $R$, that is, to $\left(Y, Y^{\prime}\right) \in R \Rightarrow\left(Y^{\prime}, Y\right) \notin R$.

Proof. We first show by contradiction that asymmetry of $R$ follows from Property 2(b). Let $R$ have Property 2(b). Assume that there exist two matrices $Y, Y^{\prime} \in \mathbb{R}^{k \times r}$ with $\left(Y, Y^{\prime}\right) \in R$ and $\left(Y^{\prime}, Y\right) \in R$. We construct an instance with only two (distinct) paths $q, q^{\prime}$ from $s$ to $t$ with $z(q)=Y$ and $z\left(q^{\prime}\right)=Y^{\prime}$. Then $q$ dominates $q^{\prime}$ and vice versa. Hence, $q$ is not robust efficient, but there exists no robust efficient path from $s$ to $t$ dominating $q$. This is a contradiction to Property 2(b). On the other hand, Property 2(b) follows from asymmetry of $R$ due to the finiteness of the set $\mathcal{Q}^{t}$. This has been shown, for example, in [35, Lemma 17] for relations on the solution set, which we can define from the given relation in the objective space.

\section{LABELING FOR THE MULTI-OBJECTIVE ROBUST SHORTEST PATH PROBLEM}

In the following we discuss whether the concepts of robust efficiency presented in Section 2 satisfy the conditions given in Section 3 for using Algorithm 1'. If a concept does not satisfy the conditions, we investigate whether and how the idea of label correcting algorithms can nevertheless be used to find robust efficient solutions.

\section{1 | Multi-scenario efficiency}

Recall that a solution is multi-scenario efficient if it is efficient w.r.t. the deterministic multi-objective edge costs $c(e)=$ $\left(c_{1}\left(e, \xi_{1}\right), \ldots, c_{k}\left(e, \xi_{1}\right), c_{1}\left(e, \xi_{2}\right), \ldots, c_{k}\left(e, \xi_{r}\right)\right)^{T}$. We can hence reduce (MORSP) to a deterministic multi-objective problem and directly use Algorithm 1 to solve it. Note that the set of multi-scenario efficient solutions contains all highly robust efficient solutions as well as the set of all so-called strictly flimsily robust efficient, strictly point-based and strictly set-based minmax robust efficient solutions [7].

\section{2 | Flimsily robust efficiency}

Recall that a solution is flimsily robust efficient if it is efficient for at least one scenario in $\mathcal{U}$. We show that for flimsily robust efficiency, Condition 1 for using Algorithm 1' is satisfied, but not Condition 2. We then extend Algorithm 1' by storing some additional information for each label, such that we can find a complete set of flimsily robust efficient solutions. We also introduce an alternative solution approach which finds a complete set of flimsily robust efficient solutions by applying Algorithm 1' once for each scenario and taking the union of the solution sets.

Lemma 10. Let $q$ be a fimsily robust efficient path for an instance $(G, \mathcal{U}, c, s, t)$ of (MOUSP). Then, for every intermediate node $v$ on $q$, the subpath $q_{s, v}$ is flimsily robust efficient for $(G, \mathcal{U}, c, s, v)$, hence Condition 1 is satisfied.

https://doi.org/10.1002/net.21815 


\section{${ }^{8} \perp$ WILEY}

RAITH ET AL.

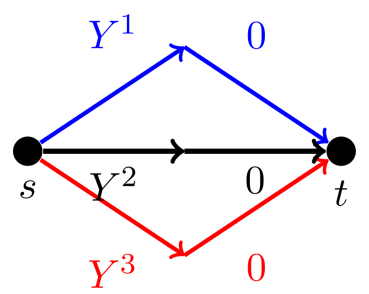

F I G URE 1 Condition 2 is not satisfied for flimsily robust efficiency (Lemma 11) [Color figure can be viewed at wileyonlinelibrary.com]

Proof. Assume that $q_{s, v}$ is not flimsily robust efficient for the instance $(G, \mathcal{U}, c, s, v)$. Then for each $\xi \in \mathcal{U}$ there exists a path $q^{\xi} \in \mathcal{Q}^{v}$ with $z\left(q^{\xi}, \xi\right) \leq z\left(q_{s, v}, \xi\right)$. From

$$
z\left(q^{\xi}, \xi\right) \leq z\left(q_{s, v}, \xi\right) \Rightarrow z\left(q^{\xi}, \xi\right)+z\left(q_{v, t}, \xi\right) \leq z\left(q_{s, v}, \xi\right)+z\left(q_{v, t}, \xi\right)=z(q, \xi)
$$

we conclude that for each $\xi \in \mathcal{U}$ there exists a path from $s$ to $t$ dominating $q$ in scenario $\xi$. This contradicts $q$ being flimsily robust efficient.

The following lemma shows that for flimsily robust efficiency there does not exist a binary relation as required in Condition 2, even for only two objectives and two scenarios.

Lemma 11. For flimsily robust efficiency and $k=r=2$, there does not exist a binary relation with the Property 2(a) given in Condition 2.

Proof. Assume that for $k=r=2$ there exists a binary relation $R \subseteq \mathbb{R}^{k \times r} \times \mathbb{R}^{k \times r}$ with Property 2(a). Consider an instance of (MOUSP) with three disjoint paths as feasible set with the following cost matrices

$$
z\left(\mathcal{Q}^{t}\right)=\left\{Y^{1}:=\left(\begin{array}{ll}
0 & 5 \\
0 & 5
\end{array}\right), Y^{2}:=\left(\begin{array}{ll}
1 & 4 \\
1 & 4
\end{array}\right), Y^{3}:=\left(\begin{array}{ll}
2 & 3 \\
2 & 3
\end{array}\right)\right\}
$$

for example, as in Figure 1. For such an instance, a path $q$ with $z(q)=Y^{2}$ is not flimsily robust efficient, because we have $Y_{(\cdot, 1)}^{1} \leq Y_{(\cdot, 1)}^{2}$ and $Y_{(\cdot, 2)}^{3} \leq Y_{(\cdot, 2)}^{2}$. It follows that $\left(Y^{1}, Y^{2}\right) \in R$ or $\left(Y^{3}, Y^{2}\right) \in R$ because of Property 2(a). However, for instances with $z\left(\mathcal{Q}^{t}\right)=\left\{Y^{1}, Y^{2}\right\}$ resp. $z\left(\mathcal{Q}^{t}\right)=\left\{Y^{3}, Y^{2}\right\}$, a path $q \in \mathcal{Q}^{t}$ with $z(q)=Y^{2}$ is flimsily robust efficient, since $Y_{(\cdot, 2)}^{1} \nless \leq Y_{(\cdot, 2)}^{2}$ and $Y_{(\cdot, 1)}^{3} \nless \leq Y_{(\cdot, 1)}^{2}$ holds. It follows that $\left(Y^{1}, Y^{2}\right) \notin R$ and $\left(Y^{3}, Y^{2}\right) \notin R$, which is a contradiction.

From Lemma 11 it follows that for finding flimsily robust efficient solutions there is no suitable binary dominance relation to be used in Algorithm 1'. It is not sufficient to compare the cost matrices of the paths pairwise without considering additional information in Lines 6-11 of Algorithm 1'. However, if we store the information from previous comparisons, we can eliminate labels representing paths which are not flimsily robust efficient by pairwise comparisons.

Using this idea, we extend Algorithm 1' to Algorithm 2. For each label $l$ we use a binary vector $x(l) \in\{0,1\}^{|\mathcal{U}|}$ to indicate under which scenarios its path has been shown to be dominated. With $q$ being the path represented by $l$ we define $z(l, \xi):=z(q, \xi)$. Algorithm 2 finds a complete set of flimsily robust efficient solutions for instances where each cycle has either cost 0 for each scenario or has cost $\geq 0$ for each scenario.

Note that this condition is stronger than requiring conservativeness w.r.t. $\leq$ for each scenario individually: For example, for $|\mathcal{U}|=\left\{\xi_{1}, \xi_{2}\right\}$ and a cycle $C$ with $z\left(C, \xi_{1}\right)=(0,0)^{T}$ and $z\left(C, \xi_{2}\right)=(1,1)^{T}$ we have $\forall \xi \in \mathcal{U}: z(C, \xi) \geqq 0$, but neither $\forall \xi \in \mathcal{U}: z(C, \xi) \geq 0$ nor $\forall \xi \in \mathcal{U}: z(C, \xi)=0$.

Correctness of this algorithm can be proven similarly to the proof of Theorem 8 , which leads to the following theorem.

Theorem 12. If for each cycle $C$ in $G$ either $\forall \xi \in \mathcal{U}: z(C, \xi)=0$ or $\forall \xi \in \mathcal{U}: 0 \leq z(C, \xi)$, then the output label set of Algorithm 2 represents a complete set of flimsily robust efficient solutions of $(G, \mathcal{U}, c, s, t)$.

https://doi.org/10.1002/net.21815 


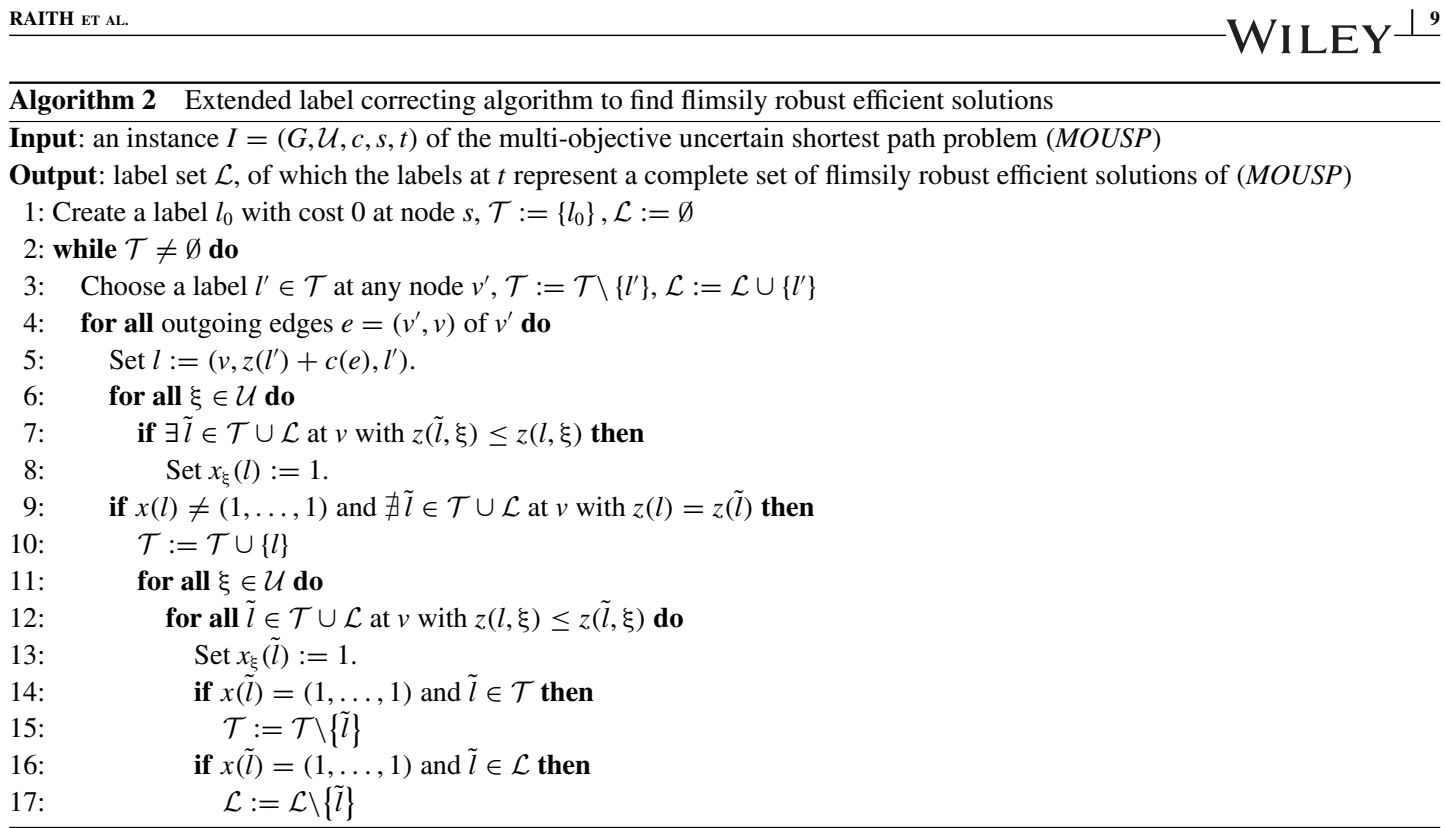

Proof. Note that a label $l$ is deleted if and only if its corresponding vector $x(l)$ contains only ones, that is, if $l$ is dominated in each scenario.

Since each cycle has either cost 0 or has costs $\geq 0$ for each scenario, the cost of a non-simple path is either equal to the cost of the corresponding simple path or dominated by it in each scenario. Analogous to the proof of Theorem 8, whenever the algorithm considers adding the label $l$ of a non-simple path to $\mathcal{T}$, there either exists a label with the same costs or for each scenario $\xi$ there exists a label $l^{\xi}$ dominating $l$. Hence the algorithm stops after finitely many iterations and finds only simple paths.

In Algorithm 2, for every path $p$ from the source a label $l$ with $z(p)=z(l)$ is constructed, if it does not contain a subpath that is dominated in every scenario. From Lemma 10 it follows that no subpath of a flimsily robust efficient path is dominated in every scenario. Analogous to the proof of Theorem 8 , we conclude that for each flimsily robust efficient path $p$ a label $l$ with $z(p)=z(l)$ is found, whereas all labels representing paths which are not flimsily robust efficient are deleted during the algorithm.

An alternative approach to finding a complete set of flimsily robust efficient solutions is presented in Algorithm 3. For each scenario, we use Algorithm 1' to find solutions, which are efficient w.r.t. this scenario. Note that the dominance relation used when applying Algorithm 1' to the subproblems only depends on one scenario. However, when comparing the costs of two labels in Line 6 we only consider them equal if they are equal for each scenario. Therefore, the union of the obtained solution sets is a complete set of flimsily robust efficient solutions. To ensure that Algorithm 3 terminates, we use the same requirement as in Theorem 12: Each cycle $C$ in $G$ has to satisfy either $\forall \xi \in \mathcal{U}: z(C, \xi)=0$ or $\forall \xi \in \mathcal{U}: 0 \leq z(C, \xi)$.

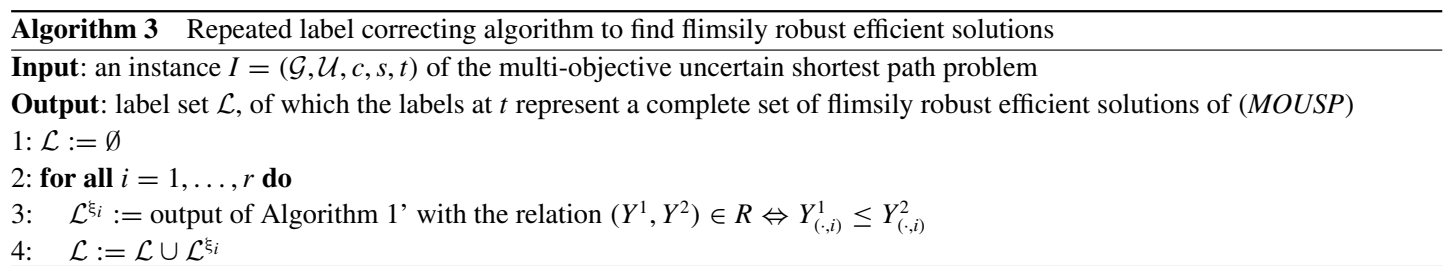

https://doi.org/10.1002/net.21815 
LWILEY— RATTH ET AL

\subsection{Highly robust efficiency}

Recall that a solution is highly robust efficient if it is efficient for each scenario. We show that for highly robust efficiency Condition 1 is satisfied, but that there exists no binary relation with Property 2(b) as required in Condition 2. However, every highly robust efficient solution is flimsily robust efficient as well. We give an algorithm to find a complete set of highly robust efficient solutions which filters the labels obtained by Algorithm 2. Afterwards, we describe an alternative approach in which we apply Algorithm 1' $r$ times and intersect the obtained solution sets.

Lemma 13. Let $q$ be a highly robust efficient path for an instance $(G, \mathcal{U}, c, s, t)$ of (MOUSP). Then for every node $v$ in $q$ the subpath $q_{s, v}$ is highly robust efficient for the instance $(G, \mathcal{U}, c, s, v)$, that is, Condition 1 is satisfied.

Proof. Let $v$ be any node in $q$. Assume that $q_{s, v}$ is not highly robust efficient for $(G, \mathcal{U}, c, s, v)$. Then there exists a path $q^{\prime}$ from $s$ to $v$, which dominates $q_{s, v}$ under at least one scenario $\xi \in \mathcal{U}$. It follows that

$$
z\left(q^{\prime}, \xi\right) \leq z\left(q_{s, v}, \xi\right) \Rightarrow z\left(q^{\prime}, \xi\right)+z\left(q_{v, t}, \xi\right) \leq z\left(q_{s, v}, \xi\right)+z\left(q_{v, t}, \xi\right)=z(q, \xi)
$$

This contradicts $q$ being highly robust efficient.

Lemma 14. For highly robust efficiency, there does not exist a relation with Property 2(b), even for only two objectives and two scenarios.

Proof. Consider the following instance of (MOUSP) for $k=r=2$ with two paths $q_{1}$ and $q_{2}$ with the following cost matrices:

$$
z\left(\mathcal{Q}^{t}\right)=\left\{Y^{1}:=\left(\begin{array}{ll}
0 & 5 \\
0 & 5
\end{array}\right), Y^{2}:=\left(\begin{array}{ll}
1 & 4 \\
1 & 4
\end{array}\right)\right\}
$$

Then, none of the two paths in $\mathcal{Q}^{t}$ is highly robust efficient, because $Y_{(\cdot, 1)}^{1} \leq Y_{(\cdot, 1)}^{2}$ and $Y_{(\cdot, 2)}^{2} \leq Y_{(\cdot, 2)}^{1}$, but both are not dominated by any highly robust efficient path. We conclude that the concept of highly robust efficiency does not have the domination property, hence Property 2(b) cannot hold for any binary relation.

We remark that also Properties 2(a) and 2(c) cannot hold at the same time for the concept of highly robust efficiency.

Without a suitable dominance relation, we cannot use Algorithm 1' to find highly robust efficient solutions. However, since every highly robust efficient solution is also flimsily robust efficient, we can instead compute a complete set of flimsily robust efficient solutions and filter out the highly robust efficient solutions. This can be done efficiently with the help of the additional vectors $x(l)$, which we already introduced for Algorithm 2: At the end of Algorithm 2, a label $l$ is highly robust efficient if $x(l)=(0, \ldots, 0)$. This leads to Algorithm 4 .

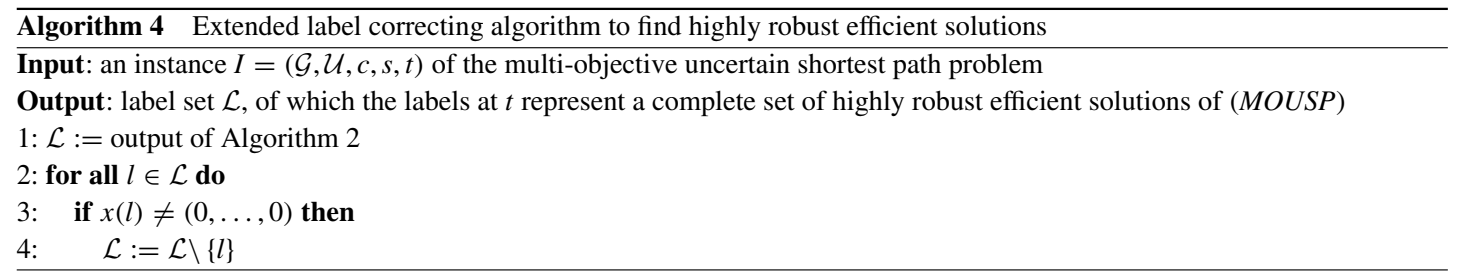

Theorem 15. If for each cycle $C$ in $G$ either $z\left(C, \xi_{i}\right)=0 \forall i=1, \ldots, r$ or $0 \leq z\left(C, \xi_{i}\right) \forall i=1, \ldots, r$, then the output label set $\mathcal{L}$ of Algorithm 4 represents a complete set of highly robust efficient solutions.

Proof. The statement follows directly from Theorem 12 and the fact that every highly robust efficient solution is flimsily robust efficient.

https://doi.org/10.1002/net.21815 
RAITH ET AL.

Similar to Algorithm 3 for finding flimsily robust efficient solutions, an alternative approach for finding highly robust efficient solutions is given in Algorithm 5: For each scenario, we use Algorithm 1' to find efficient solutions w.r.t. this scenario. Then we intersect the obtained solution sets. Here again, when applying Algorithm 1' to the subproblems, the dominance relation only depends on one scenario. However, when comparing the costs of two labels in Line 6 of Algorithm 1', we only consider them equal if they are equal for each scenario, in order to obtain a complete set of highly robust efficient solutions in the end. Hence, Algorithm 5 finds a complete set of highly robust efficient solutions for instances where each cycle $C$ in $G$ satisfies either $\forall \xi \in \mathcal{U}: z(C, \xi)=0$ or $\forall \xi \in \mathcal{U}: 0 \leq z(C, \xi)$.

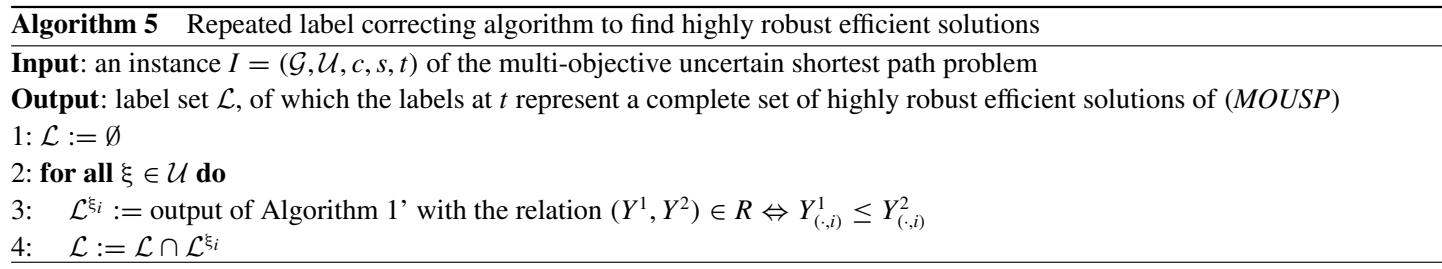

\section{4 | Point-based and set-based minmax robust efficiency}

We show that point-based and set-based minmax robust efficiency both satisfies Condition 2 for using Algorithm 1', but not Condition 1. To be able to nevertheless use a label correcting approach, we propose to use several label sets at each node. This idea was first introduced for single-objective minmax robust shortest path problems in [42].

We first show that both concepts for robust efficiency satisfy Condition 2 by defining a relation for each of the concepts with Properties 2(a)-2(c). Recall that a solution is point-based minmax robust efficient if it is efficient for the deterministic multi-objective problem

$$
\left(S P_{\max }\right) \quad \min _{q \in Q} \bar{z}(q):=\left(\begin{array}{c}
\max _{\xi \in \mathcal{U}} z_{1}(q, \xi) \\
\vdots \\
\max _{\xi \in \mathcal{U}} z_{k}(q, \xi)
\end{array}\right) .
$$

$\left(S P_{\max }\right)$ is not a classical multi-objective robust shortest path problem, because suitable edge costs are not known in advance. Therefore, it cannot simply be solved with a deterministic multi-objective labeling algorithm. However, by identifying $z(q)$ with $\bar{z}(q)$, the $\leq$-relation on $\mathbb{R}^{k}$ induces a binary relation $R^{\text {point }} \subseteq \mathbb{R}^{k \times r} \times \mathbb{R}^{k \times r}$, which is defined as

$$
\left(Y, Y^{\prime}\right) \in R^{\text {point }} \Leftrightarrow\left(\begin{array}{c}
\max _{i=1, \ldots, r} Y_{1, i} \\
\vdots \\
\max _{i=1, \ldots, r} Y_{k, i}
\end{array}\right) \leq\left(\begin{array}{c}
\max _{i=1, \ldots, r} Y_{1, i}^{\prime} \\
\vdots \\
\max _{i=1, \ldots, r} Y_{k, i}^{\prime}
\end{array}\right) .
$$

It is easy to check that this relation has the properties required in Condition 2. Now, we consider set-based minmax robust efficient solutions: A path $q \in \mathcal{Q}^{t}$ is set-based minmax robust efficient if there is no solution $q^{\prime} \in \mathcal{Q}^{t}$ with $z_{\mathcal{U}}\left(q^{\prime}\right) \subseteq z_{\mathcal{U}}(q)-\mathbb{R}_{\geq}^{k}$. This definition directly leads to a suitable binary relation on $\mathbb{R}^{k \times r}$ : Given $k, r \in \mathbb{N}$ we construct the binary relation $R^{\text {set }} \subseteq \mathbb{R}^{k \times r} \times \mathbb{R}^{k \times r}$ :

$$
\left(Y, Y^{\prime}\right) \in R^{\mathrm{set}} \Leftrightarrow \bigcup_{i=1, \ldots, r}\left\{Y_{(\cdot, i)}\right\} \subseteq \bigcup_{i=1, \ldots, r}\left\{Y_{(\cdot, i)}^{\prime}\right\}-\mathbb{R}_{\geq}^{k}
$$

Again, it can be checked that this relation fulfills Condition 2.

For $k=1$, both point-based and set-based minmax robust efficiency reduce to the single-objective concept of minmax robustness. The single-objective minmax robust shortest path problem is already NP-hard [32,42]. Efficient labeling and dynamic programming algorithms cannot be used, because Bellman's principle of optimality is not satisfied.

https://doi.org/10.1002/net.21815 
In [42] a pseudo-polynomial algorithm for the single-objective minmax robust shortest path problem with positive integer edge lengths is given. Instead of a single label at each node $v$, for each possible cost of the part of the path that has not been looked at yet, a label is saved at $v$.

In order to find a complete set of [set-based/point-based] minmax robust efficient solutions, we transfer this idea to our label correcting algorithm by adding a prediction matrix as a fourth component to each label: A label $l=\left(v, z(l), l^{\prime}, A\right)$ now consists of a node $v$, a cost matrix $z(l)$, a predecessor label $l^{\prime}$ as before, and a prediction matrix $A \in \mathbb{Z}^{k \times r}$. We also define a function $a$ with $a(l):=A$, assigning the prediction matrix to label $l$. A path from $s$ to $v$ can be represented by several labels with different prediction matrices. The prediction matrix contains the assumed costs for continuing the path from $v$ to $t$.

In the beginning of the algorithm, component-wise upper and lower bounds $A_{i, j}^{\min }$ and $A_{i, j}^{\max }$ for the cost of a simple path in $G$ are computed. For example, one obtains suitable bounds by

$$
A_{i, j}^{\min }:=\sum_{e \in E} \min \left\{0, c_{i}\left(e, \xi_{j}\right)\right\}, \quad A_{i, j}^{\max }:=\sum_{e \in E} \max \left\{0, c_{i}\left(e, \xi_{j}\right)\right\} .
$$

With $A \leqq A^{\prime}$ we denote that matrix $A$ is component-wise smaller or equal to matrix $A^{\prime}$.

Algorithm 6 is correct for instances with integer edge costs which are conservative w.r.t. $\left[R^{\text {point }} / R^{\text {set }}\right]$. However, it can easily be adjusted to rational edge costs by allowing $A \in \mathbb{Q}^{k \times r}$ and adjusting the step length by which $A_{i, j}$ is increased in Lines 3 to 6 .

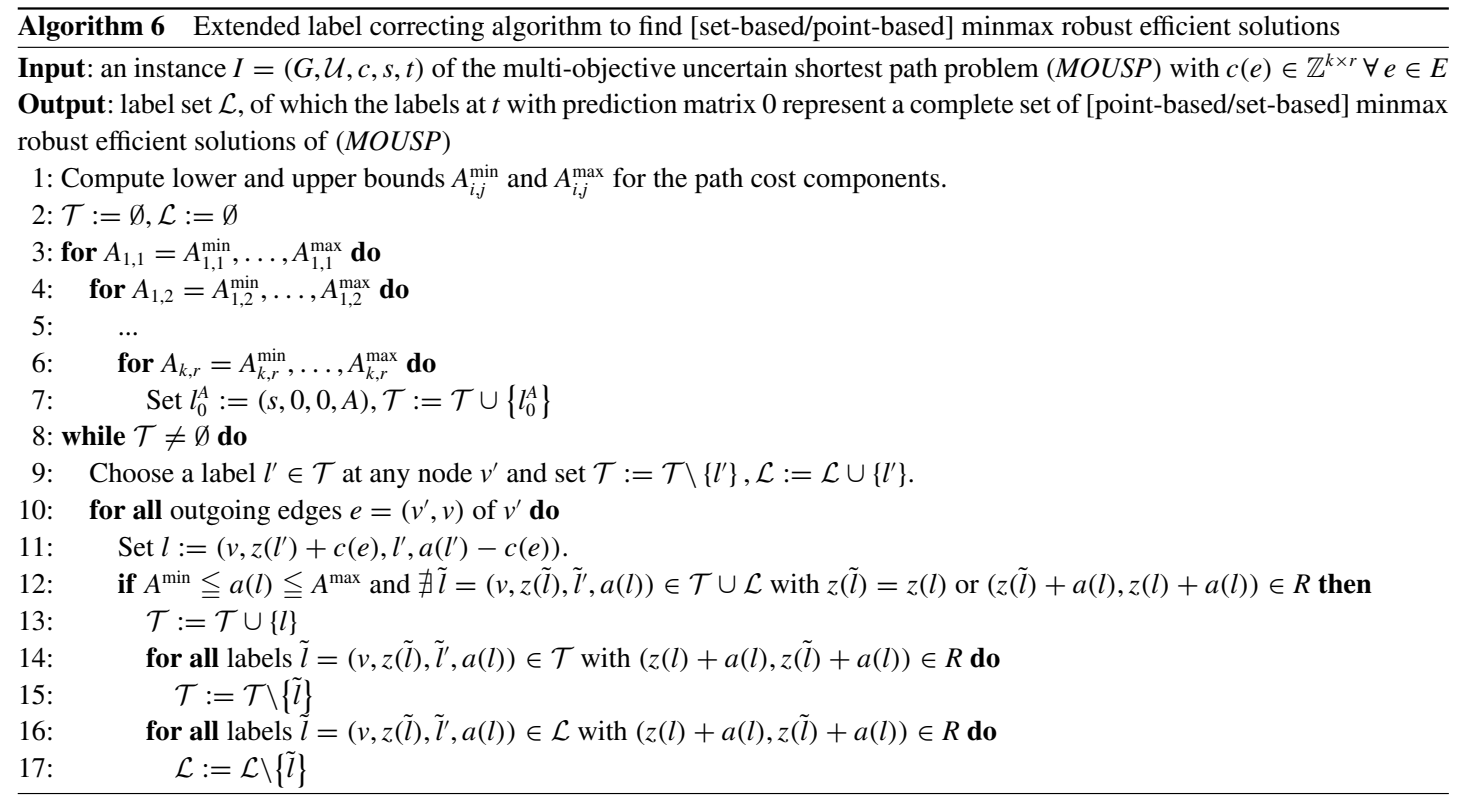

Theorem 16. Let $R$ be the relation $\left[R^{\text {point }} / R^{\text {set }}\right]$. Let the instance $I=(G, \mathcal{U}, c, s, t)$ be conservative w.r.t. $R$ and $c(e) \in \mathbb{Z}^{k \times r} \forall e \in E$. Then the output of Algorithm 6 is a complete set of [point-based/set-based] minmax robust efficient solutions.

Proof. We first show that Algorithm 6 stops after finitely many iterations. We then show that $q \in \mathcal{Q}^{t}$ is [pointbased/set-based] minmax robust efficient $\Leftrightarrow$ at the end of Algorithm 6, there is a label $l \in \mathcal{L}$ at node $t$ with cost $z(l)=z(q)$ and prediction matrix $a(l)=0$.

First note that in contrast to Algorithm 1 and Algorithm 2, in Algorithm 6 labels corresponding to non-simple paths are not immediately sorted out in Line 12, since Line 12 only compares labels having the same prediction matrix. However, since there are only $\hat{m}:=\prod_{i=1, \ldots, k, j=1, \ldots, r}\left(A_{i, j}^{\max }-A_{i, j}^{\min }+1\right)$ different prediction matrices, no path

https://doi.org/10.1002/net.21815 
for which a label is added to $\mathcal{L}$ contains a node $v$ more than $\hat{m}$ times: A path $p$ containing $v$ more than $\hat{m}$ times has at least $\hat{m}+1$ subpaths ending in $v$ (including $p$ itself). Hence, at least two of the corresponding labels have the same prediction matrix. However, as soon as a label $l^{\prime}$ in $v$ is created with the same prediction matrix as a predecessor label $l$ in $v$, we have

$$
z(l)=z\left(l^{\prime}\right) \text { or }\left(\mathrm{z}(\mathrm{l})+\mathrm{a}(\mathrm{l}), \mathrm{z}\left(\mathrm{l}^{\prime}\right)+\mathrm{a}\left(\mathrm{l}^{\prime}\right)\right) \in \mathrm{R}
$$

since the instance is conservative, and $l^{\prime}$ is discarded in Line 12. We now show that $q \in \mathcal{Q}^{t}$ is [point-based/setbased] minmax robust efficient $\Leftrightarrow$ at the end of the algorithm, there is a label $l \in \mathcal{L}$ at node $t$ with $\operatorname{cost} z(l)=z(q)$ and prediction matrix $a(l)=0$.

$\Rightarrow$ : Let $q$ be a [point-based/set-based] minmax robust efficient solution. Without loss of generality we can assume that $q$ is a simple path: Because the instance is conservative w.r.t. $R$, for any non-simple path $q$ there either exists an equivalent simple path or $q$ is not [point-based/set-based] minmax robust efficient.

Let $l$ be the first label at $t$ added to $\mathcal{T}$ with cost $z(l)=z(q)$ and $a(l)=0$. Then $l \in \mathcal{L}$ at the end of the algorithm, because there exists no $q^{\prime} \in \mathcal{Q}^{t}$ with $\left(z\left(q^{\prime}\right), z(q)\right) \in R$. It remains to show that a label with cost $z(l)=z(q)$ and $a(l)=0$ is added to $\mathcal{T}$. We show by induction that for each node $v$ on $q$, a label with cost $z\left(q_{s, v}\right)$ and prediction matrix $z\left(q_{v, t}\right)$ is added to $\mathcal{T}$ during the algorithm.

In Line 7, a label at node $s$ with length 0 and prediction matrix $z(q)$ is added to $\mathcal{T}$, since $A^{\text {min }} \leqq z(q) \leqq A^{\max }$. Let $\left(v^{\prime}, v\right)$ be an edge in $q$. Assume that a label $l^{\prime}$ at $v^{\prime}$ with $z\left(l^{\prime}\right)=z\left(q_{s, v^{\prime}}\right)$ and $a\left(l^{\prime}\right)=z\left(q_{v^{\prime}, t}\right)$ is added to $\mathcal{T}$ during the algorithm. Since $q$ is [point-based/set-based] minmax robust efficient, there is no path $q^{\prime}$ with $\left(z\left(q^{\prime}\right)+z\left(q_{v^{\prime}, t}\right), z\left(q_{s, v^{\prime}}\right)+z\left(q_{v^{\prime}, t}\right)\right) \in R$. Hence, $l^{\prime}$ is removed from $\mathcal{T}$ and added to $\mathcal{L}$ in some iteration of Line 9 . Then, in Line 11 a label $l$ with $z(l)=z\left(q_{s, v^{\prime}}\right)+c\left(v^{\prime}, v\right)=z\left(q_{s, v}\right)$ and $a(l)=z\left(q_{v^{\prime}, t}\right)-c\left(v^{\prime}, v\right)=z\left(q_{v, t}\right)$ is created. Since $q$ is [point-based/set-based] minmax robust efficient, there is no path $q^{\prime}$ with $\left(z\left(q^{\prime}\right)+z\left(q_{v^{\prime}, t}\right), z\left(q_{s, v^{\prime}}\right)+\right.$ $\left.z\left(q_{v^{\prime}, t}\right)\right) \in R$ and $l$ is added to $\mathcal{T}$, unless there already is a label in $\mathcal{T} \cup \mathcal{L}$ with the same cost and prediction matrix.

We conclude that for each node $v$ on $q$, a label with cost $z\left(q_{s, v}\right)$ and prediction matrix $z\left(q_{v, t}\right)$ is added to $\mathcal{T}$ during the algorithm, in particular for $v=t$.

$\Leftarrow$ : The dominance checks in Lines $12-17$ guarantee that for any two labels $l, l^{\prime}$ in $\mathcal{L}$ we have

$$
\left(z(l)+a(l), z\left(l^{\prime}\right)+a\left(l^{\prime}\right)\right) \notin R,
$$

thus in particular for our output labels (with $a(l)=a\left(l^{\prime}\right)=0$ )

$$
\left(z(l), z\left(l^{\prime}\right)\right) \notin R
$$

no two paths in the output dominate each other.

Note that this algorithm also only returns labels representing simple paths: If a non-simple path $p$ is not dominated, the cost of all its cycles is 0 and the label representing the respective simple path with prediction matrix 0 was constructed earlier than the label representing $p$.

\section{5 | Summary}

Table 1 summarizes which properties of the two conditions given in Section 3 are satisfied for each of the considered concepts of robust efficiency and which algorithms can be used to find a complete set of robust efficient solutions. All presented algorithms are pseudo-polynomial for a fixed number of objectives and scenarios and integer edge costs: Carefully counting the steps shows that for polynomially bounded integer edge costs the algorithms run in polynomial time. This cannot be expected if the number of scenarios is unbounded, since the single-objective minmax robust shortest path problem with integer edge costs is then already strongly NP-hard [42].

https://doi.org/10.1002/net.21815 
${ }^{14} \perp$ WILEY- RATTH Fr AL.

TABLE 1 Summary of which conditions are satisfied for which concept of robust efficiency and which algorithms can be used to solve (MORSP)

\begin{tabular}{|llll|}
\hline Concept of robust efficiency & Condition 1 & Condition 2 & Algorithms \\
\hline Multi-scenario efficiency & Yes & Yes & 1 \\
\hline Flimsily robust efficiency & Yes & No & 2,3 \\
\hline Highly robust efficiency & Yes & No & 4,5 \\
\hline Point-based minmax robust efficiency & No & Yes & 6 \\
\hline Set-based minmax robust efficiency & No & Yes & 6 \\
\hline
\end{tabular}

\section{5 | EXPERIMENTS}

In the previous section we developed several algorithms for finding robust efficient solutions. These can be classified into two groups:

- Extended labeling algorithms: Algorithms that use an extension of Algorithm 1' based on the Conditions 1 and 2 we introduced in Section 3. These are Algorithms 2, 4, and 6 for flimsily, highly, and point-based/set-based minmax robust efficiency.

- Repeated labeling algorithms: Algorithms that rely on repeated application, for every scenario, of Algorithm 1'. These are Algorithms 3 and 5 for flimsily and highly robust efficiency

The main goal of this section is to compare these two classes of algorithms. Since we have algorithms from both classes for the two concepts of flimsily and highly robust efficient solutions we take these as basis for our experiments, that is, the following four algorithms presented in this article are tested and compared in detail:

- EL-Flimsily is Algorithm 2, the extended label correcting algorithm to find flimsily robust efficient paths.

- RL-Flimsily is Algorithm 3, where Algorithm 1' is applied $r$ times to find flimsily robust efficient paths.

- EL-Highly is Algorithm 4, which applies Algorithm 2 (EL-Flimsily) and identifies highly robust efficient solutions from the output.

- RL-Highly is Algorithm 5, where Algorithm 1' is applied $r$ times to find highly robust efficient paths.

In addition, we also present some results showing particularities of the extended labeling algorithms for finding point-based and set-based minmax robust efficient solutions:

- EL-PB is Algorithm 6 with dominance relation $R^{\text {point }}$, the extended label correcting algorithm to find point-based minmax robust efficient paths.

- EL-SB is Algorithm 6 with dominance relation $R^{\text {set }}$, the extended label correcting algorithm to find set-based minmax robust efficient paths.

Since our test instances have positive edge lengths, we set the lower bounds $A_{i, j}^{\min }$ needed for EL-PB and EL-SB to 0. Further, we calculate the upper bounds $A_{i, j}^{\max }$ as the sum of the $|V|-1$ largest costs for each objective $i$ and scenario $j$.

All algorithms were implemented in $\mathrm{C}++$, compiled with gcc version 5.4.0, and run under Ubuntu 16.04.2 on a laptop with $3 \mathrm{GHz}$ processor and 16GB RAM. Results are analyzed and plots and tables are generated in the statistical computing environment R [36].

\section{1 | Test instances}

We test the presented algorithms based on two types of network instances, grid networks and so-called NetMaker networks.

\subsection{1 | Grid networks}

Grid networks are introduced in $[11,37]$, where nodes are arranged in a rectangular grid of height $h$ and width $w$. The start node $s$ and end node $t$ are outside the grid, namely on the left and right, with edges connecting them to all left-most and right-most

https://doi.org/10.1002/net.21815 


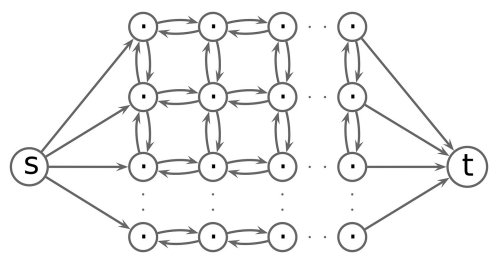

F I G U RE 2 Structure of grid networks

nodes, respectively, as shown in Figure 2. The (integer) edge cost components are randomly chosen from a discrete uniform distribution between 1 and a given upper bound $c$. We construct one set of random grid network instances where the costs for all scenarios are chosen randomly. For the other set of correlated grid network instances the cost vector of scenario $\xi_{1}$ is randomly generated, and the other cost vectors $c(e, \xi)$ are generated based on $c\left(e, \xi_{1}\right)$, where costs are now randomly generated such that $c(e, \xi) \in\left\{\max \left\{1, c\left(e, \xi_{1}\right)-3\right\}, \ldots, c\left(e, \xi_{1}\right)+3\right\}$.

\subsection{2 | NetMaker networks}

So-called NetMaker networks were first introduced by [40] for testing a bi-objective shortest path algorithm, and also used by others [37]. A random Hamiltonian cycle ensures the network is connected. Other edges $\left(v, v^{\prime}\right)$ are randomly generated for each node $v$. A random number of edges with tail node $v$ are generated where the number of such edges lies in the interval $\left\{e_{\min }, e_{\min }+1, \ldots, e_{\max }\right\}$ with equal probability. NetMaker also limits how far these edges can reach: Assuming all nodes are numbered $\{1,2,3, \ldots,|V|\}$, with 1 being the start node and $|V|$ the target node, any edge $\left(v, v^{\prime}\right)$ with tail node $v$ can only reach a node $v^{\prime} \in\left\{v-\left\lceil\frac{I}{2}\right\rceil, \ldots, v-1, v, v+1, \ldots, v+\left\lceil\frac{I}{2}\right\rceil\right\}$. This prevents paths from $s$ to $|V|$ with very few edges, which then would easily dominate all other paths. In the following $I=10$ is chosen.

In the original bi-objective instances $[37,40]$, for each edge it is first randomly determined which interval edge costs fall into:

$$
\begin{aligned}
& \text { 1. } c_{1}(e) \in\{1,2, \ldots, 33\} \text { and } c_{2}(e) \in\{67,68, \ldots, 100\} \text {, or } \\
& \text { 2. } c_{1}(e) \in\{67,68, \ldots, 100\} \text { and } c_{2}(e) \in\{1,2, \ldots, 33\} \text {. }
\end{aligned}
$$

The actual edge cost is then randomly chosen from the respective set, with uniform distribution. To generate instances with $k=3$, for each edge, we randomly allocate exactly one of the three cost intervals $\{1,2, \ldots, 33\},\{34,35, \ldots, 66\}$, or $\{67,68, \ldots, 100\}$ to each edge cost component, and randomly select the actual cost value from the respective interval.

We generate NetMaker network instances with random scenarios. For any edge $e$ and cost component $k$, all scenarios' costs $c_{k}(e, \xi)$ will be randomly chosen from the same interval associated with $k$ and $e$. Correlated NetMaker instances are constructed as for grid networks by randomly generating the cost vector $c\left(e, \xi_{1}\right)$ according to scenario $\xi_{1}$, and generating the others such that $c(e, \xi) \in\left\{\max \left\{1, c\left(e, \xi_{1}\right)-3\right\}, \ldots, c\left(e, \xi_{1}\right)+3\right\}$. The costs of edges, for all cost components and scenarios, on the Hamiltonian cycle are chosen like all other edge costs, and multiplied by a factor of 10 to penalize their use. In this aspect our instance generation may differ from [40].

\section{2 | Finding flimsily and highly robust efficient solutions}

This section analyses solution numbers, difficulty of problem instances and runtimes of the different algorithms introduced for finding flimsily and highly robust efficient solutions.

\subsection{1 | Computational setup}

We consider instances based on grid networks with two or three objectives $(k=2,3)$ with each combination of the following parameters:

- grid height $h=10,20,30,40$,

- grid width $w=10,20,30,40$,

https://doi.org/10.1002/net.21815 
${ }^{16} \perp$ WILEY- RAITH Fr AL.

- number of scenarios $r=2,4,6,8$, and

- costs chosen from $\{1,2, \ldots, c\}$ with $c=10,100$.

The edge cost range used in $[11,37],\{1, \ldots, 10\}$, is therefore considered in our setting. We furthermore test a smaller range of instances with four objectives $(k=4)$ and the following parameters:

- grid height $h=10,20,30$,

- grid width $w=10,20,30$,

- number of scenarios $r=2,4$, and

- costs chosen from $\{1,2, \ldots, c\}$ with $c=10,100$.

We consider instances based on NetMaker networks with each combination of the following parameters:

- number of objectives $k=2,3$

- number of nodes $n=101,201,401,801,1201$, also $n=1601$ only for $k=2$

- number of outgoing edges for each node in $\{1,2, \ldots, 7\}$, that is, $e_{\min }=1, e_{\max }=7$.

- This ensures that, on average, there are 4-5 outgoing edges for each node. This leads to similar network density in grid and NetMaker networks.

- number of scenarios $r=2,4,6,8$.

Tables A1-A16 in the appendix list $|V|,|E|$ and the choice of parameters $h, w, r, c$ for each grid instance; similarly NetMaker instance parameters are listed in Tables A17-A24. Runtime (in seconds) is recorded for each algorithm in the tables. When runtime exceeds 1 hour, runs were not completed and the runtime is shown in the tables as $>3600.00$. The tables also list the number of solutions found for each instance, where the column "sols" refers to the number of obtained flimsily and highly robust efficient solutions, respectively.

For most experiments a single instance was generated for each set of parameters. Since costs in grid networks, as well as edges and costs in NetMaker networks, are randomly chosen, instances for the same set of parameters can vary. For NetMaker instances we analyze the results over repeated runs (20 for $k=2$ and 10 for $k=3$ ) for each set of problem parameters. Hence, minimum, maximum and averages are reported in Tables A17-A24, and plots in the following subsections show average results and error bars (one standard deviation), where applicable. For grid networks, where only the edge costs, not the network structure itself, are variable, we investigate the variability of runtimes and numbers of solutions for $k=2$ objectives on 20 instances for each parameter set (see Section 5.2.2). Results for $k=2$ in Tables A1-A4 and A9-A12 also report minimum, maximum and average, and plots are based on average results, with error bars where applicable.

\subsection{2 | Comparison of extended and repeated labeling algorithms}

Tables A1-A24 show that the runtimes of the extended labeling algorithms EL-Flimsily and EL-Highly are in general similar for each instance, which is expected as they both apply Algorithm 2. Similarly, runtimes of the repeated labeling algorithms RL-Flimsily and RL-Highly are similar as they also both apply Algorithm 1' $r$ times. When runtimes differ this is due to the complexity of the filtering process to identify all flimsily or highly robust efficient solutions. Hence, we will illustrate all results about runtimes only for flimsily robust efficiency. The same trends can be observed for highly robust efficiency as well, if not stated otherwise.

Figures 3 and 4 show runtimes of both classes of algorithms for finding flimsily robust efficient solutions in grid networks with correlated and random edge costs. The horizontal axis shows network height and width of the instances, and the two different types of algorithms are shown as circles and triangles with points slightly offset to make them easier to compare. The white background color indicates results for $c=10$, and gray background for $c=100$. For $k=2$ average runtimes are shown by the marker with error bars indicating one standard deviation. Furthermore, the number of scenarios in an instance is color-coded.

We observe that it is faster to solve Algorithm 1' $r$ times, as in the repeated labeling algorithms RL-Flimsily and RL-Highly, than to tackle the full problem with the extended labeling algorithms EL-Flimsily and EL-Highly, respectively. This is due to the increased complexity of the algorithms as the additional vector $x$ has to be maintained to correctly determine dominance of flimsily robust efficient labels. Discarding a label because it is dominated may only be possible later during the algorithm as a

https://doi.org/10.1002/net.21815 
RAITH ET AL.

WI LEY $\stackrel{17}{\perp 1}$

Finding flimsily robust efficient solutions, correlated instances, $\mathrm{k}=2$

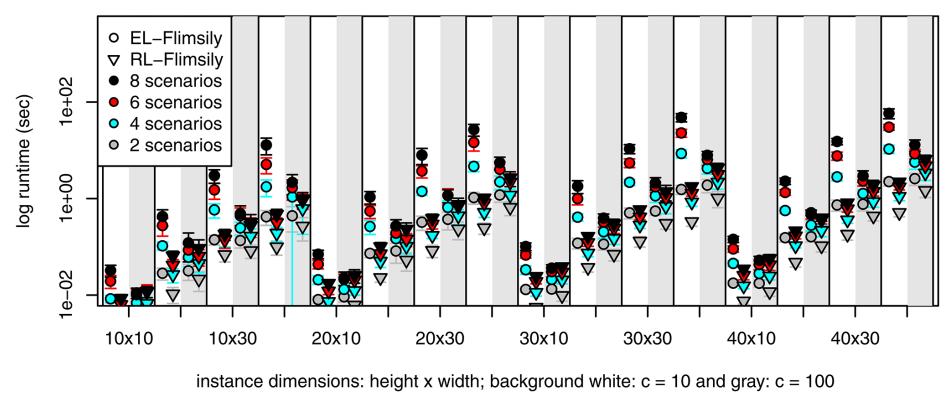

Finding flimsily robust efficient solutions, correlated instances, $\mathrm{k}=3$

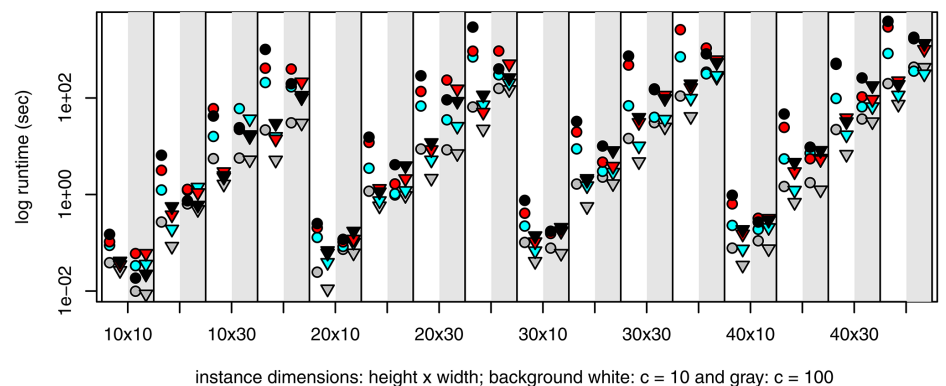

Finding flimsily robust efficient solutions, correlated instances, $k=4$

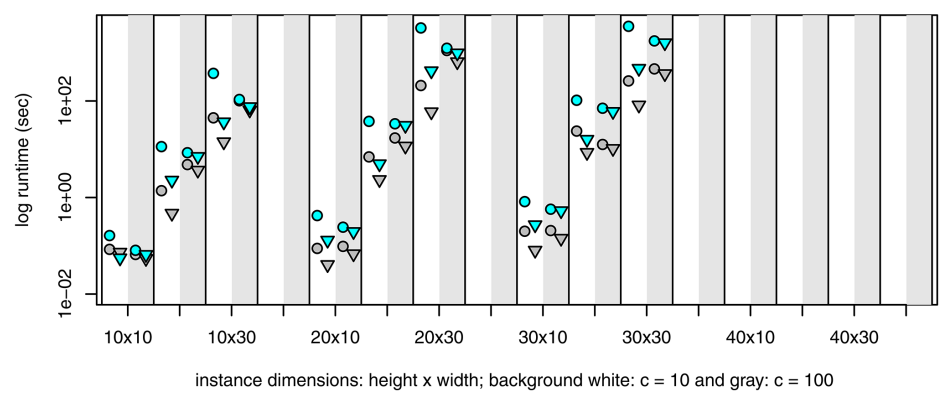

F I G U R E 3 Runtimes for finding flimsily robust efficient solutions of correlated grid instances with $k=2$ (top), $k=3$ (center), $k=4$ (bottom), only showing runtimes exceeding 0.01 seconds. For $k=2$ averages are shown with error bars indicating one standard deviation [Color figure can be viewed at wileyonlinelibrary.com]

label can only be discarded once it is dominated in all scenarios, when $x=(1,1, \ldots, 1)$. Hence, before it is confirmed that a label cannot be flimsily robust efficient, it may have been extended to many other nodes. The advantage of solving Algorithm 1' $r$ times, as in RL-Flimsily and RL-Highly, is that the subproblems have fewer labels at the nodes as dominance can be established earlier, namely as soon as a label is dominated in the current scenario. This means that labels are less often unnecessarily carried forward by the algorithms.

For random instances (Figure 3) runtimes of RL-Flimsily and EL-Flimsily increase, when the maximum cost increases from $c=10$ to $c=100$. For correlated instances, runtimes of RL-Flimsily increase, whereas runtimes of EL-Flimsily decrease when the maximum cost increases. The number of flimsily robust efficient solutions tends to decrease (see corresponding tables), and the runtime of EL-Flimsily benefits from this. Finally, the repeated runs for the same set of instance parameters with $k=2$ show

https://doi.org/10.1002/net.21815 


\section{${ }^{18}$ WILEY}
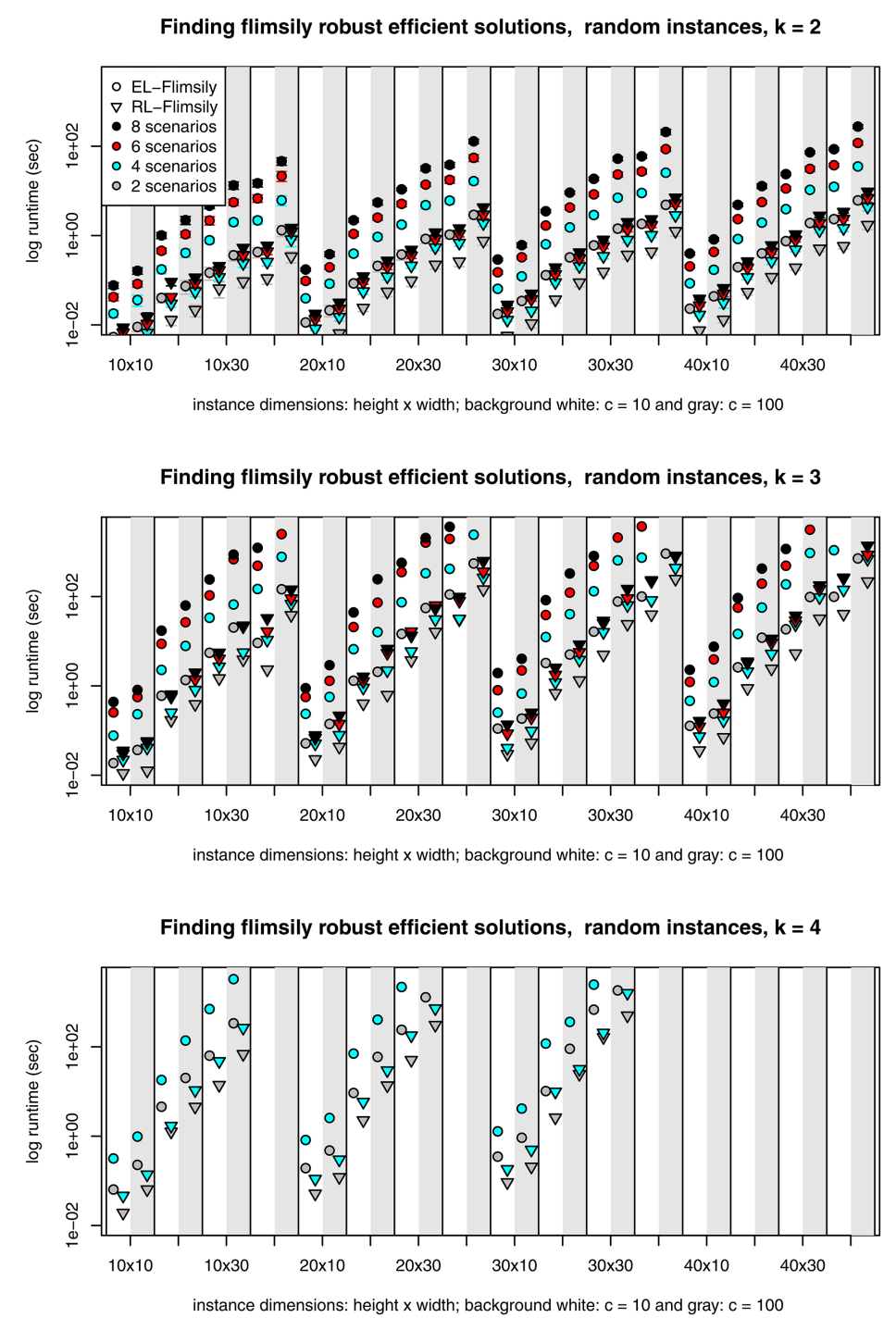

F I G URE 4 Runtimes for finding flimsily robust efficient solutions of random grid instances with $k=2$ (top), $k=3$ (center), $k=4$ (bottom), only showing runtimes exceeding 0.01 seconds. For $k=2$ averages are shown with error bars indicating one standard deviation [Color figure can be viewed at wileyonlinelibrary.com]

that instances are of varying difficulty in terms of number of solutions and runtime, as expected. For instances with random scenarios the effect was minor; that is runtimes for one set of parameters generally do not overlap with those for a different set of parameters. While runtimes for similar sets of instance parameters can overlap for correlated instances, for example, for 6 and 8 scenarios, this does not tend to occur for parameter values that differ more, for example, 2 and 8 scenarios. Therefore we conclude that general trends observed for grid networks in this section are valid even though experiments were only run for one instance per set of parameters when $k=3,4$.

Figure 5 shows runtimes for NetMaker instances. Here, the average runtimes of the two classes of algorithms, indicated by circles and triangles, are shown for networks of different sizes. The error bars indicate one standard deviation. The number of

https://doi.org/10.1002/net.21815 
RAITH ET AL.

WILEY ${ }^{\perp 19}$

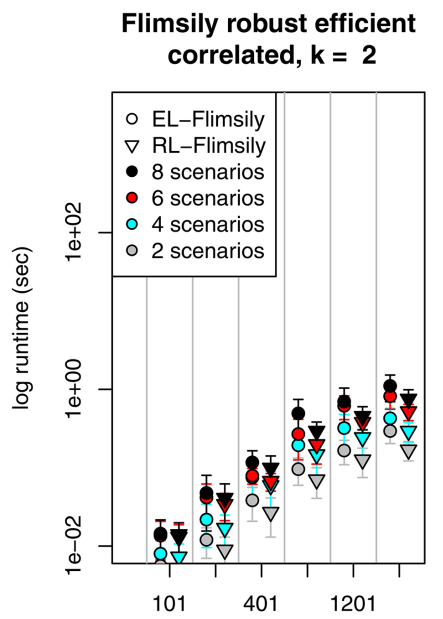

instance dimensions: nodes

Flimsily robust efficient random, $k=2$

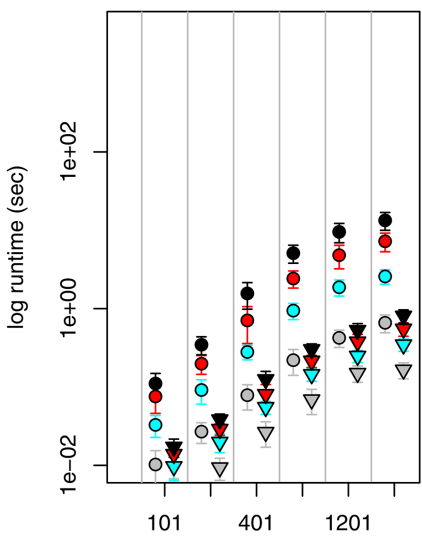

instance dimensions: nodes

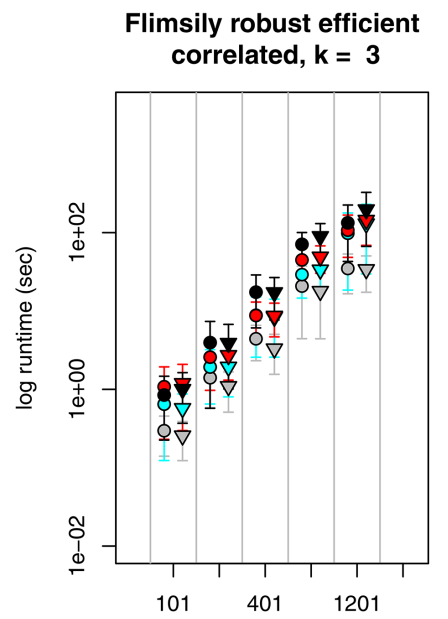

instance dimensions: nodes

Flimsily robust efficient random, $k=3$

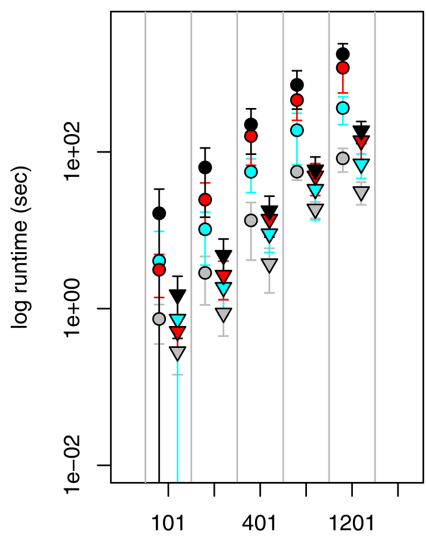

instance dimensions: nodes

F I G U RE 5 Runtimes for finding flimsily robust efficient solutions of NetMaker instances with $k=2$ (left), $k=3$ (right), correlated (top) and random (bottom) scenarios, only showing runtimes exceeding 0.01 seconds. Markers indicate average runtimes, and error bars one standard deviation [Color figure can be viewed at wileyonlinelibrary.com]

scenarios is color-coded and the subfigures show instances with $k=2$ or $k=3$ objectives. We observe that for this network type, the extended algorithm is sometimes faster than the repeated algorithm, in particular for correlated scenarios and $k=3$ (Figure 5). This is illustrated in Figure 6, where runtimes of the EL-Flimsily and RL-Flimsily algorithms are plotted for the same set of parameters, and the straight line indicates where runtimes would be equal. It again confirms that for some correlated instances with $k=3$ EL-Flimsily is faster than RL-Flimsily.

This can be explained by the fact that for correlated scenarios a path is more likely to dominate another in every scenario than for random scenarios. As explained above, in EL-Flimsily, a label that does not represent a flimsily robust efficient path may produce many successor labels until a dominating label is found for each scenario. In instances with correlated scenarios, however, a label is often dominated for all scenarios, as soon as it is dominated for one scenario, hence one dominating label

https://doi.org/10.1002/net.21815 


\section{${ }^{20} \perp$ WILEY}

RAITH et AL.

(a) Flimsily robust efficient, correlated instances

(b) Flimsily robust efficient, random instances
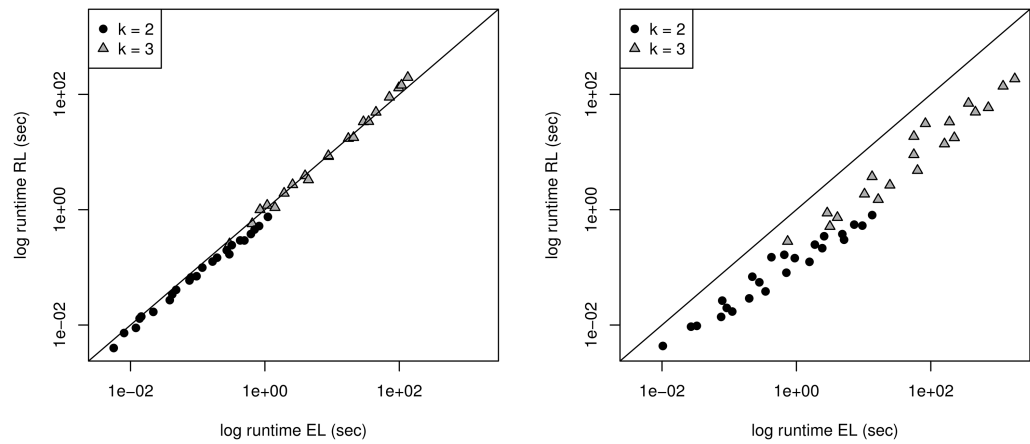

F I G U R E 6 Comparing the average runtimes for NetMaker instances for finding flimsily robust efficient solutions for (a) correlated and (b) random instances. The straight line indicates where instances would have equal runtimes for both algorithms

suffices to discard it. The runtime of EL-Flimsily benefits from this, whereas RL-Flimsily needs to repeat the whole labeling procedure $r$ times, even if the costs are identical for all scenarios. This effect can also be observed for grid networks, when comparing the runtime of random and correlated instances in Figures 3 and 4 (in particular for $c=100$ ); however, RL-Flimsily is still faster even for correlated grid instances. The difference between runtimes for random and correlated instances is discussed in more detail in Section 5.2.4.

\subsubsection{Runtime with respect to network size and number of scenarios and objectives for both classes}

For grid networks, Figures 3 and 4 show how instances become more challenging as the height or width of the problem instance increases. This increase is more significant for increasing width than for increasing height, which is explained in Section 5.2.5.

Comparing the plots for $k=2,3,4$, which all use the same scale for runtime, it is apparent that increasing $k$ significantly increases the runtime. Further, for higher numbers of objectives, the parameters $h$ and $w$ influence runtime more, as can be seen by comparing the difference between runtimes for different network sizes in each of the plots.

In addition, the number of scenarios is color-coded in the figures and illustrates that the runtime of both classes of algorithms mostly increases as the number of scenarios increases. However, this trend is not as clear as for increasing size of networks and number of objectives, as can, for example, be observed for several instances with 6 or 8 scenarios, in particular for correlated instances.

Similarly, Figure 5 shows that also for NetMaker instances increasing the numbers of nodes, objectives and scenarios generally lead to increasing runtimes.

\subsection{4 | Differences between correlated and random scenarios}

We also analyze differences in runtime and number of solutions for random and correlated instances with the same parameters.

For grid instances, by comparing Figures 3 and 4 (and corresponding tables), one can observe that runtimes for EL-Flimsily tend to be lower for correlated instances than for random instances, in particular for $c=100$. An explanation for this is given in Section 5.2.2. In Figure 7 we analyze differences in runtime of RL-Flimsily and number of solutions found for random and correlated scenarios. Every point in Figure 7a represents the number of solutions of a grid instance with parameters $k, r, h, w$ with correlated scenarios (horizontal axis) and random scenarios (vertical axis). For $k=2$ it shows the average number of solutions of all 20 instances with the same set of parameters. It should be noted that, for all instances contributing to the same point in the figure, the instance parameters $k, r, h, w$ are identical, but instances have different randomly generated costs associated with the edges. The straight line indicates where the number of solutions for random and correlated instances is identical. The figures distinguish instances with $c=10$ (circles) and $c=100$ (triangles).

Figure 7a shows that the number of flimsily robust efficient solutions found for instances with correlated and random scenarios is often similar but some random instances with $c=10$ tend to have more solutions than their correlated counterpart (there are more points further above the line than below). For $c=100$ a clear trend for more solutions in random scenarios can be seen. Runtimes (or average runtimes, for $k=2$ ) of RL-Flimsily in Figure $7 \mathrm{~b}$ tend to be similar for correlated and random

https://doi.org/10.1002/net.21815 
(a) No of flimsily robust eff. solutions (grid)

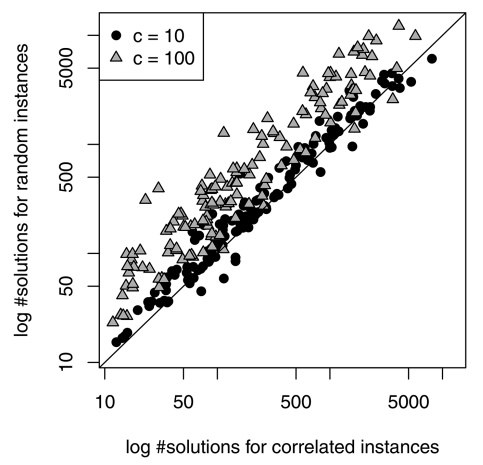

(c) No of flimsily robust eff. solutions (NetM)

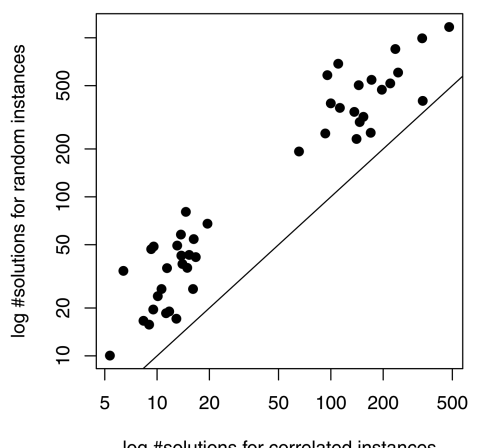

(b) Runtime RL-Flimsily (grid)

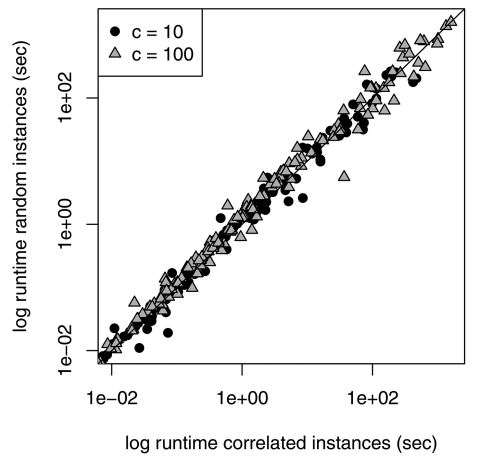

(d) Runtime RL-Flimsily (NetM)

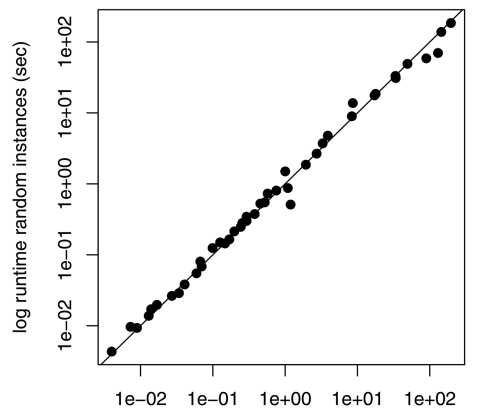

log runtime correlated instances (sec)

F I G U RE 7 Comparing the number of flimsily robust efficient solutions (a) and RL-Flimsily runtimes (b) between grid network instances with correlated and random scenarios (based on averages for $k=2$ ); similarly for NetMaker instances in (c) and (d) (also based on averages). The straight line indicates where instances would have equal numbers of solutions and runtimes, respectively

scenarios (points are close to the line), despite more solutions for random scenarios. This is likely due to similar numbers of efficient solutions found for each scenario, which, in the correlated case, are often the same solution, whereas they are more likely to be distinct solutions in the random case.

Similarly, comparing the plots in Figure 5, it is apparent that the runtime of EL-Flimsily tends to be much lower for correlated NetMaker instances than for random NetMaker instances, as explained in Section 5.2.2. We do not observe this for RL-Flimsily. In Figure 7c,d this is investigated in more detail, similar to Figure 7a,b for grid instances. Again, the average results over all instances with the same parameters are shown. Figure 7d shows runtimes of RL-Flimsily, which tend to be similar for random and correlated instances, even though the number of solutions tends to be higher for random instances, as shown in Figure 7c.

\subsection{5 | Number of robust efficient solutions}

There generally are many flimsily robust efficient solutions, and fewer highly robust efficient solutions.

We note that grid network instances with random scenarios in our experiments do generally not have any highly robust efficient solutions for $k=2,3$, see Tables A11-A14, whereas instances with $k=4$ tend to have a few, mainly for $r=2$ (Tables A15, A16). For grid network instances with correlated scenarios more highly robust efficient solutions are found, see Tables A3-A9, as a solution that is efficient in one scenario is more likely to also be efficient in another (correlated) scenario. This effect is stronger for $c=100$, when compared to $c=10$, leading to more highly robust efficient solutions when $c=100$. In

https://doi.org/10.1002/net.21815 
${ }^{22} \perp$ WILEY— RATTH Eт AL.

$\begin{array}{ll}\text { (a) No of flimsily robust eff. solutions (grid) } & \text { (b) No of highly robust eff. solutions (grid) }\end{array}$
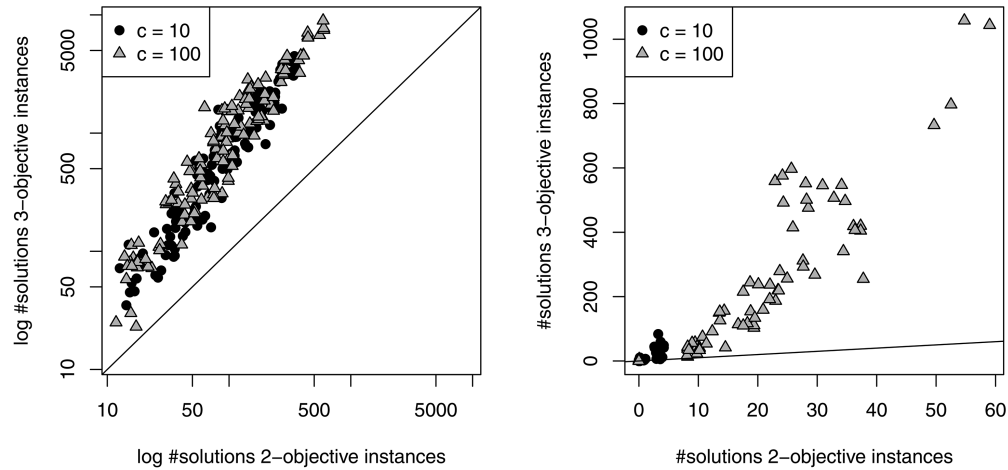

F I G U R E 8 Comparing the number of flimsily (a) and highly (b) robust efficient solutions of correlated and random grid network instances with 2 and 3 objectives (both plots are based on averages for $k=2$ ). The straight line indicates where instances would have equal numbers of solutions

addition, it can be observed that the number of highly robust efficient solutions increases as the number of objectives increases, and that it tends to be higher for fewer scenarios.

As instance size, number of scenarios, and number of objectives increase, the number of flimsily robust solutions found also increases. Figure 8a shows that instances with the same parameters $h, w, r$ with $k=2$ objectives (horizontal axis) and $k=3$ objectives (vertical axis) clearly have more solutions for $k=3$. Figure $8 \mathrm{~b}$ illustrates the increase in highly robust efficient solutions found for $k=3$, again compared to $k=2$.

Our results also show that problem instances become more challenging as their size increases, that is as $h$ and $w$ increase. On closer inspection wider networks are more challenging than higher networks. For example, instances with $h=20, w=30$ have more flimsily robust efficient solutions and longer runtimes than instances with $h=30, w=20$. Narrow and high networks tend to have shorter paths and fewer flimsily robust efficient paths as paths tend to dominate each other more. Wide networks, on the other hand, have longer and more flimsily robust efficient paths as there are more possible ways of traversing the network on paths that do not dominate each other.

Random NetMaker instances also tend to have few highly robust efficient solutions, in particular for only two objectives, where often no highly robust efficient solution exists (see Tables A23, A24). Correlated instances, however, tend to have more highly robust efficient solutions, since an efficient path w.r.t. one scenario is much more likely to be efficient w.r.t. the other scenarios as well, if the edge costs in all scenarios are similar. As for grid network instances, NetMaker instances with three objectives generally have more flimsily and highly robust efficient solutions than instances with only two objectives.

\section{3 | Finding point-based and set-based minmax robust efficient solutions}

EL-PB and EL-SB are, already for small matrices, demanding in terms of runtime and memory usage. A RAM limit of 14 GB did only allow the solution of instances with very small networks. The memory usage and runtime increase rapidly with increasing number of scenarios and/or objectives.

In addition the memory usage and runtimes for instances with the same number of objectives and scenarios and the same network structure differ greatly. An important factor is the number of prediction matrices used in the algorithm, since the number of constructed labels relies heavily on it, which we demonstrate based on a grid network with $h=w=2, k=2$ and $r=2$. The integer edge cost components are randomly chosen between 1 and $c$ from a discrete uniform distribution, where $c$ lies between 2 and 12. For each $c \in\{2, \ldots, 12\}$, ten random instances are created. Figure 9 shows the runtime of EL-PB in relation to the number of prediction matrices for this $2 \times 2$ grid network with $r=2$ and $k=2$. The runtimes of EL-SB show the same trend and are omitted here.

From the theory we know that the number of considered prediction matrices depends on the number of objectives and scenarios and on the lower and upper bounds $A_{i, j}^{\min }$ and $A_{i, j}^{\max }$. Hence, in addition to the number of objectives and scenarios, also the lower and upper bounds play a critical role regarding the runtime (and memory usage) of EL-PB and EL-SB. Since we look

https://doi.org/10.1002/net.21815 
RAITH ET AL.

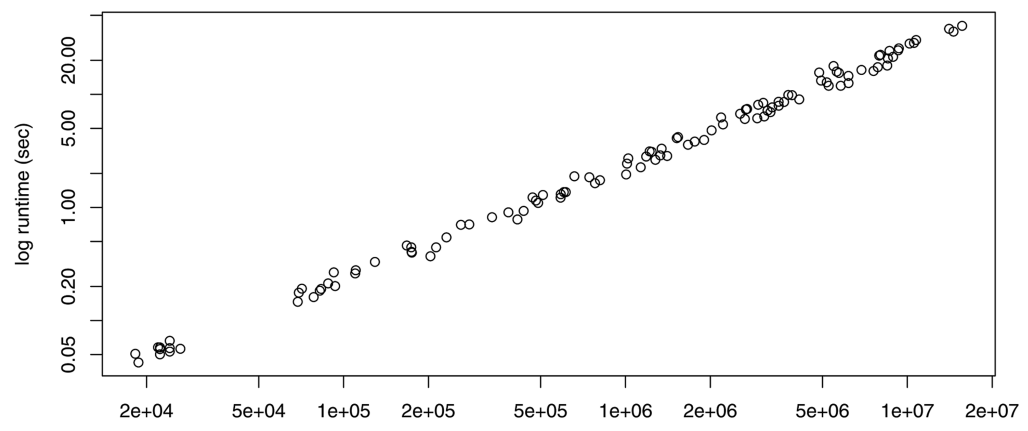

log number of prediction matrices

F I G U R E 9 Runtime of EL-PB for several instances of edge costs for a grid network with $h=w=2, k=2$, and $r=2$, in relation to the number of prediction matrices produced for this instance during execution of the algorithm

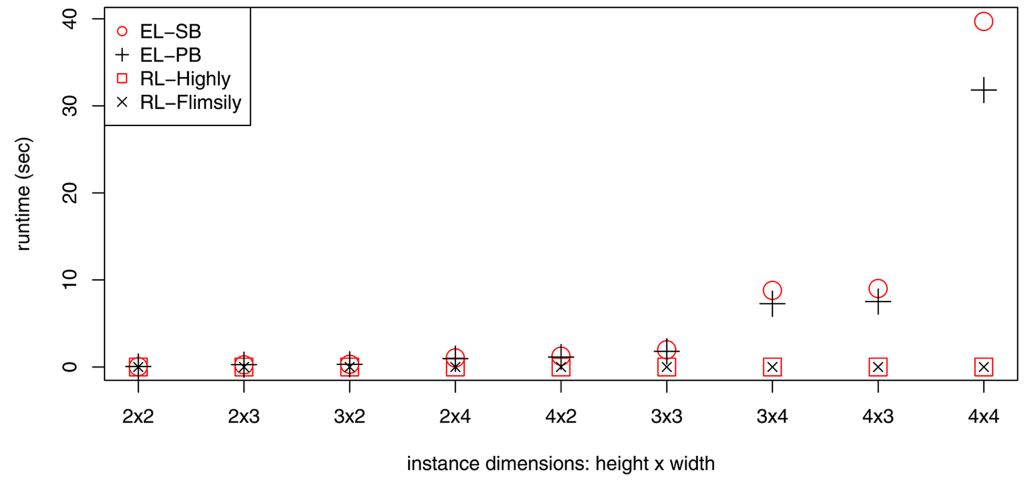

F I G U RE 10 Runtime for grid network instances with two objectives and two scenarios (average of 10 randomly generated instances for each network size with cost components in $\{1,2\}$ ) [Color figure can be viewed at wileyonlinelibrary.com]

for the labels with prediction matrix 0 at node $t$, the lower bounds cannot be chosen higher than 0 . However, the upper bounds depend on the $|V|-1$ maximal edge costs, and thus on the maximal possible edge cost $c$.

As a consequence, to be able to compare networks of different sizes we consider $k=2$ objectives, $r=2$ scenarios and edge costs chosen randomly from $\{1,2\}$ (uniformly distributed). Figure 10 shows the average runtime of ten random instances each for different sizes of grid networks with width and height between 2 and 4. One can see that the average runtimes of EL-PB and EL-SB increase significantly with the size of the network, even for the small networks considered here. Instances with grid networks of width and height larger than 4 could not be solved due to memory capacities. In comparison, the time needed to find flimsily or highly robust efficient solutions increases much more slowly. Further, the runtime of EL-SB is higher and increases faster than the runtime of EL-PB. This can be explained by the complexity of the comparison procedure: to check whether a pair of label costs is in $R^{\text {set }}$ takes more time than to check whether it is in $R^{\text {point }}$.

\section{4 | Summary}

In summary, it is challenging to identify robust efficient solutions even for small to medium sized problem instances, in particular point-based and set-based minmax robust efficient solutions. An increase in the number of scenarios and objectives considered, as well as the size of the network, is associated with an increase in runtime. In case of the algorithms EL-SB and EL-PB, the values of the edge cost components also influence the runtime significantly. The experiments on instances with flimsily and highly robust efficiency show that it is preferable to use the class of repeated labeling algorithms for grid and many NetMaker instances, while extended labeling algorithms sometimes perform better for NetMaker networks with correlated scenarios.

https://doi.org/10.1002/net.21815 
${ }^{24} \perp$ WILEY- RAITH вт AL.

\section{6 | CONCLUSION}

In this article, we have investigated whether and how a generic label correcting algorithm for the multi-objective shortest path problem can be extended to find robust efficient solutions for the multi-objective uncertain shortest path problem. We have introduced algorithms to find robust efficient solutions for several popular concepts of robust efficiency, which can be classified into extended and repeated labeling algorithms. We compared their performance experimentally on several instances of grid networks and NetMaker networks and observed that the repeated labeling algorithms are often, but not always, faster than the extended labeling algorithms. We observed that in particular finding minmax robust efficient solutions is challenging even for small networks and few scenarios and objectives.

Therefore, investigating possible accelerations of the algorithms seems worthwhile, for example, by reducing the number of prediction matrices with the help of better upper bounds on the longest paths. More efficient ways to store and evaluate the information about the prediction matrices, than constructing one label per matrix, are also of interest.

There exists a great number of further concepts of robust efficiency, for example, lightly robust efficiency [24, 26, 39] and hull-based minmax robust efficiency [6]. The conditions for using the generic label correcting algorithm and the methods to extend it, as presented in this article, can also be useful when other concepts are considered.

The algorithm for the multi-objective problem that we have extended for the multi-objective uncertain case, is a generic algorithm with label selection. Our extended algorithms still include the label selection step. It would be of interest which label selection methods are best suited for the algorithms introduced in this article. In addition, the ideas presented to extend the label correcting algorithm with label selection might also be applicable to other labeling algorithms. Further research could also include possible extensions of other methods to solve the multi-objective or the robust shortest path problem.

In robust optimization, PRO (Pareto robust optimal) solutions are of interest (see [22] for single-objective, [26] for biobjective problems and [7] for general multi-objective problems). PRO robust efficient solutions are solutions which are multi-scenario efficient and robust efficient w.r.t. some other concept at the same time. To find PRO robust efficient solutions, the approach given in [26] for bi-objective problems with uncertainty in only one objective can be extended to several uncertain objectives: First, one finds a complete set of multi-scenario efficient solutions, then these solutions are filtered to obtain the PRO robust efficient solutions. In comparison to the filtering procedure given in [26], filtering is much more time consuming for several uncertain objectives. Therefore, efficient filtering methods are of interest. In addition, pruning techniques would be useful, for example, as proposed in [32], where a multi-objective label correcting algorithm is used to find solutions of the single-objective minmax problem.

\section{ACKNOWLEDGMENTS}

Part of the implementations was done by Fabian Chlumsky-Harttmann. Lisa Thom was supported by DFG RTG 1703 "Resource Efficiency in Interorganizational Networks".

\section{ORCID}

Andrea Raith (1) http://orcid.org/0000-0002-0417-2972

Marie Schmidt (1D) http://orcid.org/0000-0001-9563-9955

Anita Schöbel (iD http://orcid.org/0000-0002-9306-5529

Lisa Thom (10 http://orcid.org/0000-0002-8589-6453

\section{REFERENCES}

[1] H. Aissi, C. Bazgan, and D. Vanderpooten, Min-max and min-max regret versions of combinatorial optimization problems: A survey, Eur. J. Oper. Res. 197 (2009), 427-438.

[2] R. Bellman, On a routing problem, Q. Appl. Math. 16 (1958), 87-90.

[3] H.P. Benson, On a domination property for vector maximization with respect to cones, J. Optim. Theory Appl. 39 (1983), $125-132$.

[4] D. Bertsimas and M. Sim, Robust discrete optimization and network flows, Math. Program. 98 (2003), 49-71.

[5] G.R. Bitran, Linear multiple objective problems with interval coefficients, Manag. Sci. 26 (1980), 694-706.

[6] R. Bokrantz and A. Fredriksson, Necessary and sufficient conditions for Pareto efficiency in robust multiobjective optimization, Eur. J. Oper. Res. 262 (2017), 682-692.

https://doi.org/10.1002/net.21815 
RAITH ET AL.

[7] M. Botte and A. Schöbel, Dominance for multi-objective robust optimization concepts, Tech. report 2016-8, Preprint-Reihe, Institut für Numerische und Angewandte Mathematik, Universität Göttingen, 2016.

[8] T.A. Brown and R.E. Strauch, Dynamic programming in multiplicative lattices, J. Math. Anal. Appl. 12 (1965), 364-370.

[9] J. Brumbaugh-Smith and D. Shier, An empirical investigation of some bicriterion shortest path algorithms, Eur. J. Oper. Res. 43 (1989), $216-224$.

[10] A. Candia-Véjar, E. Álvarez-Miranda, and N. Maculan, Minmax regret combinatorial optimization problems: An algorithmic perspective, RAIRO Oper. Res. 45 (2011), 101-129.

[11] W.M. Carlyle and R.K. Wood, Near-shortest and $k$-shortest simple paths, Networks 46 (2005), 98-109.

[12] H.W. Corley and I.D. Moon, Shortest paths in networks with vector weights, J. Optim. Theory Appl. 46 (1985), 79-86.

[13] L.C. Dias and J.N. Clìmaco, Shortest path problems with partial information: Models and algorithms for detecting dominance, Eur. J. Oper. Res. 121 (2000), 16-31.

[14] E.W. Dijkstra, A note on two problems in connexion with graphs, Numer. Math. 1 (1959), 269-271.

[15] T. Dokka and M. Goerigk, Experimental evaluation of uncertainty sets for robust shortest path problem, 17th Workshop on Algorithmic Approaches for Transportation Modelling, Optimization, and Systems (ATMOS2017) (G. D’Angelo and T. Dollevoet, eds.), OASIcs OpenAccess Ser. Inform., vol. 59, Schloss Dagstuhl. Leibniz-Zent. Inform., 2017, pp. 16:1-16:13.

[16] M. Ehrgott, Multicriteria Optimization, Springer, Berlin, 2005.

[17] M. Ehrgott, J. Ide, and A. Schöbel, Minmax robustness for multi-objective optimization problems, Eur. J. Oper. Res. 239 (2014), 17-31.

[18] G. Fadel et al., Multi-criteria multi-scenario approaches in the design of vehicles, 6th World Congresses of Structural and Multidisciplinary Optimization, Rio de Janeiro, Brazil, 2005.

[19] V. Gabrel and C. Murat, Robust shortest path problems, Annales du LAMSADE N 7 (2007), pp. 71-93.

[20] F. Guerriero and R. Musmanno, Label correcting methods to solve multicriteria shortest path problems, J. Optim. Theory Appl. 111 (2001), 589-613.

[21] M.I. Henig, The principle of optimality in dynamic programming with returns in partially ordered sets, Math. Oper. Res. 10 (1985), $462-470$.

[22] D.A. Iancu and N. Trichakis, Pareto efficiency in robust optimization, Manag. Sci. 60 (2013), 130-147.

[23] J. Ide et al., The relationship between multi-objective robustness concepts and set valued optimization, Fixed Point Theory Appl. 2014 (2014), 83.

[24] J. Ide and A. Schöbel, Robustness for uncertain multi-objective optimization: A survey and analysis of different concepts, OR Spectr. 38 (2016), $235-271$.

[25] A. Kasperski and P. Zieliński, Robust discrete optimization under discrete and interval uncertainty: A survey, Robustness Analysis in Decision Aiding, Optimization, and Analytics (M. Doumpos, C. Zopounidis, and E. Grigoroudis, eds.), International Series in Operations Research \& Management Science, Springer, Cham, 2016, pp. 113-143.

[26] K. Kuhn et al., Bi-objective robust optimisation, Eur. J. Oper. Res. 252 (2016), 418-431.

[27] D. Kuroiwa and G.M. Lee, On robust multiobjective optimization, Vietnam J. Math. 40 (2012), 305-317.

[28] E.Q.V. Martins, On a multicriteria shortest path problem, Eur. J. Oper. Res. 16 (1984), 236-245.

[29] L.G. Mitten, Composition principles for synthesis of optimal multistage processes, Oper. Res. 12 (1964), 610-619.

[30] R. Montemanni and L.M. Gambardella, The robust shortest path problem with interval data via Benders decomposition, 4OR 3 (2005), 315-328.

[31] T.L. Morin, Monotonicity and the principle of optimality, J. Math. Anal. Appl. 88 (1982), 665-674.

[32] I. Murthy and S. Her, Solving min-max shortest-path problems on a network, Naval Res. Logist. 39 (1992), 669-683.

[33] J.M. Paixão and J.L. Santos, Labeling methods for the general case of the multi-objective shortest path problem - a computational study, Computational Intelligence and Decision Making (A. Madureira, C. Reis, and V. Marques, eds.), Springer, Dordrecht, 2013 , pp. 489-502.

[34] M. Pascoal and M. Resende, The minmax regret robust shortest path problem in a finite multi-scenario model, Appl. Math. Comput. 241 (2014), $88-111$.

[35] P. Perny and O. Spanjaard, A preference-based approach to spanning trees and shortest paths problems, Eur. J. Oper. Res. 162 (2005), 584-601.

[36] R Core Team, R: A Language and Environment for Statistical Computing, R Foundation for Statistical Computing, Vienna, Austria, 2015.

https://doi.org/10.1002/net.21815 
[37] A. Raith and M. Ehrgott, A comparison of solution strategies for biobjective shortest path problems, Comput. Oper. Res. 36 (2009), 1299-1331.

[38] A. Raith et al., Multi-objective minmax robust combinatorial optimization with cardinality-constrained uncertainty, Eur. J. Oper. Res. 267 (2018), 628-642

[39] A. Schöbel, Generalized light robustness and the trade-off between robustness and nominal quality, Math. Methods Oper. Res. 80 (2014), $161-191$.

[40] A.J.V. Skriver and K.A. Andersen, A label correcting approach for solving bicriterion shortest-path problems, Comput. Oper. Res. 27 (2000), 507-524.

[41] M. M. Wiecek and G. M. Dranichak, Robust multiobjective optimization for decision making under uncertainty and conflict, Optimization Challenges in Complex, Networked and Risky Systems (A. Gupta and A. Capponi, eds.), INFORMS Tutorials in Operations Research (J.C. Smith, ed.), INFORMS, Catonsville, MD, 2016, pp. 84-114.

[42] G. Yu and J. Yang, On the robust shortest path problem, Comput. Oper. Res. 25 (1998), 457-468.

How to cite this article: Raith A, Schmidt M, Schöbel A, Thom L. Extensions of labeling algorithms for multi-objective uncertain shortest path problems. NETWORKS. 2018;00:1-43. https://doi.org/10.1002/net.21815

https://doi.org/10.1002/net.21815 
RAITH ET AL.

APPENDIX: RESULT TABLES

TA B LE A1 Grid instances with two objectives $(k=2)$, correlated scenarios and $c=10$, flimsily robust efficiency, 20 instances for each set of parameters

\begin{tabular}{|c|c|c|c|c|c|c|c|c|c|c|c|c|c|c|c|}
\hline & \multirow[b]{2}{*}{$|V|$} & \multirow[b]{2}{*}{$|E|$} & \multirow[b]{2}{*}{$\boldsymbol{h}$} & \multirow[b]{2}{*}{$w$} & \multirow[b]{2}{*}{$r$} & \multirow[b]{2}{*}{$c$} & \multicolumn{3}{|c|}{ EL-Flimsily } & \multicolumn{3}{|c|}{ RL-Flimsily } & sols & & \\
\hline & & & & & & & $\overline{\min }$ & $\max$ & $\overline{\text { avg }}$ & $\overline{\min }$ & $\max$ & $\overline{\operatorname{avg}}$ & $\overline{\min }$ & $\max$ & $\overline{\text { avg }}$ \\
\hline 1 & 103 & 380 & 10 & 10 & 2 & 10 & 0.00 & 0.00 & 0.00 & 0.00 & 0.00 & 0.00 & 4 & 22 & 12.65 \\
\hline 3 & 103 & 380 & 10 & 10 & 4 & 10 & 0.01 & 0.01 & 0.01 & 0.00 & 0.00 & 0.00 & 12 & 34 & 19.60 \\
\hline 5 & 103 & 380 & 10 & 10 & 6 & 10 & 0.01 & 0.04 & 0.02 & 0.00 & 0.01 & 0.01 & 8 & 43 & 27.75 \\
\hline 7 & 103 & 380 & 10 & 10 & 8 & 10 & 0.02 & 0.05 & 0.03 & 0.01 & 0.01 & 0.01 & 14 & 56 & 35.80 \\
\hline 9 & 203 & 760 & 10 & 20 & 2 & 10 & 0.02 & 0.04 & 0.03 & 0.01 & 0.02 & 0.01 & 22 & 42 & 30.95 \\
\hline 11 & 203 & 760 & 10 & 20 & 4 & 10 & 0.07 & 0.15 & 0.11 & 0.01 & 0.05 & 0.03 & 33 & 74 & 53.45 \\
\hline 13 & 203 & 760 & 10 & 20 & 6 & 10 & 0.07 & 0.45 & 0.28 & 0.02 & 0.06 & 0.04 & 43 & 140 & 87.05 \\
\hline 15 & 203 & 760 & 10 & 20 & 8 & 10 & 0.20 & 0.77 & 0.43 & 0.03 & 0.10 & 0.06 & 59 & 173 & 94.70 \\
\hline 17 & 303 & 1140 & 10 & 30 & 2 & 10 & 0.09 & 0.19 & 0.14 & 0.03 & 0.11 & 0.07 & 40 & 76 & 56.85 \\
\hline 19 & 303 & 1140 & 10 & 30 & 4 & 10 & 0.30 & 0.96 & 0.59 & 0.08 & 0.26 & 0.14 & 61 & 145 & 100.90 \\
\hline 21 & 303 & 1140 & 10 & 30 & 6 & 10 & 0.69 & 2.64 & 1.52 & 0.07 & 0.24 & 0.14 & 86 & 222 & 150.60 \\
\hline 23 & 303 & 1140 & 10 & 30 & 8 & 10 & 1.74 & 5.16 & 3.01 & 0.12 & 0.28 & 0.18 & 143 & 260 & 199.60 \\
\hline 25 & 403 & 1520 & 10 & 40 & 2 & 10 & 0.19 & 0.79 & 0.42 & 0.04 & 0.16 & 0.10 & 39 & 131 & 74.65 \\
\hline 27 & 403 & 1520 & 10 & 40 & 4 & 10 & 0.82 & 3.10 & 1.76 & 0.12 & 0.35 & 0.19 & 95 & 213 & 146.00 \\
\hline 29 & 403 & 1520 & 10 & 40 & 6 & 10 & 2.39 & 9.89 & 5.11 & 0.19 & 0.50 & 0.31 & 179 & 276 & 231.50 \\
\hline 31 & 403 & 1520 & 10 & 40 & 8 & 10 & 4.85 & 21.50 & 12.98 & 0.22 & 0.72 & 0.47 & 167 & 451 & 318.55 \\
\hline 33 & 203 & 780 & 20 & 10 & 2 & 10 & 0.01 & 0.01 & 0.01 & 0.00 & 0.01 & 0.00 & 5 & 23 & 14.35 \\
\hline 35 & 203 & 780 & 20 & 10 & 4 & 10 & 0.01 & 0.03 & 0.02 & 0.01 & 0.01 & 0.01 & 10 & 41 & 24.85 \\
\hline 37 & 203 & 780 & 20 & 10 & 6 & 10 & 0.03 & 0.06 & 0.04 & 0.01 & 0.02 & 0.01 & 16 & 51 & 35.10 \\
\hline 39 & 203 & 780 & 20 & 10 & 8 & 10 & 0.05 & 0.10 & 0.07 & 0.01 & 0.03 & 0.02 & 15 & 69 & 41.80 \\
\hline 41 & 403 & 1560 & 20 & 20 & 2 & 10 & 0.06 & 0.12 & 0.07 & 0.02 & 0.04 & 0.02 & 23 & 48 & 33.70 \\
\hline 43 & 403 & 1560 & 20 & 20 & 4 & 10 & 0.14 & 0.53 & 0.26 & 0.03 & 0.09 & 0.05 & 44 & 110 & 67.25 \\
\hline 45 & 403 & 1560 & 20 & 20 & 6 & 10 & 0.28 & 0.89 & 0.55 & 0.04 & 0.09 & 0.07 & 41 & 128 & 81.80 \\
\hline 47 & 403 & 1560 & 20 & 20 & 8 & 10 & 0.56 & 1.93 & 1.08 & 0.07 & 0.16 & 0.10 & 54 & 137 & 103.65 \\
\hline 49 & 603 & 2340 & 20 & 30 & 2 & 10 & 0.22 & 0.64 & 0.32 & 0.06 & 0.16 & 0.08 & 38 & 88 & 55.50 \\
\hline 51 & 603 & 2340 & 20 & 30 & 4 & 10 & 0.96 & 1.84 & 1.42 & 0.12 & 0.21 & 0.17 & 71 & 132 & 106.05 \\
\hline 53 & 603 & 2340 & 20 & 30 & 6 & 10 & 2.04 & 6.26 & 3.73 & 0.21 & 0.47 & 0.29 & 106 & 262 & 167.85 \\
\hline 55 & 603 & 2340 & 20 & 30 & 8 & 10 & 4.23 & 15.76 & 8.00 & 0.25 & 0.55 & 0.39 & 144 & 310 & 217.25 \\
\hline 57 & 803 & 3120 & 20 & 40 & 2 & 10 & 0.66 & 1.75 & 1.05 & 0.14 & 0.40 & 0.25 & 47 & 110 & 83.80 \\
\hline 59 & 803 & 3120 & 20 & 40 & 4 & 10 & 2.74 & 6.81 & 4.68 & 0.34 & 0.74 & 0.51 & 112 & 252 & 168.95 \\
\hline 61 & 803 & 3120 & 20 & 40 & 6 & 10 & 9.38 & 28.94 & 14.67 & 0.58 & 1.08 & 0.80 & 171 & 351 & 272.15 \\
\hline 63 & 803 & 3120 & 20 & 40 & 8 & 10 & 17.70 & 44.44 & 26.97 & 0.75 & 1.61 & 1.02 & 231 & 449 & 322.10 \\
\hline 65 & 303 & 1180 & 30 & 10 & 2 & 10 & 0.01 & 0.02 & 0.01 & 0.00 & 0.01 & 0.01 & 3 & 23 & 15.00 \\
\hline 67 & 303 & 1180 & 30 & 10 & 4 & 10 & 0.02 & 0.05 & 0.03 & 0.01 & 0.01 & 0.01 & 10 & 42 & 24.25 \\
\hline 69 & 303 & 1180 & 30 & 10 & 6 & 10 & 0.05 & 0.09 & 0.07 & 0.01 & 0.02 & 0.02 & 22 & 44 & 33.65 \\
\hline 71 & 303 & 1180 & 30 & 10 & 8 & 10 & 0.07 & 0.13 & 0.10 & 0.02 & 0.03 & 0.02 & 26 & 51 & 39.55 \\
\hline 73 & 603 & 2360 & 30 & 20 & 2 & 10 & 0.09 & 0.19 & 0.12 & 0.03 & 0.05 & 0.04 & 18 & 51 & 35.75 \\
\hline 75 & 603 & 2360 & 30 & 20 & 4 & 10 & 0.25 & 0.52 & 0.41 & 0.05 & 0.09 & 0.07 & 43 & 97 & 61.10 \\
\hline 77 & 603 & 2360 & 30 & 20 & 6 & 10 & 0.60 & 1.76 & 1.00 & 0.09 & 0.17 & 0.12 & 36 & 135 & 88.45 \\
\hline 79 & 603 & 2360 & 30 & 20 & 8 & 10 & 1.18 & 3.42 & 1.80 & 0.12 & 0.22 & 0.16 & 45 & 167 & 116.10 \\
\hline 81 & 903 & 3540 & 30 & 30 & 2 & 10 & 0.34 & 0.75 & 0.51 & 0.09 & 0.19 & 0.13 & 39 & 82 & 54.80 \\
\hline 83 & 903 & 3540 & 30 & 30 & 4 & 10 & 1.59 & 2.70 & 2.18 & 0.20 & 0.34 & 0.28 & 84 & 145 & 111.35 \\
\hline 85 & 903 & 3540 & 30 & 30 & 6 & 10 & 4.13 & 8.61 & 5.50 & 0.33 & 0.48 & 0.41 & 94 & 218 & 165.40 \\
\hline 87 & 903 & 3540 & 30 & 30 & 8 & 10 & 7.26 & 15.39 & 10.80 & 0.47 & 0.74 & 0.59 & 133 & 264 & 215.70 \\
\hline 89 & 1203 & 4720 & 30 & 40 & 2 & 10 & 1.04 & 2.28 & 1.55 & 0.23 & 0.46 & 0.33 & 56 & 110 & 81.65 \\
\hline 91 & 1203 & 4720 & 30 & 40 & 4 & 10 & 5.83 & 11.03 & 8.60 & 0.57 & 1.11 & 0.84 & 140 & 248 & 188.70 \\
\hline 93 & 1203 & 4720 & 30 & 40 & 6 & 10 & 15.52 & 29.63 & 22.92 & 1.04 & 1.67 & 1.28 & 187 & 360 & 271.10 \\
\hline 95 & 1203 & 4720 & 30 & 40 & 8 & 10 & 31.81 & 63.96 & 48.12 & 1.15 & 2.72 & 1.77 & 255 & 458 & 349.45 \\
\hline 97 & 403 & 1580 & 40 & 10 & 2 & 10 & 0.01 & 0.02 & 0.02 & 0.01 & 0.01 & 0.01 & 9 & 26 & 15.85 \\
\hline 99 & 403 & 1580 & 40 & 10 & 4 & 10 & 0.04 & 0.06 & 0.05 & 0.01 & 0.02 & 0.02 & 11 & 40 & 26.10 \\
\hline 101 & 403 & 1580 & 40 & 10 & 6 & 10 & 0.07 & 0.12 & 0.09 & 0.02 & 0.04 & 0.03 & 17 & 46 & 31.55 \\
\hline 103 & 403 & 1580 & 40 & 10 & 8 & 10 & 0.10 & 0.18 & 0.14 & 0.03 & 0.04 & 0.03 & 31 & 67 & 43.60 \\
\hline 105 & 803 & 3160 & 40 & 20 & 2 & 10 & 0.11 & 0.23 & 0.15 & 0.03 & 0.07 & 0.05 & 17 & 58 & 36.70 \\
\hline 107 & 803 & 3160 & 40 & 20 & 4 & 10 & 0.48 & 0.73 & 0.57 & 0.09 & 0.12 & 0.10 & 41 & 93 & 71.15 \\
\hline 109 & 803 & 3160 & 40 & 20 & 6 & 10 & 1.03 & 1.70 & 1.35 & 0.14 & 0.20 & 0.17 & 71 & 128 & 91.65 \\
\hline 111 & 803 & 3160 & 40 & 20 & 8 & 10 & 1.76 & 2.98 & 2.30 & 0.18 & 0.27 & 0.21 & 67 & 149 & 111.30 \\
\hline 113 & 1203 & 4740 & 40 & 30 & 2 & 10 & 0.51 & 1.21 & 0.74 & 0.12 & 0.31 & 0.18 & 35 & 90 & 61.70 \\
\hline 115 & 1203 & 4740 & 40 & 30 & 4 & 10 & 1.65 & 3.63 & 2.83 & 0.24 & 0.44 & 0.36 & 68 & 147 & 111.10 \\
\hline 117 & 1203 & 4740 & 40 & 30 & 6 & 10 & 5.48 & 10.65 & 7.69 & 0.46 & 0.82 & 0.61 & 101 & 194 & 159.20 \\
\hline 119 & 1203 & 4740 & 40 & 30 & 8 & 10 & 10.39 & 19.77 & 15.31 & 0.59 & 0.97 & 0.81 & 106 & 286 & 217.55 \\
\hline 121 & 1603 & 6320 & 40 & 40 & 2 & 10 & 1.32 & 2.91 & 2.25 & 0.30 & 0.62 & 0.51 & 55 & 116 & 86.90 \\
\hline 123 & 1603 & 6320 & 40 & 40 & 4 & 10 & 7.94 & 17.02 & 10.63 & 0.86 & 1.69 & 1.10 & 121 & 263 & 179.45 \\
\hline 125 & 1603 & 6320 & 40 & 40 & 6 & 10 & 23.53 & 39.65 & 30.27 & 1.40 & 2.08 & 1.73 & 162 & 339 & 261.65 \\
\hline 127 & 1603 & 6320 & 40 & 40 & 8 & 10 & 38.21 & 88.07 & 58.58 & 1.52 & 3.24 & 2.20 & 251 & 526 & 337.95 \\
\hline
\end{tabular}

https://doi.org/10.1002/net. 21815 
A.2. Extensions of Labeling Algorithms

${ }^{28}$ WI LEY

RAITH ET AL.

T A B LE A2 Grid instances with two objectives $(k=2)$, correlated scenarios and $c=100$, flimsily robust efficiency, 20 instances for each set of parameters

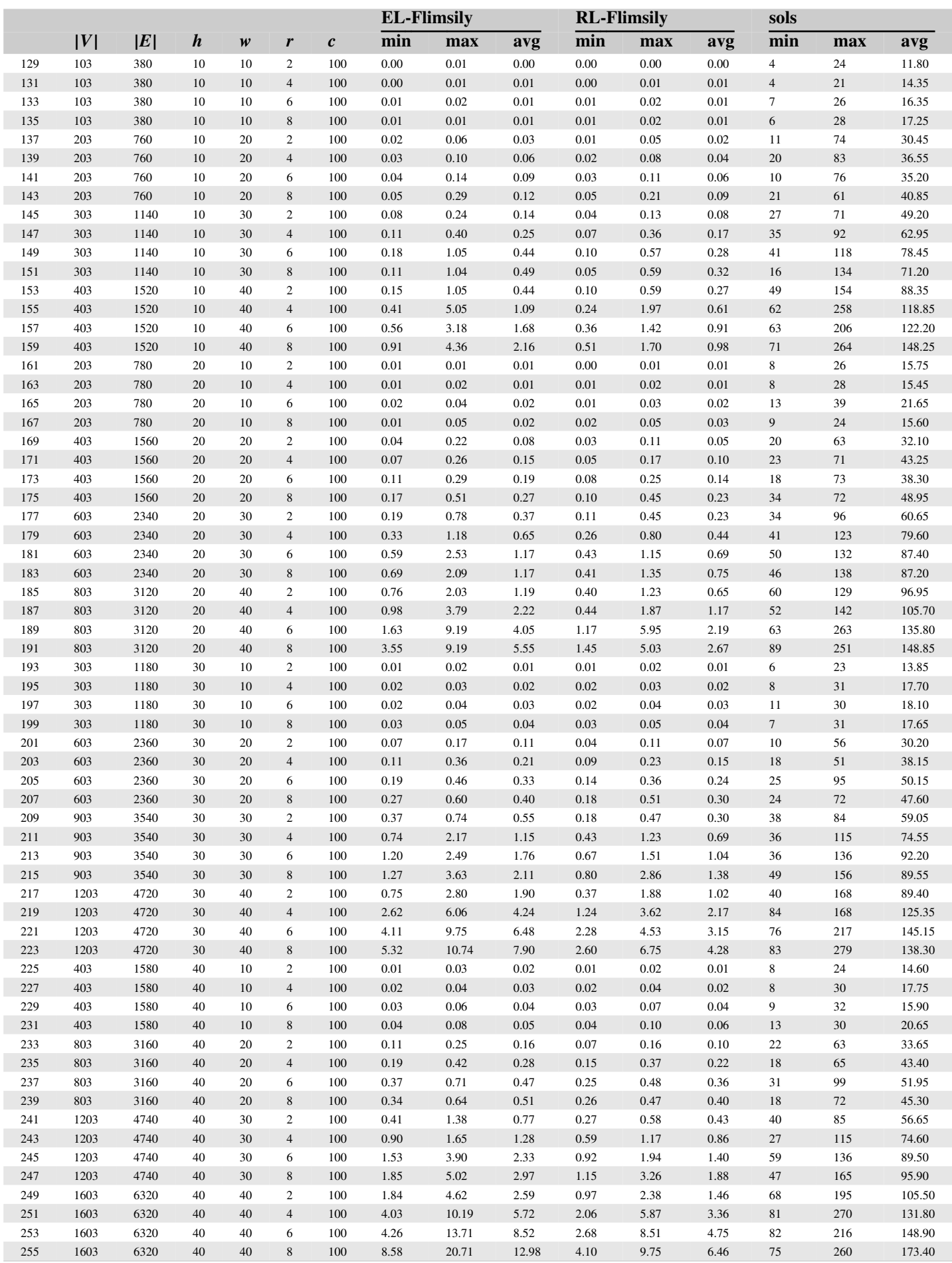

https://doi.org/10.1002/net.21815 
RAITH ET AL.

WILEY

T A B LE A3 Grid instances with two objectives $(k=2)$, correlated scenarios and $c=10$, highly robust efficiency, 20 instances for each set of parameters

\begin{tabular}{|c|c|c|c|c|c|c|c|c|c|c|c|c|c|c|c|}
\hline & \multirow[b]{2}{*}{$|\boldsymbol{V}|$} & \multirow[b]{2}{*}{$|E|$} & \multirow[b]{2}{*}{$\boldsymbol{h}$} & \multirow[b]{2}{*}{$w$} & \multirow[b]{2}{*}{$r$} & \multirow[b]{2}{*}{$c$} & \multicolumn{3}{|c|}{ EL-Highly } & \multicolumn{3}{|c|}{ RL-Highly } & sols & & \\
\hline & & & & & & & $\overline{\min }$ & $\max$ & $\overline{\text { avg }}$ & $\overline{\min }$ & $\max$ & $\overline{\text { avg }}$ & $\overline{\min }$ & $\max$ & $\overline{\text { avg }}$ \\
\hline 1 & 103 & 380 & 10 & 10 & 2 & 10 & 0.00 & 0.01 & 0.00 & 0.00 & 0.00 & 0.00 & 1 & 9 & 3.60 \\
\hline 3 & 103 & 380 & 10 & 10 & 4 & 10 & 0.01 & 0.01 & 0.01 & 0.00 & 0.00 & 0.00 & 0 & 3 & 1.10 \\
\hline 5 & 103 & 380 & 10 & 10 & 6 & 10 & 0.01 & 0.04 & 0.02 & 0.00 & 0.01 & 0.01 & 0 & 3 & 0.80 \\
\hline 7 & 103 & 380 & 10 & 10 & 8 & 10 & 0.02 & 0.05 & 0.03 & 0.01 & 0.01 & 0.01 & 0 & 3 & 0.40 \\
\hline 9 & 203 & 760 & 10 & 20 & 2 & 10 & 0.02 & 0.04 & 0.03 & 0.01 & 0.02 & 0.01 & 0 & 6 & 2.90 \\
\hline 11 & 203 & 760 & 10 & 20 & 4 & 10 & 0.07 & 0.15 & 0.11 & 0.01 & 0.04 & 0.02 & 0 & 3 & 0.80 \\
\hline 13 & 203 & 760 & 10 & 20 & 6 & 10 & 0.07 & 0.46 & 0.28 & 0.02 & 0.06 & 0.04 & 0 & 1 & 0.15 \\
\hline 15 & 203 & 760 & 10 & 20 & 8 & 10 & 0.20 & 0.79 & 0.43 & 0.03 & 0.07 & 0.04 & 0 & 1 & 0.10 \\
\hline 17 & 303 & 1140 & 10 & 30 & 2 & 10 & 0.09 & 0.19 & 0.14 & 0.02 & 0.06 & 0.04 & 0 & 9 & 4.20 \\
\hline 19 & 303 & 1140 & 10 & 30 & 4 & 10 & 0.30 & 0.98 & 0.60 & 0.05 & 0.12 & 0.08 & 0 & 1 & 0.15 \\
\hline 21 & 303 & 1140 & 10 & 30 & 6 & 10 & 0.71 & 2.64 & 1.55 & 0.06 & 0.23 & 0.13 & 0 & 0 & 0.00 \\
\hline 23 & 303 & 1140 & 10 & 30 & 8 & 10 & 1.81 & 5.23 & 3.11 & 0.11 & 0.25 & 0.17 & 0 & 0 & 0.00 \\
\hline 25 & 403 & 1520 & 10 & 40 & 2 & 10 & 0.19 & 0.74 & 0.42 & 0.04 & 0.16 & 0.10 & 0 & 7 & 2.70 \\
\hline 27 & 403 & 1520 & 10 & 40 & 4 & 10 & 0.82 & 3.17 & 1.78 & 0.11 & 0.35 & 0.19 & 0 & 1 & 0.20 \\
\hline 29 & 403 & 1520 & 10 & 40 & 6 & 10 & 2.48 & 10.35 & 5.29 & 0.19 & 0.51 & 0.31 & 0 & 0 & 0.00 \\
\hline 31 & 403 & 1520 & 10 & 40 & 8 & 10 & 4.93 & 22.29 & 13.44 & 0.22 & 0.70 & 0.46 & 0 & 0 & 0.00 \\
\hline 33 & 203 & 780 & 20 & 10 & 2 & 10 & 0.01 & 0.01 & 0.01 & 0.00 & 0.00 & 0.00 & 1 & 8 & 3.60 \\
\hline 35 & 203 & 780 & 20 & 10 & 4 & 10 & 0.01 & 0.03 & 0.02 & 0.01 & 0.01 & 0.01 & 0 & 4 & 0.90 \\
\hline 37 & 203 & 780 & 20 & 10 & 6 & 10 & 0.03 & 0.06 & 0.04 & 0.01 & 0.02 & 0.01 & 0 & 1 & 0.25 \\
\hline 39 & 203 & 780 & 20 & 10 & 8 & 10 & 0.05 & 0.10 & 0.07 & 0.01 & 0.02 & 0.02 & 0 & 1 & 0.10 \\
\hline 41 & 403 & 1560 & 20 & 20 & 2 & 10 & 0.06 & 0.12 & 0.07 & 0.02 & 0.04 & 0.02 & 1 & 9 & 3.30 \\
\hline 43 & 403 & 1560 & 20 & 20 & 4 & 10 & 0.14 & 0.52 & 0.27 & 0.03 & 0.09 & 0.05 & 0 & 2 & 0.30 \\
\hline 45 & 403 & 1560 & 20 & 20 & 6 & 10 & 0.29 & 0.91 & 0.57 & 0.04 & 0.09 & 0.07 & 0 & 1 & 0.10 \\
\hline 47 & 403 & 1560 & 20 & 20 & 8 & 10 & 0.58 & 1.97 & 1.10 & 0.06 & 0.16 & 0.10 & 0 & 1 & 0.10 \\
\hline 49 & 603 & 2340 & 20 & 30 & 2 & 10 & 0.22 & 0.64 & 0.33 & 0.06 & 0.16 & 0.08 & 0 & 7 & 2.95 \\
\hline 51 & 603 & 2340 & 20 & 30 & 4 & 10 & 0.97 & 1.89 & 1.44 & 0.12 & 0.21 & 0.17 & 0 & 2 & 0.35 \\
\hline 53 & 603 & 2340 & 20 & 30 & 6 & 10 & 2.10 & 6.39 & 3.84 & 0.21 & 0.47 & 0.29 & 0 & 1 & 0.05 \\
\hline 55 & 603 & 2340 & 20 & 30 & 8 & 10 & 4.39 & 16.13 & 8.50 & 0.25 & 0.55 & 0.38 & 0 & 0 & 0.00 \\
\hline 57 & 803 & 3120 & 20 & 40 & 2 & 10 & 0.65 & 1.77 & 1.06 & 0.14 & 0.39 & 0.25 & 0 & 12 & 3.65 \\
\hline 59 & 803 & 3120 & 20 & 40 & 4 & 10 & 2.81 & 6.82 & 4.78 & 0.33 & 0.73 & 0.51 & 0 & 3 & 0.20 \\
\hline 61 & 803 & 3120 & 20 & 40 & 6 & 10 & 9.61 & 29.69 & 14.98 & 0.56 & 1.09 & 0.80 & 0 & 0 & 0.00 \\
\hline 63 & 803 & 3120 & 20 & 40 & 8 & 10 & 18.01 & 45.68 & 27.57 & 0.73 & 1.60 & 1.02 & 0 & 0 & 0.00 \\
\hline 65 & 303 & 1180 & 30 & 10 & 2 & 10 & 0.01 & 0.02 & 0.01 & 0.00 & 0.01 & 0.01 & 0 & 5 & 2.95 \\
\hline 67 & 303 & 1180 & 30 & 10 & 4 & 10 & 0.02 & 0.05 & 0.03 & 0.01 & 0.01 & 0.01 & 0 & 3 & 1.10 \\
\hline 69 & 303 & 1180 & 30 & 10 & 6 & 10 & 0.05 & 0.09 & 0.07 & 0.01 & 0.02 & 0.02 & 0 & 2 & 0.50 \\
\hline 71 & 303 & 1180 & 30 & 10 & 8 & 10 & 0.07 & 0.13 & 0.10 & 0.02 & 0.03 & 0.02 & 0 & 1 & 0.20 \\
\hline 73 & 603 & 2360 & 30 & 20 & 2 & 10 & 0.09 & 0.19 & 0.12 & 0.03 & 0.05 & 0.04 & 0 & 9 & 4.20 \\
\hline 75 & 603 & 2360 & 30 & 20 & 4 & 10 & 0.26 & 0.54 & 0.42 & 0.05 & 0.09 & 0.07 & 0 & 1 & 0.15 \\
\hline 77 & 603 & 2360 & 30 & 20 & 6 & 10 & 0.62 & 1.94 & 1.03 & 0.08 & 0.17 & 0.12 & 0 & 2 & 0.25 \\
\hline 79 & 603 & 2360 & 30 & 20 & 8 & 10 & 1.23 & 3.44 & 1.86 & 0.12 & 0.22 & 0.16 & 0 & 2 & 0.15 \\
\hline 81 & 903 & 3540 & 30 & 30 & 2 & 10 & 0.34 & 0.76 & 0.52 & 0.08 & 0.19 & 0.13 & 0 & 7 & 2.65 \\
\hline 83 & 903 & 3540 & 30 & 30 & 4 & 10 & 1.69 & 2.77 & 2.21 & 0.20 & 0.33 & 0.28 & 0 & 1 & 0.15 \\
\hline 85 & 903 & 3540 & 30 & 30 & 6 & 10 & 4.20 & 8.71 & 5.54 & 0.33 & 0.48 & 0.41 & 0 & 1 & 0.05 \\
\hline 87 & 903 & 3540 & 30 & 30 & 8 & 10 & 7.24 & 15.50 & 10.88 & 0.47 & 0.74 & 0.58 & 0 & 0 & 0.00 \\
\hline 89 & 1203 & 4720 & 30 & 40 & 2 & 10 & 1.05 & 2.23 & 1.55 & 0.23 & 0.45 & 0.33 & 0 & 9 & 2.60 \\
\hline 91 & 1203 & 4720 & 30 & 40 & 4 & 10 & 5.59 & 11.24 & 8.70 & 0.57 & 1.12 & 0.86 & 0 & 1 & 0.05 \\
\hline 93 & 1203 & 4720 & 30 & 40 & 6 & 10 & 15.87 & 29.90 & 23.33 & 1.01 & 1.66 & 1.27 & 0 & 0 & 0.00 \\
\hline 95 & 1203 & 4720 & 30 & 40 & 8 & 10 & 32.53 & 65.46 & 49.26 & 1.15 & 2.82 & 1.75 & 0 & 0 & 0.00 \\
\hline 97 & 403 & 1580 & 40 & 10 & 2 & 10 & 0.01 & 0.02 & 0.02 & 0.01 & 0.01 & 0.01 & 2 & 6 & 3.95 \\
\hline 99 & 403 & 1580 & 40 & 10 & 4 & 10 & 0.04 & 0.06 & 0.05 & 0.01 & 0.02 & 0.02 & 0 & 3 & 0.95 \\
\hline 101 & 403 & 1580 & 40 & 10 & 6 & 10 & 0.07 & 0.13 & 0.09 & 0.02 & 0.03 & 0.02 & 0 & 1 & 0.60 \\
\hline 103 & 403 & 1580 & 40 & 10 & 8 & 10 & 0.10 & 0.18 & 0.15 & 0.02 & 0.04 & 0.03 & 0 & 2 & 0.25 \\
\hline 105 & 803 & 3160 & 40 & 20 & 2 & 10 & 0.11 & 0.23 & 0.16 & 0.03 & 0.07 & 0.05 & 0 & 7 & 3.15 \\
\hline 107 & 803 & 3160 & 40 & 20 & 4 & 10 & 0.48 & 0.73 & 0.58 & 0.09 & 0.12 & 0.10 & 0 & 3 & 0.25 \\
\hline 109 & 803 & 3160 & 40 & 20 & 6 & 10 & 0.95 & 1.70 & 1.37 & 0.14 & 0.21 & 0.17 & 0 & 2 & 0.15 \\
\hline 111 & 803 & 3160 & 40 & 20 & 8 & 10 & 1.78 & 2.96 & 2.34 & 0.18 & 0.28 & 0.21 & 0 & 0 & 0.00 \\
\hline 113 & 1203 & 4740 & 40 & 30 & 2 & 10 & 0.50 & 1.22 & 0.73 & 0.12 & 0.31 & 0.18 & 0 & 9 & 3.40 \\
\hline 115 & 1203 & 4740 & 40 & 30 & 4 & 10 & 1.68 & 3.74 & 2.89 & 0.24 & 0.45 & 0.36 & 0 & 3 & 0.35 \\
\hline 117 & 1203 & 4740 & 40 & 30 & 6 & 10 & 5.68 & 11.10 & 7.88 & 0.46 & 0.84 & 0.60 & 0 & 1 & 0.05 \\
\hline 119 & 1203 & 4740 & 40 & 30 & 8 & 10 & 10.19 & 20.34 & 15.68 & 0.61 & 0.99 & 0.80 & 0 & 0 & 0.00 \\
\hline 121 & 1603 & 6320 & 40 & 40 & 2 & 10 & 1.33 & 2.86 & 2.27 & 0.29 & 0.60 & 0.51 & 0 & 7 & 3.25 \\
\hline 123 & 1603 & 6320 & 40 & 40 & 4 & 10 & 8.09 & 16.87 & 10.71 & 0.86 & 1.66 & 1.09 & 0 & 1 & 0.10 \\
\hline 125 & 1603 & 6320 & 40 & 40 & 6 & 10 & 23.93 & 40.16 & 30.81 & 1.37 & 2.03 & 1.71 & 0 & 1 & 0.05 \\
\hline 127 & 1603 & 6320 & 40 & 40 & 8 & 10 & 38.91 & 90.21 & 59.36 & 1.51 & 3.13 & 2.23 & 0 & 0 & 0.00 \\
\hline
\end{tabular}

https://doi.org/10.1002/net.21815 
A.2. Extensions of Labeling Algorithms

${ }^{30}$ WI LEY

RAITH ET AL.

T A B L E A4 Grid instances with two objectives $(k=2)$, correlated scenarios and $c=100$, highly robust efficiency, 20 instances for each set of parameters

\begin{tabular}{|c|c|c|c|c|c|c|c|c|c|c|c|c|c|c|c|}
\hline & \multirow[b]{2}{*}{$|V|$} & \multirow[b]{2}{*}{$|E|$} & \multirow[b]{2}{*}{$\boldsymbol{h}$} & \multirow[b]{2}{*}{$w$} & \multirow[b]{2}{*}{$r$} & \multirow[b]{2}{*}{$c$} & \multicolumn{3}{|c|}{ EL-Highly } & \multicolumn{3}{|c|}{ RL-Highly } & sols & & \\
\hline & & & & & & & $\overline{\min }$ & $\max$ & $\overline{\text { avg }}$ & $\overline{\min }$ & $\max$ & $\overline{\text { avg }}$ & $\overline{\min }$ & $\max$ & $\overline{\text { avg }}$ \\
\hline 129 & 103 & 380 & 10 & 10 & 2 & 100 & 0.00 & 0.01 & 0.00 & 0.00 & 0.00 & 0.00 & 4 & 18 & 9.75 \\
\hline 131 & 103 & 380 & 10 & 10 & 4 & 100 & 0.00 & 0.01 & 0.01 & 0.00 & 0.01 & 0.01 & 4 & 11 & 8.45 \\
\hline 133 & 103 & 380 & 10 & 10 & 6 & 100 & 0.01 & 0.02 & 0.01 & 0.01 & 0.02 & 0.01 & 5 & 13 & 9.50 \\
\hline 135 & 103 & 380 & 10 & 10 & 8 & 100 & 0.01 & 0.02 & 0.01 & 0.01 & 0.02 & 0.01 & 4 & 12 & 8.20 \\
\hline 137 & 203 & 760 & 10 & 20 & 2 & 100 & 0.02 & 0.07 & 0.03 & 0.01 & 0.05 & 0.02 & 7 & 39 & 20.90 \\
\hline 139 & 203 & 760 & 10 & 20 & 4 & 100 & 0.03 & 0.11 & 0.06 & 0.02 & 0.08 & 0.04 & 10 & 32 & 17.55 \\
\hline 141 & 203 & 760 & 10 & 20 & 6 & 100 & 0.04 & 0.14 & 0.09 & 0.03 & 0.11 & 0.07 & 5 & 22 & 13.50 \\
\hline 143 & 203 & 760 & 10 & 20 & 8 & 100 & 0.05 & 0.29 & 0.12 & 0.05 & 0.21 & 0.09 & 7 & 20 & 14.55 \\
\hline 145 & 303 & 1140 & 10 & 30 & 2 & 100 & 0.09 & 0.23 & 0.14 & 0.04 & 0.13 & 0.09 & 19 & 55 & 34.40 \\
\hline 147 & 303 & 1140 & 10 & 30 & 4 & 100 & 0.11 & 0.39 & 0.24 & 0.07 & 0.33 & 0.17 & 15 & 33 & 25.65 \\
\hline 149 & 303 & 1140 & 10 & 30 & 6 & 100 & 0.18 & 1.01 & 0.44 & 0.11 & 0.59 & 0.29 & 13 & 32 & 23.70 \\
\hline 151 & 303 & 1140 & 10 & 30 & 8 & 100 & 0.12 & 1.02 & 0.49 & 0.05 & 0.58 & 0.33 & 8 & 27 & 18.70 \\
\hline 153 & 403 & 1520 & 10 & 40 & 2 & 100 & 0.15 & 1.04 & 0.43 & 0.10 & 0.58 & 0.26 & 32 & 94 & 49.65 \\
\hline 155 & 403 & 1520 & 10 & 40 & 4 & 100 & 0.41 & 5.01 & 1.07 & 0.20 & 1.90 & 0.57 & 24 & 61 & 34.70 \\
\hline 157 & 403 & 1520 & 10 & 40 & 6 & 100 & 0.56 & 3.22 & 1.67 & 0.27 & 1.41 & 0.85 & 4 & 40 & 28.05 \\
\hline 159 & 403 & 1520 & 10 & 40 & 8 & 100 & 0.91 & 4.40 & 2.18 & 0.53 & 1.84 & 1.02 & 17 & 36 & 24.95 \\
\hline 161 & 203 & 780 & 20 & 10 & 2 & 100 & 0.01 & 0.02 & 0.01 & 0.00 & 0.01 & 0.01 & 7 & 21 & 12.35 \\
\hline 163 & 203 & 780 & 20 & 10 & 4 & 100 & 0.01 & 0.02 & 0.01 & 0.01 & 0.02 & 0.01 & 5 & 14 & 9.45 \\
\hline 165 & 203 & 780 & 20 & 10 & 6 & 100 & 0.01 & 0.04 & 0.02 & 0.01 & 0.03 & 0.02 & 6 & 15 & 9.80 \\
\hline 167 & 203 & 780 & 20 & 10 & 8 & 100 & 0.01 & 0.04 & 0.02 & 0.01 & 0.04 & 0.02 & 5 & 13 & 8.10 \\
\hline 169 & 403 & 1560 & 20 & 20 & 2 & 100 & 0.04 & 0.20 & 0.08 & 0.03 & 0.11 & 0.05 & 13 & 45 & 23.00 \\
\hline 171 & 403 & 1560 & 20 & 20 & 4 & 100 & 0.07 & 0.25 & 0.14 & 0.05 & 0.18 & 0.10 & 13 & 28 & 19.35 \\
\hline 173 & 403 & 1560 & 20 & 20 & 6 & 100 & 0.12 & 0.30 & 0.19 & 0.08 & 0.22 & 0.15 & 3 & 22 & 13.65 \\
\hline 175 & 403 & 1560 & 20 & 20 & 8 & 100 & 0.17 & 0.51 & 0.27 & 0.10 & 0.37 & 0.22 & 9 & 25 & 16.70 \\
\hline 177 & 603 & 2340 & 20 & 30 & 2 & 100 & 0.19 & 0.87 & 0.38 & 0.11 & 0.54 & 0.25 & 23 & 52 & 37.75 \\
\hline 179 & 603 & 2340 & 20 & 30 & 4 & 100 & 0.33 & 1.18 & 0.65 & 0.25 & 0.80 & 0.43 & 19 & 48 & 29.60 \\
\hline 181 & 603 & 2340 & 20 & 30 & 6 & 100 & 0.57 & 2.59 & 1.16 & 0.43 & 1.07 & 0.68 & 8 & 32 & 24.30 \\
\hline 183 & 603 & 2340 & 20 & 30 & 8 & 100 & 0.66 & 2.05 & 1.17 & 0.41 & 1.29 & 0.73 & 10 & 38 & 22.05 \\
\hline 185 & 803 & 3120 & 20 & 40 & 2 & 100 & 0.77 & 1.99 & 1.19 & 0.38 & 1.13 & 0.64 & 28 & 73 & 54.75 \\
\hline 187 & 803 & 3120 & 20 & 40 & 4 & 100 & 0.98 & 3.77 & 2.21 & 0.43 & 1.56 & 1.13 & 22 & 49 & 34.10 \\
\hline 189 & 803 & 3120 & 20 & 40 & 6 & 100 & 1.65 & 9.11 & 4.07 & 1.00 & 4.65 & 2.03 & 19 & 47 & 32.80 \\
\hline 191 & 803 & 3120 & 20 & 40 & 8 & 100 & 3.48 & 9.35 & 5.52 & 1.42 & 4.84 & 2.63 & 13 & 49 & 25.90 \\
\hline 193 & 303 & 1180 & 30 & 10 & 2 & 100 & 0.01 & 0.02 & 0.01 & 0.01 & 0.02 & 0.01 & 6 & 19 & 10.70 \\
\hline 195 & 303 & 1180 & 30 & 10 & 4 & 100 & 0.02 & 0.03 & 0.02 & 0.01 & 0.03 & 0.02 & 6 & 15 & 10.20 \\
\hline 197 & 303 & 1180 & 30 & 10 & 6 & 100 & 0.02 & 0.05 & 0.03 & 0.02 & 0.04 & 0.03 & 5 & 14 & 8.95 \\
\hline 199 & 303 & 1180 & 30 & 10 & 8 & 100 & 0.02 & 0.05 & 0.04 & 0.03 & 0.05 & 0.04 & 3 & 12 & 8.20 \\
\hline 201 & 603 & 2360 & 30 & 20 & 2 & 100 & 0.07 & 0.15 & 0.11 & 0.04 & 0.11 & 0.07 & 9 & 32 & 22.00 \\
\hline 203 & 603 & 2360 & 30 & 20 & 4 & 100 & 0.11 & 0.36 & 0.20 & 0.09 & 0.24 & 0.16 & 13 & 24 & 19.35 \\
\hline 205 & 603 & 2360 & 30 & 20 & 6 & 100 & 0.19 & 0.43 & 0.32 & 0.14 & 0.39 & 0.24 & 13 & 25 & 18.25 \\
\hline 207 & 603 & 2360 & 30 & 20 & 8 & 100 & 0.27 & 0.58 & 0.40 & 0.20 & 0.56 & 0.32 & 4 & 28 & 14.35 \\
\hline 209 & 903 & 3540 & 30 & 30 & 2 & 100 & 0.36 & 0.74 & 0.54 & 0.18 & 0.47 & 0.30 & 25 & 51 & 37.30 \\
\hline 211 & 903 & 3540 & 30 & 30 & 4 & 100 & 0.71 & 2.19 & 1.15 & 0.43 & 1.45 & 0.73 & 19 & 35 & 27.60 \\
\hline 213 & 903 & 3540 & 30 & 30 & 6 & 100 & 1.20 & 2.51 & 1.75 & 0.67 & 1.61 & 1.08 & 16 & 33 & 22.90 \\
\hline 215 & 903 & 3540 & 30 & 30 & 8 & 100 & 1.26 & 3.73 & 2.13 & 0.81 & 1.97 & 1.26 & 9 & 26 & 18.80 \\
\hline 217 & 1203 & 4720 & 30 & 40 & 2 & 100 & 0.75 & 2.78 & 1.91 & 0.38 & 1.83 & 1.03 & 30 & 92 & 52.50 \\
\hline 219 & 1203 & 4720 & 30 & 40 & 4 & 100 & 2.57 & 6.36 & 4.22 & 1.24 & 4.01 & 2.20 & 26 & 48 & 36.10 \\
\hline 221 & 1203 & 4720 & 30 & 40 & 6 & 100 & 4.25 & 9.84 & 6.53 & 2.30 & 5.45 & 3.63 & 19 & 42 & 28.50 \\
\hline 223 & 1203 & 4720 & 30 & 40 & 8 & 100 & 5.28 & 10.82 & 7.92 & 2.54 & 7.54 & 4.36 & 16 & 41 & 28.20 \\
\hline 225 & 403 & 1580 & 40 & 10 & 2 & 100 & 0.01 & 0.02 & 0.02 & 0.01 & 0.02 & 0.01 & 7 & 19 & 11.35 \\
\hline 227 & 403 & 1580 & 40 & 10 & 4 & 100 & 0.02 & 0.05 & 0.03 & 0.02 & 0.04 & 0.03 & 5 & 16 & 10.20 \\
\hline 229 & 403 & 1580 & 40 & 10 & 6 & 100 & 0.03 & 0.07 & 0.04 & 0.03 & 0.06 & 0.04 & 3 & 15 & 8.20 \\
\hline 231 & 403 & 1580 & 40 & 10 & 8 & 100 & 0.04 & 0.08 & 0.05 & 0.04 & 0.07 & 0.06 & 6 & 13 & 8.40 \\
\hline 233 & 803 & 3160 & 40 & 20 & 2 & 100 & 0.13 & 0.25 & 0.16 & 0.08 & 0.14 & 0.11 & 15 & 43 & 23.40 \\
\hline 235 & 803 & 3160 & 40 & 20 & 4 & 100 & 0.19 & 0.42 & 0.29 & 0.15 & 0.36 & 0.23 & 7 & 29 & 19.50 \\
\hline 237 & 803 & 3160 & 40 & 20 & 6 & 100 & 0.37 & 0.75 & 0.48 & 0.29 & 0.46 & 0.37 & 13 & 25 & 17.55 \\
\hline 239 & 803 & 3160 & 40 & 20 & 8 & 100 & 0.35 & 0.62 & 0.51 & 0.25 & 0.53 & 0.42 & 8 & 17 & 13.60 \\
\hline 241 & 1203 & 4740 & 40 & 30 & 2 & 100 & 0.42 & 1.41 & 0.78 & 0.24 & 0.78 & 0.44 & 26 & 49 & 37.30 \\
\hline 243 & 1203 & 4740 & 40 & 30 & 4 & 100 & 0.93 & 1.63 & 1.29 & 0.59 & 1.11 & 0.79 & 15 & 46 & 27.65 \\
\hline 245 & 1203 & 4740 & 40 & 30 & 6 & 100 & 1.56 & 3.82 & 2.34 & 0.91 & 2.15 & 1.36 & 15 & 32 & 23.50 \\
\hline 247 & 1203 & 4740 & 40 & 30 & 8 & 100 & 1.85 & 5.22 & 3.01 & 1.12 & 3.26 & 1.74 & 15 & 29 & 20.10 \\
\hline 249 & 1603 & 6320 & 40 & 40 & 2 & 100 & 1.85 & 4.82 & 2.63 & 0.96 & 2.31 & 1.39 & 36 & 114 & 59.00 \\
\hline 251 & 1603 & 6320 & 40 & 40 & 4 & 100 & 4.08 & 10.29 & 5.79 & 2.06 & 4.72 & 2.89 & 7 & 58 & 36.35 \\
\hline 253 & 1603 & 6320 & 40 & 40 & 6 & 100 & 4.23 & 14.13 & 8.56 & 2.18 & 5.67 & 4.20 & 19 & 41 & 30.90 \\
\hline 255 & 1603 & 6320 & 40 & 40 & 8 & 100 & 8.91 & 21.57 & 13.32 & 3.91 & 9.26 & 6.08 & 11 & 40 & 24.15 \\
\hline
\end{tabular}

https://doi.org/10.1002/net.21815 
A. Publications

RAITH ET AL.

WILEY

TA B LE A5 Grid instances with three objectives $(k=3)$, correlated scenarios and $c=10$

\begin{tabular}{|c|c|c|c|c|c|c|c|c|c|c|c|c|}
\hline & $|V|$ & $|E|$ & $\boldsymbol{h}$ & $w$ & $r$ & $c$ & EL-Flimsily & RL-Flimsily & sols & EL-Highly & RL-Highly & sols \\
\hline 1025 & 103 & 380 & 10 & 10 & 2 & 10 & 0.04 & 0.03 & 72 & 0.02 & 0.01 & 21 \\
\hline 1027 & 103 & 380 & 10 & 10 & 4 & 10 & 0.09 & 0.04 & 96 & 0.07 & 0.02 & 7 \\
\hline 1029 & 103 & 380 & 10 & 10 & 6 & 10 & 0.11 & 0.03 & 69 & 0.10 & 0.03 & 2 \\
\hline 1031 & 103 & 380 & 10 & 10 & 8 & 10 & 0.15 & 0.04 & 105 & 0.15 & 0.04 & 2 \\
\hline 1033 & 203 & 760 & 10 & 20 & 2 & 10 & 0.27 & 0.08 & 155 & 0.26 & 0.09 & 26 \\
\hline 1035 & 203 & 760 & 10 & 20 & 4 & 10 & 1.25 & 0.19 & 240 & 1.20 & 0.20 & 5 \\
\hline 1037 & 203 & 760 & 10 & 20 & 6 & 10 & 3.19 & 0.38 & 283 & 3.07 & 0.38 & 0 \\
\hline 1039 & 203 & 760 & 10 & 20 & 8 & 10 & 6.55 & 0.56 & 510 & 6.14 & 0.56 & 2 \\
\hline 1041 & 303 & 1140 & 10 & 30 & 2 & 10 & 5.56 & 1.64 & 490 & 5.57 & 1.64 & 42 \\
\hline 1043 & 303 & 1140 & 10 & 30 & 4 & 10 & 16.03 & 2.28 & 996 & 16.77 & 2.26 & 1 \\
\hline 1045 & 303 & 1140 & 10 & 30 & 6 & 10 & 60.32 & 2.96 & 1078 & 50.14 & 2.98 & 3 \\
\hline 1047 & 303 & 1140 & 10 & 30 & 8 & 10 & 42.26 & 2.45 & 808 & 43.13 & 2.51 & 1 \\
\hline 1049 & 403 & 1520 & 10 & 40 & 2 & 10 & 21.81 & 5.15 & 634 & 22.33 & 5.30 & 41 \\
\hline 1051 & 403 & 1520 & 10 & 40 & 4 & 10 & 209.11 & 15.87 & 1723 & 202.09 & 16.00 & 9 \\
\hline 1053 & 403 & 1520 & 10 & 40 & 6 & 10 & 416.58 & 14.04 & 2081 & 404.02 & 12.99 & 0 \\
\hline 1055 & 403 & 1520 & 10 & 40 & 8 & 10 & 1018.44 & 29.61 & 4179 & 1005.64 & 24.91 & 1 \\
\hline 1057 & 203 & 780 & 20 & 10 & 2 & 10 & 0.02 & 0.01 & 35 & 0.02 & 0.01 & 6 \\
\hline 1059 & 203 & 780 & 20 & 10 & 4 & 10 & 0.13 & 0.04 & 63 & 0.13 & 0.04 & 3 \\
\hline 1061 & 203 & 780 & 20 & 10 & 6 & 10 & 0.20 & 0.06 & 90 & 0.23 & 0.06 & 3 \\
\hline 1063 & 203 & 780 & 20 & 10 & 8 & 10 & 0.25 & 0.07 & 135 & 0.24 & 0.06 & 2 \\
\hline 1065 & 403 & 1560 & 20 & 20 & 2 & 10 & 1.17 & 0.58 & 209 & 1.19 & 0.47 & 40 \\
\hline 1067 & 403 & 1560 & 20 & 20 & 4 & 10 & 3.51 & 0.74 & 455 & 3.41 & 0.62 & 7 \\
\hline 1069 & 403 & 1560 & 20 & 20 & 6 & 10 & 12.03 & 1.33 & 708 & 12.25 & 1.25 & 0 \\
\hline 1071 & 403 & 1560 & 20 & 20 & 8 & 10 & 15.46 & 1.13 & 544 & 15.60 & 1.07 & 1 \\
\hline 1073 & 603 & 2340 & 20 & 30 & 2 & 10 & 8.78 & 2.16 & 355 & 8.33 & 2.15 & 7 \\
\hline 1075 & 603 & 2340 & 20 & 30 & 4 & 10 & 68.36 & 5.17 & 597 & 51.39 & 5.22 & 5 \\
\hline 1077 & 603 & 2340 & 20 & 30 & 6 & 10 & 137.32 & 8.45 & 1116 & 126.29 & 7.76 & 3 \\
\hline 1079 & 603 & 2340 & 20 & 30 & 8 & 10 & 290.04 & 11.84 & 1173 & 278.94 & 11.09 & 1 \\
\hline 1081 & 803 & 3120 & 20 & 40 & 2 & 10 & 65.09 & 22.59 & 927 & 53.52 & 17.63 & 60 \\
\hline 1083 & 803 & 3120 & 20 & 40 & 4 & 10 & 710.31 & 71.53 & 1740 & 627.12 & 62.86 & 2 \\
\hline 1085 & 803 & 3120 & 20 & 40 & 6 & 10 & 936.21 & 50.42 & 1615 & 822.91 & 46.90 & 2 \\
\hline 1087 & 803 & 3120 & 20 & 40 & 8 & 10 & 2943.12 & 114.73 & 4074 & 2854.50 & 94.95 & 0 \\
\hline 1089 & 303 & 1180 & 30 & 10 & 2 & 10 & 0.10 & 0.04 & 114 & 0.10 & 0.05 & 34 \\
\hline 1091 & 303 & 1180 & 30 & 10 & 4 & 10 & 0.22 & 0.07 & 145 & 0.23 & 0.08 & 7 \\
\hline 1093 & 303 & 1180 & 30 & 10 & 6 & 10 & 0.41 & 0.10 & 112 & 0.42 & 0.11 & 3 \\
\hline 1095 & 303 & 1180 & 30 & 10 & 8 & 10 & 0.76 & 0.14 & 222 & 0.76 & 0.15 & 2 \\
\hline 1097 & 603 & 2360 & 30 & 20 & 2 & 10 & 1.66 & 0.56 & 230 & 2.11 & 0.56 & 49 \\
\hline 1099 & 603 & 2360 & 30 & 20 & 4 & 10 & 8.85 & 1.51 & 422 & 8.66 & 1.54 & 11 \\
\hline 1101 & 603 & 2360 & 30 & 20 & 6 & 10 & 19.84 & 1.96 & 499 & 17.40 & 1.98 & 5 \\
\hline 1103 & 603 & 2360 & 30 & 20 & 8 & 10 & 32.78 & 2.14 & 570 & 29.51 & 2.17 & 1 \\
\hline 1105 & 903 & 3540 & 30 & 30 & 2 & 10 & 14.47 & 4.77 & 443 & 15.74 & 4.80 & 41 \\
\hline 1107 & 903 & 3540 & 30 & 30 & 4 & 10 & 69.30 & 10.08 & 986 & 68.99 & 8.43 & 3 \\
\hline 1109 & 903 & 3540 & 30 & 30 & 6 & 10 & 481.32 & 30.90 & 1513 & 432.96 & 24.93 & 2 \\
\hline 1111 & 903 & 3540 & 30 & 30 & 8 & 10 & 742.98 & 39.42 & 2263 & 778.46 & 31.54 & 0 \\
\hline 1113 & 1203 & 4720 & 30 & 40 & 2 & 10 & 110.40 & 41.12 & 1582 & 113.89 & 36.97 & 45 \\
\hline 1115 & 1203 & 4720 & 30 & 40 & 4 & 10 & 715.42 & 98.19 & 2273 & 751.41 & 87.72 & 1 \\
\hline 1117 & 1203 & 4720 & 30 & 40 & 6 & 10 & 2606.18 & 163.44 & 3579 & 2782.03 & 144.30 & 0 \\
\hline 1119 & 1203 & 4720 & 30 & 40 & 8 & 10 & $>3600.00$ & 194.80 & 3551 & $>3600.00$ & 165.90 & 1 \\
\hline 1121 & 403 & 1580 & 40 & 10 & 2 & 10 & 0.08 & 0.03 & 54 & 0.08 & 0.04 & 12 \\
\hline 1123 & 403 & 1580 & 40 & 10 & 4 & 10 & 0.23 & 0.07 & 60 & 0.27 & 0.08 & 4 \\
\hline 1125 & 403 & 1580 & 40 & 10 & 6 & 10 & 0.64 & 0.15 & 113 & 0.62 & 0.16 & 4 \\
\hline 1127 & 403 & 1580 & 40 & 10 & 8 & 10 & 0.97 & 0.19 & 175 & 0.99 & 0.19 & 2 \\
\hline 1129 & 803 & 3160 & 40 & 20 & 2 & 10 & 1.47 & 0.68 & 179 & 1.54 & 0.53 & 27 \\
\hline 1131 & 803 & 3160 & 40 & 20 & 4 & 10 & 5.48 & 1.22 & 160 & 5.63 & 1.00 & 7 \\
\hline 1133 & 803 & 3160 & 40 & 20 & 6 & 10 & 24.71 & 2.96 & 524 & 28.47 & 2.58 & 1 \\
\hline 1135 & 803 & 3160 & 40 & 20 & 8 & 10 & 46.75 & 4.61 & 715 & 53.54 & 3.77 & 2 \\
\hline 1137 & 1203 & 4740 & 40 & 30 & 2 & 10 & 22.17 & 6.77 & 437 & 23.59 & 6.67 & 44 \\
\hline 1139 & 1203 & 4740 & 40 & 30 & 4 & 10 & 97.76 & 17.14 & 1033 & 106.29 & 15.78 & 5 \\
\hline 1141 & 1203 & 4740 & 40 & 30 & 6 & 10 & 499.83 & 38.67 & 1973 & 535.59 & 34.74 & 0 \\
\hline 1143 & 1203 & 4740 & 40 & 30 & 8 & 10 & 522.77 & 32.17 & 1823 & 566.18 & 25.82 & 2 \\
\hline 1145 & 1603 & 6320 & 40 & 40 & 2 & 10 & 198.73 & 73.84 & 1038 & 217.47 & 71.63 & 84 \\
\hline 1147 & 1603 & 6320 & 40 & 40 & 4 & 10 & 837.58 & 114.30 & 1661 & 898.48 & 99.70 & 3 \\
\hline 1149 & 1603 & 6320 & 40 & 40 & 6 & 10 & 2962.03 & 225.56 & 2900 & 3317.24 & 199.17 & 2 \\
\hline 1151 & 1603 & 6320 & 40 & 40 & 8 & 10 & $>3600.00$ & 189.83 & 3069 & $>3600.00$ & 175.23 & 1 \\
\hline
\end{tabular}

https://doi.org/10.1002/net.21815 
A.2. Extensions of Labeling Algorithms

${ }^{32} \perp$ WILEY

RAITH ET AL.

TA B LE A6 Grid instances with three objectives $(k=3)$, correlated scenarios and $c=100$

\begin{tabular}{|c|c|c|c|c|c|c|c|c|c|c|c|c|}
\hline & $|V|$ & $|E|$ & $\boldsymbol{h}$ & $w$ & $r$ & $c$ & EL-Flimsily & RL-Flimsily & sols & EL-Highly & RL-Highly & sols \\
\hline 1153 & 103 & 380 & 10 & 10 & 2 & 100 & 0.01 & 0.01 & 25 & 0.01 & 0.01 & 23 \\
\hline 1155 & 103 & 380 & 10 & 10 & 4 & 100 & 0.03 & 0.04 & 58 & 0.03 & 0.05 & 39 \\
\hline 1157 & 103 & 380 & 10 & 10 & 6 & 100 & 0.06 & 0.06 & 88 & 0.07 & 0.07 & 52 \\
\hline 1159 & 103 & 380 & 10 & 10 & 8 & 100 & 0.02 & 0.02 & 23 & 0.02 & 0.02 & 13 \\
\hline 1161 & 203 & 760 & 10 & 20 & 2 & 100 & 0.64 & 0.49 & 250 & 0.64 & 0.50 & 159 \\
\hline 1163 & 203 & 760 & 10 & 20 & 4 & 100 & 1.23 & 1.41 & 371 & 1.28 & 1.40 & 215 \\
\hline 1165 & 203 & 760 & 10 & 20 & 6 & 100 & 1.29 & 1.09 & 412 & 1.31 & 1.09 & 150 \\
\hline 1167 & 203 & 760 & 10 & 20 & 8 & 100 & 0.74 & 0.60 & 114 & 0.78 & 0.63 & 42 \\
\hline 1169 & 303 & 1140 & 10 & 30 & 2 & 100 & 5.71 & 5.20 & 478 & 6.06 & 5.09 & 341 \\
\hline 1171 & 303 & 1140 & 10 & 30 & 4 & 100 & 60.49 & 36.72 & 1653 & 70.41 & 36.67 & 597 \\
\hline 1173 & 303 & 1140 & 10 & 30 & 6 & 100 & 22.42 & 15.80 & 736 & 23.18 & 15.84 & 279 \\
\hline 1175 & 303 & 1140 & 10 & 30 & 8 & 100 & 24.33 & 17.33 & 997 & 27.75 & 17.39 & 244 \\
\hline 1177 & 403 & 1520 & 10 & 40 & 2 & 100 & 30.76 & 30.02 & 1211 & 35.43 & 30.37 & 733 \\
\hline 1179 & 403 & 1520 & 10 & 40 & 4 & 100 & 171.00 & 100.59 & 1552 & 199.69 & 100.81 & 497 \\
\hline 1181 & 403 & 1520 & 10 & 40 & 6 & 100 & 399.05 & 213.91 & 2050 & 425.82 & 211.03 & 552 \\
\hline 1183 & 403 & 1520 & 10 & 40 & 8 & 100 & 197.30 & 112.93 & 1202 & 193.04 & 102.14 & 256 \\
\hline 1185 & 203 & 780 & 20 & 10 & 2 & 100 & 0.07 & 0.06 & 113 & 0.07 & 0.10 & 92 \\
\hline 1187 & 203 & 780 & 20 & 10 & 4 & 100 & 0.08 & 0.10 & 81 & 0.08 & 0.10 & 57 \\
\hline 1189 & 203 & 780 & 20 & 10 & 6 & 100 & 0.10 & 0.12 & 74 & 0.10 & 0.11 & 44 \\
\hline 1191 & 203 & 780 & 20 & 10 & 8 & 100 & 0.12 & 0.17 & 30 & 0.12 & 0.13 & 17 \\
\hline 1193 & 403 & 1560 & 20 & 20 & 2 & 100 & 0.98 & 0.95 & 258 & 0.97 & 0.75 & 187 \\
\hline 1195 & 403 & 1560 & 20 & 20 & 4 & 100 & 1.04 & 1.20 & 204 & 1.01 & 0.98 & 103 \\
\hline 1197 & 403 & 1560 & 20 & 20 & 6 & 100 & 1.66 & 2.12 & 320 & 1.65 & 1.52 & 126 \\
\hline 1199 & 403 & 1560 & 20 & 20 & 8 & 100 & 4.16 & 3.93 & 279 & 4.12 & 3.04 & 114 \\
\hline 1201 & 603 & 2340 & 20 & 30 & 2 & 100 & 8.45 & 7.01 & 359 & 7.43 & 6.43 & 255 \\
\hline 1203 & 603 & 2340 & 20 & 30 & 4 & 100 & 35.34 & 25.79 & 613 & 28.75 & 22.23 & 268 \\
\hline 1205 & 603 & 2340 & 20 & 30 & 6 & 100 & 234.84 & 153.17 & 1558 & 196.22 & 136.94 & 492 \\
\hline 1207 & 603 & 2340 & 20 & 30 & 8 & 100 & 91.63 & 84.96 & 932 & 82.78 & 64.63 & 237 \\
\hline 1209 & 803 & 3120 & 20 & 40 & 2 & 100 & 158.33 & 148.63 & 1530 & 150.82 & 138.40 & 1058 \\
\hline 1211 & 803 & 3120 & 20 & 40 & 4 & 100 & 302.19 & 203.66 & 1543 & 281.19 & 200.48 & 547 \\
\hline 1213 & 803 & 3120 & 20 & 40 & 6 & 100 & 942.37 & 507.00 & 1939 & 838.62 & 515.52 & 507 \\
\hline 1215 & 803 & 3120 & 20 & 40 & 8 & 100 & 402.88 & 261.81 & 1982 & 383.54 & 231.58 & 415 \\
\hline 1217 & 303 & 1180 & 30 & 10 & 2 & 100 & 0.08 & 0.06 & 90 & 0.07 & 0.06 & 75 \\
\hline 1219 & 303 & 1180 & 30 & 10 & 4 & 100 & 0.16 & 0.17 & 83 & 0.15 & 0.20 & 41 \\
\hline 1221 & 303 & 1180 & 30 & 10 & 6 & 100 & 0.16 & 0.18 & 118 & 0.15 & 0.18 & 56 \\
\hline 1223 & 303 & 1180 & 30 & 10 & 8 & 100 & 0.17 & 0.21 & 73 & 0.17 & 0.18 & 38 \\
\hline 1225 & 603 & 2360 & 30 & 20 & 2 & 100 & 2.29 & 1.67 & 264 & 2.26 & 1.67 & 192 \\
\hline 1227 & 603 & 2360 & 30 & 20 & 4 & 100 & 3.03 & 2.89 & 255 & 2.98 & 2.90 & 111 \\
\hline 1229 & 603 & 2360 & 30 & 20 & 6 & 100 & 4.69 & 3.84 & 313 & 4.58 & 3.81 & 117 \\
\hline 1231 & 603 & 2360 & 30 & 20 & 8 & 100 & 10.11 & 8.08 & 476 & 10.02 & 7.93 & 156 \\
\hline 1233 & 903 & 3540 & 30 & 30 & 2 & 100 & 30.81 & 24.92 & 544 & 30.04 & 24.55 & 420 \\
\hline 1235 & 903 & 3540 & 30 & 30 & 4 & 100 & 39.94 & 36.10 & 836 & 37.08 & 35.23 & 312 \\
\hline 1237 & 903 & 3540 & 30 & 30 & 6 & 100 & 155.08 & 113.83 & 1613 & 143.78 & 111.96 & 559 \\
\hline 1239 & 903 & 3540 & 30 & 30 & 8 & 100 & 149.24 & 96.78 & 576 & 137.10 & 95.40 & 154 \\
\hline 1241 & 1203 & 4720 & 30 & 40 & 2 & 100 & 343.62 & 267.94 & 1276 & 313.32 & 260.41 & 797 \\
\hline 1243 & 1203 & 4720 & 30 & 40 & 4 & 100 & 316.15 & 292.15 & 1132 & 302.56 & 283.07 & 419 \\
\hline 1245 & 1203 & 4720 & 30 & 40 & 6 & 100 & 1088.94 & 615.35 & 1631 & 1000.14 & 639.58 & 476 \\
\hline 1247 & 1203 & 4720 & 30 & 40 & 8 & 100 & 821.87 & 542.08 & 1763 & 805.75 & 525.14 & 501 \\
\hline 1249 & 403 & 1580 & 40 & 10 & 2 & 100 & 0.11 & 0.07 & 75 & 0.10 & 0.08 & 54 \\
\hline 1251 & 403 & 1580 & 40 & 10 & 4 & 100 & 0.19 & 0.21 & 81 & 0.19 & 0.20 & 34 \\
\hline 1253 & 403 & 1580 & 40 & 10 & 6 & 100 & 0.32 & 0.32 & 75 & 0.32 & 0.33 & 44 \\
\hline 1255 & 403 & 1580 & 40 & 10 & 8 & 100 & 0.27 & 0.29 & 86 & 0.26 & 0.30 & 34 \\
\hline 1257 & 803 & 3160 & 40 & 20 & 2 & 100 & 1.77 & 1.24 & 272 & 1.77 & 1.24 & 220 \\
\hline 1259 & 803 & 3160 & 40 & 20 & 4 & 100 & 7.20 & 5.62 & 248 & 6.40 & 5.63 & 134 \\
\hline 1261 & 803 & 3160 & 40 & 20 & 6 & 100 & 5.50 & 5.28 & 209 & 5.42 & 5.16 & 110 \\
\hline 1263 & 803 & 3160 & 40 & 20 & 8 & 100 & 9.62 & 8.17 & 571 & 9.52 & 10.02 & 153 \\
\hline 1265 & 1203 & 4740 & 40 & 30 & 2 & 100 & 36.65 & 32.04 & 611 & 35.65 & 31.83 & 405 \\
\hline 1267 & 1203 & 4740 & 40 & 30 & 4 & 100 & 65.64 & 65.62 & 861 & 61.00 & 65.43 & 293 \\
\hline 1269 & 1203 & 4740 & 40 & 30 & 6 & 100 & 105.86 & 93.21 & 709 & 102.93 & 93.33 & 219 \\
\hline 1271 & 1203 & 4740 & 40 & 30 & 8 & 100 & 261.07 & 179.21 & 1012 & 248.83 & 181.48 & 238 \\
\hline 1273 & 1603 & 6320 & 40 & 40 & 2 & 100 & 437.69 & 429.50 & 1693 & 499.49 & 420.51 & 1044 \\
\hline 1275 & 1603 & 6320 & 40 & 40 & 4 & 100 & 359.67 & 313.99 & 967 & 365.49 & 325.76 & 407 \\
\hline 1277 & 1603 & 6320 & 40 & 40 & 6 & 100 & 1692.17 & 1011.59 & 2367 & 1746.84 & 1008.35 & 546 \\
\hline 1279 & 1603 & 6320 & 40 & 40 & 8 & 100 & 1821.40 & 1340.75 & 2584 & 1872.05 & 1377.21 & 576 \\
\hline
\end{tabular}

https://doi.org/10.1002/net.21815 
RAITH ET AL.

WILEY $\perp^{\perp^{33}}$

T A B L E A7 Grid instances with four objectives $(k=4)$, correlated scenarios and $c=10$

\begin{tabular}{|c|c|c|c|c|c|c|c|c|c|c|c|c|}
\hline & $|V|$ & $|E|$ & $h$ & $w$ & $r$ & $c$ & EL-Flimsily & RL-Flimsily & sols & EL-Highly & RL-Highly & sols \\
\hline 2050 & 103 & 380 & 10 & 10 & 2 & 10 & 0.08 & 0.07 & 145 & 0.08 & 0.04 & 52 \\
\hline 2052 & 103 & 380 & 10 & 10 & 4 & 10 & 0.16 & 0.06 & 196 & 0.15 & 0.05 & 19 \\
\hline 2054 & 203 & 760 & 10 & 20 & 2 & 10 & 1.39 & 0.47 & 409 & 1.29 & 0.50 & 72 \\
\hline 2056 & 203 & 760 & 10 & 20 & 4 & 10 & 11.37 & 2.27 & 748 & 11.36 & 2.04 & 43 \\
\hline 2058 & 303 & 1140 & 10 & 30 & 2 & 10 & 44.86 & 14.18 & 1857 & 47.83 & 13.19 & 251 \\
\hline 2060 & 303 & 1140 & 10 & 30 & 4 & 10 & 375.28 & 36.62 & 3020 & 372.86 & 36.48 & 60 \\
\hline 2062 & 203 & 780 & 20 & 10 & 2 & 10 & 0.09 & 0.04 & 76 & 0.09 & 0.04 & 22 \\
\hline 2064 & 203 & 780 & 20 & 10 & 4 & 10 & 0.42 & 0.13 & 171 & 0.41 & 0.13 & 8 \\
\hline 2066 & 403 & 1560 & 20 & 20 & 2 & 10 & 6.97 & 2.34 & 687 & 6.91 & 2.28 & 129 \\
\hline 2068 & 403 & 1560 & 20 & 20 & 4 & 10 & 37.94 & 4.96 & 1197 & 37.86 & 4.95 & 32 \\
\hline 2070 & 603 & 2340 & 20 & 30 & 2 & 10 & 210.21 & 59.32 & 2557 & 202.54 & 59.25 & 347 \\
\hline 2072 & 603 & 2340 & 20 & 30 & 4 & 10 & 3272.60 & 419.15 & 8058 & 3074.41 & 425.33 & 95 \\
\hline 2074 & 303 & 1180 & 30 & 10 & 2 & 10 & 0.20 & 0.08 & 117 & 0.20 & 0.09 & 44 \\
\hline 2076 & 303 & 1180 & 30 & 10 & 4 & 10 & 0.83 & 0.27 & 261 & 0.81 & 0.23 & 17 \\
\hline 2078 & 603 & 2360 & 30 & 20 & 2 & 10 & 23.83 & 8.54 & 827 & 24.71 & 8.57 & 125 \\
\hline 2080 & 603 & 2360 & 30 & 20 & 4 & 10 & 104.13 & 15.94 & 1544 & 103.93 & 16.18 & 44 \\
\hline 2082 & 903 & 3540 & 30 & 30 & 2 & 10 & 261.00 & 81.94 & 1487 & 256.21 & 82.26 & 197 \\
\hline 2084 & 903 & 3540 & 30 & 30 & 4 & 10 & 3538.64 & 471.57 & 5264 & 3411.47 & 470.48 & 56 \\
\hline
\end{tabular}

T A B LE A8 Grid instances with four objectives $(k=4)$, correlated scenarios and $c=100$

\begin{tabular}{|c|c|c|c|c|c|c|c|c|c|c|c|c|}
\hline & $|V|$ & $|E|$ & $h$ & $w$ & $r$ & $c$ & EL-Flimsily & RL-Flimsily & sols & EL-Highly & RL-Highly & sols \\
\hline 2086 & 103 & 380 & 10 & 10 & 2 & 100 & 0.07 & 0.05 & 145 & 0.07 & 0.05 & 128 \\
\hline 2088 & 103 & 380 & 10 & 10 & 4 & 100 & 0.08 & 0.07 & 107 & 0.07 & 0.07 & 61 \\
\hline 2090 & 203 & 760 & 10 & 20 & 2 & 100 & 4.81 & 3.67 & 740 & 4.85 & 3.72 & 544 \\
\hline 2092 & 203 & 760 & 10 & 20 & 4 & 100 & 8.52 & 7.05 & 819 & 8.45 & 7.21 & 495 \\
\hline 2094 & 303 & 1140 & 10 & 30 & 2 & 100 & 101.37 & 64.69 & 2272 & 105.14 & 63.72 & 1577 \\
\hline 2096 & 303 & 1140 & 10 & 30 & 4 & 100 & 107.88 & 75.80 & 2212 & 103.24 & 75.33 & 907 \\
\hline 2098 & 203 & 780 & 20 & 10 & 2 & 100 & 0.10 & 0.07 & 191 & 0.10 & 0.07 & 154 \\
\hline 2100 & 203 & 780 & 20 & 10 & 4 & 100 & 0.24 & 0.20 & 193 & 0.21 & 0.19 & 130 \\
\hline 2102 & 403 & 1560 & 20 & 20 & 2 & 100 & 17.22 & 11.57 & 1013 & 16.84 & 11.65 & 813 \\
\hline 2104 & 403 & 1560 & 20 & 20 & 4 & 100 & 33.92 & 31.04 & 674 & 34.13 & 30.64 & 366 \\
\hline 2106 & 603 & 2340 & 20 & 30 & 2 & 100 & 1114.05 & 645.15 & 3925 & 1057.09 & 654.00 & 2715 \\
\hline 2108 & 603 & 2340 & 20 & 30 & 4 & 100 & 1242.92 & 983.20 & 5751 & 1342.84 & 1002.42 & 2630 \\
\hline 2110 & 303 & 1180 & 30 & 10 & 2 & 100 & 0.21 & 0.14 & 149 & 0.21 & 0.15 & 126 \\
\hline 2112 & 303 & 1180 & 30 & 10 & 4 & 100 & 0.57 & 0.53 & 238 & 0.58 & 0.53 & 148 \\
\hline 2114 & 603 & 2360 & 30 & 20 & 2 & 100 & 12.58 & 10.32 & 911 & 12.87 & 10.37 & 711 \\
\hline 2116 & 603 & 2360 & 30 & 20 & 4 & 100 & 71.08 & 60.27 & 1703 & 73.71 & 61.04 & 824 \\
\hline 2118 & 903 & 3540 & 30 & 30 & 2 & 100 & 462.29 & 366.25 & 3619 & 501.12 & 366.60 & 2446 \\
\hline 2120 & 903 & 3540 & 30 & 30 & 4 & 100 & 1762.66 & 1598.68 & 4091 & 1849.92 & 1587.75 & 1823 \\
\hline
\end{tabular}

https://doi.org/10.1002/net.21815 
A.2. Extensions of Labeling Algorithms

$\stackrel{34}{\perp}$ WILEY

RAITH ET AL.

TA B L E A9 Grid instances with two objectives $(k=2)$, random scenarios and $c=10$, flimsily robust efficiency, 20 instances for each set of parameters

\begin{tabular}{|c|c|c|c|c|c|c|c|c|c|c|c|c|c|c|c|}
\hline & \multirow[b]{2}{*}{$|V|$} & \multirow[b]{2}{*}{$|E|$} & \multirow[b]{2}{*}{$\boldsymbol{h}$} & \multirow[b]{2}{*}{$w$} & \multirow[b]{2}{*}{$r$} & \multirow[b]{2}{*}{$c$} & \multicolumn{3}{|c|}{ EL-Flimsily } & \multicolumn{3}{|c|}{ RL-Flimsily } & sols & & \\
\hline & & & & & & & $\overline{\min }$ & $\max$ & $\overline{\text { avg }}$ & $\overline{\min }$ & $\max$ & $\overline{\text { avg }}$ & $\overline{\min }$ & $\max$ & $\overline{\text { avg }}$ \\
\hline 2 & 103 & 380 & 10 & 10 & 2 & 10 & 0.00 & 0.01 & 0.01 & 0.00 & 0.00 & 0.00 & 7 & 22 & 15.35 \\
\hline 4 & 103 & 380 & 10 & 10 & 4 & 10 & 0.01 & 0.03 & 0.02 & 0.00 & 0.01 & 0.00 & 23 & 43 & 30.20 \\
\hline 6 & 103 & 380 & 10 & 10 & 6 & 10 & 0.03 & 0.06 & 0.04 & 0.01 & 0.01 & 0.01 & 34 & 63 & 43.85 \\
\hline 8 & 103 & 380 & 10 & 10 & 8 & 10 & 0.06 & 0.09 & 0.08 & 0.01 & 0.01 & 0.01 & 51 & 73 & 60.60 \\
\hline 10 & 203 & 760 & 10 & 20 & 2 & 10 & 0.03 & 0.06 & 0.04 & 0.01 & 0.02 & 0.01 & 22 & 49 & 35.30 \\
\hline 12 & 203 & 760 & 10 & 20 & 4 & 10 & 0.12 & 0.24 & 0.18 & 0.02 & 0.04 & 0.03 & 52 & 81 & 66.65 \\
\hline 14 & 203 & 760 & 10 & 20 & 6 & 10 & 0.33 & 0.56 & 0.46 & 0.03 & 0.05 & 0.04 & 73 & 114 & 97.25 \\
\hline 16 & 203 & 760 & 10 & 20 & 8 & 10 & 0.77 & 1.40 & 1.01 & 0.07 & 0.14 & 0.09 & 102 & 165 & 137.85 \\
\hline 18 & 303 & 1140 & 10 & 30 & 2 & 10 & 0.07 & 0.25 & 0.15 & 0.03 & 0.12 & 0.07 & 31 & 84 & 53.35 \\
\hline 20 & 303 & 1140 & 10 & 30 & 4 & 10 & 0.54 & 1.03 & 0.79 & 0.07 & 0.17 & 0.12 & 88 & 143 & 115.70 \\
\hline 22 & 303 & 1140 & 10 & 30 & 6 & 10 & 1.42 & 3.16 & 2.19 & 0.10 & 0.24 & 0.17 & 133 & 193 & 161.20 \\
\hline 24 & 303 & 1140 & 10 & 30 & 8 & 10 & 3.26 & 5.75 & 4.67 & 0.14 & 0.25 & 0.21 & 169 & 266 & 218.65 \\
\hline 26 & 403 & 1520 & 10 & 40 & 2 & 10 & 0.25 & 0.65 & 0.43 & 0.07 & 0.18 & 0.11 & 48 & 117 & 76.35 \\
\hline 28 & 403 & 1520 & 10 & 40 & 4 & 10 & 1.49 & 3.00 & 2.20 & 0.17 & 0.32 & 0.26 & 133 & 185 & 158.55 \\
\hline 30 & 403 & 1520 & 10 & 40 & 6 & 10 & 4.39 & 9.03 & 6.83 & 0.30 & 0.54 & 0.44 & 177 & 291 & 239.75 \\
\hline 32 & 403 & 1520 & 10 & 40 & 8 & 10 & 10.87 & 20.85 & 14.88 & 0.46 & 0.79 & 0.59 & 271 & 422 & 326.85 \\
\hline 34 & 203 & 780 & 20 & 10 & 2 & 10 & 0.01 & 0.01 & 0.01 & 0.00 & 0.00 & 0.00 & 11 & 25 & 16.75 \\
\hline 36 & 203 & 780 & 20 & 10 & 4 & 10 & 0.03 & 0.05 & 0.04 & 0.01 & 0.01 & 0.01 & 25 & 45 & 32.90 \\
\hline 38 & 203 & 780 & 20 & 10 & 6 & 10 & 0.07 & 0.12 & 0.10 & 0.01 & 0.02 & 0.01 & 31 & 68 & 52.35 \\
\hline 40 & 203 & 780 & 20 & 10 & 8 & 10 & 0.16 & 0.20 & 0.17 & 0.02 & 0.02 & 0.02 & 54 & 77 & 63.65 \\
\hline 42 & 403 & 1560 & 20 & 20 & 2 & 10 & 0.06 & 0.12 & 0.09 & 0.02 & 0.04 & 0.02 & 27 & 47 & 36.95 \\
\hline 44 & 403 & 1560 & 20 & 20 & 4 & 10 & 0.32 & 0.51 & 0.40 & 0.05 & 0.07 & 0.06 & 54 & 84 & 70.15 \\
\hline 46 & 403 & 1560 & 20 & 20 & 6 & 10 & 0.76 & 1.33 & 1.09 & 0.07 & 0.11 & 0.09 & 89 & 125 & 105.10 \\
\hline 48 & 403 & 1560 & 20 & 20 & 8 & 10 & 1.81 & 2.52 & 2.21 & 0.10 & 0.14 & 0.12 & 118 & 158 & 135.30 \\
\hline 50 & 603 & 2340 & 20 & 30 & 2 & 10 & 0.26 & 0.63 & 0.38 & 0.06 & 0.17 & 0.10 & 34 & 93 & 61.50 \\
\hline 52 & 603 & 2340 & 20 & 30 & 4 & 10 & 1.25 & 2.28 & 1.77 & 0.15 & 0.27 & 0.21 & 89 & 130 & 112.45 \\
\hline 54 & 603 & 2340 & 20 & 30 & 6 & 10 & 3.97 & 6.87 & 5.14 & 0.28 & 0.46 & 0.35 & 142 & 203 & 176.25 \\
\hline 56 & 603 & 2340 & 20 & 30 & 8 & 10 & 9.88 & 12.60 & 10.88 & 0.43 & 0.55 & 0.48 & 204 & 279 & 235.55 \\
\hline 58 & 803 & 3120 & 20 & 40 & 2 & 10 & 0.58 & 1.39 & 1.05 & 0.15 & 0.40 & 0.26 & 58 & 109 & 83.90 \\
\hline 60 & 803 & 3120 & 20 & 40 & 4 & 10 & 4.14 & 8.93 & 6.08 & 0.48 & 0.94 & 0.68 & 126 & 195 & 172.10 \\
\hline 62 & 803 & 3120 & 20 & 40 & 6 & 10 & 12.39 & 24.54 & 17.70 & 0.77 & 1.41 & 1.09 & 206 & 310 & 254.70 \\
\hline 64 & 803 & 3120 & 20 & 40 & 8 & 10 & 27.27 & 46.56 & 38.35 & 1.06 & 1.71 & 1.44 & 277 & 388 & 334.90 \\
\hline 66 & 303 & 1180 & 30 & 10 & 2 & 10 & 0.01 & 0.02 & 0.02 & 0.00 & 0.01 & 0.01 & 11 & 26 & 17.40 \\
\hline 68 & 303 & 1180 & 30 & 10 & 4 & 10 & 0.05 & 0.07 & 0.06 & 0.01 & 0.01 & 0.01 & 28 & 46 & 35.85 \\
\hline 70 & 303 & 1180 & 30 & 10 & 6 & 10 & 0.13 & 0.17 & 0.15 & 0.02 & 0.02 & 0.02 & 42 & 64 & 54.90 \\
\hline 72 & 303 & 1180 & 30 & 10 & 8 & 10 & 0.27 & 0.33 & 0.29 & 0.03 & 0.03 & 0.03 & 56 & 82 & 69.05 \\
\hline 74 & 603 & 2360 & 30 & 20 & 2 & 10 & 0.10 & 0.18 & 0.13 & 0.03 & 0.05 & 0.04 & 26 & 61 & 35.60 \\
\hline 76 & 603 & 2360 & 30 & 20 & 4 & 10 & 0.50 & 0.76 & 0.64 & 0.07 & 0.11 & 0.09 & 57 & 100 & 75.55 \\
\hline 78 & 603 & 2360 & 30 & 20 & 6 & 10 & 1.48 & 1.97 & 1.68 & 0.12 & 0.16 & 0.14 & 93 & 131 & 111.15 \\
\hline 80 & 603 & 2360 & 30 & 20 & 8 & 10 & 3.07 & 4.18 & 3.51 & 0.17 & 0.22 & 0.19 & 122 & 168 & 143.85 \\
\hline 82 & 903 & 3540 & 30 & 30 & 2 & 10 & 0.40 & 0.79 & 0.60 & 0.11 & 0.20 & 0.15 & 45 & 71 & 56.90 \\
\hline 84 & 903 & 3540 & 30 & 30 & 4 & 10 & 2.12 & 3.52 & 2.90 & 0.25 & 0.42 & 0.34 & 96 & 154 & 117.90 \\
\hline 86 & 903 & 3540 & 30 & 30 & 6 & 10 & 6.59 & 10.13 & 8.42 & 0.45 & 0.68 & 0.57 & 133 & 216 & 177.50 \\
\hline 88 & 903 & 3540 & 30 & 30 & 8 & 10 & 15.69 & 20.75 & 18.72 & 0.68 & 0.88 & 0.80 & 203 & 275 & 238.55 \\
\hline 90 & 1203 & 4720 & 30 & 40 & 2 & 10 & 1.24 & 2.31 & 1.83 & 0.30 & 0.54 & 0.43 & 68 & 101 & 89.35 \\
\hline 92 & 1203 & 4720 & 30 & 40 & 4 & 10 & 7.62 & 10.91 & 9.04 & 0.81 & 1.22 & 1.02 & 129 & 220 & 173.40 \\
\hline 94 & 1203 & 4720 & 30 & 40 & 6 & 10 & 20.49 & 34.46 & 27.34 & 1.34 & 2.09 & 1.70 & 225 & 327 & 263.75 \\
\hline 96 & 1203 & 4720 & 30 & 40 & 8 & 10 & 50.04 & 71.68 & 59.48 & 1.97 & 2.78 & 2.31 & 289 & 382 & 344.40 \\
\hline 98 & 403 & 1580 & 40 & 10 & 2 & 10 & 0.02 & 0.03 & 0.02 & 0.01 & 0.01 & 0.01 & 10 & 30 & 18.80 \\
\hline 100 & 403 & 1580 & 40 & 10 & 4 & 10 & 0.07 & 0.10 & 0.09 & 0.01 & 0.02 & 0.02 & 26 & 45 & 36.30 \\
\hline 102 & 403 & 1580 & 40 & 10 & 6 & 10 & 0.18 & 0.23 & 0.21 & 0.02 & 0.03 & 0.03 & 41 & 71 & 55.75 \\
\hline 104 & 403 & 1580 & 40 & 10 & 8 & 10 & 0.36 & 0.46 & 0.40 & 0.04 & 0.04 & 0.04 & 58 & 86 & 71.25 \\
\hline 106 & 803 & 3160 & 40 & 20 & 2 & 10 & 0.15 & 0.24 & 0.20 & 0.04 & 0.07 & 0.05 & 27 & 45 & 36.45 \\
\hline 108 & 803 & 3160 & 40 & 20 & 4 & 10 & 0.70 & 1.04 & 0.84 & 0.10 & 0.14 & 0.12 & 47 & 89 & 72.30 \\
\hline 110 & 803 & 3160 & 40 & 20 & 6 & 10 & 2.00 & 2.67 & 2.35 & 0.17 & 0.21 & 0.19 & 73 & 144 & 111.60 \\
\hline 112 & 803 & 3160 & 40 & 20 & 8 & 10 & 4.03 & 5.74 & 4.90 & 0.23 & 0.30 & 0.26 & 127 & 192 & 147.15 \\
\hline 114 & 1203 & 4740 & 40 & 30 & 2 & 10 & 0.56 & 1.03 & 0.75 & 0.14 & 0.25 & 0.19 & 39 & 79 & 59.70 \\
\hline 116 & 1203 & 4740 & 40 & 30 & 4 & 10 & 3.17 & 4.69 & 3.93 & 0.39 & 0.56 & 0.48 & 94 & 141 & 119.75 \\
\hline 118 & 1203 & 4740 & 40 & 30 & 6 & 10 & 9.97 & 15.74 & 11.34 & 0.71 & 1.01 & 0.79 & 148 & 233 & 188.85 \\
\hline 120 & 1203 & 4740 & 40 & 30 & 8 & 10 & 20.71 & 27.80 & 23.90 & 0.94 & 1.17 & 1.06 & 188 & 270 & 226.15 \\
\hline 122 & 1603 & 6320 & 40 & 40 & 2 & 10 & 1.77 & 3.21 & 2.34 & 0.40 & 0.76 & 0.58 & 50 & 118 & 89.05 \\
\hline 124 & 1603 & 6320 & 40 & 40 & 4 & 10 & 9.30 & 18.57 & 12.46 & 1.12 & 2.22 & 1.46 & 157 & 232 & 187.40 \\
\hline 126 & 1603 & 6320 & 40 & 40 & 6 & 10 & 29.73 & 43.57 & 37.70 & 1.98 & 2.69 & 2.36 & 215 & 305 & 273.80 \\
\hline 128 & 1603 & 6320 & 40 & 40 & 8 & 10 & 79.32 & 96.18 & 85.94 & 3.07 & 3.87 & 3.38 & 296 & 449 & 352.50 \\
\hline
\end{tabular}

https://doi.org/10.1002/net.21815 
RAITH ET AL.

WILEY

T A B LE A10 Grid instances with two objectives $(k=2)$, random scenarios and $c=100$, flimsily robust efficiency, 20 instances for each set of parameters

\begin{tabular}{|c|c|c|c|c|c|c|c|c|c|c|c|c|c|c|c|}
\hline \multirow{2}{*}{\multicolumn{2}{|c|}{$|V|$}} & \multirow[b]{2}{*}{$|E|$} & \multirow[b]{2}{*}{$\boldsymbol{h}$} & \multirow[b]{2}{*}{$w$} & \multirow[b]{2}{*}{$r$} & \multirow[b]{2}{*}{$c$} & \multicolumn{3}{|c|}{ EL-Flimsily } & \multicolumn{3}{|c|}{ RL-Flimsily } & sols & & \\
\hline & & & & & & & $\overline{\min }$ & $\max$ & $\overline{\text { avg }}$ & $\overline{\min }$ & $\max$ & $\overline{\text { avg }}$ & $\overline{\min }$ & $\max$ & $\overline{\text { avg }}$ \\
\hline 130 & 103 & 380 & 10 & 10 & 2 & 100 & 0.01 & 0.01 & 0.01 & 0.00 & 0.01 & 0.00 & 11 & 32 & 23.25 \\
\hline 132 & 103 & 380 & 10 & 10 & 4 & 100 & 0.02 & 0.05 & 0.04 & 0.00 & 0.01 & 0.01 & 24 & 60 & 41.20 \\
\hline 134 & 103 & 380 & 10 & 10 & 6 & 100 & 0.07 & 0.13 & 0.08 & 0.01 & 0.02 & 0.01 & 50 & 102 & 66.55 \\
\hline 136 & 103 & 380 & 10 & 10 & 8 & 100 & 0.13 & 0.22 & 0.16 & 0.01 & 0.03 & 0.01 & 69 & 111 & 87.75 \\
\hline 138 & 203 & 760 & 10 & 20 & 2 & 100 & 0.04 & 0.14 & 0.07 & 0.01 & 0.04 & 0.02 & 30 & 74 & 48.90 \\
\hline 140 & 203 & 760 & 10 & 20 & 4 & 100 & 0.30 & 0.62 & 0.41 & 0.04 & 0.08 & 0.06 & 72 & 154 & 101.85 \\
\hline 142 & 203 & 760 & 10 & 20 & 6 & 100 & 0.77 & 1.39 & 1.07 & 0.06 & 0.12 & 0.09 & 130 & 206 & 160.65 \\
\hline 144 & 203 & 760 & 10 & 20 & 8 & 100 & 1.51 & 3.27 & 2.22 & 0.08 & 0.17 & 0.11 & 160 & 237 & 195.35 \\
\hline 146 & 303 & 1140 & 10 & 30 & 2 & 100 & 0.24 & 0.69 & 0.36 & 0.06 & 0.18 & 0.09 & 56 & 135 & 87.95 \\
\hline 148 & 303 & 1140 & 10 & 30 & 4 & 100 & 1.40 & 2.71 & 2.00 & 0.16 & 0.34 & 0.24 & 138 & 235 & 184.55 \\
\hline 150 & 303 & 1140 & 10 & 30 & 6 & 100 & 4.25 & 7.88 & 5.61 & 0.29 & 0.53 & 0.36 & 233 & 311 & 267.15 \\
\hline 152 & 303 & 1140 & 10 & 30 & 8 & 100 & 10.24 & 20.88 & 13.38 & 0.41 & 0.82 & 0.53 & 308 & 464 & 372.70 \\
\hline 154 & 403 & 1520 & 10 & 40 & 2 & 100 & 0.48 & 1.87 & 1.32 & 0.15 & 0.49 & 0.34 & 91 & 199 & 143.85 \\
\hline 156 & 403 & 1520 & 10 & 40 & 4 & 100 & 3.78 & 9.37 & 6.19 & 0.49 & 1.30 & 0.82 & 192 & 321 & 268.25 \\
\hline 158 & 403 & 1520 & 10 & 40 & 6 & 100 & 13.52 & 37.13 & 21.72 & 0.77 & 2.86 & 1.23 & 337 & 532 & 407.90 \\
\hline 160 & 403 & 1520 & 10 & 40 & 8 & 100 & 30.67 & 64.74 & 46.31 & 1.09 & 1.98 & 1.48 & 429 & 674 & 554.40 \\
\hline 162 & 203 & 780 & 20 & 10 & 2 & 100 & 0.01 & 0.04 & 0.02 & 0.00 & 0.01 & 0.01 & 17 & 41 & 26.60 \\
\hline 164 & 203 & 780 & 20 & 10 & 4 & 100 & 0.07 & 0.12 & 0.08 & 0.01 & 0.02 & 0.02 & 39 & 66 & 50.45 \\
\hline 166 & 203 & 780 & 20 & 10 & 6 & 100 & 0.16 & 0.24 & 0.20 & 0.02 & 0.03 & 0.02 & 59 & 94 & 74.40 \\
\hline 168 & 203 & 780 & 20 & 10 & 8 & 100 & 0.32 & 0.51 & 0.39 & 0.03 & 0.05 & 0.03 & 78 & 122 & 98.55 \\
\hline 170 & 403 & 1560 & 20 & 20 & 2 & 100 & 0.12 & 0.37 & 0.21 & 0.04 & 0.09 & 0.06 & 39 & 90 & 59.25 \\
\hline 172 & 403 & 1560 & 20 & 20 & 4 & 100 & 0.67 & 1.28 & 0.94 & 0.09 & 0.16 & 0.12 & 82 & 132 & 106.95 \\
\hline 174 & 403 & 1560 & 20 & 20 & 6 & 100 & 1.79 & 3.16 & 2.52 & 0.16 & 0.25 & 0.20 & 143 & 200 & 168.75 \\
\hline 176 & 403 & 1560 & 20 & 20 & 8 & 100 & 4.37 & 7.26 & 5.51 & 0.23 & 0.35 & 0.27 & 170 & 257 & 221.55 \\
\hline 178 & 603 & 2340 & 20 & 30 & 2 & 100 & 0.51 & 1.23 & 0.84 & 0.14 & 0.30 & 0.22 & 66 & 118 & 92.90 \\
\hline 180 & 603 & 2340 & 20 & 30 & 4 & 100 & 3.31 & 6.54 & 4.84 & 0.39 & 0.68 & 0.54 & 161 & 237 & 194.90 \\
\hline 182 & 603 & 2340 & 20 & 30 & 6 & 100 & 11.78 & 18.09 & 13.88 & 0.67 & 1.04 & 0.83 & 234 & 356 & 280.90 \\
\hline 184 & 603 & 2340 & 20 & 30 & 8 & 100 & 23.98 & 38.39 & 32.07 & 0.93 & 1.57 & 1.17 & 334 & 441 & 380.50 \\
\hline 186 & 803 & 3120 & 20 & 40 & 2 & 100 & 2.24 & 4.33 & 2.93 & 0.46 & 1.06 & 0.76 & 113 & 203 & 142.55 \\
\hline 188 & 803 & 3120 & 20 & 40 & 4 & 100 & 11.19 & 20.95 & 16.57 & 1.29 & 2.42 & 1.88 & 239 & 379 & 297.10 \\
\hline 190 & 803 & 3120 & 20 & 40 & 6 & 100 & 41.37 & 73.48 & 55.11 & 2.16 & 3.75 & 2.94 & 343 & 530 & 443.30 \\
\hline 192 & 803 & 3120 & 20 & 40 & 8 & 100 & 94.52 & 157.72 & 129.16 & 2.87 & 6.05 & 4.26 & 507 & 697 & 601.15 \\
\hline 194 & 303 & 1180 & 30 & 10 & 2 & 100 & 0.03 & 0.05 & 0.03 & 0.01 & 0.02 & 0.01 & 19 & 36 & 27.35 \\
\hline 196 & 303 & 1180 & 30 & 10 & 4 & 100 & 0.10 & 0.15 & 0.12 & 0.02 & 0.02 & 0.02 & 41 & 57 & 48.60 \\
\hline 198 & 303 & 1180 & 30 & 10 & 6 & 100 & 0.29 & 0.39 & 0.33 & 0.03 & 0.05 & 0.04 & 60 & 100 & 75.05 \\
\hline 200 & 303 & 1180 & 30 & 10 & 8 & 100 & 0.53 & 0.75 & 0.61 & 0.04 & 0.06 & 0.05 & 82 & 120 & 98.55 \\
\hline 202 & 603 & 2360 & 30 & 20 & 2 & 100 & 0.23 & 0.43 & 0.33 & 0.06 & 0.11 & 0.09 & 38 & 75 & 58.45 \\
\hline 204 & 603 & 2360 & 30 & 20 & 4 & 100 & 1.10 & 1.94 & 1.53 & 0.14 & 0.27 & 0.20 & 88 & 148 & 118.80 \\
\hline 206 & 603 & 2360 & 30 & 20 & 6 & 100 & 3.29 & 5.44 & 4.27 & 0.25 & 0.39 & 0.30 & 127 & 253 & 175.45 \\
\hline 208 & 603 & 2360 & 30 & 20 & 8 & 100 & 7.83 & 10.43 & 9.17 & 0.36 & 0.47 & 0.42 & 181 & 283 & 231.85 \\
\hline 210 & 903 & 3540 & 30 & 30 & 2 & 100 & 0.90 & 1.95 & 1.44 & 0.23 & 0.50 & 0.37 & 68 & 158 & 96.70 \\
\hline 212 & 903 & 3540 & 30 & 30 & 4 & 100 & 5.77 & 8.79 & 7.06 & 0.66 & 0.99 & 0.80 & 158 & 241 & 197.00 \\
\hline 214 & 903 & 3540 & 30 & 30 & 6 & 100 & 16.89 & 28.39 & 23.41 & 1.03 & 1.62 & 1.36 & 231 & 326 & 279.30 \\
\hline 216 & 903 & 3540 & 30 & 30 & 8 & 100 & 44.50 & 63.35 & 52.60 & 1.67 & 2.35 & 1.99 & 338 & 493 & 387.95 \\
\hline 218 & 1203 & 4720 & 30 & 40 & 2 & 100 & 3.56 & 6.65 & 4.88 & 0.81 & 1.91 & 1.25 & 111 & 189 & 151.95 \\
\hline 220 & 1203 & 4720 & 30 & 40 & 4 & 100 & 21.36 & 32.03 & 25.69 & 2.39 & 3.65 & 2.84 & 219 & 322 & 287.50 \\
\hline 222 & 1203 & 4720 & 30 & 40 & 6 & 100 & 74.43 & 110.14 & 86.56 & 4.09 & 6.55 & 5.23 & 369 & 536 & 442.05 \\
\hline 224 & 1203 & 4720 & 30 & 40 & 8 & 100 & 166.00 & 256.82 & 209.89 & 5.63 & 8.72 & 7.06 & 474 & 719 & 598.85 \\
\hline 226 & 403 & 1580 & 40 & 10 & 2 & 100 & 0.03 & 0.06 & 0.04 & 0.01 & 0.02 & 0.01 & 15 & 46 & 26.90 \\
\hline 228 & 403 & 1580 & 40 & 10 & 4 & 100 & 0.14 & 0.23 & 0.17 & 0.02 & 0.06 & 0.03 & 35 & 75 & 52.35 \\
\hline 230 & 403 & 1580 & 40 & 10 & 6 & 100 & 0.36 & 0.50 & 0.43 & 0.04 & 0.07 & 0.05 & 55 & 97 & 76.10 \\
\hline 232 & 403 & 1580 & 40 & 10 & 8 & 100 & 0.72 & 1.00 & 0.82 & 0.06 & 0.08 & 0.07 & 90 & 144 & 106.05 \\
\hline 234 & 803 & 3160 & 40 & 20 & 2 & 100 & 0.27 & 0.48 & 0.40 & 0.07 & 0.16 & 0.12 & 33 & 83 & 57.90 \\
\hline 236 & 803 & 3160 & 40 & 20 & 4 & 100 & 1.53 & 2.27 & 1.96 & 0.20 & 0.38 & 0.27 & 83 & 134 & 110.65 \\
\hline 238 & 803 & 3160 & 40 & 20 & 6 & 100 & 4.64 & 6.75 & 5.61 & 0.34 & 0.54 & 0.42 & 147 & 205 & 174.25 \\
\hline 240 & 803 & 3160 & 40 & 20 & 8 & 100 & 10.61 & 17.16 & 12.84 & 0.51 & 0.83 & 0.60 & 194 & 286 & 230.80 \\
\hline 242 & 1203 & 4740 & 40 & 30 & 2 & 100 & 1.44 & 2.36 & 1.92 & 0.38 & 0.65 & 0.51 & 65 & 120 & 95.00 \\
\hline 244 & 1203 & 4740 & 40 & 30 & 4 & 100 & 7.86 & 13.25 & 10.57 & 0.97 & 1.63 & 1.30 & 162 & 230 & 199.80 \\
\hline 246 & 1203 & 4740 & 40 & 30 & 6 & 100 & 23.77 & 39.15 & 31.23 & 1.49 & 2.31 & 1.90 & 228 & 352 & 291.10 \\
\hline 248 & 1203 & 4740 & 40 & 30 & 8 & 100 & 65.11 & 82.88 & 73.53 & 2.40 & 3.18 & 2.77 & 343 & 489 & 409.90 \\
\hline 250 & 1603 & 6320 & 40 & 40 & 2 & 100 & 4.44 & 8.98 & 6.15 & 1.13 & 2.40 & 1.72 & 103 & 217 & 146.65 \\
\hline 252 & 1603 & 6320 & 40 & 40 & 4 & 100 & 27.39 & 43.30 & 35.39 & 3.01 & 6.18 & 4.37 & 225 & 387 & 299.90 \\
\hline 254 & 1603 & 6320 & 40 & 40 & 6 & 100 & 94.92 & 139.25 & 118.61 & 5.47 & 10.46 & 6.83 & 345 & 500 & 445.05 \\
\hline 256 & 1603 & 6320 & 40 & 40 & 8 & 100 & 236.51 & 336.08 & 276.11 & 7.87 & 12.33 & 9.56 & 511 & 685 & 592.40 \\
\hline
\end{tabular}

https://doi.org/10.1002/net.21815 
A.2. Extensions of Labeling Algorithms

${ }^{36}-$ WI LEY

RAITH ET AL.

TA B LE A11 Grid instances with two objectives $(k=2)$, random scenarios and $c=10$, highly robust efficiency, 20 instances for each set of parameters

\begin{tabular}{|c|c|c|c|c|c|c|c|c|c|c|c|c|c|c|c|}
\hline \multirow{2}{*}{\multicolumn{2}{|c|}{$|V|$}} & \multirow[b]{2}{*}{$|E|$} & \multirow[b]{2}{*}{$\boldsymbol{h}$} & \multirow[b]{2}{*}{$w$} & \multirow[b]{2}{*}{$r$} & \multirow[b]{2}{*}{$c$} & \multicolumn{3}{|c|}{ EL-Highly } & \multicolumn{3}{|c|}{ RL-Highly } & sols & & \\
\hline & & & & & & & $\overline{\min }$ & $\max$ & $\overline{\text { avg }}$ & $\overline{\min }$ & $\max$ & $\overline{\text { avg }}$ & $\overline{\min }$ & $\max$ & $\overline{\text { avg }}$ \\
\hline 2 & 103 & 380 & 10 & 10 & 2 & 10 & 0.00 & 0.01 & 0.01 & 0.00 & 0.00 & 0.00 & 0 & 1 & 0.05 \\
\hline 4 & 103 & 380 & 10 & 10 & 4 & 10 & 0.01 & 0.03 & 0.02 & 0.00 & 0.01 & 0.00 & 0 & 0 & 0.00 \\
\hline 6 & 103 & 380 & 10 & 10 & 6 & 10 & 0.03 & 0.06 & 0.04 & 0.00 & 0.01 & 0.01 & 0 & 0 & 0.00 \\
\hline 8 & 103 & 380 & 10 & 10 & 8 & 10 & 0.06 & 0.10 & 0.08 & 0.01 & 0.01 & 0.01 & 0 & 0 & 0.00 \\
\hline 10 & 203 & 760 & 10 & 20 & 2 & 10 & 0.03 & 0.06 & 0.04 & 0.01 & 0.02 & 0.01 & 0 & 1 & 0.05 \\
\hline 12 & 203 & 760 & 10 & 20 & 4 & 10 & 0.12 & 0.25 & 0.18 & 0.02 & 0.03 & 0.03 & 0 & 0 & 0.00 \\
\hline 14 & 203 & 760 & 10 & 20 & 6 & 10 & 0.34 & 0.56 & 0.46 & 0.03 & 0.05 & 0.04 & 0 & 0 & 0.00 \\
\hline 16 & 203 & 760 & 10 & 20 & 8 & 10 & 0.80 & 1.37 & 1.02 & 0.05 & 0.07 & 0.06 & 0 & 0 & 0.00 \\
\hline 18 & 303 & 1140 & 10 & 30 & 2 & 10 & 0.07 & 0.26 & 0.15 & 0.02 & 0.07 & 0.04 & 0 & 0 & 0.00 \\
\hline 20 & 303 & 1140 & 10 & 30 & 4 & 10 & 0.55 & 1.02 & 0.80 & 0.07 & 0.13 & 0.10 & 0 & 0 & 0.00 \\
\hline 22 & 303 & 1140 & 10 & 30 & 6 & 10 & 1.44 & 3.26 & 2.24 & 0.10 & 0.21 & 0.16 & 0 & 0 & 0.00 \\
\hline 24 & 303 & 1140 & 10 & 30 & 8 & 10 & 3.04 & 5.91 & 4.70 & 0.14 & 0.24 & 0.20 & 0 & 0 & 0.00 \\
\hline 26 & 403 & 1520 & 10 & 40 & 2 & 10 & 0.26 & 0.66 & 0.43 & 0.07 & 0.18 & 0.11 & 0 & 0 & 0.00 \\
\hline 28 & 403 & 1520 & 10 & 40 & 4 & 10 & 1.51 & 2.95 & 2.25 & 0.17 & 0.31 & 0.25 & 0 & 0 & 0.00 \\
\hline 30 & 403 & 1520 & 10 & 40 & 6 & 10 & 4.47 & 9.36 & 7.05 & 0.29 & 0.53 & 0.43 & 0 & 0 & 0.00 \\
\hline 32 & 403 & 1520 & 10 & 40 & 8 & 10 & 10.91 & 21.87 & 15.39 & 0.45 & 0.79 & 0.58 & 0 & 0 & 0.00 \\
\hline 34 & 203 & 780 & 20 & 10 & 2 & 10 & 0.01 & 0.01 & 0.01 & 0.00 & 0.00 & 0.00 & 0 & 1 & 0.05 \\
\hline 36 & 203 & 780 & 20 & 10 & 4 & 10 & 0.03 & 0.05 & 0.04 & 0.01 & 0.01 & 0.01 & 0 & 0 & 0.00 \\
\hline 38 & 203 & 780 & 20 & 10 & 6 & 10 & 0.07 & 0.12 & 0.10 & 0.01 & 0.02 & 0.01 & 0 & 0 & 0.00 \\
\hline 40 & 203 & 780 & 20 & 10 & 8 & 10 & 0.16 & 0.20 & 0.18 & 0.02 & 0.02 & 0.02 & 0 & 0 & 0.00 \\
\hline 42 & 403 & 1560 & 20 & 20 & 2 & 10 & 0.06 & 0.13 & 0.09 & 0.02 & 0.04 & 0.02 & 0 & 0 & 0.00 \\
\hline 44 & 403 & 1560 & 20 & 20 & 4 & 10 & 0.32 & 0.51 & 0.40 & 0.05 & 0.07 & 0.06 & 0 & 0 & 0.00 \\
\hline 46 & 403 & 1560 & 20 & 20 & 6 & 10 & 0.81 & 1.35 & 1.11 & 0.07 & 0.11 & 0.09 & 0 & 0 & 0.00 \\
\hline 48 & 403 & 1560 & 20 & 20 & 8 & 10 & 1.83 & 2.69 & 2.27 & 0.10 & 0.14 & 0.12 & 0 & 0 & 0.00 \\
\hline 50 & 603 & 2340 & 20 & 30 & 2 & 10 & 0.26 & 0.64 & 0.38 & 0.06 & 0.17 & 0.10 & 0 & 0 & 0.00 \\
\hline 52 & 603 & 2340 & 20 & 30 & 4 & 10 & 1.34 & 2.28 & 1.82 & 0.15 & 0.27 & 0.21 & 0 & 0 & 0.00 \\
\hline 54 & 603 & 2340 & 20 & 30 & 6 & 10 & 4.06 & 7.04 & 5.29 & 0.28 & 0.46 & 0.35 & 0 & 0 & 0.00 \\
\hline 56 & 603 & 2340 & 20 & 30 & 8 & 10 & 9.96 & 12.92 & 11.13 & 0.43 & 0.55 & 0.48 & 0 & 0 & 0.00 \\
\hline 58 & 803 & 3120 & 20 & 40 & 2 & 10 & 0.60 & 1.44 & 1.09 & 0.14 & 0.41 & 0.26 & 0 & 0 & 0.00 \\
\hline 60 & 803 & 3120 & 20 & 40 & 4 & 10 & 4.29 & 9.33 & 6.27 & 0.47 & 0.95 & 0.68 & 0 & 0 & 0.00 \\
\hline 62 & 803 & 3120 & 20 & 40 & 6 & 10 & 11.96 & 24.82 & 17.89 & 0.77 & 1.40 & 1.07 & 0 & 0 & 0.00 \\
\hline 64 & 803 & 3120 & 20 & 40 & 8 & 10 & 28.02 & 47.04 & 38.95 & 1.06 & 1.69 & 1.43 & 0 & 0 & 0.00 \\
\hline 66 & 303 & 1180 & 30 & 10 & 2 & 10 & 0.01 & 0.02 & 0.02 & 0.00 & 0.01 & 0.01 & 0 & 0 & 0.00 \\
\hline 68 & 303 & 1180 & 30 & 10 & 4 & 10 & 0.06 & 0.08 & 0.07 & 0.01 & 0.01 & 0.01 & 0 & 0 & 0.00 \\
\hline 70 & 303 & 1180 & 30 & 10 & 6 & 10 & 0.13 & 0.17 & 0.15 & 0.02 & 0.02 & 0.02 & 0 & 0 & 0.00 \\
\hline 72 & 303 & 1180 & 30 & 10 & 8 & 10 & 0.27 & 0.33 & 0.29 & 0.03 & 0.03 & 0.03 & 0 & 0 & 0.00 \\
\hline 74 & 603 & 2360 & 30 & 20 & 2 & 10 & 0.10 & 0.18 & 0.13 & 0.03 & 0.05 & 0.04 & 0 & 0 & 0.00 \\
\hline 76 & 603 & 2360 & 30 & 20 & 4 & 10 & 0.50 & 0.76 & 0.65 & 0.07 & 0.10 & 0.09 & 0 & 0 & 0.00 \\
\hline 78 & 603 & 2360 & 30 & 20 & 6 & 10 & 1.50 & 2.15 & 1.72 & 0.12 & 0.16 & 0.14 & 0 & 0 & 0.00 \\
\hline 80 & 603 & 2360 & 30 & 20 & 8 & 10 & 3.16 & 4.31 & 3.58 & 0.17 & 0.22 & 0.19 & 0 & 0 & 0.00 \\
\hline 82 & 903 & 3540 & 30 & 30 & 2 & 10 & 0.40 & 0.79 & 0.61 & 0.11 & 0.20 & 0.15 & 0 & 0 & 0.00 \\
\hline 84 & 903 & 3540 & 30 & 30 & 4 & 10 & 2.18 & 3.45 & 2.94 & 0.25 & 0.41 & 0.34 & 0 & 0 & 0.00 \\
\hline 86 & 903 & 3540 & 30 & 30 & 6 & 10 & 6.56 & 10.16 & 8.49 & 0.44 & 0.70 & 0.57 & 0 & 0 & 0.00 \\
\hline 88 & 903 & 3540 & 30 & 30 & 8 & 10 & 15.88 & 21.04 & 18.99 & 0.68 & 1.01 & 0.81 & 0 & 0 & 0.00 \\
\hline 90 & 1203 & 4720 & 30 & 40 & 2 & 10 & 1.23 & 2.29 & 1.85 & 0.30 & 0.53 & 0.44 & 0 & 0 & 0.00 \\
\hline 92 & 1203 & 4720 & 30 & 40 & 4 & 10 & 7.60 & 10.96 & 9.08 & 0.80 & 1.19 & 1.02 & 0 & 0 & 0.00 \\
\hline 94 & 1203 & 4720 & 30 & 40 & 6 & 10 & 20.80 & 35.20 & 27.99 & 1.32 & 2.09 & 1.69 & 0 & 0 & 0.00 \\
\hline 96 & 1203 & 4720 & 30 & 40 & 8 & 10 & 51.11 & 72.96 & 60.48 & 2.01 & 2.77 & 2.30 & 0 & 0 & 0.00 \\
\hline 98 & 403 & 1580 & 40 & 10 & 2 & 10 & 0.02 & 0.03 & 0.02 & 0.01 & 0.01 & 0.01 & 0 & 1 & 0.15 \\
\hline 100 & 403 & 1580 & 40 & 10 & 4 & 10 & 0.07 & 0.10 & 0.09 & 0.01 & 0.02 & 0.02 & 0 & 0 & 0.00 \\
\hline 102 & 403 & 1580 & 40 & 10 & 6 & 10 & 0.18 & 0.24 & 0.21 & 0.02 & 0.03 & 0.03 & 0 & 0 & 0.00 \\
\hline 104 & 403 & 1580 & 40 & 10 & 8 & 10 & 0.36 & 0.51 & 0.41 & 0.03 & 0.04 & 0.04 & 0 & 0 & 0.00 \\
\hline 106 & 803 & 3160 & 40 & 20 & 2 & 10 & 0.15 & 0.24 & 0.20 & 0.04 & 0.07 & 0.05 & 0 & 0 & 0.00 \\
\hline 108 & 803 & 3160 & 40 & 20 & 4 & 10 & 0.73 & 1.08 & 0.86 & 0.10 & 0.14 & 0.12 & 0 & 0 & 0.00 \\
\hline 110 & 803 & 3160 & 40 & 20 & 6 & 10 & 2.02 & 2.77 & 2.39 & 0.17 & 0.21 & 0.19 & 0 & 0 & 0.00 \\
\hline 112 & 803 & 3160 & 40 & 20 & 8 & 10 & 4.11 & 5.83 & 5.06 & 0.23 & 0.29 & 0.26 & 0 & 0 & 0.00 \\
\hline 114 & 1203 & 4740 & 40 & 30 & 2 & 10 & 0.58 & 1.04 & 0.76 & 0.14 & 0.25 & 0.19 & 0 & 0 & 0.00 \\
\hline 116 & 1203 & 4740 & 40 & 30 & 4 & 10 & 3.26 & 4.79 & 3.98 & 0.40 & 0.56 & 0.48 & 0 & 0 & 0.00 \\
\hline 118 & 1203 & 4740 & 40 & 30 & 6 & 10 & 10.24 & 16.01 & 11.52 & 0.71 & 0.99 & 0.79 & 0 & 0 & 0.00 \\
\hline 120 & 1203 & 4740 & 40 & 30 & 8 & 10 & 21.25 & 28.06 & 24.39 & 0.91 & 1.14 & 1.05 & 0 & 0 & 0.00 \\
\hline 122 & 1603 & 6320 & 40 & 40 & 2 & 10 & 1.73 & 3.46 & 2.36 & 0.40 & 0.75 & 0.58 & 0 & 0 & 0.00 \\
\hline 124 & 1603 & 6320 & 40 & 40 & 4 & 10 & 9.44 & 18.74 & 12.66 & 1.11 & 2.22 & 1.46 & 0 & 0 & 0.00 \\
\hline 126 & 1603 & 6320 & 40 & 40 & 6 & 10 & 30.90 & 44.19 & 38.33 & 2.13 & 2.73 & 2.38 & 0 & 0 & 0.00 \\
\hline 128 & 1603 & 6320 & 40 & 40 & 8 & 10 & 80.07 & 97.28 & 87.13 & 2.94 & 3.77 & 3.34 & 0 & 0 & 0.00 \\
\hline
\end{tabular}

https://doi.org/10.1002/net.21815 
RAITH ET AL.

WILEY

T A B LE A12 Grid instances with two objectives $(k=2)$, random scenarios and $c=100$, highly robust efficiency, 20 instances for each set of parameters

\begin{tabular}{|c|c|c|c|c|c|c|c|c|c|c|c|c|c|c|c|}
\hline & \multirow[b]{2}{*}{$|V|$} & \multirow[b]{2}{*}{$|E|$} & \multirow[b]{2}{*}{$h$} & \multirow[b]{2}{*}{$w$} & \multirow[b]{2}{*}{$r$} & \multirow[b]{2}{*}{$c$} & \multicolumn{3}{|c|}{ EL-Highly } & \multicolumn{3}{|c|}{ RL-Highly } & sols & & \\
\hline & & & & & & & $\overline{\min }$ & $\max$ & $\overline{\text { avg }}$ & $\overline{\min }$ & $\max$ & $\overline{\text { avg }}$ & $\overline{\min }$ & $\max$ & avg \\
\hline 130 & 103 & 380 & 10 & 10 & 2 & 100 & 0.01 & 0.02 & 0.01 & 0.00 & 0.01 & 0.00 & 0 & 1 & 0.05 \\
\hline 132 & 103 & 380 & 10 & 10 & 4 & 100 & 0.02 & 0.06 & 0.04 & 0.00 & 0.01 & 0.01 & 0 & 0 & 0.00 \\
\hline 134 & 103 & 380 & 10 & 10 & 6 & 100 & 0.06 & 0.13 & 0.08 & 0.01 & 0.02 & 0.01 & 0 & 0 & 0.00 \\
\hline 136 & 103 & 380 & 10 & 10 & 8 & 100 & 0.13 & 0.23 & 0.17 & 0.01 & 0.02 & 0.01 & 0 & 0 & 0.00 \\
\hline 138 & 203 & 760 & 10 & 20 & 2 & 100 & 0.04 & 0.15 & 0.07 & 0.01 & 0.05 & 0.02 & 0 & 1 & 0.05 \\
\hline 140 & 203 & 760 & 10 & 20 & 4 & 100 & 0.30 & 0.62 & 0.41 & 0.04 & 0.08 & 0.06 & 0 & 0 & 0.00 \\
\hline 142 & 203 & 760 & 10 & 20 & 6 & 100 & 0.77 & 1.46 & 1.08 & 0.06 & 0.11 & 0.08 & 0 & 0 & 0.00 \\
\hline 144 & 203 & 760 & 10 & 20 & 8 & 100 & 1.51 & 3.28 & 2.23 & 0.08 & 0.18 & 0.11 & 0 & 0 & 0.00 \\
\hline 146 & 303 & 1140 & 10 & 30 & 2 & 100 & 0.25 & 0.72 & 0.37 & 0.06 & 0.17 & 0.10 & 0 & 0 & 0.00 \\
\hline 148 & 303 & 1140 & 10 & 30 & 4 & 100 & 1.38 & 2.71 & 2.00 & 0.17 & 0.32 & 0.25 & 0 & 0 & 0.00 \\
\hline 150 & 303 & 1140 & 10 & 30 & 6 & 100 & 4.23 & 8.16 & 5.62 & 0.29 & 0.71 & 0.38 & 0 & 0 & 0.00 \\
\hline 152 & 303 & 1140 & 10 & 30 & 8 & 100 & 10.04 & 21.22 & 13.50 & 0.41 & 0.83 & 0.52 & 0 & 0 & 0.00 \\
\hline 154 & 403 & 1520 & 10 & 40 & 2 & 100 & 0.49 & 1.72 & 1.29 & 0.16 & 0.45 & 0.32 & 0 & 0 & 0.00 \\
\hline 156 & 403 & 1520 & 10 & 40 & 4 & 100 & 3.85 & 9.14 & 6.22 & 0.43 & 0.99 & 0.69 & 0 & 0 & 0.00 \\
\hline 158 & 403 & 1520 & 10 & 40 & 6 & 100 & 14.23 & 38.00 & 21.94 & 0.89 & 1.92 & 1.18 & 0 & 0 & 0.00 \\
\hline 160 & 403 & 1520 & 10 & 40 & 8 & 100 & 31.76 & 64.35 & 48.03 & 1.12 & 2.03 & 1.52 & 0 & 0 & 0.00 \\
\hline 162 & 203 & 780 & 20 & 10 & 2 & 100 & 0.01 & 0.03 & 0.02 & 0.00 & 0.01 & 0.01 & 0 & 1 & 0.05 \\
\hline 164 & 203 & 780 & 20 & 10 & 4 & 100 & 0.06 & 0.13 & 0.09 & 0.01 & 0.02 & 0.01 & 0 & 0 & 0.00 \\
\hline 166 & 203 & 780 & 20 & 10 & 6 & 100 & 0.16 & 0.23 & 0.20 & 0.02 & 0.03 & 0.02 & 0 & 0 & 0.00 \\
\hline 168 & 203 & 780 & 20 & 10 & 8 & 100 & 0.33 & 0.53 & 0.39 & 0.03 & 0.04 & 0.03 & 0 & 0 & 0.00 \\
\hline 170 & 403 & 1560 & 20 & 20 & 2 & 100 & 0.12 & 0.35 & 0.20 & 0.04 & 0.08 & 0.06 & 0 & 0 & 0.00 \\
\hline 172 & 403 & 1560 & 20 & 20 & 4 & 100 & 0.66 & 1.23 & 0.91 & 0.10 & 0.17 & 0.12 & 0 & 0 & 0.00 \\
\hline 174 & 403 & 1560 & 20 & 20 & 6 & 100 & 1.78 & 3.17 & 2.53 & 0.14 & 0.31 & 0.22 & 0 & 0 & 0.00 \\
\hline 176 & 403 & 1560 & 20 & 20 & 8 & 100 & 4.43 & 7.14 & 5.53 & 0.23 & 0.36 & 0.28 & 0 & 0 & 0.00 \\
\hline 178 & 603 & 2340 & 20 & 30 & 2 & 100 & 0.51 & 1.23 & 0.86 & 0.13 & 0.33 & 0.24 & 0 & 0 & 0.00 \\
\hline 180 & 603 & 2340 & 20 & 30 & 4 & 100 & 3.27 & 6.58 & 4.88 & 0.37 & 0.70 & 0.54 & 0 & 0 & 0.00 \\
\hline 182 & 603 & 2340 & 20 & 30 & 6 & 100 & 11.77 & 17.85 & 14.03 & 0.65 & 1.11 & 0.82 & 0 & 0 & 0.00 \\
\hline 184 & 603 & 2340 & 20 & 30 & 8 & 100 & 25.08 & 41.91 & 33.67 & 0.90 & 1.44 & 1.16 & 0 & 0 & 0.00 \\
\hline 186 & 803 & 3120 & 20 & 40 & 2 & 100 & 2.25 & 4.37 & 2.95 & 0.45 & 1.04 & 0.74 & 0 & 0 & 0.00 \\
\hline 188 & 803 & 3120 & 20 & 40 & 4 & 100 & 11.33 & 21.13 & 16.81 & 1.28 & 2.08 & 1.67 & 0 & 0 & 0.00 \\
\hline 190 & 803 & 3120 & 20 & 40 & 6 & 100 & 46.07 & 77.30 & 57.56 & 2.16 & 4.32 & 2.96 & 0 & 0 & 0.00 \\
\hline 192 & 803 & 3120 & 20 & 40 & 8 & 100 & 91.43 & 151.13 & 131.65 & 2.85 & 5.03 & 4.01 & 0 & 0 & 0.00 \\
\hline 194 & 303 & 1180 & 30 & 10 & 2 & 100 & 0.03 & 0.04 & 0.03 & 0.01 & 0.02 & 0.01 & 0 & 1 & 0.05 \\
\hline 196 & 303 & 1180 & 30 & 10 & 4 & 100 & 0.10 & 0.16 & 0.12 & 0.02 & 0.03 & 0.02 & 0 & 0 & 0.00 \\
\hline 198 & 303 & 1180 & 30 & 10 & 6 & 100 & 0.30 & 0.42 & 0.33 & 0.03 & 0.04 & 0.03 & 0 & 0 & 0.00 \\
\hline 200 & 303 & 1180 & 30 & 10 & 8 & 100 & 0.53 & 0.78 & 0.62 & 0.04 & 0.06 & 0.05 & 0 & 0 & 0.00 \\
\hline 202 & 603 & 2360 & 30 & 20 & 2 & 100 & 0.24 & 0.44 & 0.33 & 0.07 & 0.13 & 0.09 & 0 & 0 & 0.00 \\
\hline 204 & 603 & 2360 & 30 & 20 & 4 & 100 & 1.13 & 1.97 & 1.54 & 0.16 & 0.26 & 0.21 & 0 & 0 & 0.00 \\
\hline 206 & 603 & 2360 & 30 & 20 & 6 & 100 & 3.29 & 5.53 & 4.30 & 0.25 & 0.39 & 0.31 & 0 & 0 & 0.00 \\
\hline 208 & 603 & 2360 & 30 & 20 & 8 & 100 & 7.85 & 10.73 & 9.26 & 0.36 & 0.48 & 0.42 & 0 & 0 & 0.00 \\
\hline 210 & 903 & 3540 & 30 & 30 & 2 & 100 & 0.91 & 1.96 & 1.44 & 0.23 & 0.54 & 0.38 & 0 & 0 & 0.00 \\
\hline 212 & 903 & 3540 & 30 & 30 & 4 & 100 & 5.91 & 8.89 & 7.13 & 0.67 & 0.96 & 0.81 & 0 & 0 & 0.00 \\
\hline 214 & 903 & 3540 & 30 & 30 & 6 & 100 & 17.54 & 30.36 & 24.30 & 1.02 & 1.72 & 1.39 & 0 & 0 & 0.00 \\
\hline 216 & 903 & 3540 & 30 & 30 & 8 & 100 & 44.71 & 67.51 & 55.43 & 1.60 & 2.92 & 2.06 & 0 & 0 & 0.00 \\
\hline 218 & 1203 & 4720 & 30 & 40 & 2 & 100 & 3.71 & 6.72 & 4.93 & 0.79 & 1.92 & 1.29 & 0 & 0 & 0.00 \\
\hline 220 & 1203 & 4720 & 30 & 40 & 4 & 100 & 21.55 & 35.97 & 27.13 & 2.32 & 3.35 & 2.83 & 0 & 0 & 0.00 \\
\hline 222 & 1203 & 4720 & 30 & 40 & 6 & 100 & 75.70 & 115.70 & 89.83 & 3.93 & 7.95 & 5.38 & 0 & 0 & 0.00 \\
\hline 224 & 1203 & 4720 & 30 & 40 & 8 & 100 & 164.84 & 285.93 & 215.87 & 5.51 & 9.89 & 7.52 & 0 & 0 & 0.00 \\
\hline 226 & 403 & 1580 & 40 & 10 & 2 & 100 & 0.03 & 0.06 & 0.04 & 0.01 & 0.02 & 0.01 & 0 & 1 & 0.05 \\
\hline 228 & 403 & 1580 & 40 & 10 & 4 & 100 & 0.14 & 0.23 & 0.18 & 0.02 & 0.04 & 0.03 & 0 & 0 & 0.00 \\
\hline 230 & 403 & 1580 & 40 & 10 & 6 & 100 & 0.36 & 0.50 & 0.44 & 0.04 & 0.06 & 0.05 & 0 & 0 & 0.00 \\
\hline 232 & 403 & 1580 & 40 & 10 & 8 & 100 & 0.75 & 1.06 & 0.84 & 0.05 & 0.11 & 0.07 & 0 & 0 & 0.00 \\
\hline 234 & 803 & 3160 & 40 & 20 & 2 & 100 & 0.27 & 0.50 & 0.41 & 0.08 & 0.17 & 0.12 & 0 & 0 & 0.00 \\
\hline 236 & 803 & 3160 & 40 & 20 & 4 & 100 & 1.55 & 2.31 & 1.99 & 0.20 & 0.31 & 0.27 & 0 & 0 & 0.00 \\
\hline 238 & 803 & 3160 & 40 & 20 & 6 & 100 & 4.60 & 6.99 & 5.71 & 0.36 & 0.55 & 0.44 & 0 & 0 & 0.00 \\
\hline 240 & 803 & 3160 & 40 & 20 & 8 & 100 & 10.88 & 17.21 & 13.15 & 0.51 & 0.90 & 0.66 & 0 & 0 & 0.00 \\
\hline 242 & 1203 & 4740 & 40 & 30 & 2 & 100 & 1.47 & 2.43 & 1.96 & 0.38 & 0.72 & 0.50 & 0 & 0 & 0.00 \\
\hline 244 & 1203 & 4740 & 40 & 30 & 4 & 100 & 8.13 & 14.39 & 10.90 & 0.90 & 1.45 & 1.19 & 0 & 0 & 0.00 \\
\hline 246 & 1203 & 4740 & 40 & 30 & 6 & 100 & 23.58 & 39.68 & 31.96 & 1.47 & 2.22 & 1.84 & 0 & 0 & 0.00 \\
\hline 248 & 1203 & 4740 & 40 & 30 & 8 & 100 & 65.23 & 84.04 & 75.04 & 2.35 & 2.97 & 2.64 & 0 & 0 & 0.00 \\
\hline 250 & 1603 & 6320 & 40 & 40 & 2 & 100 & 4.45 & 8.06 & 6.20 & 1.08 & 2.13 & 1.51 & 0 & 0 & 0.00 \\
\hline 252 & 1603 & 6320 & 40 & 40 & 4 & 100 & 27.58 & 44.04 & 36.00 & 3.02 & 4.83 & 3.84 & 0 & 0 & 0.00 \\
\hline 254 & 1603 & 6320 & 40 & 40 & 6 & 100 & 98.10 & 147.79 & 122.18 & 5.42 & 7.37 & 6.26 & 0 & 0 & 0.00 \\
\hline 256 & 1603 & 6320 & 40 & 40 & 8 & 100 & 244.55 & 343.90 & 278.67 & 7.45 & 10.67 & 8.72 & 0 & 0 & 0.00 \\
\hline
\end{tabular}

https://doi.org/10.1002/net.21815 
A.2. Extensions of Labeling Algorithms

${ }^{38} \perp$ WILEY

RAITH ET AL

T A B LE A13 Grid instances with three objectives $(k=3)$, random scenarios and $c=10$

\begin{tabular}{|c|c|c|c|c|c|c|c|c|c|c|c|c|}
\hline & $|V|$ & $|E|$ & $h$ & $w$ & $r$ & $c$ & EL-Flimsily & RL-Flimsily & sols & EL-Highly & RL-Highly & sols \\
\hline 1026 & 103 & 380 & 10 & 10 & 2 & 10 & 0.02 & 0.01 & 45 & 0.02 & 0.01 & 0 \\
\hline 1028 & 103 & 380 & 10 & 10 & 4 & 10 & 0.08 & 0.02 & 93 & 0.08 & 0.01 & 0 \\
\hline 1030 & 103 & 380 & 10 & 10 & 6 & 10 & 0.25 & 0.03 & 146 & 0.24 & 0.04 & 0 \\
\hline 1032 & 103 & 380 & 10 & 10 & 8 & 10 & 0.44 & 0.04 & 186 & 0.43 & 0.04 & 0 \\
\hline 1034 & 203 & 760 & 10 & 20 & 2 & 10 & 0.60 & 0.17 & 228 & 0.61 & 0.17 & 1 \\
\hline 1036 & 203 & 760 & 10 & 20 & 4 & 10 & 2.29 & 0.25 & 401 & 2.25 & 0.26 & 0 \\
\hline 1038 & 203 & 760 & 10 & 20 & 6 & 10 & 8.81 & 0.57 & 496 & 8.43 & 0.51 & 0 \\
\hline 1040 & 203 & 760 & 10 & 20 & 8 & 10 & 17.28 & 0.64 & 781 & 17.37 & 0.63 & 0 \\
\hline 1042 & 303 & 1140 & 10 & 30 & 2 & 10 & 5.51 & 1.47 & 588 & 5.36 & 1.47 & 1 \\
\hline 1044 & 303 & 1140 & 10 & 30 & 4 & 10 & 33.68 & 2.70 & 932 & 27.06 & 2.68 & 0 \\
\hline 1046 & 303 & 1140 & 10 & 30 & 6 & 10 & 106.44 & 4.05 & 1220 & 104.26 & 4.05 & 0 \\
\hline 1048 & 303 & 1140 & 10 & 30 & 8 & 10 & 242.27 & 5.51 & 1642 & 237.83 & 5.48 & 0 \\
\hline 1050 & 403 & 1520 & 10 & 40 & 2 & 10 & 9.20 & 2.32 & 718 & 9.36 & 2.32 & 0 \\
\hline 1052 & 403 & 1520 & 10 & 40 & 4 & 10 & 148.36 & 10.65 & 1847 & 145.65 & 10.67 & 0 \\
\hline 1054 & 403 & 1520 & 10 & 40 & 6 & 10 & 491.12 & 16.51 & 2204 & 483.56 & 16.21 & 0 \\
\hline 1056 & 403 & 1520 & 10 & 40 & 8 & 10 & 1234.74 & 32.54 & 3295 & 1198.43 & 28.95 & 0 \\
\hline 1058 & 203 & 780 & 20 & 10 & 2 & 10 & 0.05 & 0.02 & 46 & 0.05 & 0.02 & 0 \\
\hline 1060 & 203 & 780 & 20 & 10 & 4 & 10 & 0.24 & 0.05 & 133 & 0.24 & 0.06 & 0 \\
\hline 1062 & 203 & 780 & 20 & 10 & 6 & 10 & 0.57 & 0.07 & 230 & 0.55 & 0.06 & 0 \\
\hline 1064 & 203 & 780 & 20 & 10 & 8 & 10 & 0.89 & 0.08 & 200 & 0.89 & 0.07 & 0 \\
\hline 1066 & 403 & 1560 & 20 & 20 & 2 & 10 & 1.31 & 0.40 & 205 & 1.28 & 0.32 & 0 \\
\hline 1068 & 403 & 1560 & 20 & 20 & 4 & 10 & 6.64 & 0.90 & 434 & 6.76 & 0.73 & 0 \\
\hline 1070 & 403 & 1560 & 20 & 20 & 6 & 10 & 20.80 & 1.26 & 679 & 21.07 & 1.13 & 0 \\
\hline 1072 & 403 & 1560 & 20 & 20 & 8 & 10 & 44.36 & 1.60 & 818 & 43.00 & 1.42 & 0 \\
\hline 1074 & 603 & 2340 & 20 & 30 & 2 & 10 & 14.66 & 3.71 & 610 & 13.91 & 3.74 & 0 \\
\hline 1076 & 603 & 2340 & 20 & 30 & 4 & 10 & 74.66 & 5.88 & 928 & 66.94 & 5.96 & 0 \\
\hline 1078 & 603 & 2340 & 20 & 30 & 6 & 10 & 352.76 & 16.43 & 1415 & 315.07 & 12.62 & 0 \\
\hline 1080 & 603 & 2340 & 20 & 30 & 8 & 10 & 568.69 & 13.23 & 1798 & 527.84 & 12.80 & 0 \\
\hline 1082 & 803 & 3120 & 20 & 40 & 2 & 10 & 111.45 & 30.58 & 1150 & 91.68 & 25.05 & 0 \\
\hline 1084 & 803 & 3120 & 20 & 40 & 4 & 10 & 418.06 & 31.76 & 1839 & 357.96 & 28.81 & 0 \\
\hline 1086 & 803 & 3120 & 20 & 40 & 6 & 10 & 1952.35 & 79.62 & 2729 & 1636.52 & 68.01 & 0 \\
\hline 1088 & 803 & 3120 & 20 & 40 & 8 & 10 & $>3600.00$ & 97.92 & 4023 & 3512.34 & 85.67 & 0 \\
\hline 1090 & 303 & 1180 & 30 & 10 & 2 & 10 & 0.11 & 0.03 & 59 & 0.09 & 0.03 & 0 \\
\hline 1092 & 303 & 1180 & 30 & 10 & 4 & 10 & 0.25 & 0.04 & 92 & 0.25 & 0.04 & 0 \\
\hline 1094 & 303 & 1180 & 30 & 10 & 6 & 10 & 0.80 & 0.09 & 165 & 0.80 & 0.08 & 0 \\
\hline 1096 & 303 & 1180 & 30 & 10 & 8 & 10 & 1.94 & 0.14 & 304 & 1.89 & 0.13 & 0 \\
\hline 1098 & 603 & 2360 & 30 & 20 & 2 & 10 & 3.25 & 0.68 & 311 & 2.77 & 0.69 & 0 \\
\hline 1100 & 603 & 2360 & 30 & 20 & 4 & 10 & 12.44 & 1.20 & 395 & 10.93 & 1.19 & 0 \\
\hline 1102 & 603 & 2360 & 30 & 20 & 6 & 10 & 38.77 & 1.76 & 644 & 33.41 & 1.83 & 0 \\
\hline 1104 & 603 & 2360 & 30 & 20 & 8 & 10 & 83.17 & 2.54 & 760 & 77.84 & 2.47 & 0 \\
\hline 1106 & 903 & 3540 & 30 & 30 & 2 & 10 & 16.44 & 4.97 & 530 & 16.46 & 4.30 & 0 \\
\hline 1108 & 903 & 3540 & 30 & 30 & 4 & 10 & 132.24 & 15.91 & 1033 & 129.25 & 13.25 & 0 \\
\hline 1110 & 903 & 3540 & 30 & 30 & 6 & 10 & 488.53 & 25.34 & 1733 & 504.64 & 21.43 & 0 \\
\hline 1112 & 903 & 3540 & 30 & 30 & 8 & 10 & 813.58 & 28.53 & 2040 & 868.63 & 23.06 & 0 \\
\hline 1114 & 1203 & 4720 & 30 & 40 & 2 & 10 & 100.13 & 39.45 & 959 & 105.10 & 33.00 & 0 \\
\hline 1116 & 1203 & 4720 & 30 & 40 & 4 & 10 & 665.71 & 82.27 & 2203 & 765.86 & 73.64 & 0 \\
\hline 1118 & 1203 & 4720 & 30 & 40 & 6 & 10 & $>3600.00$ & 230.81 & 3432 & $>3600.00$ & 202.97 & 0 \\
\hline 1120 & 1203 & 4720 & 30 & 40 & 8 & 10 & $>3600.00$ & 219.66 & 4489 & $>3600.00$ & 195.73 & 0 \\
\hline 1122 & 403 & 1580 & 40 & 10 & 2 & 10 & 0.13 & 0.04 & 75 & 0.12 & 0.05 & 0 \\
\hline 1124 & 403 & 1580 & 40 & 10 & 4 & 10 & 0.47 & 0.07 & 158 & 0.48 & 0.07 & 0 \\
\hline 1126 & 403 & 1580 & 40 & 10 & 6 & 10 & 1.23 & 0.12 & 181 & 1.26 & 0.12 & 0 \\
\hline 1128 & 403 & 1580 & 40 & 10 & 8 & 10 & 2.30 & 0.16 & 295 & 2.39 & 0.16 & 0 \\
\hline 1130 & 803 & 3160 & 40 & 20 & 2 & 10 & 2.61 & 0.88 & 219 & 2.67 & 0.73 & 2 \\
\hline 1132 & 803 & 3160 & 40 & 20 & 4 & 10 & 14.67 & 2.08 & 540 & 15.43 & 1.70 & 0 \\
\hline 1134 & 803 & 3160 & 40 & 20 & 6 & 10 & 56.70 & 3.25 & 951 & 60.93 & 3.14 & 0 \\
\hline 1136 & 803 & 3160 & 40 & 20 & 8 & 10 & 92.92 & 3.49 & 1014 & 100.45 & 3.15 & 0 \\
\hline 1138 & 1203 & 4740 & 40 & 30 & 2 & 10 & 18.61 & 5.29 & 495 & 19.57 & 5.31 & 0 \\
\hline 1140 & 1203 & 4740 & 40 & 30 & 4 & 10 & 188.02 & 23.59 & 1347 & 206.80 & 20.02 & 0 \\
\hline 1142 & 1203 & 4740 & 40 & 30 & 6 & 10 & 489.84 & 29.04 & 1547 & 518.47 & 24.07 & 0 \\
\hline 1144 & 1203 & 4740 & 40 & 30 & 8 & 10 & 1165.91 & 37.14 & 2020 & 1256.95 & 35.74 & 0 \\
\hline 1146 & 1603 & 6320 & 40 & 40 & 2 & 10 & 99.04 & 40.55 & 943 & 102.94 & 32.93 & 0 \\
\hline 1148 & 1603 & 6320 & 40 & 40 & 4 & 10 & 1093.01 & 141.50 & 2035 & 1185.97 & 120.75 & 0 \\
\hline 1150 & 1603 & 6320 & 40 & 40 & 6 & 10 & $>3600.00$ & 256.02 & 3829 & $>3600.00$ & 238.18 & 0 \\
\hline 1152 & 1603 & 6320 & 40 & 40 & 8 & 10 & $>3600.00$ & 264.21 & 3624 & $>3600.00$ & 246.88 & 0 \\
\hline
\end{tabular}

https://doi.org/10.1002/net.21815 
A. Publications

RAITH ET AL.

WILEY

T A B LE A14 Grid instances with three objectives $(k=3)$, random scenarios and $c=100$

\begin{tabular}{|c|c|c|c|c|c|c|c|c|c|c|c|c|}
\hline & $|V|$ & $|E|$ & $\boldsymbol{h}$ & $w$ & $r$ & $c$ & EL-Flimsily & RL-Flimsily & sols & EL-Highly & RL-Highly & sols \\
\hline 1154 & 103 & 380 & 10 & 10 & 2 & 100 & 0.04 & 0.01 & 72 & 0.04 & 0.01 & 3 \\
\hline 1156 & 103 & 380 & 10 & 10 & 4 & 100 & 0.23 & 0.04 & 177 & 0.24 & 0.04 & 0 \\
\hline 1158 & 103 & 380 & 10 & 10 & 6 & 100 & 0.56 & 0.05 & 283 & 0.56 & 0.05 & 0 \\
\hline 1160 & 103 & 380 & 10 & 10 & 8 & 100 & 0.81 & 0.06 & 309 & 0.86 & 0.07 & 0 \\
\hline 1162 & 203 & 760 & 10 & 20 & 2 & 100 & 1.35 & 0.39 & 337 & 1.47 & 0.39 & 6 \\
\hline 1164 & 203 & 760 & 10 & 20 & 4 & 100 & 7.87 & 0.81 & 640 & 8.95 & 0.81 & 0 \\
\hline 1166 & 203 & 760 & 10 & 20 & 6 & 100 & 26.56 & 1.39 & 952 & 34.07 & 1.38 & 0 \\
\hline 1168 & 203 & 760 & 10 & 20 & 8 & 100 & 63.08 & 1.98 & 1272 & 74.09 & 1.95 & 0 \\
\hline 1170 & 303 & 1140 & 10 & 30 & 2 & 100 & 20.27 & 3.87 & 716 & 22.95 & 3.82 & 0 \\
\hline 1172 & 303 & 1140 & 10 & 30 & 4 & 100 & 66.37 & 5.58 & 1388 & 77.13 & 5.55 & 0 \\
\hline 1174 & 303 & 1140 & 10 & 30 & 6 & 100 & 684.83 & 22.33 & 2702 & 765.73 & 22.19 & 0 \\
\hline 1176 & 303 & 1140 & 10 & 30 & 8 & 100 & 873.13 & 21.27 & 4133 & 1012.57 & 20.86 & 0 \\
\hline 1178 & 403 & 1520 & 10 & 40 & 2 & 100 & 146.11 & 38.21 & 2300 & 165.53 & 37.03 & 0 \\
\hline 1180 & 403 & 1520 & 10 & 40 & 4 & 100 & 775.24 & 68.38 & 3471 & 901.80 & 68.28 & 0 \\
\hline 1182 & 403 & 1520 & 10 & 40 & 6 & 100 & 2506.19 & 90.50 & 4589 & 2823.64 & 112.26 & 0 \\
\hline 1184 & 403 & 1520 & 10 & 40 & 8 & 100 & $>3600.00$ & 142.47 & 6759 & $>3600.00$ & 135.13 & 0 \\
\hline 1186 & 203 & 780 & 20 & 10 & 2 & 100 & 0.14 & 0.04 & 109 & 0.13 & 0.05 & 0 \\
\hline 1188 & 203 & 780 & 20 & 10 & 4 & 100 & 0.57 & 0.08 & 205 & 0.56 & 0.08 & 0 \\
\hline 1190 & 203 & 780 & 20 & 10 & 6 & 100 & 1.30 & 0.14 & 303 & 1.29 & 0.13 & 0 \\
\hline 1192 & 203 & 780 & 20 & 10 & 8 & 100 & 2.91 & 0.21 & 394 & 2.91 & 0.18 & 0 \\
\hline 1194 & 403 & 1560 & 20 & 20 & 2 & 100 & 2.07 & 0.62 & 273 & 2.07 & 0.56 & 0 \\
\hline 1196 & 403 & 1560 & 20 & 20 & 4 & 100 & 16.12 & 2.21 & 648 & 16.34 & 1.68 & 0 \\
\hline 1198 & 403 & 1560 & 20 & 20 & 6 & 100 & 73.50 & 5.42 & 1266 & 73.70 & 3.17 & 0 \\
\hline 1200 & 403 & 1560 & 20 & 20 & 8 & 100 & 244.92 & 6.70 & 1735 & 222.19 & 5.99 & 0 \\
\hline 1202 & 603 & 2340 & 20 & 30 & 2 & 100 & 55.67 & 16.18 & 1163 & 46.58 & 13.73 & 0 \\
\hline 1204 & 603 & 2340 & 20 & 30 & 4 & 100 & 333.06 & 30.67 & 1882 & 282.90 & 26.70 & 0 \\
\hline 1206 & 603 & 2340 & 20 & 30 & 6 & 100 & 1620.06 & 63.09 & 3099 & 1436.10 & 58.27 & 0 \\
\hline 1208 & 603 & 2340 & 20 & 30 & 8 & 100 & 2055.06 & 53.99 & 3228 & 1843.03 & 49.93 & 0 \\
\hline 1210 & 803 & 3120 & 20 & 40 & 2 & 100 & 550.67 & 143.66 & 2839 & 521.30 & 138.06 & 0 \\
\hline 1212 & 803 & 3120 & 20 & 40 & 4 & 100 & 2426.44 & 262.89 & 4411 & 2358.97 & 256.39 & 0 \\
\hline 1214 & 803 & 3120 & 20 & 40 & 6 & 100 & $>3600.00$ & 360.39 & 6602 & $>3600.00$ & 340.70 & 0 \\
\hline 1216 & 803 & 3120 & 20 & 40 & 8 & 100 & $>3600.00$ & 622.66 & 7463 & $>3600.00$ & 585.15 & 0 \\
\hline 1218 & 303 & 1180 & 30 & 10 & 2 & 100 & 0.19 & 0.05 & 115 & 0.17 & 0.05 & 3 \\
\hline 1220 & 303 & 1180 & 30 & 10 & 4 & 100 & 0.67 & 0.10 & 179 & 0.66 & 0.10 & 0 \\
\hline 1222 & 303 & 1180 & 30 & 10 & 6 & 100 & 2.23 & 0.19 & 282 & 2.14 & 0.18 & 0 \\
\hline 1224 & 303 & 1180 & 30 & 10 & 8 & 100 & 4.05 & 0.25 & 418 & 4.00 & 0.25 & 0 \\
\hline 1226 & 603 & 2360 & 30 & 20 & 2 & 100 & 5.04 & 1.33 & 421 & 4.96 & 1.29 & 1 \\
\hline 1228 & 603 & 2360 & 30 & 20 & 4 & 100 & 40.44 & 3.81 & 1008 & 39.30 & 3.70 & 0 \\
\hline 1230 & 603 & 2360 & 30 & 20 & 6 & 100 & 122.85 & 5.67 & 1311 & 118.17 & 5.60 & 0 \\
\hline 1232 & 603 & 2360 & 30 & 20 & 8 & 100 & 328.83 & 8.38 & 1544 & 308.09 & 8.15 & 0 \\
\hline 1234 & 903 & 3540 & 30 & 30 & 2 & 100 & 77.84 & 24.07 & 901 & 76.46 & 23.83 & 0 \\
\hline 1236 & 903 & 3540 & 30 & 30 & 4 & 100 & 649.61 & 63.32 & 2138 & 615.86 & 62.24 & 0 \\
\hline 1238 & 903 & 3540 & 30 & 30 & 6 & 100 & 2100.36 & 91.28 & 3188 & 1992.99 & 89.51 & 0 \\
\hline 1240 & 903 & 3540 & 30 & 30 & 8 & 100 & $>3600.00$ & 147.84 & 4534 & $>3600.00$ & 144.45 & 0 \\
\hline 1242 & 1203 & 4720 & 30 & 40 & 2 & 100 & 915.71 & 246.27 & 2450 & 870.75 & 239.12 & 0 \\
\hline 1244 & 1203 & 4720 & 30 & 40 & 4 & 100 & $>3600.00$ & 438.21 & 4175 & $>3600.00$ & 427.43 & 0 \\
\hline 1246 & 1203 & 4720 & 30 & 40 & 6 & 100 & $>3600.00$ & 788.21 & 7070 & $>3600.00$ & 764.83 & 0 \\
\hline 1248 & 1203 & 4720 & 30 & 40 & 8 & 100 & $>3600.00$ & 818.04 & 7736 & $>3600.00$ & 816.31 & 0 \\
\hline 1250 & 403 & 1580 & 40 & 10 & 2 & 100 & 0.24 & 0.07 & 102 & 0.24 & 0.07 & 2 \\
\hline 1252 & 403 & 1580 & 40 & 10 & 4 & 100 & 1.22 & 0.17 & 221 & 1.14 & 0.17 & 0 \\
\hline 1254 & 403 & 1580 & 40 & 10 & 6 & 100 & 3.92 & 0.25 & 342 & 3.37 & 0.25 & 0 \\
\hline 1256 & 403 & 1580 & 40 & 10 & 8 & 100 & 7.59 & 0.40 & 527 & 7.10 & 0.38 & 0 \\
\hline 1258 & 803 & 3160 & 40 & 20 & 2 & 100 & 12.08 & 2.42 & 480 & 9.08 & 2.42 & 1 \\
\hline 1260 & 803 & 3160 & 40 & 20 & 4 & 100 & 57.09 & 5.22 & 1191 & 52.62 & 5.10 & 0 \\
\hline 1262 & 803 & 3160 & 40 & 20 & 6 & 100 & 195.43 & 9.23 & 1377 & 194.14 & 9.05 & 0 \\
\hline 1264 & 803 & 3160 & 40 & 20 & 8 & 100 & 424.89 & 11.31 & 2014 & 405.64 & 11.84 & 0 \\
\hline 1266 & 1203 & 4740 & 40 & 30 & 2 & 100 & 98.15 & 31.98 & 842 & 94.77 & 31.18 & 0 \\
\hline 1268 & 1203 & 4740 & 40 & 30 & 4 & 100 & 949.93 & 95.71 & 2942 & 895.28 & 96.66 & 0 \\
\hline 1270 & 1203 & 4740 & 40 & 30 & 6 & 100 & 3143.94 & 145.54 & 3410 & 2893.93 & 145.00 & 0 \\
\hline 1272 & 1203 & 4740 & 40 & 30 & 8 & 100 & $>3600.00$ & 177.09 & 4526 & $>3600.00$ & 175.63 & 0 \\
\hline 1274 & 1603 & 6320 & 40 & 40 & 2 & 100 & 713.25 & 217.56 & 1957 & 771.89 & 215.14 & 0 \\
\hline 1276 & 1603 & 6320 & 40 & 40 & 4 & 100 & $>3600.00$ & 689.56 & 4485 & $>3600.00$ & 693.92 & 0 \\
\hline 1278 & 1603 & 6320 & 40 & 40 & 6 & 100 & $>3600.00$ & 858.13 & 6418 & $>3600.00$ & 852.94 & 0 \\
\hline 1280 & 1603 & 6320 & 40 & 40 & 8 & 100 & $>3600.00$ & 1369.15 & 8956 & $>3600.00$ & 1251.78 & 0 \\
\hline
\end{tabular}

https://doi.org/10.1002/net.21815 
A.2. Extensions of Labeling Algorithms

$\stackrel{40}{ }{ }^{\text {WI LEY }}$

RAITH ET AL.

T A B L E A15 Grid instances with four objectives $(k=4)$, random scenarios and $c=10$

\begin{tabular}{|c|c|c|c|c|c|c|c|c|c|c|c|c|}
\hline & $|V|$ & $|E|$ & $h$ & $w$ & $r$ & $c$ & EL-Flimsily & RL-Flimsily & sols & EL-Highly & RL-Highly & sols \\
\hline 2051 & 103 & 380 & 10 & 10 & 2 & 10 & 0.06 & 0.02 & 85 & 0.06 & 0.02 & 4 \\
\hline 2053 & 103 & 380 & 10 & 10 & 4 & 10 & 0.32 & 0.05 & 261 & 0.30 & 0.04 & 0 \\
\hline 2055 & 203 & 760 & 10 & 20 & 2 & 10 & 4.57 & 1.26 & 702 & 4.60 & 1.18 & 13 \\
\hline 2057 & 203 & 760 & 10 & 20 & 4 & 10 & 18.24 & 1.69 & 1200 & 17.84 & 1.62 & 0 \\
\hline 2059 & 303 & 1140 & 10 & 30 & 2 & 10 & 63.72 & 13.95 & 2250 & 64.74 & 13.87 & 3 \\
\hline 2061 & 303 & 1140 & 10 & 30 & 4 & 10 & 704.48 & 47.53 & 4365 & 713.43 & 47.02 & 0 \\
\hline 2063 & 203 & 780 & 20 & 10 & 2 & 10 & 0.19 & 0.05 & 182 & 0.19 & 0.06 & 12 \\
\hline 2065 & 203 & 780 & 20 & 10 & 4 & 10 & 0.83 & 0.11 & 274 & 0.79 & 0.11 & 0 \\
\hline 2067 & 403 & 1560 & 20 & 20 & 2 & 10 & 9.31 & 2.26 & 826 & 9.42 & 2.21 & 4 \\
\hline 2069 & 403 & 1560 & 20 & 20 & 4 & 10 & 71.01 & 5.85 & 1318 & 72.11 & 5.83 & 0 \\
\hline 2071 & 603 & 2340 & 20 & 30 & 2 & 10 & 242.44 & 50.55 & 2902 & 239.59 & 50.36 & 0 \\
\hline 2073 & 603 & 2340 & 20 & 30 & 4 & 10 & 2188.69 & 179.08 & 6114 & 2108.02 & 175.02 & 0 \\
\hline 2075 & 303 & 1180 & 30 & 10 & 2 & 10 & 0.35 & 0.09 & 207 & 0.34 & 0.09 & 10 \\
\hline 2077 & 303 & 1180 & 30 & 10 & 4 & 10 & 1.29 & 0.18 & 356 & 1.28 & 0.18 & 1 \\
\hline 2079 & 603 & 2360 & 30 & 20 & 2 & 10 & 10.27 & 2.61 & 559 & 10.33 & 2.56 & 0 \\
\hline 2081 & 603 & 2360 & 30 & 20 & 4 & 10 & 118.85 & 9.88 & 1851 & 117.38 & 10.14 & 0 \\
\hline 2083 & 903 & 3540 & 30 & 30 & 2 & 10 & 679.99 & 164.50 & 3121 & 657.14 & 164.40 & 2 \\
\hline 2085 & 903 & 3540 & 30 & 30 & 4 & 10 & 2468.86 & 205.38 & 3757 & 2392.72 & 207.28 & 0 \\
\hline
\end{tabular}

T A B LE A16 Grid instances with four objectives $(k=4)$, random scenarios and $c=100$

\begin{tabular}{|c|c|c|c|c|c|c|c|c|c|c|c|c|}
\hline & $|V|$ & $|E|$ & $\boldsymbol{h}$ & $w$ & $r$ & $c$ & EL-Flimsily & RL-Flimsily & sols & EL-Highly & RL-Highly & sols \\
\hline 2087 & 103 & 380 & 10 & 10 & 2 & 100 & 0.23 & 0.06 & 211 & 0.22 & 0.06 & 15 \\
\hline 2089 & 103 & 380 & 10 & 10 & 4 & 100 & 0.98 & 0.14 & 482 & 0.97 & 0.13 & 2 \\
\hline 2091 & 203 & 760 & 10 & 20 & 2 & 100 & 20.17 & 4.54 & 1143 & 19.46 & 4.45 & 15 \\
\hline 2093 & 203 & 760 & 10 & 20 & 4 & 100 & 138.79 & 10.59 & 2423 & 141.79 & 10.37 & 1 \\
\hline 2095 & 303 & 1140 & 10 & 30 & 2 & 100 & 339.03 & 68.72 & 4259 & 339.92 & 68.50 & 0 \\
\hline 2097 & 303 & 1140 & 10 & 30 & 4 & 100 & 3285.91 & 264.25 & 9803 & 3225.54 & 268.28 & 0 \\
\hline 2099 & 203 & 780 & 20 & 10 & 2 & 100 & 0.48 & 0.12 & 284 & 0.45 & 0.12 & 7 \\
\hline 2101 & 203 & 780 & 20 & 10 & 4 & 100 & 2.56 & 0.30 & 528 & 2.51 & 0.29 & 0 \\
\hline 2103 & 403 & 1560 & 20 & 20 & 2 & 100 & 59.49 & 13.63 & 1585 & 57.25 & 13.60 & 6 \\
\hline 2105 & 403 & 1560 & 20 & 20 & 4 & 100 & 407.55 & 29.63 & 2987 & 391.00 & 29.57 & 0 \\
\hline 2107 & 603 & 2340 & 20 & 30 & 2 & 100 & 1292.27 & 307.90 & 4996 & 1323.37 & 294.51 & 1 \\
\hline 2109 & 603 & 2340 & 20 & 30 & 4 & 100 & $>3600.00$ & 723.44 & 9834 & $>3600.00$ & 724.34 & 0 \\
\hline 2111 & 303 & 1180 & 30 & 10 & 2 & 100 & 0.93 & 0.21 & 347 & 0.81 & 0.21 & 14 \\
\hline 2113 & 303 & 1180 & 30 & 10 & 4 & 100 & 4.17 & 0.50 & 763 & 4.22 & 0.47 & 0 \\
\hline 2115 & 603 & 2360 & 30 & 20 & 2 & 100 & 90.71 & 24.55 & 1861 & 96.69 & 23.35 & 0 \\
\hline 2117 & 603 & 2360 & 30 & 20 & 4 & 100 & 361.71 & 31.64 & 3139 & 397.64 & 31.66 & 0 \\
\hline 2119 & 903 & 3540 & 30 & 30 & 2 & 100 & 1843.49 & 500.46 & 2588 & 1911.63 & 480.27 & 0 \\
\hline 2121 & 903 & 3540 & 30 & 30 & 4 & 100 & $>3600.00$ & 1593.80 & 12188 & $>3600.00$ & 1585.40 & 0 \\
\hline
\end{tabular}

https://doi.org/10.1002/net.21815 
RAITH ET AL.

T A B L E A17 NetMaker instances, flimsily robust efficiency, $k=2$, correlated scenarios

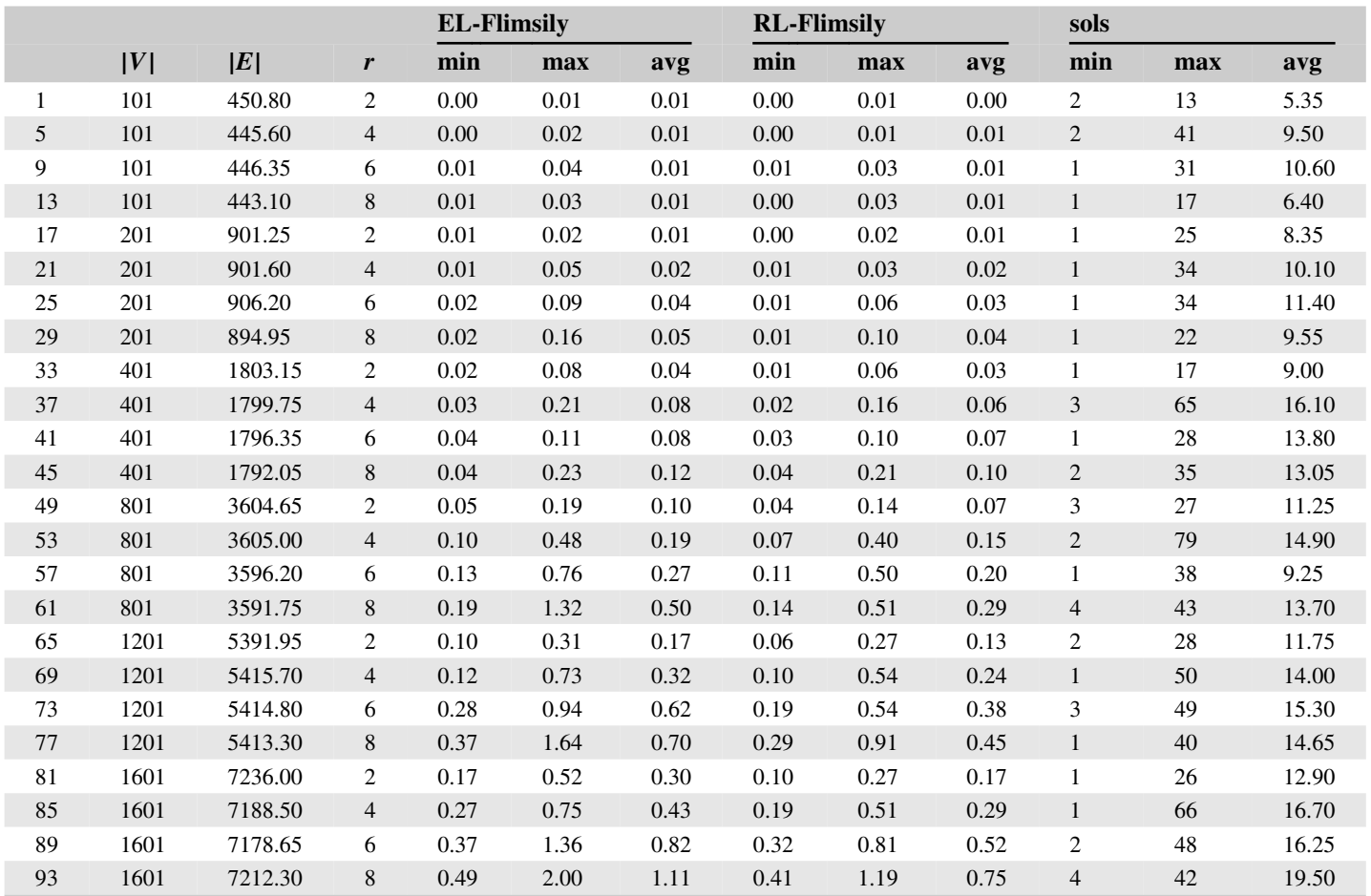

T A B L E $A 18$ NetMaker instances, flimsily robust efficiency, $k=3$, correlated scenarios

\begin{tabular}{|c|c|c|c|c|c|c|c|c|c|c|c|c|}
\hline & \multirow[b]{2}{*}{$|V|$} & \multirow[b]{2}{*}{$|E|$} & \multirow[b]{2}{*}{$r$} & \multicolumn{3}{|c|}{ EL-Flimsily } & \multicolumn{3}{|c|}{ RL-Flimsily } & \multicolumn{3}{|l|}{ sols } \\
\hline & & & & $\min$ & $\max$ & avg & $\min$ & $\max$ & avg & $\min$ & $\max$ & avg \\
\hline 2 & 101 & 446.90 & 2 & 0.04 & 0.62 & 0.30 & 0.03 & 0.53 & 0.26 & 22 & 134 & 65.50 \\
\hline 6 & 101 & 450.50 & 4 & 0.24 & 1.92 & 0.65 & 0.26 & 1.12 & 0.57 & 27 & 276 & 92.80 \\
\hline 10 & 101 & 445.50 & 6 & 0.15 & 2.67 & 1.08 & 0.23 & 2.95 & 1.19 & 19 & 422 & 169.60 \\
\hline 14 & 101 & 447.00 & 8 & 0.27 & 2.12 & 0.85 & 0.34 & 2.29 & 1.00 & 33 & 213 & 95.50 \\
\hline 18 & 201 & 913.20 & 2 & 0.36 & 2.90 & 1.41 & 0.33 & 2.13 & 1.09 & 45 & 266 & 140.40 \\
\hline 22 & 201 & 892.60 & 4 & 0.31 & 4.65 & 1.93 & 0.43 & 4.11 & 1.93 & 81 & 322 & 146.50 \\
\hline 26 & 201 & 906.70 & 6 & 0.68 & 6.62 & 2.59 & 0.90 & 5.76 & 2.72 & 10 & 239 & 99.90 \\
\hline 30 & 201 & 908.70 & 8 & 0.54 & 10.87 & 3.96 & 0.63 & 9.05 & 3.88 & 49 & 415 & 144.50 \\
\hline 34 & 401 & 1818.60 & 2 & 2.01 & 8.78 & 4.44 & 1.29 & 7.30 & 3.30 & 22 & 273 & 136.40 \\
\hline 38 & 401 & 1781.60 & 4 & 1.95 & 18.87 & 8.83 & 1.91 & 16.93 & 8.36 & 14 & 354 & 153.80 \\
\hline 42 & 401 & 1784.90 & 6 & 4.22 & 16.29 & 8.80 & 4.04 & 15.25 & 8.63 & 28 & 412 & 171.50 \\
\hline 46 & 401 & 1813.50 & 8 & 2.94 & 45.83 & 17.45 & 3.69 & 38.52 & 17.21 & 32 & 182 & 110.20 \\
\hline 50 & 801 & 3615.00 & 2 & 5.58 & 64.93 & 20.88 & 5.00 & 53.73 & 17.87 & 5 & 455 & 112.70 \\
\hline 54 & 801 & 3590.20 & 4 & 11.10 & 52.23 & 29.29 & 13.65 & 58.51 & 33.49 & 39 & 385 & 219.60 \\
\hline 58 & 801 & 3611.40 & 6 & 24.79 & 84.01 & 44.97 & 25.10 & 86.58 & 49.04 & 81 & 554 & 243.40 \\
\hline 62 & 801 & 3604.00 & 8 & 23.66 & 122.16 & 71.22 & 28.79 & 156.65 & 89.46 & 14 & 426 & 235.00 \\
\hline 66 & 1201 & 5399.90 & 2 & 14.66 & 68.65 & 34.96 & 14.53 & 61.97 & 33.86 & 33 & 322 & 196.60 \\
\hline 70 & 1201 & 5390.20 & 4 & 25.41 & 294.71 & 98.39 & 34.90 & 373.71 & 129.72 & 62 & 675 & 337.40 \\
\hline 74 & 1201 & 5422.00 & 6 & 43.57 & 240.33 & 107.85 & 61.74 & 288.51 & 144.40 & 68 & 1207 & 335.00 \\
\hline 78 & 1201 & 5406.00 & 8 & 33.10 & 262.59 & 134.06 & 50.84 & 397.31 & 196.54 & 60 & 1229 & 479.40 \\
\hline
\end{tabular}

https://doi.org/10.1002/net. 21815 
A.2. Extensions of Labeling Algorithms

$\stackrel{42}{\perp}$ WI LEY

RAITH ET AL.

T A B L E A19 NetMaker instances, highly robust efficiency, $k=2$, correlated scenarios

\begin{tabular}{|c|c|c|c|c|c|c|c|c|c|c|c|c|}
\hline & \multirow[b]{2}{*}{$|V|$} & \multirow[b]{2}{*}{$|E|$} & \multirow[b]{2}{*}{$r$} & \multicolumn{3}{|c|}{$\underline{\text { EL-Highly }}$} & \multicolumn{3}{|c|}{ RL-Highly } & \multicolumn{3}{|l|}{ sols } \\
\hline & & & & $\overline{\min }$ & $\max$ & $\overline{\text { avg }}$ & $\overline{\min }$ & $\max$ & $\overline{\text { avg }}$ & $\overline{\min }$ & $\max$ & $\overline{\text { avg }}$ \\
\hline 1 & 101 & 450.80 & 2 & 0.00 & 0.01 & 0.01 & 0.00 & 0.01 & 0.00 & 2 & 10 & 4.40 \\
\hline 5 & 101 & 445.60 & 4 & 0.00 & 0.02 & 0.01 & 0.00 & 0.01 & 0.01 & 2 & 10 & 4.75 \\
\hline 9 & 101 & 446.35 & 6 & 0.01 & 0.04 & 0.01 & 0.01 & 0.03 & 0.01 & 1 & 12 & 4.85 \\
\hline 13 & 101 & 443.10 & 8 & 0.01 & 0.03 & 0.01 & 0.00 & 0.02 & 0.01 & 1 & 9 & 3.75 \\
\hline 17 & 201 & 901.25 & 2 & 0.01 & 0.02 & 0.01 & 0.00 & 0.02 & 0.01 & 1 & 18 & 6.25 \\
\hline 21 & 201 & 901.60 & 4 & 0.01 & 0.05 & 0.02 & 0.01 & 0.04 & 0.02 & 1 & 13 & 6.15 \\
\hline 25 & 201 & 906.20 & 6 & 0.02 & 0.10 & 0.04 & 0.01 & 0.06 & 0.03 & 1 & 13 & 5.00 \\
\hline 29 & 201 & 894.95 & 8 & 0.02 & 0.16 & 0.05 & 0.01 & 0.11 & 0.04 & 1 & 8 & 3.95 \\
\hline 33 & 401 & 1803.15 & 2 & 0.02 & 0.08 & 0.04 & 0.01 & 0.06 & 0.03 & 1 & 14 & 7.30 \\
\hline 37 & 401 & 1799.75 & 4 & 0.03 & 0.21 & 0.08 & 0.02 & 0.17 & 0.06 & 2 & 14 & 6.45 \\
\hline 41 & 401 & 1796.35 & 6 & 0.04 & 0.11 & 0.08 & 0.03 & 0.11 & 0.06 & 1 & 12 & 5.80 \\
\hline 45 & 401 & 1792.05 & 8 & 0.04 & 0.24 & 0.11 & 0.04 & 0.17 & 0.09 & 1 & 13 & 4.80 \\
\hline 49 & 801 & 3604.65 & 2 & 0.05 & 0.19 & 0.10 & 0.04 & 0.12 & 0.07 & 2 & 22 & 8.30 \\
\hline 53 & 801 & 3605.00 & 4 & 0.10 & 0.46 & 0.19 & 0.08 & 0.36 & 0.15 & 1 & 22 & 6.10 \\
\hline 57 & 801 & 3596.20 & 6 & 0.14 & 0.75 & 0.27 & 0.10 & 0.46 & 0.20 & 1 & 8 & 3.60 \\
\hline 61 & 801 & 3591.75 & 8 & 0.19 & 1.31 & 0.49 & 0.15 & 0.52 & 0.30 & 0 & 11 & 5.15 \\
\hline 65 & 1201 & 5391.95 & 2 & 0.10 & 0.31 & 0.17 & 0.06 & 0.22 & 0.11 & 2 & 19 & 7.90 \\
\hline 69 & 1201 & 5415.70 & 4 & 0.13 & 0.73 & 0.32 & 0.10 & 0.42 & 0.21 & 1 & 15 & 6.50 \\
\hline 73 & 1201 & 5414.80 & 6 & 0.30 & 0.99 & 0.66 & 0.19 & 0.55 & 0.38 & 2 & 18 & 6.05 \\
\hline 77 & 1201 & 5413.30 & 8 & 0.37 & 1.60 & 0.72 & 0.30 & 0.93 & 0.48 & 1 & 9 & 5.15 \\
\hline 81 & 1601 & 7236.00 & 2 & 0.15 & 0.50 & 0.28 & 0.10 & 0.26 & 0.17 & 1 & 18 & 9.20 \\
\hline 85 & 1601 & 7188.50 & 4 & 0.27 & 0.69 & 0.42 & 0.20 & 0.53 & 0.34 & 1 & 15 & 6.90 \\
\hline 89 & 1601 & 7178.65 & 6 & 0.37 & 1.35 & 0.81 & 0.32 & 0.94 & 0.57 & 2 & 16 & 5.70 \\
\hline 93 & 1601 & 7212.30 & 8 & 0.52 & 2.07 & 1.13 & 0.41 & 1.20 & 0.77 & 3 & 16 & 7.00 \\
\hline
\end{tabular}

T A B L E A20 NetMaker instances, highly robust efficiency, $k=3$, correlated scenarios

\begin{tabular}{|c|c|c|c|c|c|c|c|c|c|c|c|c|}
\hline & \multirow[b]{2}{*}{$|V|$} & \multirow[b]{2}{*}{$|E|$} & \multirow[b]{2}{*}{$r$} & \multicolumn{3}{|c|}{ EL-Highly } & \multicolumn{3}{|c|}{$\underline{\text { RL-Highly }}$} & \multicolumn{3}{|l|}{ sols } \\
\hline & & & & $\min$ & $\max$ & avg & $\min$ & $\max$ & avg & $\min$ & $\max$ & avg \\
\hline 2 & 101 & 446.90 & 2 & 0.04 & 0.64 & 0.32 & 0.03 & 0.53 & 0.26 & 19 & 125 & 58.40 \\
\hline 6 & 101 & 450.50 & 4 & 0.27 & 2.06 & 0.69 & 0.26 & 1.15 & 0.58 & 23 & 208 & 70.10 \\
\hline 10 & 101 & 445.50 & 6 & 0.16 & 2.71 & 1.11 & 0.23 & 2.97 & 1.21 & 19 & 230 & 102.00 \\
\hline 14 & 101 & 447.00 & 8 & 0.27 & 2.21 & 0.86 & 0.35 & 2.30 & 1.02 & 15 & 130 & 62.70 \\
\hline 18 & 201 & 913.20 & 2 & 0.36 & 2.78 & 1.39 & 0.32 & 2.10 & 1.09 & 40 & 212 & 117.60 \\
\hline 22 & 201 & 892.60 & 4 & 0.36 & 4.78 & 1.98 & 0.42 & 4.08 & 1.94 & 54 & 205 & 103.30 \\
\hline 26 & 201 & 906.70 & 6 & 0.72 & 6.30 & 2.54 & 0.89 & 5.77 & 2.59 & 8 & 163 & 68.30 \\
\hline 30 & 201 & 908.70 & 8 & 0.55 & 11.45 & 4.23 & 0.64 & 8.89 & 3.88 & 32 & 219 & 81.00 \\
\hline 34 & 401 & 1818.60 & 2 & 1.90 & 9.18 & 4.44 & 1.32 & 7.09 & 3.32 & 19 & 220 & 117.60 \\
\hline 38 & 401 & 1781.60 & 4 & 1.96 & 18.64 & 8.95 & 1.95 & 17.04 & 8.28 & 11 & 248 & 101.90 \\
\hline 42 & 401 & 1784.90 & 6 & 4.68 & 17.15 & 9.49 & 4.11 & 15.19 & 8.57 & 16 & 215 & 108.30 \\
\hline 46 & 401 & 1813.50 & 8 & 2.96 & 47.23 & 17.73 & 3.53 & 38.51 & 17.07 & 22 & 122 & 66.90 \\
\hline 50 & 801 & 3615.00 & 2 & 5.54 & 74.58 & 22.21 & 5.08 & 52.30 & 17.42 & 5 & 352 & 92.80 \\
\hline 54 & 801 & 3590.20 & 4 & 14.53 & 58.07 & 33.23 & 13.54 & 59.83 & 34.42 & 34 & 271 & 149.70 \\
\hline 58 & 801 & 3611.40 & 6 & 22.18 & 77.03 & 40.31 & 25.02 & 86.67 & 48.86 & 54 & 347 & 150.50 \\
\hline 62 & 801 & 3604.00 & 8 & 21.40 & 117.46 & 64.12 & 30.24 & 159.05 & 89.44 & 8 & 222 & 129.20 \\
\hline 66 & 1201 & 5399.90 & 2 & 14.94 & 83.02 & 37.02 & 14.74 & 60.38 & 33.47 & 31 & 285 & 165.30 \\
\hline 70 & 1201 & 5390.20 & 4 & 26.12 & 303.98 & 100.14 & 36.24 & 364.93 & 125.59 & 37 & 448 & 219.90 \\
\hline 74 & 1201 & 5422.00 & 6 & 44.32 & 242.01 & 110.85 & 59.54 & 288.50 & 140.93 & 49 & 615 & 180.40 \\
\hline 78 & 1201 & 5406.00 & 8 & 42.21 & 282.48 & 146.08 & 55.20 & 391.82 & 193.26 & 34 & 582 & 230.90 \\
\hline
\end{tabular}

https://doi.org/10.1002/net.21815 
RAITH ET AL.

T A B L E A21 NetMaker instances, flimsily robust efficiency, $k=2$, random scenarios

\begin{tabular}{|c|c|c|c|c|c|c|c|c|c|c|c|c|}
\hline & \multirow[b]{2}{*}{$|V|$} & \multirow[b]{2}{*}{$|E|$} & \multirow[b]{2}{*}{$r$} & \multicolumn{3}{|c|}{ EL-Flimsily } & \multicolumn{3}{|c|}{ RL-Flimsily } & \multicolumn{3}{|l|}{ sols } \\
\hline & & & & $\overline{\min }$ & $\max$ & $\overline{\text { avg }}$ & $\min$ & $\max$ & $\overline{\text { avg }}$ & $\overline{\min }$ & $\max$ & $\overline{\text { avg }}$ \\
\hline 3 & 101 & 444.95 & 2 & 0.00 & 0.03 & 0.01 & 0.00 & 0.01 & 0.00 & 1 & 25 & 10.05 \\
\hline 7 & 101 & 448.35 & 4 & 0.02 & 0.06 & 0.03 & 0.01 & 0.02 & 0.01 & 5 & 36 & 19.60 \\
\hline 11 & 101 & 449.35 & 6 & 0.04 & 0.14 & 0.08 & 0.01 & 0.02 & 0.01 & 9 & 56 & 26.35 \\
\hline 15 & 101 & 447.60 & 8 & 0.07 & 0.21 & 0.11 & 0.01 & 0.03 & 0.02 & 14 & 80 & 34.25 \\
\hline 19 & 201 & 908.60 & 2 & 0.02 & 0.04 & 0.03 & 0.01 & 0.02 & 0.01 & 1 & 42 & 16.65 \\
\hline 23 & 201 & 897.05 & 4 & 0.04 & 0.16 & 0.09 & 0.01 & 0.03 & 0.02 & 8 & 52 & 23.75 \\
\hline 27 & 201 & 901.05 & 6 & 0.10 & 0.33 & 0.20 & 0.02 & 0.04 & 0.03 & 10 & 74 & 35.65 \\
\hline 31 & 201 & 887.20 & 8 & 0.21 & 0.54 & 0.35 & 0.03 & 0.05 & 0.04 & 12 & 93 & 48.75 \\
\hline 35 & 401 & 1798.25 & 2 & 0.04 & 0.14 & 0.08 & 0.01 & 0.05 & 0.03 & 1 & 42 & 15.75 \\
\hline 39 & 401 & 1798.15 & 4 & 0.19 & 0.43 & 0.28 & 0.04 & 0.08 & 0.06 & 7 & 48 & 26.35 \\
\hline 43 & 401 & 1790.20 & 6 & 0.39 & 2.04 & 0.71 & 0.05 & 0.18 & 0.08 & 11 & 101 & 42.75 \\
\hline 47 & 401 & 1795.75 & 8 & 0.67 & 2.98 & 1.57 & 0.06 & 0.18 & 0.12 & 18 & 90 & 49.50 \\
\hline 51 & 801 & 3617.85 & 2 & 0.13 & 0.48 & 0.22 & 0.04 & 0.15 & 0.07 & 2 & 46 & 18.55 \\
\hline 55 & 801 & 3621.45 & 4 & 0.64 & 1.28 & 0.95 & 0.10 & 0.20 & 0.14 & 9 & 80 & 35.80 \\
\hline 59 & 801 & 3605.35 & 6 & 1.27 & 3.59 & 2.43 & 0.13 & 0.28 & 0.21 & 11 & 92 & 46.95 \\
\hline 63 & 801 & 3618.15 & 8 & 2.59 & 7.72 & 5.12 & 0.18 & 0.38 & 0.30 & 7 & 119 & 58.00 \\
\hline 67 & 1201 & 5393.00 & 2 & 0.21 & 0.59 & 0.43 & 0.07 & 0.21 & 0.15 & 4 & 39 & 19.05 \\
\hline 71 & 1201 & 5406.85 & 4 & 1.23 & 2.63 & 1.88 & 0.18 & 0.32 & 0.25 & 8 & 52 & 37.85 \\
\hline 75 & 1201 & 5394.40 & 6 & 2.79 & 9.22 & 4.83 & 0.25 & 0.59 & 0.38 & 13 & 103 & 43.20 \\
\hline 79 & 1201 & 5408.90 & 8 & 5.70 & 16.11 & 9.58 & 0.38 & 0.81 & 0.53 & 35 & 154 & 80.60 \\
\hline 83 & 1601 & 7188.15 & 2 & 0.43 & 1.06 & 0.66 & 0.12 & 0.25 & 0.17 & 4 & 36 & 17.15 \\
\hline 87 & 1601 & 7240.00 & 4 & 1.88 & 3.68 & 2.59 & 0.27 & 0.49 & 0.35 & 8 & 86 & 41.85 \\
\hline 91 & 1601 & 7196.85 & 6 & 4.15 & 10.78 & 7.25 & 0.38 & 0.76 & 0.55 & 15 & 93 & 54.30 \\
\hline 95 & 1601 & 7184.00 & 8 & 7.01 & 19.52 & 13.43 & 0.51 & 1.09 & 0.81 & 23 & 156 & 67.90 \\
\hline
\end{tabular}

T A B L E A22 NetMaker instances, flimsily robust efficiency, $k=3$, random scenarios

\begin{tabular}{|c|c|c|c|c|c|c|c|c|c|c|c|c|}
\hline & & & & EL-Flin & & & RL-F & & & sols & & \\
\hline & $|V|$ & $|E|$ & $r$ & $\min$ & $\max$ & avg & $\overline{\min }$ & $\max$ & avg & $\overline{\min }$ & $\max$ & avg \\
\hline 4 & 101 & 453.40 & 2 & 0.18 & 1.46 & 0.74 & 0.07 & 0.52 & 0.28 & 49 & 304 & 193.10 \\
\hline 8 & 101 & 449.40 & 4 & 0.48 & 18.63 & 4.08 & 0.14 & 2.74 & 0.73 & 71 & 417 & 250.40 \\
\hline 12 & 101 & 437.10 & 6 & 1.41 & 7.05 & 3.15 & 0.26 & 1.04 & 0.51 & 157 & 408 & 253.20 \\
\hline 16 & 101 & 453.90 & 8 & 4.77 & 61.39 & 16.52 & 0.68 & 4.08 & 1.50 & 220 & 1613 & 582.60 \\
\hline 20 & 201 & 900.30 & 2 & 0.51 & 6.29 & 2.88 & 0.23 & 1.53 & 0.88 & 92 & 400 & 231.50 \\
\hline 24 & 201 & 896.10 & 4 & 3.73 & 21.87 & 10.32 & 0.84 & 3.77 & 1.87 & 77 & 725 & 295.50 \\
\hline 28 & 201 & 896.50 & 6 & 7.29 & 51.11 & 24.63 & 1.15 & 5.11 & 2.67 & 97 & 647 & 387.70 \\
\hline 32 & 201 & 903.70 & 8 & 11.55 & 134.84 & 63.47 & 1.18 & 9.03 & 4.79 & 63 & 1065 & 504.50 \\
\hline 36 & 401 & 1805.20 & 2 & 5.70 & 38.00 & 13.38 & 1.85 & 9.47 & 3.74 & 156 & 619 & 342.60 \\
\hline 40 & 401 & 1793.40 & 4 & 22.90 & 108.80 & 55.99 & 3.63 & 16.55 & 9.02 & 79 & 783 & 318.70 \\
\hline 44 & 401 & 1817.20 & 6 & 72.33 & 408.14 & 159.84 & 8.27 & 22.36 & 13.83 & 64 & 1598 & 545.00 \\
\hline 48 & 401 & 1797.20 & 8 & 105.67 & 430.15 & 224.25 & 8.51 & 33.47 & 17.70 & 140 & 1727 & 687.20 \\
\hline 52 & 801 & 3597.50 & 2 & 30.10 & 70.57 & 56.05 & 9.89 & 24.40 & 18.64 & 82 & 791 & 362.80 \\
\hline 56 & 801 & 3589.20 & 4 & 94.42 & 491.09 & 189.64 & 16.24 & 79.86 & 33.32 & 66 & 832 & 517.30 \\
\hline 60 & 801 & 3571.90 & 6 & 164.83 & 803.24 & 460.76 & 20.61 & 91.63 & 49.34 & 265 & 1509 & 605.50 \\
\hline 64 & 801 & 3608.80 & 8 & 402.48 & 1564.07 & 719.01 & 31.57 & 107.47 & 58.93 & 310 & 3194 & 851.30 \\
\hline 68 & 1201 & 5415.70 & 2 & 47.09 & 146.78 & 83.11 & 16.56 & 50.89 & 31.11 & 148 & 1050 & 471.60 \\
\hline 72 & 1201 & 5384.90 & 4 & 168.73 & 614.03 & 364.31 & 32.15 & 103.32 & 70.07 & 212 & 686 & 402.00 \\
\hline 76 & 1201 & 5398.90 & 6 & 514.27 & 2618.41 & 1192.11 & 66.12 & 270.39 & 138.81 & 420 & 2117 & 993.50 \\
\hline 80 & 1201 & 5393.00 & 8 & 1136.37 & 3071.61 & 1779.11 & 112.17 & 288.15 & 185.88 & 280 & 3802 & 1171.20 \\
\hline
\end{tabular}

https://doi.org/10.1002/net. 21815 
A.2. Extensions of Labeling Algorithms

${ }^{44} \perp$ WILEY

RAITH ET AL.

T A B L E A23 NetMaker instances, highly robust efficiency, $k=2$, random scenarios

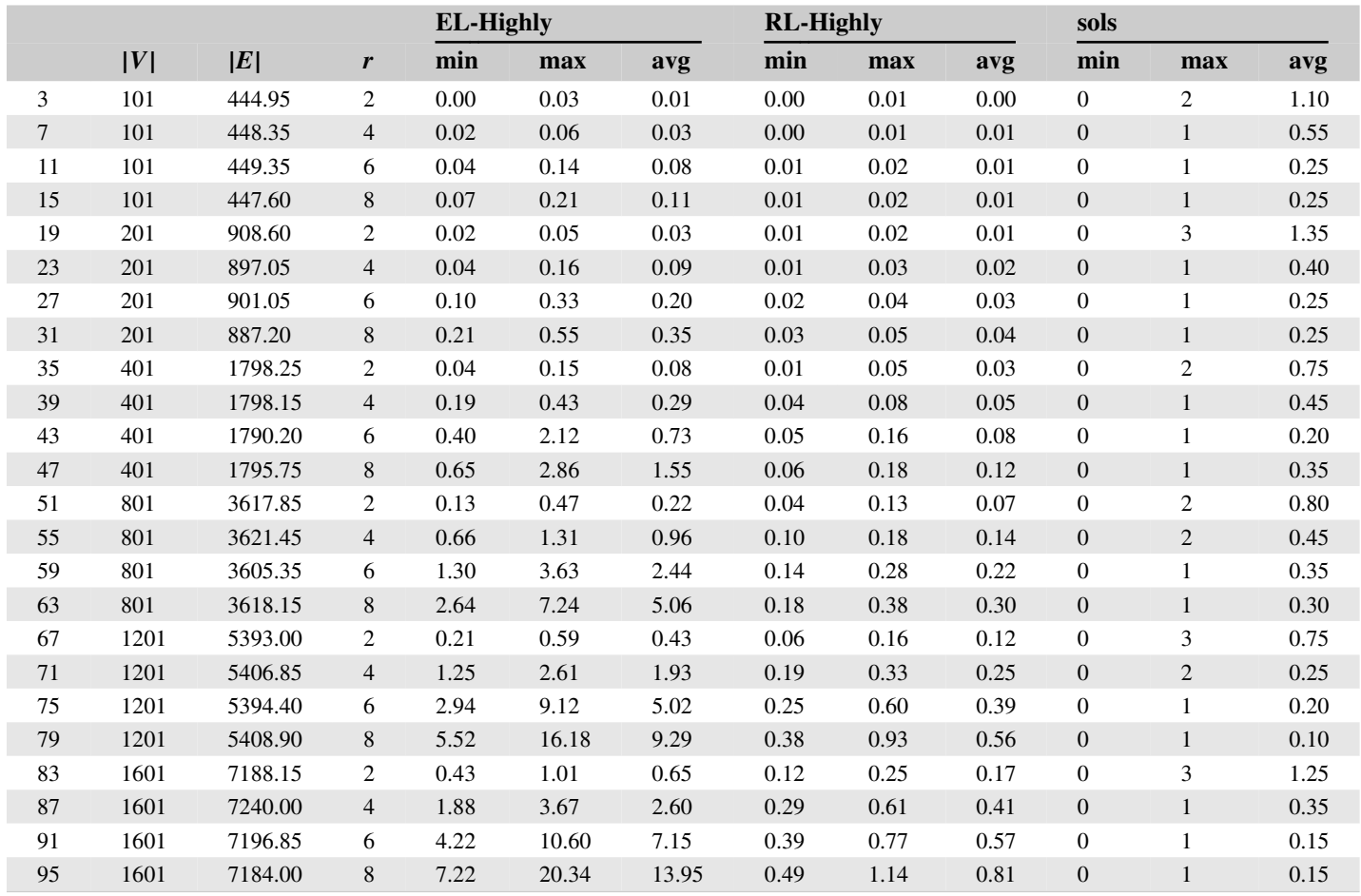

T A B L E A24 NetMaker instances, highly robust efficiency, $k=3$, random scenarios

\begin{tabular}{|c|c|c|c|c|c|c|c|c|c|c|c|c|}
\hline & \multirow[b]{2}{*}{$|V|$} & \multirow[b]{2}{*}{$|E|$} & \multirow[b]{2}{*}{$r$} & \multicolumn{3}{|c|}{ EL-Highly } & \multicolumn{3}{|c|}{ RL-Highly } & \multicolumn{3}{|l|}{ sols } \\
\hline & & & & $\overline{\min }$ & $\max$ & avg & $\overline{\min }$ & $\max$ & avg & $\min$ & $\max$ & avg \\
\hline 4 & 101 & 453.40 & 2 & 0.19 & 1.53 & 0.75 & 0.07 & 0.52 & 0.28 & 12 & 71 & 35.90 \\
\hline 8 & 101 & 449.40 & 4 & 0.50 & 19.02 & 4.19 & 0.14 & 2.77 & 0.74 & 6 & 25 & 12.80 \\
\hline 12 & 101 & 437.10 & 6 & 1.43 & 7.75 & 3.26 & 0.26 & 1.00 & 0.51 & 4 & 13 & 7.90 \\
\hline 16 & 101 & 453.90 & 8 & 4.93 & 62.67 & 16.76 & 0.69 & 4.16 & 1.54 & 3 & 15 & 8.30 \\
\hline 20 & 201 & 900.30 & 2 & 0.54 & 6.69 & 3.02 & 0.22 & 1.54 & 0.87 & 19 & 94 & 44.90 \\
\hline 24 & 201 & 896.10 & 4 & 3.89 & 21.42 & 10.25 & 0.83 & 3.80 & 1.86 & 5 & 40 & 16.80 \\
\hline 28 & 201 & 896.50 & 6 & 7.23 & 48.70 & 25.10 & 1.15 & 4.90 & 2.61 & 2 & 30 & 10.20 \\
\hline 32 & 201 & 903.70 & 8 & 13.03 & 142.01 & 66.90 & 1.16 & 8.94 & 4.86 & 3 & 12 & 6.50 \\
\hline 36 & 401 & 1805.20 & 2 & 5.58 & 41.46 & 13.69 & 1.85 & 9.56 & 3.74 & 20 & 107 & 53.10 \\
\hline 40 & 401 & 1793.40 & 4 & 23.04 & 101.53 & 57.26 & 3.67 & 16.09 & 8.83 & 4 & 28 & 16.90 \\
\hline 44 & 401 & 1817.20 & 6 & 77.99 & 432.07 & 170.98 & 8.89 & 22.13 & 13.68 & 1 & 29 & 12.70 \\
\hline 48 & 401 & 1797.20 & 8 & 104.31 & 449.94 & 233.68 & 8.56 & 30.01 & 17.05 & 0 & 23 & 7.60 \\
\hline 52 & 801 & 3597.50 & 2 & 30.75 & 77.63 & 63.80 & 9.93 & 24.13 & 18.69 & 25 & 141 & 61.80 \\
\hline 56 & 801 & 3589.20 & 4 & 84.78 & 419.81 & 174.53 & 16.97 & 80.77 & 33.61 & 4 & 41 & 21.10 \\
\hline 60 & 801 & 3571.90 & 6 & 166.80 & 782.04 & 429.02 & 20.46 & 90.55 & 48.08 & 3 & 23 & 10.90 \\
\hline 64 & 801 & 3608.80 & 8 & 351.56 & 1467.07 & 659.68 & 31.51 & 107.18 & 58.12 & 4 & 19 & 8.40 \\
\hline 68 & 1201 & 5415.70 & 2 & 50.67 & 149.00 & 85.00 & 16.40 & 48.45 & 30.19 & 17 & 168 & 60.10 \\
\hline 72 & 1201 & 5384.90 & 4 & 174.05 & 617.25 & 365.62 & 32.65 & 102.50 & 69.82 & 3 & 58 & 17.40 \\
\hline 76 & 1201 & 5398.90 & 6 & 493.57 & 2448.50 & 1218.99 & 64.34 & 270.00 & 135.88 & 5 & 25 & 15.00 \\
\hline 80 & 1201 & 5393.00 & 8 & 1216.22 & 3391.55 & 1895.47 & 103.63 & 267.30 & 175.94 & 2 & 17 & 9.90 \\
\hline
\end{tabular}

https://doi.org/10.1002/net.21815 


\section{A.3. Min-Ordering and Max-Ordering Scalarization Methods for Multi-Objective Robust Optimization}

submitted to the European Journal of Operational Research in January 2018 and available as preprint (Schmidt et al., 2018)

Authors: Marie Schmidt, Anita Schöbel, Lisa Thom 


\title{
Min-ordering and max-ordering scalarization methods for multi-objective robust optimization
}

\author{
Marie Schmidt ${ }^{\mathrm{a}}$, Anita Schöbel ${ }^{\mathrm{b}}$, and Lisa Thom ${ }^{\mathrm{b}, *}$

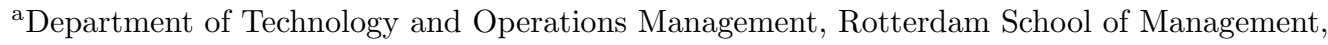 \\ Erasmus University Rotterdam, postal address: PO Box 1738, 3000 DR Rotterdam, The \\ Netherlands, email address: schmidt2@rsm.nl \\ ${ }^{b}$ Institut für Numerische und Angewandte Mathematik, Universität Göttingen, postal address: \\ Lotzestr. 16-18, 37083 Göttingen, Germany, email addresses: schoebel@math.uni-goettingen.de \\ (Anita Schöbel), l.thom@math.uni-goettingen.de (Lisa Thom) \\ ${ }^{*}$ Corresponding author, email address: l.thom@math.uni-goettingen.de
}

\begin{abstract}
Several robustness concepts for multi-objective uncertain optimization have been developed during the last years, but not many solution methods. In this paper we introduce two methods to find minmax robust efficient solutions based on scalarizations: the minordering and the max-ordering method. We show that all point-based minmax robust weakly efficient solutions can be found with the max-ordering method and that the minordering method finds set-based minmax robust weakly efficient solutions, some of which cannot be found with formerly developed scalarization based methods. We then show how the scalarized problems may be approached for multi-objective uncertain combinatorial optimization problems with special uncertainty sets. We develop compact mixed-integer linear programming formulations for multi-objective extensions of bounded uncertainty. For interval uncertainty, we show that the resulting problems reduce to well-known singleobjective problems.
\end{abstract}

\section{Introduction}

When applying optimization techniques to real-world problems, one often encounters the difficulties, that several objectives need to be optimized at the same time and that not all parameters are known exactly in advance. In multi-objective optimization several objectives are optimized simultaneously by choosing a (Pareto) efficient solution that cannot be improved in one objective without worsening it in another objective. Robust optimization is a way to handle uncertainties, without having to assume any information on probability distributions, hedging against (all) possible outcomes. During the last years, concepts of those fields have been combined to multi-objective robust optimization.

Several concepts on how to define robust solutions in multi-objective optimization have been developed. The common (single-objective) concept of minmax robustness aims to find a solution that minimizes the objective function in the worst case. One generalization to multi-objective optimization, which we call point-based minmax robust efficiency, was first introduced by [KL12]. They consider the worst case in each objective independently, which results in a deterministic multi-objective problem with bottleneck objective functions, called 
the robust counterpart. However, the resulting worst case point for a solution can differ significantly from the possible outcomes. Therefore, a second generalization of minmax robustness for multiple objectives has been developed by [EIS14]. They look at the outcome set of a solution under every scenario and compare these sets to each other to find so-called set-based minmax robust efficient solutions. A comparison of these two and other concepts for robust efficiency can be found in [IS16] and [WD16].

Common methods to find efficient solutions in the deterministic case, i.e. without uncertainty, are so-called scalarization methods, where the multi-objective problem is transformed to a family of single-objective problems, whose solutions are (weakly) efficient for the original problem. By solving the resulting problems, several different (and possibly all) efficient solutions are found. For an overview on scalarization methods see, e.g., [Ehr06].

In the uncertain case, several methods to find minmax robust efficient solutions have been developed, which are based on scalarizations: on the weighted sum and $\epsilon$-constraint scalarization ([EIS14]), on the augmented weighted Chebyshev scalarization ([Ide14]) and on pnorm scalarizations $([\mathrm{BF} 17])$. Point-based minmax robust efficient solutions can also be found by applying deterministic scalarization methods to the robust counterpart (see, e.g., [HNS13, KL12, FW14]).

In this paper we introduce two new methods to find minmax robust efficient solutions based on scalarizations: the max-ordering and min-ordering method, resulting in problems of the form min-max-max respective min-max-min. The min-ordering problem can therefore be interpreted as a so-called adjustable robust problem [BTGGN04], where only part of the decisions has to be made before the realization of the uncertain parameters.

In robust optimization, the considered uncertainty set, i.e., the possible values the uncertain parameters can attain, plays an important role w.r.t. solvability and complexity of the resulting robust problems. In this paper we investigate the min-ordering and max-ordering optimization problems for multi-objective minmax robust combinatorial optimization problems with specific uncertainty sets: One popular assumption is that each parameter attains a value in a given interval independently of the realization of the other parameters (interval uncertainty). Based on this, [BS03] introduced the (single-objective) concept of bounded uncertainty, assuming that the parameters vary in intervals, but the worst case is not attained for all parameters simultaneously. Uncertainty sets for multi-objective optimization based on bounded uncertainty have been considered in [DKW12, $\left.\mathrm{WLD}^{+} 17\right]$ (only considering uncertainty in the constraints) and [HNS13, RSST18] (resulting in an objective-wise uncertainty set). We introduce an extension of bounded uncertainty to multi-objective optimization for the case that the uncertainties in the objectives are not independent of each other.

Solution approaches for multi-objective minmax robust combinatorial problems with objectivewise bounded uncertainty have been developed in [RSST18]. [KRSS16] consider bi-objective robust combinatorial problems with finite and polyhedral uncertainty sets for several robustness concepts. The multi-objective robust version of the shortest path problem with finite uncertainty set is considered in [RSST17], where labeling algorithms are extended in order to find robust efficient solutions.

This paper is structured as follows: First, we give a short introduction to multi-objective robust optimization. In Section 3 we introduce the min-ordering and max-ordering optimization problems and show their general properties. In Section 4 we consider combinatorial multi-objective optimization problems with particular uncertainty sets and investigate the complexity and solvability of the resulting min-ordering and max-ordering problems. 


\section{Preliminaries}

In this section we introduce some general notation and give a short introduction to multiobjective optimization and multi-objective robust optimization.

Throughout this paper, we use the symbols $<$ (strictly less than) and $\leqq$ (less than or equal to) to compare values in $\mathbb{R}$. Further, $\partial M$ denotes the boundary of a set $M \subseteq \mathbb{R}^{k}$ and we use $i \in[k]$ as an abbreviation for $i \in\{1, \ldots, k\}$.

To shorten the text we use a [./.] notation, e.g., instead of " $x$ is smaller than $y$ if $x<y$ and $x$ is smaller than or equal to $y$ if $x \leqq y$ " we write " $x$ is smaller than $[\cdot /$ or equal to] $y$ if $x[</ \leqq] y^{\prime \prime}$.

\subsection{Multi-objective robust optimization}

Definition 1. Given a set $\mathcal{X}$ of feasible solutions and $k \in \mathbb{N}$ objective functions $z_{1}, \ldots, z_{k}$ : $\mathcal{X} \rightarrow \mathbb{R}$, we call

$$
\min _{x \in \mathcal{X}} z(x)=\left(\begin{array}{c}
z_{1}(x) \\
\vdots \\
z_{k}(x)
\end{array}\right)
$$

$a$ multi-objective optimization problem (MOP).

If $k=1$ we say that the problem is a single-objective problem. For $k \geqq 2$, a solution that minimizes all objectives simultaneously does usually not exist. Therefore, we use the concept of efficient solutions.

Definition 2. For two vectors $y^{1}, y^{2} \in \mathbb{R}^{k}$ we use the notation

$$
\begin{aligned}
& y^{1} \leq y^{2} \Leftrightarrow y_{i}^{1}<y_{i}^{2} \text { for } i \in[k], \\
& y^{1} \leq y^{2} \Leftrightarrow y_{i}^{1} \leqq y_{i}^{2} \text { for } i \in[k] \text { and } y^{1} \neq y^{2}, \\
& y^{1} \leqq y^{2} \Leftrightarrow y_{i}^{1} \leqq y_{i}^{2} \text { for } i \in[k] .
\end{aligned}
$$

We also define $\mathbb{R}_{[>/ \geq / \geqq]}^{k}:=\left\{y \in \mathbb{R}^{k}: 0[</ \leq / \leqq] y\right\}$.

Definition 3. A solution $x \in \mathcal{X}$ is a [weakly/./strictly] efficient solution for (MOP), if there is no $x^{\prime} \in \mathcal{X}$ such that $z\left(x^{\prime}\right)[</ \leq / \leqq] z(x)$.

Note that a solution $x \in \mathcal{X}$ is [weakly/./strictly] efficient if and only if there is no $x^{\prime} \in X$ with

$$
z\left(x^{\prime}\right) \in z(x)-\left(\mathbb{R}_{[>/ \geq / \geqq]}^{k}\right) .
$$

We now assume that the input data is uncertain, i.e., not all parameters are exactly known in advance. Instead, they depend on a scenario, which will only be revealed after one has chosen a solution. The set $\mathcal{U}$ of all possible scenarios is called the uncertainty set.

Definition 4. Given a feasible set of solutions $\mathcal{X}$, an uncertainty set $\mathcal{U}$, and a multi-objective function $z: \mathcal{X} \times \mathcal{U} \rightarrow \mathbb{R}^{k}$, the family of multi-objective optimization problems

$$
\left(\min _{x \in \mathcal{X}} z(x, \xi), \xi \in \mathcal{U}\right)
$$

is called a multi-objective uncertain optimization problem (MOUP). 
In the following we assume $\mathcal{X}$ and $\mathcal{U}$ to be compact and non-empty and the $z_{i}$ to be continuous in $x$ and $\xi$. If a problem or part of a problem is not subject to uncertainty, we say that it is deterministic, e.g., this is the case for a (MOUP) with $|\mathcal{U}|=1$.

Note that the formulation in Definition 4 only considers uncertainty in the objective function. If the constraints, i.e., the set of feasible solutions, are subject to uncertainty, we aim to find solutions which are feasible in all scenarios (as proposed in the seminal works on robustness, see, e.g., [Soy73, BTN98]). For this purpose, the sets of feasible solutions under all scenarios can be intersected in advance to obtain a (deterministic) set of robust feasible solutions. Hence, in the following, we assume the feasible set $\mathcal{X}$ to be deterministic.

To decide what is a good solution for a multi-objective uncertain problem is not trivial. In single-objective robust optimization one looks for so-called robust optimal solutions. Often these are defined as solutions, which have a minimal worst case value, i.e., one solves $\min _{x \in \mathcal{X}} \max _{\xi \in \mathcal{U}} z(x, \xi)$ (see, e.g., [BTEGN09]). This concept has been generalized to robust efficiency for multi-objective problems in various ways (e.g., [KL12, EIS14]), since the notion of worst case is not clear in the multi-objective case.

We present the two most common concepts for minmax robust efficiency: point-based minmax robust efficiency and set-based minmax robust efficiency. For point-based minmax robust efficiency, we determine the worst case for each solution $x$ and objective $i$ individually, and compare the solutions w.r.t. the resulting point $\bar{z}(x)$. For set-based minmax robust efficiency, we check whether there exists a solution $\xi \in \mathcal{U}$ with $\{z(x, \xi): \xi \in \mathcal{U}\} \subseteq\{z(x, \xi): \xi \in \mathcal{U}\}-\mathbb{R}_{\geq}^{k}$ (analogous to determining efficiency in the deterministic case by checking whether a solution $x^{\prime} \in \mathcal{X}$ with $z\left(x^{\prime}\right) \in z(x)-\mathbb{R}_{\geq}^{k}$ exists).

Definition 5 ([KL12], [EIS14] ). Given a multi-objective uncertain optimization problem, we define

$$
\bar{z}(x):=\left(\begin{array}{c}
\max _{\xi \in \mathcal{U}} z_{1}(x, \xi) \\
\vdots \\
\max _{\xi \in \mathcal{U}} z_{k}(x, \xi)
\end{array}\right)
$$

A solution $x \in \mathcal{X}$ is point-based minmax robust [weakly $/ \cdot /$ strictly] efficient for (MOUP) (abbreviated: pointMR [weakly/./strictly] efficient), if it is a [weakly/./strictly] efficient solution for the robust counterpart $\min _{x \in \mathcal{X}} \bar{z}(x)$, i.e., if there is no $x^{\prime} \in \mathcal{X}$ with

$$
\bar{z}\left(x^{\prime}\right) \in \bar{z}(x)-\mathbb{R}_{[>/ \geq / \geqq]}^{k} .
$$

Defining

$$
z_{\mathcal{U}}(x):=\{z(x, \xi): \xi \in \mathcal{U}\}
$$

a solution $x \in \mathcal{X}$ is set-based minmax robust [weakly $/ \cdot /$ strictly] efficient for (MOUP) (abbreviated: setMR [weakly/./strictly] efficient), if there exists no $x^{\prime} \in \mathcal{X}$ with

$$
z_{\mathcal{U}}\left(x^{\prime}\right) \subseteq z_{\mathcal{U}}(x)-\mathbb{R}_{[>/ \geq / \geqq]}^{k}
$$

Both concepts reduce to minmax robustness for $k=1$, i.e., the pointMR efficient solutions and setMR efficient solutions are then identical to the solutions of $\min _{x \in \mathcal{X}} \max _{\xi \in \mathcal{U}} z_{1}(x, \xi)$. Note that every pointMR [weakly/strictly] efficient solution is also setMR [weakly/strictly] efficient and that the two concepts coincide, if (MOUP) is objective-wise uncertain, i.e., if $\mathcal{U}=\mathcal{U}_{1} \times \ldots \times \mathcal{U}_{k}$ and $z_{i}(x, \xi)=z_{i}\left(x, \xi_{i}\right), \xi_{i} \in \mathcal{U}_{i} \forall i \in[k]$. 


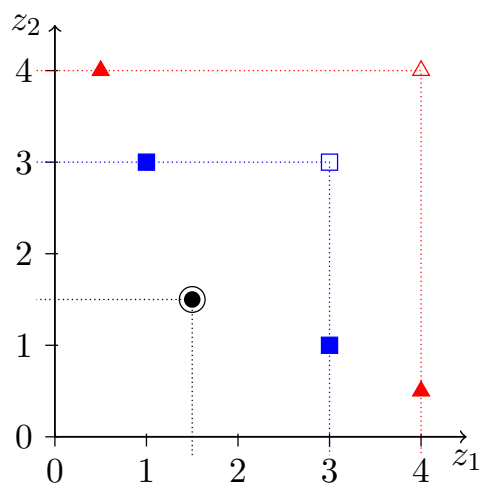

(a) Only $x^{1}$ is pointMR efficient.

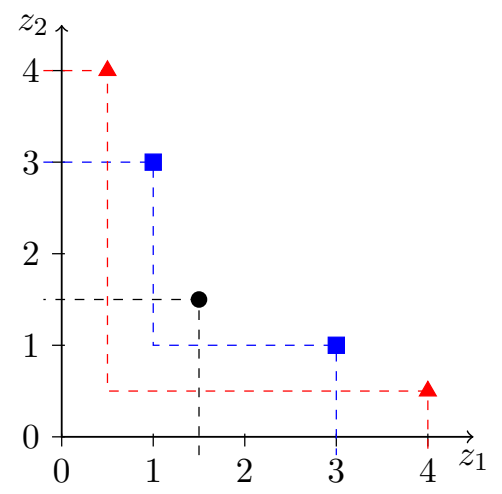

- $z_{\mathcal{U}}\left(x^{1}\right)$

- $z_{\mathcal{U}}\left(x^{2}\right)$

- $z_{\mathcal{U}}\left(x^{3}\right)$

$\bar{z}\left(x^{1}\right)$

$\triangle \bar{z}\left(x^{2}\right)$

$\square \bar{z}\left(x^{3}\right)$

$\ldots \ldots \ldots \ldots . . . \partial\left(\bar{z}(x)-\mathbb{R}_{\geqq}^{2}\right)$

- - $\partial\left(z \mathcal{U}(x)-\mathbb{R}_{\geqq}^{2}\right)$

(b) All solutions are setMR efficient.

Figure 1: Determining pointMR efficient solutions and setMR efficient solutions for the instance in Example 6.

Example 6. Let a multi-objective uncertain optimization problem be given with $\mathcal{X}:=\left\{x^{1}, x^{2}, x^{3}\right\}$, $\mathcal{U}:=\left\{\xi^{1}, \xi^{2}\right\}$ and

$$
\begin{aligned}
& z\left(x^{1}, \xi^{1}\right)=z\left(x^{1}, \xi^{2}\right)=(1.5,1.5) \\
& z\left(x^{2}, \xi^{1}\right)=(0.5,4), z\left(x^{2}, \xi^{2}\right)=(4,0.5) \\
& z\left(x^{3}, \xi^{1}\right)=(1,3), z\left(x^{3}, \xi^{2}\right)=(3,1) .
\end{aligned}
$$

Figure $1(a)$ shows $\bar{z}(x)$ and $\partial\left(\bar{z}(x)-\mathbb{R}_{\geq}^{k}\right)$ and Figure $1(b)$ shows $z_{\mathcal{U}}(x)$ and $\partial\left(z_{\mathcal{U}}(x)-\mathbb{R}_{\geq}^{k}\right)$ for $x \in \mathcal{X}$. All three solutions are setMR efficient, whereas only $x^{1}$ is pointMR efficient.

The following lemma characterizes setMR efficient solutions.

Lemma 7 ([EIS14]). Given a multi-objective uncertain optimization problem (MOUP). For all $x, x^{\prime} \in \mathcal{X}$,

$$
z_{\mathcal{U}}\left(x^{\prime}\right) \subseteq z_{\mathcal{U}}(x)-\mathbb{R}_{[>/ \geq / \geqq]}^{k} \Leftrightarrow \forall \xi \in \mathcal{U} \exists \eta \in \mathcal{U}: z\left(x^{\prime}, \xi\right)[</ \leq / \leqq] z(x, \eta)
$$

\subsection{Methods to find robust efficient solutions based on scalarizations}

In (deterministic) multi-objective optimization it is common to find a set of efficient solutions with a scalarization method, i.e., by solving a family of single-objective, so-called scalarized, problems (see, e.g., [Ehr06]). For finding pointMR efficient solutions, these methods can directly be applied to the robust counterpart $\min _{x \in \mathcal{X}} \bar{z}(x)$. In case of set-based minmax robust efficiency, the extension of scalarization methods is not as straightforward, because the robust counterpart is a set-valued problem. The following methods to find setMR efficient solutions based on scalarizations have been developed.

[EIS14] introduce two methods based on scalarizations: The weighted sum scalarization method and the $\epsilon$-constraint method, which are extensions of the corresponding methods for the deterministic case. They show that both methods find setMR weakly efficient solutions. The solutions for the weighted sum scalarized problems are even setMR efficient, if 
the weights are chosen strictly greater than zero. The solutions found with the $\epsilon$-constraint method are always pointMR weakly efficient. The authors show that the two methods do not always find the same solutions and that there can exist setMR efficient solutions, which cannot be found by either of these methods.

[Ide14] introduce a method based on the (augmented) weighted Chebyshev scalarization with reference point 0. [Ide14] show that all solutions found with this (augmented) weighted Chebyshev method are setMR weakly efficient. In case of objective-wise uncertainty, the scalarized problem in [Ide14] is identical to the scalarized problem in [HNS13] (if the robust utopian point in [HNS13] can be chosen as 0), where the deterministic augmented weighted Chebychev method is applied to the robust counterpart $\min _{x \in \mathcal{X}} \bar{z}(x)$ to find pointMR efficient solutions. [BF17] consider order-preserving scalarizing functions $s: \mathbb{R}^{k} \rightarrow \mathbb{R}$ and the resulting scalarized problems $\min _{x \in \mathcal{X}} \max _{\xi \in \mathcal{U}} s(z(x))$. They show that for so-called strongly increasing scalarizing functions the solutions for the scalarized problem are setMR efficient. In an application they consider weighted $p$-norms as scalarizing functions, resulting in the $p$-norm scalarization method (e.g., the weighted sum scalarization method for $p=1$ ).

\section{Min-ordering and max-ordering method for multi-objective uncertain problems}

Definition 8. Let

$$
(P)\left(\min _{x \in \mathcal{X}} z(x, \xi), \xi \in \mathcal{U}\right)
$$

be a multi-objective uncertain optimization problem. For a given weight vector $\lambda \in \mathbb{R}_{>}^{k}$ and reference point $r \in \mathbb{R}^{k}$ we define the corresponding min-ordering optimization problem as

$$
(P-\min (r, \lambda)) \min _{x \in \mathcal{X}} \max _{\xi \in \mathcal{U}} \min _{i \in[k]} \lambda_{i}\left(z_{i}(x, \xi)-r_{i}\right)
$$

and the corresponding max-ordering optimization problem as

$$
(P-\max (r, \lambda)) \min _{x \in \mathcal{X}} \max _{\xi \in \mathcal{U}} \max _{i \in[k]} \lambda_{i}\left(z_{i}(x, \xi)-r_{i}\right) .
$$

We further denote the objective value for a given $x \in \mathcal{X}$ by

$$
\begin{aligned}
\alpha^{\min }(x, r, \lambda) & :=\max _{\xi \in \mathcal{U}} \min _{i \in[k]} \lambda_{i}\left(z_{i}(x, \xi)-r_{i}\right) & & \text { for }(P-\min (r, \lambda)), \\
\alpha^{\max }(x, r, \lambda) & :=\max _{\xi \in \mathcal{U}} \max _{i \in[k]} \lambda_{i}\left(z_{i}(x, \xi)-r_{i}\right) & & \text { for }(P-\max (r, \lambda)) .
\end{aligned}
$$

Note that $\alpha^{\min }(x, r, \lambda)$ and $\alpha^{\max }(x, r, \lambda)$ exist for all $x \in \mathcal{X}$ because $\mathcal{U}$ is compact and nonempty and the finitely many functions $z_{i}(x, \cdot): \mathcal{U} \rightarrow \mathbb{R}$ are continuous. The values $\alpha^{\min }(x, r, \lambda)$ and $\alpha^{\max }(x, r, \lambda)$ also have a geometric interpretation, which we detail in Section 3.1 .

In Sections 3.2 and 3.3, we show that optimal solutions for $(\mathrm{P}-\min (r, \lambda))$ and $(\mathrm{P}-\max (r, \lambda))$ are setMR weakly efficient and solutions for $(\mathrm{P}-\max (r, \lambda))$ even pointMR weakly efficient. Similar to the existing methods discussed in Section 2.2, we obtain a min-ordering resp. max-ordering scalarization method to find a set of robust efficient solutions by varying the 
parameters $r, \lambda$ and solving the resulting problems $(\mathrm{P}-\min (r, \lambda)) \operatorname{resp} . \quad(\mathrm{P}-\max (r, \lambda))$. The max-ordering scalarization method is similar to the weighted Chebyshev method for multiobjective robust problems given in [Ide14] (and for objective-wise uncertainty in [HNS13]), but with arbitrary reference point.

Before investigating properties of the solutions for $(\mathrm{P}-\min (r, \lambda))$ and $(\mathrm{P}-\max (r, \lambda))$, we provide a brief example to give an intuition on their meaning for the original problem:

Consider a student organization who wants to offer cheap lunch for students in several university towns and has to decide on a dish $x \in \mathcal{X}$ in advance. They can price the dish differently in each town and because of a very small profit margin the price depends on the prices of the ingredients in the supermarket in town. They aim to minimize the lunch prices in all towns simultaneously, i.e., $z_{i}(x, \xi)$ is the price of dish $x$ in town $i$, where the uncertainty in the price development is modeled by $\xi \in \mathcal{U}$. Solving $(\mathrm{P}-\max (r, \lambda))$ with $r=(0, \ldots, 0)^{T}, \lambda=(1, \ldots, 1)^{T}$ means then to minimize the highest price any student in any town has to pay for their meal in the worst case. Solving $(\mathrm{P}-\min (r, \lambda))$ with the same $r, \lambda$ means to minimize the best price the organization can offer in some university, assuming the worst price development. I.e., this is the price $p$ they can legitimately use in their advertisement "Cheap student lunch - starting from $p$ !", because in some town the price will not be higher than $p$.

The remainder of this section is structured as follows: We first give a geometric interpretation of the problems $(\mathrm{P}-\min (r, \lambda))$ and $(\mathrm{P}-\max (r, \lambda))$ and a characterization of their solutions in Section 3.1. We then investigate properties of the solutions found with the max-ordering method in Section 3.2 and with the min-ordering method in Section 3.3.

In Section 4 we show how $(\mathrm{P}-\min (r, \lambda))$ and $(\mathrm{P}-\max (r, \lambda))$ can be solved for multi-objective uncertain combinatorial problems with particular uncertainty sets and investigate their complexity. For this, we use the following reformulations of $(\mathrm{P}-\min (r, \lambda))$ and $(\mathrm{P}-\max (r, \lambda))$ in case of a single scenario.

Remark 9. If the uncertainty set $\mathcal{U}$ contains only one scenario $\xi$, i.e., (MOUP) is a deterministic problem, $(P$-min $(r, \lambda))$ then reduces to $\min _{x \in \mathcal{X}, i \in[k]} \lambda_{i}\left(z_{i}(x, \xi)-r_{i}\right)$. This can be solved by solving the $k$ single-objective deterministic problems

$$
\left(P_{i}\right) \min _{x \in \mathcal{X}} \lambda_{i}\left(z_{i}(x, \xi)-r_{i}\right)
$$

and choosing the best of the obtained solutions.

$(P-\max (r, \lambda))$ reduces to $\min _{x \in \mathcal{X}} \max _{i \in[k]} \lambda_{i}\left(z_{i}(x, \xi)-r_{i}\right)$, which can be interpreted as a singleobjective minmax robust problem with a discrete uncertainty set.

\subsection{Geometric interpretation of $(\mathbf{P}-\max (r, \lambda))$ and $(\mathbf{P}-\mathbf{m i n}(r, \lambda))$}

The sublevel set of the function $\max _{i \in[k]} \lambda_{i}\left(z_{i}-r_{i}\right)$ for level $\alpha \in \mathbb{R}$ is

$$
\begin{aligned}
L_{\leqq}^{\max , r, \lambda}(\alpha) & =\left\{z \in \mathbb{R}^{k}: \max _{i \in[k]} \lambda_{i}\left(z_{i}-r_{i} \leqq \alpha\right\}\right. \\
& =\left\{z \in \mathbb{R}^{k}: z_{i} \leqq \frac{\alpha}{\lambda_{i}}+r_{i} \forall i \in[k]\right\} \\
& =\left\{z \in \mathbb{R}^{k}: z \leqq \alpha\left(\frac{1}{\lambda_{1}}, \ldots, \frac{1}{\lambda_{k}}\right)^{T}+r\right\}
\end{aligned}
$$




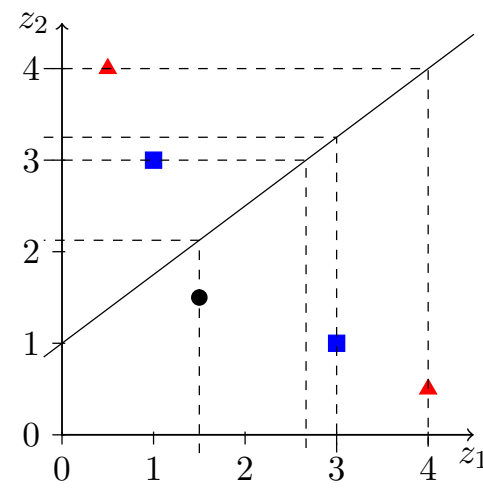

(a) $\max _{i \in[k]} \lambda_{i}\left(z_{i}-r_{i}\right)$

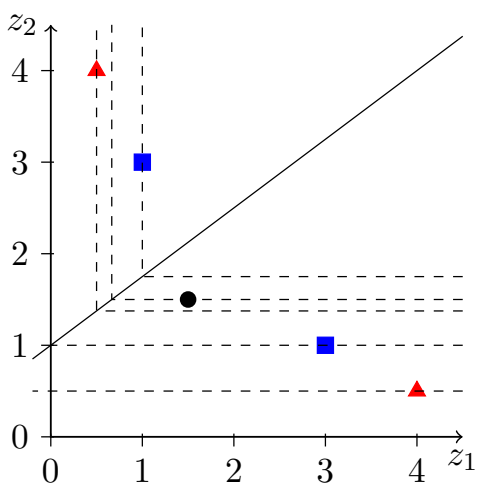

(b) $\min _{i \in[k]} \lambda_{i}\left(z_{i}-r_{i}\right)$
- $z_{\mathcal{U}}\left(x^{1}\right)$

$\Delta z_{\mathcal{U}}\left(x^{2}\right)$

- $z_{\mathcal{U}}\left(x^{3}\right)$

$-g(r, \lambda)$

- - level curves

Figure 2: Level curves of the functions $\max _{i \in[k]} \lambda_{i}\left(z_{i}-r_{i}\right)$ and $\min _{i \in[k]} \lambda_{i}\left(z_{i}-r_{i}\right)$ with $r=$ $(0,1)^{T}, \lambda=(3,4)^{T}$, which contain any $z(x, \xi)$ from Example 6 .

and that of the function $\min _{i \in[k]} \lambda_{i}\left(z_{i}-r_{i}\right)$ is

$$
\begin{aligned}
L_{\leqq}^{\min , r, \lambda}(\alpha) & =\left\{z \in \mathbb{R}^{k}: \min _{i \in[k]} \lambda_{i}\left(z_{i}-r_{i} \leqq \alpha\right\}\right. \\
& =\left\{z \in \mathbb{R}^{k}: \exists i \in[k] \text { with } z_{i} \leqq \frac{\alpha}{\lambda_{i}}+r_{i}\right\} \\
& =\left\{z \in \mathbb{R}^{k}: z \ngtr \alpha\left(\frac{1}{\lambda_{1}}, \ldots, \frac{1}{\lambda_{k}}\right)^{T}+r\right\} .
\end{aligned}
$$

Therefore, every sublevel set of $\max _{i \in[k]} \lambda_{i}\left(z_{i}-r_{i}\right)$ or $\min _{i \in[k]} \lambda_{i}\left(z_{i}-r_{i}\right)$ can be uniquely identified with a point on the line

$$
g(r, \lambda):=\left\{y(\alpha):=r+\alpha\left(\frac{1}{\lambda_{1}}, \ldots, \frac{1}{\lambda_{k}}\right)^{T}: \alpha \in \mathbb{R}\right\} .
$$

For two points $y(\alpha), y\left(\alpha^{\prime}\right) \in g(r, \lambda)$ we have $y(\alpha) \leq y\left(\alpha^{\prime}\right) \Leftrightarrow y(\alpha)<y\left(\alpha^{\prime}\right) \Leftrightarrow \alpha<\alpha^{\prime}$, because of $\lambda_{i}>0 \forall i \in[k]$. Figure 2 shows the level curves of $\max _{i \in[k]} \lambda_{i}\left(z_{i}-r_{i}\right)$ and $\min _{i \in[k]} \lambda_{i}\left(z_{i}-r_{i}\right)$ for $r=(0,0)^{T}$ and $\lambda=(2,1)^{T}$ that contain $z(x, \xi)$ for some $x \in \mathcal{X}$ and $\xi \in \mathcal{U}$ from Example 6 .

Recall the definitions of $\bar{z}(x)$ and $z_{\mathcal{U}}(x)$, used in the definition of pointMR efficiency and setMR efficiency (Definition 5). The following theorem shows that the optimal solutions for $(\mathrm{P}-\max (r, \lambda))$ can be identified by comparing the intersection points of $g(r, \lambda)$ with $\partial(\bar{z}(x)+$ $\left.\mathbb{R}_{\geqq}^{k}\right)$ for all $x \in \mathcal{X}$. Similarly, the optimal solutions of $(\mathrm{P}-\min (r, \lambda))$ can be identified by comparing the intersection points of $g(r, \lambda)$ with $\partial\left(z_{\mathcal{U}}(x)-\mathbb{R}_{\geqq}^{k}\right)$ for all $x \in \mathcal{X}$.

Theorem 10. Let $r \in \mathbb{R}^{k}, \lambda \in \mathbb{R}_{>}^{k}$ be given. A feasible solution $x^{*} \in \mathcal{X}$ is optimal for $(P-\max (r, \lambda))$ if and only if there exists $y^{*} \in \mathbb{R}^{k}$ such that $\left(x^{*}, y^{*}\right)$ is an efficient solution for

$$
\begin{aligned}
(G-\max (r, \lambda)) \quad \min & y \\
\text { s.t. } & y \in g(r, \lambda) \cap \partial\left(\bar{z}(x)+\mathbb{R}_{\geqq}^{k}\right) \\
x & \in \mathcal{X} .
\end{aligned}
$$



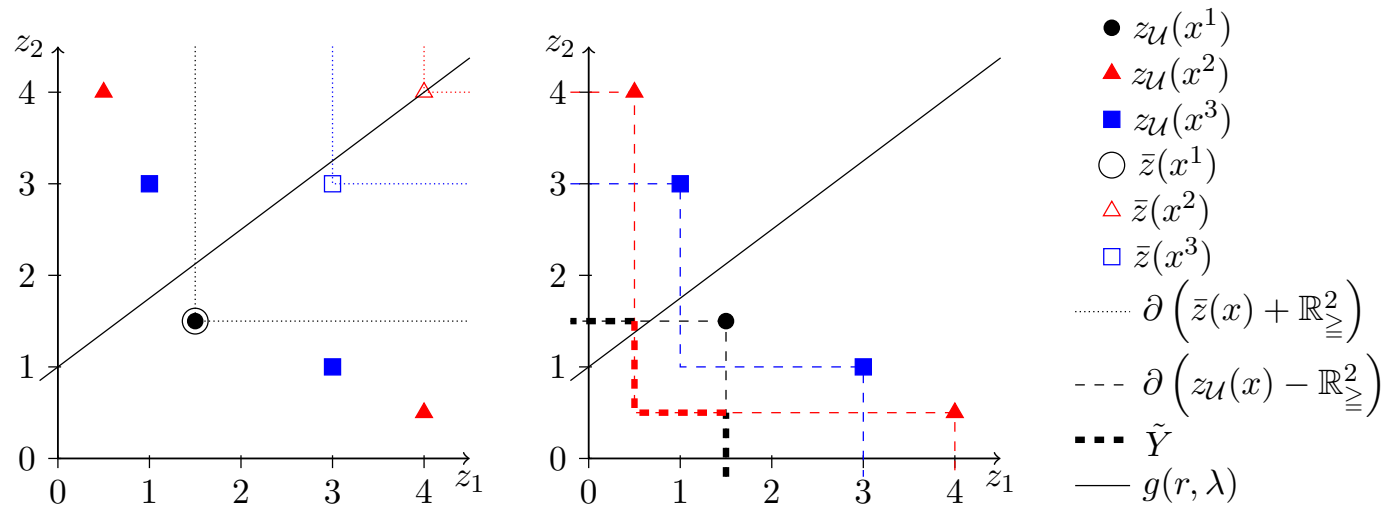

(a) Finding solutions for $(\mathrm{P}-\max (r, \lambda))$

(b) Finding solutions for $(\mathrm{P}-\min (r, \lambda))$

Figure 3: Determining the intersection point of $g(r, \lambda)$ with $\partial\left(\bar{z}(x)+\mathbb{R}_{\geqq}^{2}\right)$ (a) and $\partial\left(z_{\mathcal{U}}(x)-\mathbb{R}_{\geq}^{2}\right)$ (b) for the solutions in Example 6. As an example, $g(r, \lambda)$ is shown for $r=(0,1)^{T}, \lambda=(3,4)^{\bar{T}}$.

A feasible solution $x^{*} \in \mathcal{X}$ is optimal for $(P-\min (r, \lambda))$ if and only if there exists $y^{*} \in \mathbb{R}^{k}$ such that $\left(x^{*}, y^{*}\right)$ is an efficient solution for

$$
\begin{aligned}
(G-\min (r, \lambda)) \quad \min & y \\
\text { s.t. } y & \in g(r, \lambda) \cap \partial\left(z_{\mathcal{U}}(x)-\mathbb{R}_{\geqq}^{k}\right) \\
x & \in \mathcal{X} .
\end{aligned}
$$

Proof. We first show

$$
g(r, \lambda) \cap \partial\left(\bar{z}(x)+\mathbb{R}_{\geqq}^{k}\right)=\left\{r+\alpha^{\max }(x, r, \lambda)\left(\frac{1}{\lambda_{1}}, \ldots, \frac{1}{\lambda_{k}}\right)^{T}\right\}
$$

for every $x \in \mathcal{X}, r \in \mathbb{R}^{k}, \lambda \in \mathbb{R}_{>}^{k}$. For every $\alpha \in \mathbb{R}$ with $\alpha>\alpha^{\max }(x, r, \lambda)$ we have

$$
\begin{array}{cc}
\alpha>\alpha^{\max }(x, r, \lambda)=\max _{\xi \in \mathcal{U}} \max _{i \in[k]} \lambda_{i}\left(z_{i}(x, \xi)-r_{i}\right) \geqq \lambda_{i}\left(\max _{\xi \in \mathcal{U}} z_{i}(x, \xi)-r_{i}\right) & \forall i \in[k] \\
\Rightarrow r_{i}+\alpha \cdot \frac{1}{\lambda_{i}}>\max _{\xi \in \mathcal{U}} z_{i}(x, \xi)=\bar{z}_{i}(x) & \forall i \in[k] \\
\Rightarrow r+\alpha\left(\frac{1}{\lambda_{1}}, \ldots, \frac{1}{\lambda_{k}}\right)^{T} \in \bar{z}(x)+\mathbb{R}_{>}^{k}=\left(\bar{z}(x)+\mathbb{R}_{\geqq}^{k}\right) \backslash \partial\left(\bar{z}(x)+\mathbb{R}_{\geqq}^{k}\right) . &
\end{array}
$$

Further, for every $\alpha \in \mathbb{R}$ with $\alpha<\alpha^{\max }(x, r, \lambda)$,

$$
\begin{array}{rlr} 
& \alpha<\alpha^{\max }(x, r, \lambda)=\max _{\xi \in \mathcal{U}} \max _{i \in[k]} \lambda_{i}\left(z_{i}(x, \xi)-r_{i}\right) & \\
\Rightarrow & r_{i}+\alpha \cdot \frac{1}{\lambda_{i}}<\max _{\xi \in \mathcal{U}} z_{i}(x, \xi)=\bar{z}_{i}(x) & \\
\Rightarrow & r+\alpha\left(\frac{1}{\lambda_{1}}, \ldots, \frac{1}{\lambda_{k}}\right)^{T} \notin \bar{z}(x)+\mathbb{R}_{\geqq}^{k} & \text { for at least one } i \in[k] \\
\Rightarrow & r+\alpha\left(\frac{1}{\lambda_{1}}, \ldots, \frac{1}{\lambda_{k}}\right)^{T} \notin \partial\left(\bar{z}(x)+\mathbb{R}_{\geqq}^{k}\right) & \text { since } \bar{z}(x)+\mathbb{R}_{\geqq}^{k} \text { is closed. }
\end{array}
$$


It follows that $y\left(\alpha^{\max }(x, r, \lambda)\right)$ is the unique intersection point of $g(r, \lambda)$ with $\partial\left(\bar{z}(x)+\mathbb{R}_{\geq}^{k}\right)$. Hence, the only $y \in \mathbb{R}^{k}$, such that $(x, y)$ is feasible for $(\operatorname{G-max}(r, \lambda))$, is $y\left(\alpha^{\max }(x, r, \lambda)\right)$. It follows that

$$
\begin{aligned}
& x^{*} \text { is optimal for }(\mathrm{P}-\max (r, \lambda)) \\
\Leftrightarrow & \nexists x \in \mathcal{X}: \alpha^{\max }(x, r, \lambda)<\alpha^{\max }\left(x^{*}, r, \lambda\right) \\
\Leftrightarrow & \nexists x \in \mathcal{X}: y\left(\alpha^{\max }(x, r, \lambda)\right) \leq y\left(\alpha^{\max }\left(x^{*}, r, \lambda\right)\right) \\
\Leftrightarrow & \left(x^{*}, y\left(\alpha^{\max }\left(x^{*}, r, \lambda\right)\right)\right) \text { is an efficient solution for }(\mathrm{G}-\max (r, \lambda)) .
\end{aligned}
$$

Similarly, we show

$$
g(r, \lambda) \cap \partial\left(\bar{z}(x)+\mathbb{R}_{\geqq}^{k}\right)=\left\{r+\alpha^{\max }(x, r, \lambda)\left(\frac{1}{\lambda_{1}}, \ldots, \frac{1}{\lambda_{k}}\right)^{T}\right\}
$$

for every $x \in \mathcal{X}, r \in \mathbb{R}^{k}, \lambda \in \mathbb{R}_{>}^{k}$. For every $\alpha \in \mathbb{R}$ with $\alpha>\alpha^{\min }(x, r, \lambda)$ we have

$$
\begin{aligned}
& \alpha>\alpha^{\min }(x, r, \lambda) \geqq \min _{i \in[k]} \lambda_{i}\left(z_{i}(x, \xi)-r_{i}\right) \quad \forall \xi \in \mathcal{U} \\
& \Rightarrow \forall \xi \in \mathcal{U} \exists i \in[k]: r_{i}+\alpha \cdot \frac{1}{\lambda_{i}}>z_{i}(x, \xi) \\
& \Rightarrow r+\alpha\left(\frac{1}{\lambda_{1}}, \ldots, \frac{1}{\lambda_{k}}\right)^{T} \notin z(x, \xi)-\mathbb{R}_{\geqq}^{k} \\
& \Rightarrow r+\alpha\left(\frac{1}{\lambda_{1}}, \ldots, \frac{1}{\lambda_{k}}\right)^{T} \notin z \mathcal{U}(x)-\mathbb{R}_{\geqq}^{k} \\
& \Rightarrow r+\alpha\left(\frac{1}{\lambda_{1}}, \ldots, \frac{1}{\lambda_{k}}\right)^{T} \notin \partial\left(z_{\mathcal{U}}(x)-\mathbb{R}_{\geqq}^{k}\right), \quad \text { since } z_{\mathcal{U}}(x)-\mathbb{R}_{\geqq}^{k} \text { is closed, }
\end{aligned}
$$

and for every $\alpha \in \mathbb{R}$ with $\alpha<\alpha^{\min }(x, r, \lambda)$,

$$
\alpha<\alpha^{\min }(x, r, \lambda)=\min _{i \in[k]} \lambda_{i}\left(z_{i}(x, \xi)-r_{i}\right)
$$

$\Rightarrow \exists \xi \in \mathcal{U}$ such that $\forall i \in[k]: r_{i}+\alpha \cdot \frac{1}{\lambda_{i}}<z_{i}(x, \xi)$

$\Rightarrow \exists \xi \in \mathcal{U}: r+\alpha\left(\frac{1}{\lambda_{1}}, \ldots, \frac{1}{\lambda_{k}}\right)^{T} \in z(x, \xi)-\mathbb{R}_{>}^{k}$

$\Rightarrow r+\alpha\left(\frac{1}{\lambda_{1}}, \ldots, \frac{1}{\lambda_{k}}\right)^{T} \in z_{\mathcal{U}}(x)-\mathbb{R}_{>}^{k}=\left(z_{\mathcal{U}}(x)-\mathbb{R}_{\geqq}^{k}\right) \backslash \partial\left(z_{\mathcal{U}}(x)-\mathbb{R}_{\geqq}^{k}\right)$.

Hence, for all $x \in \mathcal{X}, y\left(\alpha^{\max }(x, r, \lambda)\right)$ is the unique intersection point of $g(r, \lambda)$ with $\partial(z \mathcal{U}(x)-$ $\left.\mathbb{R}_{\geq}^{k}\right)$. Therefore, $x^{*}$ is optimal for $(\mathrm{P}-\max (r, \lambda))$ if and only if $\left(x^{*}, y\left(\alpha^{\max }\left(x^{*}, r, \lambda\right)\right)\right)$ is an efficient solution for $(\mathrm{G}-\max (r, \lambda))$.

Note that it follows from the proof of Theorem 10 that for $(\mathrm{G}-\max (r, \lambda))$ and $(\mathrm{G}-\min (r, \lambda))$ every weakly efficient solution is also efficient, because we have $y(\alpha) \leq y\left(\alpha^{\prime}\right) \Leftrightarrow y(\alpha)<y\left(\alpha^{\prime}\right)$ for two points $y(\alpha), y\left(\alpha^{\prime}\right) \in g(r, \lambda)$. Theorem 10 implies, that a solution $x \in \mathcal{X}$ can be found with the [max-ordering/min-ordering] method if and only if there exist $\lambda \in \mathbb{R}_{>}^{k}, r \in \mathbb{R}^{k}, y \in \mathbb{R}^{k}$, such that $(x, y)$ is (weakly) efficient for $[(\mathrm{G}-\max (r, \lambda)) /(\mathrm{G}-\min (r, \lambda))]$. 
Figure 3 illustrates $g(r, \lambda), \partial\left(\bar{z}(x)+\mathbb{R}_{\geqq}^{k}\right)$ and $\partial\left(z_{\mathcal{U}}(x)-\mathbb{R}_{\geqq}^{k}\right)$ for the feasible solutions in Example 6. It is easy to see in Figure 3(a) that for each choice of $r, \lambda$ the intersection point of $g(r, \lambda)$ with $\partial\left(\bar{z}\left(x^{1}\right)+\mathbb{R}_{\geq}^{k}\right)$ has smaller coordinates than the intersection point of $g(r, \lambda)$ with $\partial\left(\bar{z}\left(x^{2}\right)+\mathbb{R}_{\geqq}^{k}\right)$ or $\partial\left(\bar{z}\left(x^{3}\right)+\mathbb{R}_{\geqq}^{k}\right)$, hence $x^{1}$ is the unique optimal solution for $(\mathrm{P}-\max (r, \lambda))$. Let us now consider the sets

$$
Y:=\bigcup_{x \in \mathcal{X}} \partial\left(z_{\mathcal{U}}(x)-\mathbb{R}_{\geqq}^{k}\right) \text { and } \tilde{Y}:=\left\{y \in Y: \nexists y^{\prime} \in Y: y^{\prime}<y\right\} .
$$

For each $y \in \tilde{Y}$ there exists $r \in \mathbb{R}^{k}, \lambda \in \mathbb{R}_{>}^{k}, x \in \mathcal{X}$ such that $(x, y)$ is efficient for $(\mathrm{G}-\min (r, \lambda))$ : choose $r=y$, then $y \in g(r, \lambda)$, hence there exists $x$ such that $(x, y)$ is feasible for $(\mathrm{G}-\min (r, \lambda))$, because $y \in Y$. Further there is no feasible $\left(x^{\prime}, y^{\prime}\right)$ with $y^{\prime}<y$, because $y \in \tilde{Y}$, hence $(x, y)$ is (weakly) efficient for $(\mathrm{G}-\min (r, \lambda))$.

Figure 3(b) shows $\partial\left(z_{\mathcal{U}}(x)-\mathbb{R}_{\geqq}^{k}\right)$ for all $x \in \mathcal{X}$ in Example 6 as dashed lines and $\tilde{Y}$ as thick dashed line. Since $\partial\left(z_{\mathcal{U}}\left(x^{1}\right)-\mathbb{R}_{\geqq}^{k}\right) \cap \tilde{Y}$ and $\partial\left(z_{\mathcal{U}}\left(x^{2}\right)-\mathbb{R}_{\geqq}^{k}\right) \cap \tilde{Y}$ are not empty, $x^{1}$ and $x^{2}$ can be found with the min-ordering method. On the other hand, it is easy to see that for every $r \in \mathbb{R}^{k}, \lambda \in \mathbb{R}_{>}^{k}$ there exists a point $\tilde{y} \in \tilde{Y} \cap g(r, \lambda)$ and that $\tilde{y} \leq y$ for $y \in g(r, \lambda) \cap \partial\left(z_{\mathcal{U}}\left(x^{3}\right)-\mathbb{R}_{\geq}^{k}\right)$. Therefore, $x^{3}$ is not optimal for $(\mathrm{P}-\min (r, \lambda))$.

\subsection{Solutions found with the max-ordering method}

[Ide14] show that (for fixed reference point 0 ) every $[\cdot /$ unique] solution of $(\mathrm{P}-\max (r, \lambda))$ is setMR [weakly/strictly] efficient. We show that for every reference point $r \in \mathbb{R}^{k}$ every [·/unique] solution of the max-ordering optimization problem is even pointMR [weakly/strictly] efficient and that for a small enough $r$ all pointMR weakly efficient solutions can be found by choosing an appropriate $\lambda$.

Theorem 11. Let $r \in \mathbb{R}^{k}, \lambda \in \mathbb{R}_{>}^{k}$ be given and let $x$ be an optimal solution for $(P-\max (r, \lambda))$. Then

1. $x$ is pointMR weakly efficient for $(P)$ and

2. if $x$ is the unique optimal solution for $(P-\max (r, \lambda))$, then $x$ is pointMR strictly efficient.

Proof. Let $x$ be [an/the unique] optimal solution for $(\mathrm{P}-\max (r, \lambda))$. Assume that $x$ is not pointMR [weakly/strictly] efficient. Then there exists a solution $x^{\prime} \in \mathcal{X}$ with

$$
\begin{array}{ccc}
\max _{\xi \in \mathcal{U}} z_{i}\left(x^{\prime}, \xi\right)[</ \leqq] & \forall i \in[k] \\
\Leftrightarrow & \max _{\xi \in \mathcal{U}} z_{i}(x, \xi) & \forall i \in[k] \\
\Rightarrow & \max _{\xi \in \mathcal{U}} \lambda_{i}\left(z_{i}\left(x^{\prime}, \xi\right)-r_{i}\right)[</ \leqq] \max _{\xi \in \mathcal{U}} \lambda_{i}\left(z_{i}(x, \xi)-r_{i}\right) & \\
\Rightarrow & \max _{\xi \in[k]} \max _{i}\left(z_{i}\left(x^{\prime}, \xi\right)-r_{i}\right)[</ \leqq] \max _{i \in[k]} \max _{\xi \in \mathcal{U}} \lambda_{i}\left(z_{i}(x, \xi)-r_{i}\right) & \\
\Rightarrow & \left.\max _{i \in[k]}\left(x^{\prime}, \xi\right)-r_{i}\right)[</ \leqq] \max _{\xi \in \mathcal{U}} \max _{i \in[k]} \lambda_{i}\left(z_{i}(x, \xi)-r_{i}\right) &
\end{array}
$$

1. If $x$ is not pointMR weakly efficient, i.e., $<$ holds, this is a contradiction to $x$ being an optimal solution for $(\mathrm{P}-\max (r, \lambda))$.

2. If $x$ is not pointMR strictly efficient, i.e., $\leqq$ holds, then $x$ is not optimal for $(\mathrm{P}-\max (r, \lambda))$ or $x^{\prime}$ is optimal as well. This contradicts $x$ being the unique optimal solution. 
Theorem 11 implies that not all setMR weakly efficient solutions can be found with the maxordering method, because a setMR weakly efficient solution is not necessarily pointMR weakly efficient. However, the following theorem shows that for a suitable choice of $r$ all pointMR weakly efficient solutions can be found by varying $\lambda$.

Theorem 12. Let $x$ be a pointMR weakly efficient solution and let a reference point $r \in \mathbb{R}^{k}$ with $r_{i}<\max _{\xi \in \mathcal{U}} z_{i}(x, \xi) \forall i \in[k]$ be given. Then there exists a weight vector $\lambda \in \mathbb{R}_{>}^{k}$ such that $x$ is an optimal solution for $(P-\max (r, \lambda))$.

Proof. Because of $r_{i}<\max _{\xi \in \mathcal{U}} z_{i}(x, \xi)$ we obtain well-defined and positive weights by setting

$$
\lambda_{i}:=\frac{1}{\max _{\xi \in \mathcal{U}} z_{i}(x, \xi)-r_{i}} \forall i=1, \ldots, k
$$

It follows that $\max _{\xi \in \mathcal{U}} \lambda_{i}\left(z_{i}(x, \xi)-r_{i}\right)=\lambda_{i}\left(\max _{\xi \in \mathcal{U}} z_{i}(x, \xi)-r_{i}\right)=1 \forall i \in[k]$.

Let $x^{\prime} \in X$ be any feasible solution. Since $x$ is weakly pointMR efficient, there exists at least one index $j \in\{1, \ldots, k\}$ with $\max _{\xi \in \mathcal{U}} z_{j}(x, \xi) \leqq \max _{\xi \in \mathcal{U}} z_{j}\left(x^{\prime}, \xi\right)$. It follows that

$$
\begin{aligned}
\max _{i \in[k]} \max _{\xi \in \mathcal{U}} \lambda_{i}\left(z_{i}(x, \xi)-r_{i}\right)=1 & =\max _{\xi \in \mathcal{U}} \lambda_{j}\left(z_{j}(x, \xi)-r_{j}\right)=\lambda_{j}\left(\max _{\xi \in \mathcal{U}} z_{j}(x, \xi)-r_{j}\right) \\
& \leqq \lambda_{j}\left(\max _{\xi \in \mathcal{U}} z_{j}\left(x^{\prime}, \xi\right)-r_{j}\right)=\max _{\xi \in \mathcal{U}} \lambda_{j}\left(z_{j}\left(x^{\prime}, \xi\right)-r_{j}\right) \\
& \leqq \max _{i \in[k]} \max _{\xi \in \mathcal{U}} \lambda_{i}\left(z_{i}\left(x^{\prime}, \xi\right)-r_{i}\right),
\end{aligned}
$$

hence, $x$ is optimal for $(\operatorname{Pmax}(r, \lambda))$.

The results from Section 3.1 provide a geometric interpretation of the proof of Theorem 12: For given $r, x$ and the $\lambda$ constructed in the proof of Theorem $12, g(r, \lambda)$ is the line through $r$ and $\bar{z}(x)$. Then, $\bar{z}(x)=y\left(\alpha^{\max }(x, r, \lambda)\right)$ and $y(\alpha) \in \bar{z}(x)-\mathbb{R}_{>}^{k}$ for all $\alpha<\left(\alpha^{\max }(x, r, \lambda)\right)$. If $x$ is pointMR efficient, $\bar{z}(x)-\mathbb{R}_{\geq}^{k} \cap \partial\left(\bar{z}\left(x^{\prime}\right)+\mathbb{R}_{\geq}^{k}\right)$ is empty for all $\bar{x}^{\prime} \in \mathcal{X}$, hence $\left(x, y\left(\alpha^{\max }(x, r, \lambda)\right)\right.$ is an efficient solution for $(\mathrm{G}-\max (r, \lambda))$.

\subsection{Solutions found with the min-ordering method}

For $(\mathrm{P}-\min (r, \lambda))$ we show that every [//unique] solution is set-based robust [weakly/strictly] efficient, i.e., the min-ordering scalarization method is suitable for finding setMR (weakly) efficient efficient solutions. Moreover, we show that with this method we can find setMR efficient solutions that cannot be found with the other known scalarization methods presented in Section 2.2, including the weighted sum, $\epsilon$-constraint and augmented weighted Chebychev method. This also implies that solutions for $(\mathrm{P}-\min (r, \lambda))$ are not necessarily pointMR efficient. In addition, we briefly discuss the connection to adjustable robustness.

Theorem 13. Let $r \in \mathbb{R}^{k}, \lambda \in \mathbb{R}_{>}^{k}$ be given and let $x$ be an optimal solution for $(P-\min (r, \lambda))$. Then

1. $x$ is setMR weakly efficient for $(P)$ and

2. if $x$ is the unique optimal solution for $(P-\min (r, \lambda))$, then $x$ is setMR strictly efficient. 
Proof. Let $x$ be [an/the unique] optimal solution for $(\mathrm{P}-\min (r, \lambda))$. Assume that $x$ is not setMR [weakly/strictly] efficient. From Lemma 7 it follows that there exists a feasible solution $x^{\prime}$ with $\forall \xi \in \mathcal{U} \exists \eta \in \mathcal{U}: z\left(x^{\prime}, \xi\right)[</ \leqq] z(x, \eta)$. Let $\xi^{\prime} \in \operatorname{argmax}_{\xi \in \mathcal{U}} \min _{i \in[k]} \lambda_{i}\left(z_{i}\left(x^{\prime}, \xi\right)-r_{i}\right)$ be a worst case scenario of $x^{\prime}$ w.r.t. $(\mathrm{P}-\min (r, \lambda))$ Then there exists $\eta^{\prime} \in \mathcal{U}$ with

$$
\begin{aligned}
& z_{i}\left(x^{\prime}, \xi^{\prime}\right)[</ \leqq] z_{i}\left(x, \eta^{\prime}\right) \quad \forall i \in[k] \\
& \Leftrightarrow \quad \lambda_{i}\left(z_{i}\left(x^{\prime}, \xi^{\prime}\right)-r_{i}\right)[</ \leqq] \lambda_{i}\left(z_{i}\left(x, \eta^{\prime}\right)-r_{i}\right) \quad \forall i \in[k]
\end{aligned}
$$

We hence conclude that

$$
\begin{aligned}
& \max _{\xi \in \mathcal{U}} \min _{i \in[k]} \lambda_{i}\left(z_{i}\left(x^{\prime}, \xi\right)-r_{i}\right)=\min _{i \in[k]} \lambda_{i}\left(z_{i}\left(x^{\prime}, \xi^{\prime}\right)-r_{i}\right) \\
& {[</ \leqq] } \min _{i \in[k]} \lambda_{i}\left(z_{i}\left(x, \eta^{\prime}\right)-r_{i}\right) \\
& \leqq \\
& \max _{\xi \in \mathcal{U}} \min _{i \in[k]} \lambda_{i}\left(z_{i}(x, \xi)-r_{i}\right) .
\end{aligned}
$$

1. If $x$ is not setMR weakly efficient, i.e., $<$ holds, this is a contradiction to $x$ being an optimal solution for $(\mathrm{P}-\min (r, \lambda))$.

2. If $x$ is not setMR strictly efficient, i.e., $\leqq$ holds, then $x$ is not optimal for $(\mathrm{P}-\min (r, \lambda))$ or $x^{\prime}$ is optimal as well. This contradicts $x$ being the unique optimal solution of $(\mathrm{P}-\min (r, \lambda))$.

Theorem 14. There exists a multi-objective uncertain optimization problem with setMR efficient solutions that cannot be found

- with the $\epsilon$-constraint method,

- with the p-norm scalarization method,

- or by solving any scalarized problem of the form

$$
\min _{x \in \mathcal{X}}\left(\rho_{1} \max _{\xi \in \mathcal{U}} \max _{i \in[k]} \nu_{i}\left(z_{i}(x, \xi)-r_{i}\right)+\rho_{2} \max _{\xi \in \mathcal{U}} \sum_{i \in[k]} \mu_{i}\left(z_{i}(x, \xi)-r_{i}\right)\right)
$$

with $\rho \in \mathbb{R}_{\geq}^{2}, \nu, \mu \in \mathbb{R}_{>}^{k}, r_{i} \in \mathbb{R}^{k}$.

Some, but not all, of these solutions can be found with the min-ordering optimization method.

Proof. Consider the multi-objective uncertain optimization problem given in Example 6. Recall that all three solutions are setMR efficient. Because of

$$
\begin{aligned}
& \left(\max _{\xi \in \mathcal{U}} z_{1}\left(x^{1}, \xi\right), \max _{\xi \in \mathcal{U}} z_{2}\left(x^{1}, \xi\right)\right)=(1.5,1.5) \\
<\left(\max _{\xi \in \mathcal{U}} z_{1}\left(x^{3}, \xi\right), \max _{\xi \in \mathcal{U}} z_{2}\left(x^{3}, \xi\right)\right) & =(3,3) \\
<\left(\max _{\xi \in \mathcal{U}} z_{1}\left(x^{2}, \xi\right), \max _{\xi \in \mathcal{U}} z_{2}\left(x^{2}, \xi\right)\right) & =(4,4),
\end{aligned}
$$


$x^{1}$ is the only pointMR weakly efficient solution, hence only $x^{1}$ can be found with the $\epsilon$ constraint method ([EIS14]).

[BF17] show that a solution $x \in \mathcal{X}$ can only be found with the p-norm scalarization method if

$$
\nexists x^{\prime} \in \mathcal{X}: z_{\mathcal{U}}\left(x^{\prime}\right) \in \operatorname{Conv}\left(z_{\mathcal{U}}(x)\right)-\mathbb{R}_{>}^{k},
$$

where $\operatorname{Conv}\left(z_{\mathcal{U}}(x)\right)$ denotes the convex hull of $z_{\mathcal{U}}(x)$. Since $(1.5,1.5) \in \operatorname{Conv}(\{(1,3),(3,1)\})-$ $\mathbb{R}_{>}^{k}$ and $(1.5,1.5) \in \operatorname{Conv}(\{(0.5,4),(4,0.5)\})-\mathbb{R}_{>}^{k}, x^{1}$ is the only solution that can be found with the p-norm scalarization method.

Let now $\rho \in \mathbb{R}_{\geq}^{2}, \nu, \mu \in \mathbb{R}_{>}^{k}, r_{i} \in \mathbb{R}^{k}$ be given and consider the scalarized problem (1). We define

$$
\begin{aligned}
f(x) & :=\max _{\xi \in \mathcal{U}} \max _{i \in[k]} \nu_{i}\left(z_{i}(x, \xi)-r_{i}\right) \text { and } \\
h(x) & :=\max _{\xi \in \mathcal{U}} \sum_{i \in[k]} \mu_{i}\left(z_{i}(x, \xi)-r_{i}\right)
\end{aligned}
$$

From Theorem 11 it follows that only $x^{1}$ can be optimal for $\min _{x \in \mathcal{X}} f(x)$, because it is the only pointMR weakly efficient solution. In the following we show that $x^{1}$ is also the only optimal solution for $\min _{x \in \mathcal{X}} h(x)$. Let $\mu \in \mathbb{R}_{>}^{2}, \mu_{i} \geqq \mu_{j},\{i, j\}=\{1,2\}$. Then

$$
\begin{array}{lll}
h\left(x^{1}\right)=1.5 \mu_{1}+1.5 \mu_{2}-\mu_{1} r_{1}-\mu_{2} r_{2} & \leqq 3 \mu_{i}-\mu_{1} r_{1}-\mu_{2} r_{2} \\
h\left(x^{3}\right)=\max \left\{3 \mu_{1}+\mu_{2}, \mu_{1}+3 \mu_{2}\right\}-\mu_{1} r_{1}-\mu_{2} r_{2} & >3 \mu_{i}-\mu_{1} r_{1}-\mu_{2} r_{2} \\
h\left(x^{2}\right)=\max \left\{4 \mu_{1}+0.5 \mu_{2}, 0.5 \mu_{1}+4 \mu_{2}\right\}-\mu_{1} r_{1}-\mu_{2} r_{2} & >3 \mu_{i}-\mu_{1} r_{1}-\mu_{2} r_{2}
\end{array}
$$

It follows that $x^{1}$ is the unique optimal solution for $\min _{x \in \mathcal{X}} h(x)$. Since it is also uniquely optimal for $\min _{x \in \mathcal{X}} f(x), x^{1}$ is the unique optimal solution for (1) for every $\rho \in \mathbb{R}_{>}^{2}$.

We conclude that the setMR efficient solutions $x^{2}$ and $x^{3}$ cannot be found with any of the methods listed in the statement.

In Figure 3(b) it is easy to see that there exists no $r \in \mathbb{R}^{k}$ and $\lambda \in \mathbb{R}_{>}^{k}$, such that the minimal intersection point of $g(r, \lambda)$ with $\bigcup_{x \in \mathcal{X}} \partial\left(z_{\mathcal{U}}(x)-\mathbb{R}_{\geq}^{k}\right)$ is in $\partial\left(z_{\mathcal{U}}\left(x^{3}\right)-\mathbb{R}_{\geq}^{k}\right)$. With Theorem 10 it follows that $x^{3}$ cannot be found with the min-ordering scalarization method either.

However, $x^{2}$ is optimal for $(\mathrm{P}-\min (r, \lambda))$ with $r=(0,0)^{T}, \lambda=(1,1)^{T}$, because

$$
\max _{\xi \in \mathcal{U}} \min _{i=1,2} z_{i}\left(x^{2}, \xi\right)=0.5<\max _{\xi \in \mathcal{U}} \min _{i=1,2} z_{i}\left(x^{3}, \xi\right)=1<\max _{\xi \in \mathcal{U}} \min _{i=1,2} z_{i}\left(x^{1}, \xi\right)=1.5 .
$$

Remark 15. Note that $(P-\min (r, \lambda))$ can be interpreted as a special case of a (single-objective) two-stage or adjustable robust problem, where $x \in \mathcal{X}$ must be chosen here-and-now, i.e., before the realization of the uncertain parameters, whereas the relevant objective may be chosen afterwards (wait-and-see). This can be modeled by introducing an additional variable $y$, which determines the choice of the objective. However, the additional variable changes the structure of the underlying problem $P$, e.g., if $\mathcal{X}$ is the feasible set of a particular combinatorial problem (e.g., the shortest path problem), the feasible set $\mathcal{X} \times\{1, \ldots, k\}$ does not have the same structure (e.g., is not necessarily equivalent to the set of all paths in a graph). Also, the additional variable is integer, such that solution methods for robust adjustable counterparts of linear programs cannot be used to solve $(P-\min (r, \lambda))$. 


\section{Min-ordering and max-ordering optimization problem for multi-objective combinatorial problems}

In Section 3 we have shown that all pointMR efficient solutions can be found with the maxordering method, and the min-ordering method finds setMR efficient solutions, some of which are not found with any of the formerly developed scalarization based methods (see Section 2.2). On an example, we have shown the meaning of the particular solutions obtained with the min-ordering and the max-ordering method.

Now, we investigate how the problems $(\mathrm{P}-\min (r, \lambda))$ and $(\mathrm{P}-\max (r, \lambda))$ can be solved for combinatorial problems. We show that in case of interval uncertainty the uncertainty set can be reduced to one scenario, resulting in problems which have already been considered in the literature. For a multi-objective extension of the so-called bounded uncertainty set we develop compact mixed-integer linear programming (MILP) formulations, i.e., formulations without nested minimum and maximum functions.

We consider multi-objective combinatorial problems with uncertain costs (MOUCO): Let a finite set of elements $E=\left\{e_{1}, \ldots, e_{n}\right\}$ and a feasible set $\mathcal{X} \subseteq\{0,1\}^{n}$ be given. Each feasible solution $x \in \mathcal{X}$ represents a subset of $E$, which contains element $e_{j}$ if and only if $x_{j}=1$. Further, a cost matrix $c \in \mathbb{R}^{k \times n}$ is given, assigning a cost $c_{i, j}$ to element $e_{j}$ in the $i$-th objective function for $i \in[k]$. The costs are uncertain, i.e., $c \in \mathcal{U} \subseteq \mathbb{R}^{k \times n}$. The $k$ objective functions $z_{i}(x, c)$ hence depend on $x$ and on the realization of the costs and are given as

$$
z_{i}(x, c):=\sum_{j \in[n]} c_{i, j} x_{j} \forall x \in \mathcal{X}, c \in \mathcal{U} .
$$

For a solution $x \in \mathcal{X}$ we write $|x|:=\sum_{j \in[n]} x_{j}$.

\subsection{Interval uncertainty}

We use a straight-forward extension of the often used single-objective concept of interval uncertainty, where each uncertain parameter takes any value in a given interval, independent of the realization of the other parameters.

Definition 16. Let lower bounds $\hat{c} \in \mathbb{R}^{k \times n}$ and interval lengths $\delta \in \mathbb{R}_{\geqq}^{k \times n}$ be given. We define the interval uncertainty set

$$
\mathcal{U}^{I}:=\left\{c \in \mathbb{R}^{k \times n}: c_{i, j}=\hat{c}_{i, j}+\beta_{i, j} \delta_{i, j}, \beta_{i, j} \in[0,1] \forall i \in[k], j \in[n]\right\} .
$$

The following theorem shows that in case of interval uncertainty it is sufficient to consider the upper bounds of the intervals, i.e., the uncertainty set can be reduced to a single scenario. Therefore, $(\mathrm{P}-\min (r, \lambda))$ can be solved by solving $k$ single-objective deterministic combinatorial problems and $(\mathrm{P}-\max (r, \lambda))$ can be interpreted as a single-objective minmax robust combinatorial problem with discrete uncertainty set (see Remark 9).

Theorem 17. Let $(P)$ be a MOUCO with uncertainty set $\mathcal{U}:=\mathcal{U}^{I}$. We define $\bar{c}_{i, j}:=\hat{c}_{i, j}+\delta_{i, j}$ for all $j \in[n], i \in[k]$. Then $(P-\min (r, \lambda))$ is equivalent to

$$
\min _{x \in \mathcal{X}, i \in[k]} \lambda_{i}\left(z_{i}(x, \bar{c})-r_{i}\right)
$$


and $(P-\max (r, \lambda))$ is equivalent to

$$
\min _{x \in \mathcal{X}} \max _{i \in[k]} \lambda_{i}\left(z_{i}(x, \bar{c})-r_{i}\right) .
$$

Proof. From $\bar{c} \in \mathcal{U}^{I}$ and $c_{i, j} \leqq \bar{c}_{i, j} \forall c \in \mathcal{U}^{I}, j \in[n], i \in[k]$ we conclude

$$
\begin{array}{rlrlrl}
\lambda_{i}\left(z_{i}(x, c)-r_{i}\right) & \leqq \lambda_{i}\left(z_{i}(x, \bar{c})-r_{i}\right) & & \forall x \in \mathcal{X}, c \in \mathcal{U}^{I}, i \in[k] \\
\Rightarrow & \min _{i \in[k]} \lambda_{i}\left(z_{i}(x, c)-r_{i}\right) & \leqq \min _{i \in[k]} \lambda_{i}\left(z_{i}(x, \bar{c})-r_{i}\right) & & \forall x \in \mathcal{X}, c \in \mathcal{U}^{I} \\
\stackrel{\bar{c} \in \mathcal{U}^{I}}{\Rightarrow} & \max _{c \in \mathcal{U}^{I}} \min _{i \in[k]} \lambda_{i}\left(z_{i}(x, c)-r_{i}\right) & =\min _{i \in[k]} \lambda_{i}\left(z_{i}(x, \bar{c})-r_{i}\right) & & \forall x \in \mathcal{X} \\
\Rightarrow & \min _{x \in \mathcal{X}} \max _{c \in \mathcal{U}^{I}} \min _{i \in[k]} \lambda_{i}\left(z_{i}(x, c)-r_{i}\right) & =\min _{x \in \mathcal{X}} \min _{i \in[k]} \lambda_{i}\left(z_{i}(x, \bar{c})-r_{i}\right), & &
\end{array}
$$

where all minima and maxima exist due to the finiteness of $\mathcal{X}$, the compactness of $\mathcal{U}^{I}$ and the continuity of $z(x, \cdot): \mathcal{U}^{I} \rightarrow \mathbb{R}$. For $(\mathrm{P}-\max (r, \lambda))$ we analogously obtain

$$
\min _{x \in \mathcal{X}^{\mathcal{X}}} \max _{c \in \mathcal{U}^{I}} \max _{i \in[k]} \lambda_{i}\left(z_{i}(x, c)-r_{i}\right)=\min _{x \in \mathcal{X}} \max _{i \in[k]} \lambda_{i}\left(z_{i}(x, \bar{c})-r_{i}\right) .
$$

It follows that $(\mathrm{P}-\min (r, \lambda))$ with interval uncertainty set $\mathcal{U}^{I}$ is polynomially solvable if the single-objective deterministic problem can be solved in polynomial time. However, $(\mathrm{P}-\max (r, \lambda))$ is as complex as a single-objective minmax robust problem with discrete uncertainty set. This has been shown to be NP-hard for several combinatorial problems, which can be solved in polynomial time in the single-objective deterministic case, e.g., the shortest path, minimum spanning tree and assignment problem, see [KY97].

\subsection{Bounded uncertainty}

The concept of bounded uncertainty, also called $\Gamma$-uncertainty or cardinality constrained uncertainty, was introduced for single-objective optimization by [BS03]. Its idea is that it is unlikely that all uncertain parameters, which vary in intervals, attain their worst case value simultaneously. Therefore, the authors assume that not more than $\Gamma$ parameters differ from their so-called nominal value.

We extend this idea to multi-objective uncertain combinatorial optimization by assuming, that at most a given number $\Gamma$ of all cost parameters will deviate from their minimal value.

Definition 18. Let $\hat{c} \in \mathbb{R}^{k \times n}, \delta \in \mathbb{R}_{\geqq}^{k \times n}$ and $\Gamma \in \mathbb{Z}$ with $0 \leqq \Gamma \leqq(n \cdot k)$ be given. We define the discretely bounded uncertainty set as

$$
\mathcal{U}^{d}:=\left\{c \in \mathbb{R}^{k \times n}: c_{i, j}=\hat{c}_{i, j}+\beta_{i, j} \delta_{i, j}, \beta_{i, j} \in\{0,1\} \forall i \in[k], j \in[n], \sum_{i \in[k], j \in[n]} \beta_{i, j} \leqq \Gamma\right\}
$$

[BS03] also allow more than $\Gamma$ parameters to deviate from their minimal value if not all attain their maximal value, but deviate to a lesser extend. In the single-objective robust optimization case treated in [BS03], restricting to what extent the parameters may deviate in total leads to the same objective value as restricting the number of deviating parameters. However, Example 22 shows that this does not hold for $(\mathrm{P}-\min (r, \lambda))$. Therefore, we also consider the continuously bounded uncertainty set: 
Definition 19. Let $\hat{c} \in \mathbb{R}^{k \times n}, \delta \in \mathbb{R}_{\geqq}^{k \times n}$ and $\Gamma \in \mathbb{Z}$ with $0 \leqq \Gamma \leqq(n \cdot k)$ be given. We define the continuously bounded uncertainty set

$$
\mathcal{U}^{c}:=\left\{c \in \mathbb{R}^{k \times n}: c_{i, j}=\hat{c}_{i, j}+\beta_{i, j} \delta_{i, j}, \beta_{i, j} \in[0,1] \forall i \in[k], j \in[n], \sum_{i \in[k], j \in[n]} \beta_{i, j} \leqq \Gamma\right\}
$$

If we can assume that the uncertainties in the objectives are independent of each other, another possibility to extend the idea of bounded uncertainty to multi-objective optimization is to restrict the deviation of the parameters for each objective independently. This has been done in [HNS13, RSST18]. They use an objective-wise extension of the concept of bounded uncertainty, which we will refer to as objective-wise bounded uncertainty.

Definition 20. Let $\hat{c} \in \mathbb{R}^{k \times n}, \delta \in \mathbb{R}_{\geqq}^{k \times n}$ and $\Gamma_{i} \in \mathbb{Z}$ with $0 \leqq \Gamma_{i} \leqq n \forall i \in[k]$ be given. We define the objective-wise bounded uncertainty set

$\mathcal{U}^{\text {owb }}:=\left\{c \in \mathbb{R}^{k \times n}: c_{i, j}=\hat{c}_{i, j}+\beta_{i, j} \delta_{i, j}, \beta_{i, j} \in[0,1] \forall i \in[k], j \in[n], \sum_{j \in[n]} \beta_{i, j} \leqq \Gamma_{i} \forall i \in[k]\right\}$.

In the following, we focus on discretely and continuously bounded uncertainty.

\subsubsection{MILP-formulation for $(\mathrm{P}-\max (r, \lambda))$ with bounded uncertainty}

In this section we introduce a MILP-formulation for $(\mathrm{P}-\max (r, \lambda))$ with discretely or continuously bounded uncertainty set. We show that we can apply the same approach that [HNS13] use to develop an augmented weighted Chebyshev method for multi-objective uncertain linear problems with objective-wise bounded uncertainty set.

In the following we show that for $(\mathrm{P}-\max (r, \lambda))$ we do not need to distinguish between the uncertainty sets $\mathcal{U}^{d}$ and $\mathcal{U}^{c}$. Moreover, even using $\mathcal{U}^{\text {owb }}$ results in an equivalent problem, if the bound $\Gamma_{i}$ is the same for all objectives.

Lemma 21. For given $\mathcal{X} \subseteq\{0,1\}^{n}, \lambda \in \mathbb{R}_{>}^{k}, r \in \mathbb{R}^{k}, \hat{c}, \delta \in \mathbb{R}^{k \times n}, \Gamma=\Gamma_{1}=\ldots=\Gamma_{k} \in \mathbb{Z}_{\geqq}$:

$$
\begin{aligned}
\min _{x \in \mathcal{X}^{\mathcal{X}}} \max _{c \in \mathcal{U}^{d}} \max _{i \in[k]} \lambda_{i}\left(z_{i}(x, c)-r_{i}\right) & =\min _{x \in \mathcal{X}} \max _{c \in \mathcal{U}^{c}} \max _{i \in[k]} \lambda_{i}\left(z_{i}(x, c)-r_{i}\right) \\
& =\min _{x \in \mathcal{X}} \max _{\xi \in \mathcal{U}^{\text {owb }}} \max _{i \in[k]} \lambda_{i}\left(z_{i}(x, c)-r_{i}\right) .
\end{aligned}
$$

Proof. Let $x \in \mathcal{X}, i \in[k]$ be given. Let $\pi:[n] \rightarrow[n]$ be a permutation such that $\delta_{i, \pi(1)} x_{\pi(1)} \geqq$ $\delta_{i, \pi(2)} x_{\pi(2)} \geqq \ldots \geqq \delta_{i, \pi(n)} x_{\pi(n)}$. We construct the scenario $c^{*}$ by setting $c_{i^{\prime}, j}^{*}:=\hat{c}_{i^{\prime}, j}+\beta_{i^{\prime}, j}^{*} \delta_{i^{\prime}, j}$ for all $i^{\prime} \in[k]$ and $j \in[n]$ with

$$
\beta_{i^{\prime}, j}^{*}:= \begin{cases}1 & \text { for } i=i^{\prime}, j=\pi(l), 1 \leqq l \leqq \Gamma \\ 0 & \text { else. }\end{cases}
$$

Then $\sum_{i^{\prime} \in[k], j \in[n]} \beta_{i^{\prime}, j}^{*}=\Gamma$, hence $c^{*} \in \mathcal{U}^{d}$. Further, for any $\beta \in[0,1]^{k \times n}$ with $\sum_{j \in[n]} \beta_{i, j} \leqq \Gamma$ we have $\sum_{j \in[n]} \beta_{i, j} \delta_{i, j} x_{j} \leqq \sum_{j \in[n]} \beta_{i, j}^{*} \delta_{i, j} x_{j}$, because $\delta_{i, \pi(l)} x_{\pi(l), i} \leqq \delta_{i, \pi\left(l^{\prime}\right)} x_{i, \pi\left(l^{\prime}\right)}$ for $l \geqq l^{\prime}$. Consequently,

$$
z_{i}(x, c)=\sum_{j \in[n]}\left(\hat{c}_{i, j}+\beta_{i, j} \delta_{i, j}\right) x_{j} \leqq \sum_{j \in[n]}\left(\hat{c}_{i, j}+\beta_{i, j}^{*} \delta_{i, j}\right) x_{j}=z_{i}\left(x, c^{*}\right) \leqq \max _{c^{\prime} \in \mathcal{U}^{d}} z_{i}\left(x, c^{\prime}\right) \forall c \in \mathcal{U}^{\text {owb }}
$$


and therefore

$$
\max _{c \in \mathcal{U}^{\text {owb }}} \lambda_{i}\left(z_{i}(x, c)-r_{i}\right) \leqq \max _{c \in \mathcal{U}^{d}} \lambda_{i}\left(z_{i}(x, c)-r_{i}\right)
$$

Further,

$$
\max _{c \in \mathcal{U}^{d}} \lambda_{i}\left(z_{i}(x, c)-r_{i}\right) \leqq \max _{c \in \mathcal{U}^{c}} \lambda_{i}\left(z_{i}(x, c)-r_{i}\right) \leqq \max _{c \in \mathcal{U}^{\text {owb }}} \lambda_{i}\left(z_{i}(x, c)-r_{i}\right)
$$

because of $\mathcal{U}^{d} \subseteq \mathcal{U}^{c} \subseteq \mathcal{U}^{\text {owb }}$. Since these results hold for all $x \in \mathcal{X}, i \in[k]$, we get

$$
\begin{aligned}
& \max _{c \in \mathcal{U}^{d}} \lambda_{i}\left(z_{i}(x, c)-r_{i}\right)=\max _{c \in \mathcal{U}^{c}} \lambda_{i}\left(z_{i}(x, c)-r_{i}\right) \\
& =\max _{c \in \mathcal{U}^{\text {owb }}} \lambda_{i}\left(z_{i}(x, c)-r_{i}\right) \quad \forall i \in[k], x \in \mathcal{X} \\
& \Rightarrow \quad \max _{i \in[k]} \max _{c \in \mathcal{U}^{d}} \lambda_{i}\left(z_{i}(x, c)-r_{i}\right)=\max _{i \in[k]} \max _{c \in \mathcal{U}^{c}} \lambda_{i}\left(z_{i}(x, c)-r_{i}\right) \\
& =\max _{i \in[k]} \max _{c \in \mathcal{U}^{\text {owb }}} \lambda_{i}\left(z_{i}(x, c)-r_{i}\right) \quad \forall x \in \mathcal{X} \\
& \Rightarrow \quad \min _{x \in \mathcal{X}} \max _{i \in[k]} \max _{c \in \mathcal{U}^{d}} \lambda_{i}\left(z_{i}(x, c)-r_{i}\right)=\min _{x \in \mathcal{X}^{\prime}} \max _{i \in[k]} \max _{c \in \mathcal{U}^{c}} \lambda_{i}\left(z_{i}(x, c)-r_{i}\right) \\
& =\min _{x \in \mathcal{X}} \max _{i \in[k]} \max _{c \in \mathcal{U}^{\text {owb }}} \lambda_{i}\left(z_{i}(x, c)-r_{i}\right),
\end{aligned}
$$

where, again, all minima and maxima exist due to the finiteness of $\mathcal{X}$, the compactness of $\mathcal{U}^{d}, \mathcal{U}^{c}, \mathcal{U}^{\text {owb }}$ and the continuity of $z(x, \cdot):\left[\mathcal{U}^{d} / \mathcal{U}^{c} / \mathcal{U}^{\text {owb }}\right] \rightarrow \mathbb{R}$.

Because of this identity we can use the approach given in [HNS13] also for the uncertainty sets $\mathcal{U}^{d}$ or $\mathcal{U}^{c}$ :

$$
\begin{aligned}
\min _{x \in \mathcal{X}^{\prime}} \max _{c \in\left[\mathcal{U}^{\mathrm{d}} / \mathcal{U}^{\mathrm{c}} / \mathcal{U}^{\text {owb }}\right]} \max _{i \in[k]} \lambda_{i}\left(z_{i}(x, c)-r_{i}\right) & =\min _{x \in \mathcal{X}} \max _{c \in \mathcal{U}^{\text {owb }}} \max _{i \in[k]} \lambda_{i}\left(z_{i}(x, c)-r_{i}\right) \\
& =\min _{x \in \mathcal{X}} \max _{i \in[k]} \lambda_{i}\left(\max _{c \in \mathcal{U}^{\text {owb }}} z_{i}(x, c)-r_{i}\right)
\end{aligned}
$$

is equivalent to

$$
\begin{aligned}
& \min y \\
& \text { s.t. } y \geqq \lambda_{i}\left(\tilde{z}_{i}-r_{i}\right) \quad \forall i \in[k] \\
& \tilde{z}_{i} \geqq \max _{c \in \mathcal{U}^{\text {owb }}} z_{i}(x, c) \quad \forall \quad i \in[k] \\
& x \in \mathcal{X} \text {. }
\end{aligned}
$$

As shown by [BS03], the dual of the single-objective problem $\max _{c \in \mathcal{U}^{\text {owb }}} z_{i}(x, c)$ is equivalent to the linear program

$$
\begin{array}{crrr}
\min & \sum_{j \in[n]} \hat{c}_{i, j} x_{j}+\theta \Gamma+\sum_{j \in[n]} \rho_{j} & & \\
\text { s.t. } & \rho_{j}+\theta & \geqq \delta_{i, j} x_{j} & \forall j \in[n] \\
& & & \\
\theta & & & \forall j \in[n] .
\end{array}
$$


Similar to $[\mathrm{HNS13}$, we conclude that $(\mathrm{P}-\max (r, \lambda))$ is equivalent to

$$
\begin{array}{rlrl}
\min y & & & \\
\text { s.t. } & \geqq \lambda_{i}\left(\tilde{z}_{i}-r_{i}\right) & \forall i \in[k] \\
\tilde{z}_{i}-\sum_{j \in[n]} \hat{c}_{i, j} x_{j}-\theta_{i} \Gamma-\sum_{j \in[n]} \rho_{i, j} & \geqq 0 & \forall i \in[k] \\
\rho_{i, j}+\theta_{i}-\delta_{i, j} x_{j} & \geqq 0 & \forall j \in[n], i \in[k] \\
\rho_{i, j}, \theta_{i} & \geqq 0 & \forall j \in[n], i \in[k] \\
x & \in \mathcal{X} . &
\end{array}
$$

\subsubsection{MILP-formulation for $(\mathbf{P}-\min (r, \lambda))$ with continuously bounded uncertainty}

For a fixed $x$ we can reformulate $\max _{c \in \mathcal{U}^{c}} \min _{i \in[k]} \lambda_{i}\left(z_{i}(x, c)-r_{i}\right)$ as following:

$$
\begin{aligned}
& (M(x)) \max _{c \in \mathcal{U}^{c}} \min _{i \in[k]} \lambda_{i}\left(z_{i}(x, c)-r_{i}\right) \\
& \Leftrightarrow \\
& \max z \\
& \text { s.t. } \\
& z \leqq \lambda_{i}\left(\sum_{j \in[n]} \hat{c}_{i, j} x_{j}+\sum_{j \in[n]} \beta_{i, j} \delta_{i, j} x_{j}-r_{i}\right) \quad \forall i \in[k] \\
& \sum_{j \in[n], i \in[k]} \beta_{i, j} \leqq \Gamma \\
& \beta_{i, j} \in[0,1] \\
& \forall j \in[n], i \in[k]
\end{aligned}
$$

Since $\beta_{i, j}$ only contributes to the objective function if $x_{j} \neq 0$ and 0 is the only lower bound on $\beta_{i, j}$, there is always an optimal solution with $x_{j}=0 \Rightarrow \beta_{i, j}=0 \forall j \in[n], i \in[k]$. Hence, we can replace $\beta_{i, j}$ with $\tilde{\beta}_{i, j}:=\beta_{i, j} x_{j}$. Further, $\tilde{\beta}_{i, j} x_{j}=\tilde{\beta}_{i, j}$, hence we obtain the equivalent problem

$$
\begin{aligned}
\max z & & \\
\text { s.t. } & \leqq \lambda_{i}\left(\sum_{j \in[n]} \hat{c}_{i, j} x_{j}+\sum_{j \in[n]} \tilde{\beta}_{i, j} \delta_{i, j}-r_{i}\right) & \forall i \in[k] \\
\sum_{j \in[n], i \in[k]} \tilde{\beta}_{i, j} & \leqq \Gamma & \\
\tilde{\beta}_{i, j} & \leqq x_{j} & \forall j \in[n], i \in[k] \\
\tilde{\beta}_{i, j} & \geqq 0 & \forall j \in[n], i \in[k]
\end{aligned}
$$

and its dual

$$
\begin{aligned}
& (D(x)) \quad \min \sum_{i \in[k], j \in[n]} \lambda_{i} \hat{c}_{i, j} x_{j} \tau_{i}-\lambda_{i} r_{i} \tau_{i}+\Gamma \pi+\sum_{j \in[n], i \in[k]} x_{j} \nu_{i, j} \\
& \text { s.t. } \quad \sum_{i \in[k]} \tau_{i}=1 \\
& -\lambda_{i} \delta_{i, j} \tau_{i}+\pi+\nu_{i, j} \geq 0 \quad \forall j \in[n], i \in[k] \\
& \tau_{i}, \pi, \nu_{i, j}, \quad \geq 0 \quad \forall j \in[n], i \in[k]
\end{aligned}
$$


In order to use $(D(x))$ instead of $(M(x))$ as inner problem of $(\mathrm{P}-\min (r, \lambda))$, we replace $x_{j} \tau_{i}$ by the new variable $\tilde{\tau}_{i, j}$ and $x_{j} \nu_{i, j}$ by $\tilde{\nu}_{i, j}$. Since $x_{j} \in\{0,1\}, \tau_{i} \geq 0$ and $\sum_{i \in[k]} \tau_{i}=1 \Rightarrow \tau_{i} \leq 1$, we can ensure $\tilde{\tau}_{i, j}=x_{j} \tau_{i}$ by adding the constraints

$$
\begin{aligned}
& \tilde{\tau}_{i, j} \leqq \tau_{i} \\
& \tilde{\tau}_{i, j} \leqq x_{j} \\
& \tilde{\tau}_{i, j} \geqq \tau_{i}-\left(1-x_{j}\right) \\
& \tilde{\tau}_{i, j} \geqq 0 .
\end{aligned}
$$

Further, consider a feasible solution for $(D(x))$ with $\nu_{i, j}>\lambda_{i} \delta_{i, j}$. Since $\nu_{i, j}$ occurs in only one constraint, which requires

$$
\nu_{i, j} \geqq \lambda_{i} \delta_{i, j} \tau_{i}-\pi,
$$

we can choose $\nu_{i, j}=\lambda_{i} \delta_{i, j}$ instead and obtain a still feasible solution. Its objective value is not worse, since $\nu_{i, j}$ contributes with nonnegative factor to the objective function. Hence, we can restrict the feasible space of $(D(x))$ by adding the constraint $\nu_{i, j} \leqq \lambda_{i} \delta_{i, j}$. Then, the following constraints ensure that $\tilde{\nu}_{i, j}=x_{j} \nu_{i, j}$ :

$$
\begin{aligned}
& \tilde{\nu}_{i, j} \leqq \nu_{i, j} \\
& \tilde{\nu}_{i, j} \leqq x_{j} \lambda_{i} \delta_{i, j} \\
& \tilde{\nu}_{i, j} \geqq \nu_{i, j}-\lambda_{i} \delta_{i, j}\left(1-x_{j}\right) \\
& \tilde{\nu}_{i, j} \geqq 0 .
\end{aligned}
$$

We obtain the following MILP-formulation for $(\mathrm{P}-\min (r, \lambda))$ with uncertainty set $\mathcal{U}^{c}$ :

$$
\begin{aligned}
& \min \sum_{i \in[k], j \in[n]} \lambda_{i} \hat{c}_{i, j} \tilde{\tau}_{i}-\lambda_{i} r_{i} \tau_{i}+\Gamma \pi+\sum_{j \in[n], i \in[k]} \tilde{\nu}_{i, j} \\
& \text { s.t. } \quad \sum_{i \in[k]} \tau_{i}=1 \\
& -\lambda_{i} \delta_{i, j} \tau_{i}+\pi+\nu_{i, j} \geqq 0 \quad \forall j \in[n], i \in[k] \\
& \tilde{\tau}_{i, j}-\tau_{i} \leqq 0 \quad \forall j \in[n], i \in[k] \\
& \tilde{\tau}_{i, j}-x_{j} \leqq 0 \quad \forall j \in[n], i \in[k] \\
& \tilde{\tau}_{i, j}-\tau_{i}-x_{j} \geqq-1 \quad \forall j \in[n], i \in[k] \\
& \tilde{\nu}_{i, j}-\nu_{i, j} \leqq 0 \quad \forall j \in[n], i \in[k] \\
& \tilde{\nu}_{i, j}-x_{j} \lambda_{i} \delta_{i, j} \leqq 0 \quad \forall j \in[n], i \in[k] \\
& \tilde{\nu}_{i, j}-\nu_{i, j}-\lambda_{i} \delta_{i, j} x_{j} \geqq-\lambda_{i} \delta_{i, j} \quad \forall j \in[n], i \in[k] \\
& \tau_{i}, \tau_{0}, \nu_{i, j}, \tilde{\tau}_{i, j}, \tilde{\nu}_{i, j} \geqq 0 \quad \forall j \in[n], i \in[k] \\
& x \in \mathcal{X}
\end{aligned}
$$




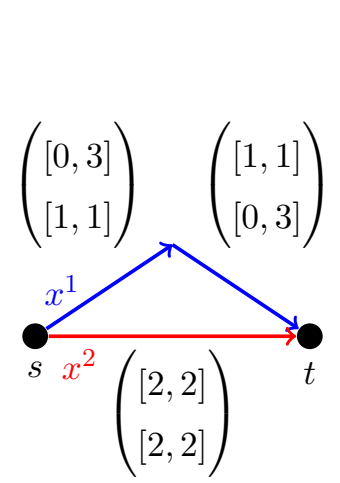

(a) Graph in Example 22

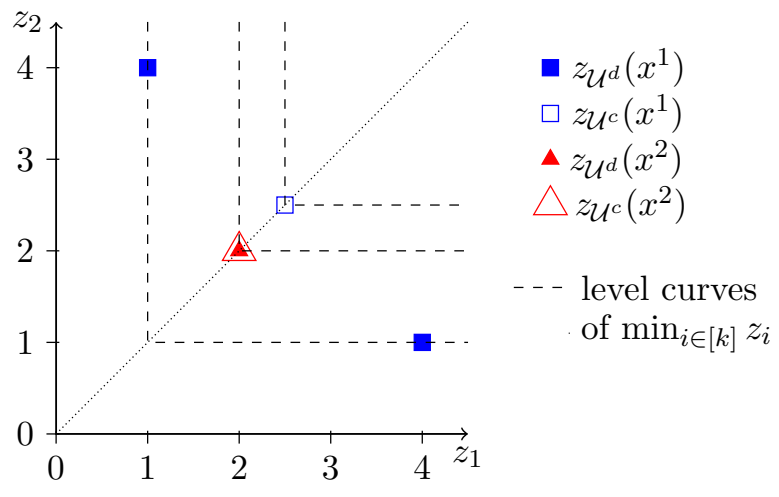

(b) $z_{\mathcal{U}^{d}}(x), z_{\mathcal{U}^{c}}(x)$ and level curves of $\min _{i \in[k]} z_{i}$

Figure 4: Example 22 shows that $(\mathrm{P}-\min (r, \lambda))$ with $\mathcal{U}^{d}$ is not equivalent to $(\mathrm{P}-\min (r, \lambda))$ with $\mathcal{U}^{c}$

\subsubsection{MILP formulation for $(P-\min (r, \lambda))$ with discretely bounded uncertainty}

In contrast to $(\mathrm{P}-\max (r, \lambda))$, the solutions for $(\mathrm{P}-\min (r, \lambda))$ with discretely bounded uncertainty can differ from the solution for $(\mathrm{P}-\min (r, \lambda))$ with continuously bounded uncertainty, as the following example shows.

Example 22. Consider an instance of $(P-\min (r, \lambda))$ with weights $\lambda=(1,1)^{T}$, reference point $r=(0,0)^{T}$, feasible set $\mathcal{X}=\left\{x^{1}=(1,1,0), x^{2}=(0,0,1)\right\}$ and discretely bounded uncertainty set $\mathcal{U}^{d}$ with $\Gamma=1$. Our nominal costs are given by $\hat{c}$ and the interval lengths are given by $\delta$ as specified below:

$$
\hat{c}=\left(\begin{array}{lll}
0 & 1 & 2 \\
1 & 0 & 2
\end{array}\right), \delta=\left(\begin{array}{lll}
3 & 0 & 0 \\
0 & 3 & 0
\end{array}\right) .
$$

The instance can for example be interpreted as an instance of the multi-objective robust shortest path problem in the graph shown in Figure 4(a).

Since only one cost value can deviate from its lower bound, we either have $z_{1}\left(x^{1}, c\right)=0+1$ or $z_{2}\left(x^{1}, c\right)=1+0$. Hence, $\max _{c \in \mathcal{U}^{d}} \min _{i \in[k]} z_{i}\left(x^{1}, c\right)=1$.

However, if we consider the continuous bounded uncertainty set $\mathcal{U}^{c}$ with the same $\Gamma, \hat{c}, \delta$ instead of $\mathcal{U}^{d}$, by setting

$$
\beta^{\prime}=\left(\begin{array}{ccc}
0 & 0.5 & 0 \\
0.5 & 0 & 0
\end{array}\right)
$$

we obtain the cost matrix

$$
c^{\prime}=\left(\begin{array}{lll}
0 & 1 & 2 \\
1 & 0 & 2
\end{array}\right)+\left(\begin{array}{ccc}
1.5 & 0 & 0 \\
0 & 1.5 & 0
\end{array}\right)=\left(\begin{array}{ccc}
1.5 & 1 & 2 \\
1 & 1.5 & 2
\end{array}\right)
$$

Therefore, $\max _{c \in \mathcal{U}^{c}} \min _{i \in[k]} z_{i}\left(x^{1}, c\right) \geq 2.5$.

On the other hand we have $\max _{c \in \mathcal{U}^{d}} \min _{i \in[k]} z_{i}\left(x^{2}, c\right)=\max _{c \in \mathcal{U}^{c}} \min _{i \in[k]} z_{i}\left(x^{2}, c\right)=2$. It follows that $x^{1}$ is the only optimal solution for $(P-\min (r, \lambda))$ with uncertainty set $\mathcal{U}^{d}$, but $x^{2}$ 
is the only optimal solution for $(P-\min (r, \lambda))$ with uncertainty set $\mathcal{U}^{c}$. The objective vectors $z(x, \xi)$ and the corresponding level curves are shown in Figure $4(b)$.

Therefore, the derived MILP-formulation for $(\mathrm{P}-\min (r, \lambda))$ with continuously bounded uncertainty is not valid for $(\mathrm{P}-\min (r, \lambda))$ with discretely bounded uncertainty. The example shows also, that the inner maximization problem of $(\mathrm{P}-\min (r, \lambda))$ is not equivalent to its linear relaxation. Hence, we cannot use the approach to dualize the linearly relaxed inner problem here. However, with help of the identity we prove in Theorem 27 we can nevertheless find a minimization problem which is equivalent to the inner maximization problem and derive a MILP formulation for $(\mathrm{P}-\min (r, \lambda))$ with discretely bounded uncertainty set.

Definition 23. Let $\delta$ be a vector in $\mathbb{R}^{n}$ or a matrix in $\mathbb{R}^{k \times l}$ and let an index set $I \subseteq[n]$ resp. $I \subseteq[k] \times[l]$ be given. We denote the $j$-smallest of all entries $\delta_{i}$ with $i \in I$ as $j-\min _{I} \delta$ and the $j$-greatest as $j-\max _{I} \delta$. For $j=0$ or $j>|I|$ we set $j-\min _{I} \delta=j-\max _{I} \delta=0$.

Notation 24. For a binary vector $x \in\{0,1\}^{n}$ we write $I(x):=\left\{j \in[n]: x_{j}=1\right\}$.

Definition 25. Let $r \in \mathbb{R}^{k}, \lambda \in \mathbb{R}_{>}^{k}$ and $x \in \mathcal{X}$ be given. We define $M \in \mathbb{R}^{k \times(\Gamma+1)}$ by its entries

$$
m_{i, l}:=\lambda_{i}\left(-r_{i}+\sum_{j \in I(x)} \hat{c}_{i, j}+\sum_{h=1}^{l-1} h-\max _{I(x)} \delta_{(i, \cdot)}\right),
$$

i.e., $\frac{m_{i, l}+r_{i}}{\lambda_{i}}$ is the sum of the nominal cost of $x$ in the $i$-th objective and the $l$ highest interval lengths $\delta_{i, j}$ among those with $x_{j}=1$ w.r.t. the $i$-th objective.

Example 26. Consider an instance of $(P-\min (r, \lambda))$ with $r=(0,0,0)^{T}, \lambda=(1,3,1)^{T}$ and uncertainty set $\mathcal{U}^{d}$ with $\Gamma=6$. Let a feasible solution $x$ be given with $|x|=6$ and

$$
\sum_{j \in I(x)} \hat{c}_{(\cdot, j)}=\left(\begin{array}{c}
10 \\
4 \\
14
\end{array}\right),\left\{\delta_{(\cdot, j)}: j \in I(x)\right\}=\left\{\left(\begin{array}{l}
2 \\
1 \\
4
\end{array}\right),\left(\begin{array}{l}
5 \\
1 \\
3
\end{array}\right),\left(\begin{array}{l}
1 \\
1 \\
5
\end{array}\right),\left(\begin{array}{l}
0 \\
1 \\
2
\end{array}\right),\left(\begin{array}{l}
3 \\
1 \\
6
\end{array}\right),\left(\begin{array}{l}
4 \\
1 \\
1
\end{array}\right)\right\} .
$$

We exemplarily compute $m_{1,3}$. For the first objective, the highest interval length $\delta_{1, j}$ among those with $j \in I(x)$ is 5 , the second highest 4 and the nominal cost 10 . Hence, we obtain

$$
m_{1,3}=\lambda_{1}\left(-r_{1}+\sum_{j \in I(x)} \hat{c}_{1, j}+\sum_{h=1}^{2} h-\max _{I(x)} \delta_{(1, \cdot)}\right)=1(-0+10+5+4)=19 .
$$

The complete matrix for this example is

$$
M=\left(\begin{array}{lllllll}
10 & 15 & 19 & 22 & 24 & 25 & 25 \\
12 & 15 & 18 & 21 & 24 & 27 & 30 \\
14 & 20 & 25 & 29 & 32 & 34 & 35
\end{array}\right)
$$


Theorem 27. Given $x \in \mathcal{X}$ and the corresponding matrix $M$, the optimal objective value $z^{*}$ of the inner maximization problem of $(P-\min (r, \lambda))$ equals the $(\Gamma+1)$-smallest entry in $M$, i.e.,

$$
z^{*}:=\max _{c \in \mathcal{U}} \min _{i \in[k]} \lambda_{i}\left(z_{i}(x, c)-r_{i}\right)=(\Gamma+1)-\min _{[k] \times[\Gamma+1]} M=: m^{*}
$$

Proof. We show first, that $z^{*} \leqq m^{*}$. Let $c^{*}$ with $c_{i, j}^{*}=\hat{c}_{i, j}+\beta_{i, j}^{*} \delta_{i, j}$ be an optimal solution of the inner maximization problem $\max _{c \in \mathcal{U}} \min _{i \in[k]} \lambda_{i}\left(z_{i}(x, c)-r_{i}\right)$ with objective value $z^{*}$. Let us now look at the structure of the $\operatorname{cost}$ matrix $c^{*}$, or, more precisely, at each row $c_{(i,)}^{*}$ of this matrix, representing the costs under objective $i$ in scenario $c^{*}$. Let $l_{i}:=\sum_{j \in[n]} \beta_{i, j}^{*}$ be the number of entries in this row which deviate from their nominal value. Since we maximize the costs we can w.l.o.g. assume that among all $i \in I(x)$ the $l_{i}$ indices with highest entries in $\delta_{(i, \cdot)}$ are chosen to deviate from the nominal value.

Due to the construction of the matrix $M$, it follows that the objective value of $x$ in scenario $c^{*}$ with respect to objective $i$ is equal to the $\left(l_{i}+1\right)$ st entry of line $m_{i, \text { : }}$

$$
\lambda_{i}\left(z_{i}\left(x, c^{*}\right)-r_{i}\right)=\lambda_{i}\left(-r_{i}+\sum_{j \in I(x)} \hat{c}_{i, j}+\sum_{h=1}^{l_{i}} h-\max _{I(x)} \delta_{(i, \cdot)}\right)=m_{i,\left(l_{i}+1\right)} .
$$

$M$ is constructed such that in each row $i$ we have $m_{i, l} \leqq m_{i, l^{\prime}} \forall l \leqq l^{\prime}$. Hence, in row $i$ there are at most $l_{i}$ matrix entries smaller than $m_{i, l_{i}+1}$ and in total there are at most $\sum_{i \in[k]} l_{i}=\sum_{i \in[k]} \sum_{j \in[n]} \beta_{i, j}^{*} \leqq \Gamma$ matrix entries smaller than $\min _{i \in[k]} m_{i, l_{i}+1}$. This implies

$$
z^{*}=\min _{i \in[k]} m_{i,\left(l_{i}+1\right)} \leqq(\Gamma+1)-\min _{i \in[k], j \in[\Gamma+1]} M=m^{*} .
$$

To show $z^{*} \geqq m^{*}$, we construct a scenario $\tilde{c} \in \mathcal{U}$ with objective value $m^{*}$. For each $i \in[k]$ we define

$$
\hat{l}_{i}:=\max \left\{l: m_{i, l}<m^{*}\right\} .
$$

Because of $m_{i, l} \leqq m_{i, l^{\prime}} \forall l \leqq l^{\prime}$, we have $m_{i, l}<m^{*} \forall l \leqq \hat{l}_{i}$ and $m^{*} \leqq m_{i,\left(\hat{l}_{i}+1\right)} \leqq m_{i, l^{\prime}} \forall l^{\prime}>\hat{l}_{i}$ we conclude

$$
\sum_{i=1}^{k} \hat{l}_{i} \leqq \Gamma \text { and } m^{*}=\min _{i \in[k]} m_{i,\left(\hat{l}_{i}+1\right)}
$$

We construct a $\tilde{\beta}$ such that the solution $\tilde{c}$ with $\tilde{c}_{i, j}=\hat{c}_{i, j}+\tilde{\beta}_{i, j} \delta_{i, j}$ is feasible and has objective value $m^{*}$ : For each $i \in[k]$ we choose a set $\hat{J}_{i} \subseteq I(x)$ of $\hat{l}_{i}$ indices with largest interval lengths, i.e., such that $\left|\hat{J}_{i}\right|=\hat{l}_{i}$ and $\delta_{i, j} \geqq \delta_{i, j^{\prime}} \forall j \in \hat{J}_{i}, j^{\prime} \in I(x) \backslash \hat{J}_{i}$. We set

$$
\tilde{\beta}_{i, j}:=\left\{\begin{array}{ll}
1 & \text { for } j \in \hat{J}_{i} \\
0 & \text { else }
\end{array} \quad \text { and } \quad \tilde{c}_{i, j}:=\hat{c}_{i, j}+\tilde{\beta}_{i, j} \delta_{i, j} .\right.
$$


Then $\sum_{i \in[k], j \in[n]} \tilde{\beta}_{i, j}=\sum_{i \in[n]} \hat{l}_{i} \leqq \Gamma$, hence, $\tilde{c} \in \mathcal{U}^{d}$. Further,

$$
\begin{aligned}
z^{*} & \geqq \min _{i \in[k]} \lambda_{i}\left(z_{i}(x, \tilde{c})-r_{i}\right)=\min _{i \in[k]} \lambda_{i}\left(\sum_{j \in[n]}\left(\hat{c}_{i, j} x_{j}+\tilde{\beta}_{i, j} \delta_{i, j} x_{j}\right)-r_{i}\right) \\
& =\min _{i \in[k]} \lambda_{i}\left(-r_{i}+\sum_{j \in[n]} \hat{c}_{i, j} x_{j}+\sum_{j \in \hat{J}_{i}} \delta_{i, j}\right) \\
& =\min _{i \in[k]} \lambda_{i}\left(-r_{i}+\sum_{j \in[n]} \hat{c}_{i, j} x_{j}+\sum_{h=0}^{\hat{l}_{i}} h-\max _{I(x)} \delta_{(i, \cdot)}\right)=\min _{i \in[n]} m_{i,\left(\hat{l}_{i}+1\right)}=m^{*} .
\end{aligned}
$$

Example 28. Consider the instance in Example 26 and the feasible solution $x$. We have $\Gamma+1=7$ and the 7 -th smallest entry in $M$ is 19. It follows that $\max _{c \in \mathcal{U}^{d}} \min _{i \in[k]} \lambda_{i}\left(z_{i}(x, c)-\right.$ $\left.r_{i}\right)=19$.

With help of this equality we derive a MILP formulation for $(\mathrm{P}-\min (r, \lambda))$. In a preprocessing step, for each $i \in[k]$ we sort the entries of the vector $\delta_{(i, \cdot)}$ decreasingly and set

$$
y_{i, j, j^{\prime}}:= \begin{cases}1 & \text { if } \delta_{i, j} \text { before } \delta_{i, j^{\prime}} \text { w.r.t. this sorting } \\ 0 & \text { else }\end{cases}
$$

Then, for a given $x$, we can formulate $\max _{\xi \in \mathcal{U}} \min _{i \in[k]} \lambda_{i}\left(z_{i}(x, c)-r_{i}\right)$ as a minimization problem with the variables

$$
\begin{array}{ll}
z & \text { being the objective value } \\
m_{i, l} \quad \text { representing } m_{i, l} \text { as given in Definition } 25 \\
w_{i, l} \quad \text { indicating if } m_{i, l} \text { is one of the } \Gamma+1 \text { smallest entries of } M \\
u_{i, j, l} \quad \text { indicating if } \delta_{i, j} \text { is one of the summands in } m_{i, l} \\
q_{l} \quad \text { indicating if } x \text { contains at least } l \text { elements }
\end{array}
$$

and the constants

$$
N_{i}:=\sum_{j \in[n]}\left(\hat{c}_{i, j}+\delta_{i, j}\right) \forall i \in[k] .
$$

If $x$ is known, many of the values can be precomputed. However, when using the problem as inner problem for $(\mathrm{P}-\min (r, \lambda))$, they are variables. We construct the following MILP 
formulation for $\max _{\xi \in \mathcal{U}} \min _{i \in[k]} \lambda_{i}\left(z_{i}(x, c)-r_{i}\right)$ :

$$
\begin{aligned}
& \min \quad z \\
& \text { s.t. } \quad z \geqq m_{i, l}-\left(1-w_{i, l}\right) N_{i} \quad \forall i \in[k], l \in[\Gamma+1] \\
& \sum_{\substack{i \in[k] \\
l \in[\Gamma+1]}} w_{i, l}=\Gamma+1 \\
& m_{i, l}=\lambda_{i}\left(\sum_{j \in[n]} \hat{c}_{i, j} x_{j}+\sum_{j \in[n]} u_{i, j, l} \delta_{i, j}-r_{i}\right) \quad \forall i \in[k], l \in[\Gamma+1] \\
& \sum_{j \in[n]} u_{i, j, l} \geqq(l-1)-\Gamma q_{l} \quad \forall i \in[k], l \in[\Gamma+1] \\
& \sum_{j \in[n]} u_{i, j, l} \geqq \sum_{j \in[n]} x_{j}-|E|\left(1-q_{l}\right) \quad \forall i \in[k], l \in[\Gamma+1] \\
& u_{i, j, l} \leqq x_{j} \\
& u_{i, j^{\prime}, l}-u_{i, j, l} \leqq 1-y_{i, j, j^{\prime}} x_{j} \\
& \forall j \in[n], i \in[k], l \in[\Gamma+1] \\
& \forall j, j^{\prime} \in[n], i \in[k], l \in[\Gamma+1] \\
& \forall j \in[n], i \in[k], l \in[\Gamma+1]
\end{aligned}
$$

The first two constraints ensure that $z$, when minimized, is set to the $(\Gamma+1)$-smallest of the variables $m_{i, l}$. Because of Constraints (4) and (5), for each $i$ and $l$ at least $\min \{|x|, l-1\}$ of the $u_{i, j, l}$ are set to 1 . Hence, at least $\min \{|x|, l-1\}$ of the $\delta_{i, j}$ are summed up in Constraint (3). Constraints (6) and (7) ensure, that these are the largest $\delta_{i, j}$ among those with $x_{j}=1$. We obtain

$$
\sum_{h=1}^{l-1} h-\max _{I(x)} \delta_{(i, \cdot)} \leq \sum_{j \in[n]} u_{i, j, l} \delta_{i, j} \forall l \in[\Gamma+1], i \in[k],
$$

with equality in case of an optimal solution, because $z$ is minimized, hence $m_{i, l}$ is minimized. Then, $m_{i, l}$ take exactly the values given in Definition 25 (Constraint (3)). We conclude that $(\mathrm{P}-\min (r, \lambda))$ with uncertainty set $\mathcal{U}^{d}$ can be formulated as

$$
\begin{array}{lll}
(\mathrm{P}-\min (r, \lambda)) & \min & z \\
& \text { s.t. } & (1)-(8) \\
& & x \in \mathcal{X} .
\end{array}
$$

\subsubsection{Complexity of $(\mathbf{P}-\min (r, \lambda))$ and $(\mathbf{P}-\max (r, \lambda))$ with bounded uncertainty}

For $\Gamma=0$, the uncertainty sets $\mathcal{U}^{d}$ and $\mathcal{U}^{c}$ only contain one scenario. From Remark 9 it hence follows, analogous to the case of interval uncertainty, that $(\mathrm{P}-\min (r, \lambda))$ is polynomially solvable, if the single-objective deterministic problem is polynomially solvable, whereas $(\mathrm{P}-\max (r, \lambda))$ is NP-hard for several combinatorial problems, e.g., the shortest path, minimum spanning tree and assignment problem.

The following Theorem shows that $(\mathrm{P}-\min (r, \lambda))$ with uncertainty set $\mathcal{U}^{d}$ is NP-hard for the shortest path and minimum spanning tree problem, if $\Gamma=1$. 
Theorem 29. $(P-\min (r, \lambda))$ with uncertainty set $\mathcal{U}^{d}$ and $\Gamma=1$ is NP-hard for the shortest path problem and the minimum spanning tree problem, even for two objectives, $\lambda=(1,1)^{T}$ and $r=(0,0)^{T}$.

Proof. We consider the single-objective minmax robust shortest path resp. minimum spanning tree problem with a discrete scenario set consisting of two scenarios. This has been proven to be NP-hard for both problems (see [KY97]). We reduce it to (P-min $(r, \lambda)$ ) with two objectives and discretely bounded uncertainty set with $\Gamma=1$.

Let an instance $I$ of the single-objective minmax robust problem be given. In case of the shortest path problem, we have given a graph $G$ with edge set $E=\left\{e_{1}, \ldots, e_{n}\right\}$, and a start node $s$ and end node $t$ in $G$. The set of feasible solutions $\mathcal{X} \subseteq\{0,1\}^{n}$ contains all vectors that represent a simple path from $s$ to $t$. In case of the minimum spanning tree problem, $E$ is again the edge set of a graph $G$ and the feasible solutions represent the spanning trees in $G$. Further, we have given two scenarios $\xi^{1}, \xi^{2}$ and edge costs $b \in \mathbb{R}^{2 \times n}$, assigning cost $b_{i, j}$ to edge $e_{j}$ under scenario $\xi$. We construct an instance $I^{\prime}$ of $(\mathrm{P}-\min (r, \lambda))$ as following:

- We start with the graph $G$ from $I$ and construct edge costs for the discretely bounded uncertainty set: $\hat{c}_{i, j}:=b_{i, j}, \delta_{i, j}=0 \forall j \in[n], i \in[2]$.

- We then add one new node $s^{\prime}$ and one new edge $e_{n+1}$ : For the minimum spanning tree problem, $e_{n+1}$ connects $s^{\prime}$ to any of the other nodes. For the shortest path problem, the edge $e_{n+1}$ leads from $s^{\prime}$ to the original start node $s$.

- We construct cost intervals for the new edge: For some upper bound $B \geq \max _{i=1,2} \sum_{j \in[n]} \hat{c}_{i, j}$ we define $\hat{c}_{(\cdot, n+1)}:=(0,0)^{T}, \delta_{(\cdot, n+1)}:=(B, B)^{T}$.

- We define the new feasible set $\mathcal{X}^{\prime}:=\left\{\left(\begin{array}{l}x \\ 1\end{array}\right): x \in \mathcal{X}\right\}$.

Note, that in case of the spanning tree problem, $\mathcal{X}^{\prime}$ represents the set of all spanning trees in the new graph, since the only edge connecting $s^{\prime}$ to the old graph is $e_{n+1}$. In case of the robust shortest path problem, $\mathcal{X}^{\prime}$ represents the set of all paths from the new node $s^{\prime}$ to the original destination node $t$ in the new graph, because $s^{\prime}$ has exactly one outgoing edge $e_{n+1}$, which ends in the original start node $s$.

Constructed like this, for every $x \in \mathcal{X}$ the solution $x^{\prime}:=(x, 1)^{T}$ is feasible for $I^{\prime}$ and for every $x^{\prime} \in \mathcal{X}^{\prime}$, the solution $x:=\left(x_{1}^{\prime}, \ldots, x_{n}^{\prime}\right)^{T}$ is feasible for $I$. Hence, every feasible solution $x$ for $I$ corresponds to a feasible solution $x^{\prime}$ for $I^{\prime}$ and vice versa.

Since for every $x^{\prime} \in \mathcal{X}^{\prime}$ we have $x_{(n+1)}^{\prime}=1$, its worst case scenario is either

$$
\begin{aligned}
& c^{1}: c_{1,(n+1)}^{1}=B, c_{1,(n+1)}^{2}=0, c_{i, j}^{1}=\hat{c}_{i, j} \forall j \neq n+1 \text { or } \\
& c^{2}: c_{1,(n+1)}^{1}=0, c_{2,(n+1)}=B, c_{i, j}=\hat{c}_{i, j} \forall j \neq n+1,
\end{aligned}
$$

because all other feasible scenarios are equivalent to just considering the nominal edge lengths (since $\Gamma=1)$. The choice of $B$ ensures $z_{1}\left(x^{\prime}, c^{1}\right) \geqq z_{2}\left(x^{\prime}, c^{1}\right)$ and $z_{2}\left(x^{\prime}, c^{2}\right) \geqq z_{1}\left(x^{\prime}, c^{2}\right)$ for all 
$x^{\prime} \in \mathcal{X}^{\prime}$. It follows that for every $x^{\prime} \in \mathcal{X}^{\prime}$

$$
\begin{aligned}
\max _{c \in \mathcal{U}} \min _{i=1,2} z_{i}\left(x^{\prime}, c\right) & =\max \left\{\min \left\{z_{1}\left(x^{\prime}, c^{1}\right), z_{2}\left(x^{\prime}, c^{1}\right)\right\}, \min \left\{z_{1}\left(x^{\prime}, c^{2}\right), z_{2}\left(x^{\prime}, c^{2}\right)\right\}\right\} \\
& =\max \left\{z_{2}\left(x^{\prime}, c^{1}\right), z_{1}\left(x^{\prime}, c^{2}\right)\right\}=\max \left\{\sum_{j \in[n]} \hat{c}_{2, j} x_{j}^{\prime}, \sum_{j \in[n]} \hat{c}_{1, j} x_{j}^{\prime}\right\} \\
& =\max _{i=1,2} \sum_{j \in[n]} \hat{c}_{i, j} x_{j}^{\prime}=\max _{i=1,2} \sum_{j \in[n]} b_{i, j} x_{j}^{\prime} .
\end{aligned}
$$

We conclude that an optimal solution for $I^{\prime}$ corresponds to an optimal solution for $I$ and vice versa.

\section{Conclusion}

In this paper we introduced two methods to find minmax robust efficient solutions based on scalarizations: the min-ordering and the max-ordering method. We have shown that the max-ordering method finds (all) point-based minmax robust weakly efficient solutions. The min-ordering solution finds set-based minmax robust weakly efficient solutions, which cannot necessarily be found with scalarization based methods for multi-objective robust optimization from the literature.

We investigated the resulting scalarized problems $(\mathrm{P}-\min (r, \lambda))$ and $(\mathrm{P}-\max (r, \lambda))$ for multiobjective combinatorial problems with particular uncertainty sets. For interval uncertainty we could show that only one scenario needs to be considered. Then, $(\mathrm{P}-\max (r, \lambda))$ reduces to a single-objective minmax robust problem with discrete uncertainty set, whereas a solution to $(\mathrm{P}-\min (r, \lambda))$ can be found by solving several single-objective deterministic problems with the same feasible set. We further extended the single-objective concept of bounded uncertainty to the multi-objective case. We developed MILP-formulations for both $(\mathrm{P}-\min (r, \lambda))$ and $(\mathrm{P}-\max (r, \lambda))$ with bounded uncertainty and investigated the complexity of the resulting problems.

The first question in mind for further investigations is, how to solve $(\mathrm{P}-\mathrm{min}(r, \lambda))$ and $(\mathrm{P}-\max (r, \lambda))$ in case of multi-objective robust combinatorial problems with other uncertainty sets, e.g., discrete scenarios sets or polyhedral or ellipsoidal uncertainty. Also, the complexity of $(\mathrm{P}-\min (r, \lambda))$ with uncertainty set $\mathcal{U}^{c}$ remains an open question.

Further research could be done on specialized solution approaches for particular combinatorial problems, for example the shortest path or minimal spanning tree problem. It is also interesting to check if solutions to other robustness concepts, e.g., hull-based minmax robust efficiency [BF17], multi-scenario efficiency [BS16], or lightly robust efficiency [IS16] can be found with the min-ordering or max-ordering method.

A variant of the max-ordering or min-ordering optimization problem is to look for the second/third/... highest or smallest objective instead of the maximum or minimum. Moreover, we have shown that the solutions of $(\mathrm{P}-\min (r, \lambda))$ and $(\mathrm{P}-\max (r, \lambda))$ have quite different properties and characterizations. It would therefore also be of interest to consider a combination of both by choosing any ordered median function as scalarizing function and analyze the resulting problems. 


\section{Acknowledgments}

Lisa Thom was supported by DFG RTG 1703 "Resource Efficiency in Interorganizational Networks".

\section{References}

[BF17] R. Bokrantz and A. Fredriksson. Necessary and sufficient conditions for Pareto efficiency in robust multiobjective optimization. European Journal of Operational Research, 262(2):682-692, 2017.

[BS03] D. Bertsimas and M. Sim. Robust discrete optimization and network flows. Mathematical Programming, 98(1):49-71, 2003.

[BS16] M. Botte and A. Schöbel. Dominance for multi-objective robust optimization. Technical Report 2016-8, Preprint-Reihe, Institut für Numerische und Angewandte Mathematik, Universität Göttingen, 2016.

[BTEGN09] A. Ben-Tal, L. El Ghaoui, and A. Nemirovski. Robust optimization. Princeton University Press, Princeton and Oxford, 2009.

[BTGGN04] A. Ben-Tal, A. Goryashko, E. Guslitzer, and A. Nemirovski. Adjustable robust solutions of uncertain linear programs. Mathematical Programming, 99(2):351376, 2004.

[BTN98] Aharon Ben-Tal and Arkadi Nemirovski. Robust convex optimization. Mathematics of Operations Research, 23(4):769-805, 1998.

[DKW12] E.K. Doolittle, H.L. M. Kerivin, and M. M. Wiecek. A robust multiobjective optimization problem with application to internet routing. Technical Report R2012-11-DKW, Clemson University, 2012.

[Ehr06] M. Ehrgott. A discussion of scalarization techniques for multiple objective integer programming. Annals of Operations Research, 147(1):343-360, 2006.

[EIS14] M. Ehrgott, J. Ide, and A. Schöbel. Minmax robustness for multi-objective optimization problems. European Journal of Operational Research, 239(1):1731, 2014.

[FW14] J. Fliege and R. Werner. Robust multiobjective optimization \& applications in portfolio optimization. European Journal of Operational Research, 234(2):422433, 2014.

[HNS13] F. Hassanzadeh, H. Nemati, and M. Sun. Robust optimization for multiobjective programming problems with imprecise information. Procedia Computer Science, 17:357-364, 2013.

[Ide14] J. Ide. Concepts of Robustness for Uncertain Multi-Objective Optimization. PhD thesis, Universität Göttingen, 2014. 
[IS16] J. Ide and A. Schöbel. Robustness for uncertain multi-objective optimization: a survey and analysis of different concepts. OR Spectrum, 38(1):235-271, 2016.

[KL12] D. Kuroiwa and G. M. Lee. On robust multiobjective optimization. Vietnam Journal of Mathematics, 40(2-3):305-317, 2012.

[KRSS16] K. Kuhn, A. Raith, M. Schmidt, and A. Schöbel. Bi-objective robust optimisation. European Journal of Operational Research, 252(2):418-431, 2016.

[KY97] P. Kouvelis and G. Yu. Robust discrete optimization and its applications. Kluwer Academic Publishers, Boston, 1997.

[RSST17] A. Raith, M. Schmidt, A. Schöbel, and L. Thom. Extensions of labeling algorithms for multi-objective uncertain shortest path problems. Technical Report 2017-2, Preprint-Reihe, Institut für Numerische und Angewandte Mathematik, Universität Göttingen, 2017.

[RSST18] A. Raith, M. Schmidt, A. Schöbel, and L. Thom. Multi-objective minmax robust combinatorial optimization with cardinality-constrained uncertainty. European Journal of Operational Research, 267(2):628-642, 2018.

[Soy73] A. L. Soyster. Technical note-Convex programming with set-inclusive constraints and applications to inexact linear programming. Operations Research, 21(5):1154-1157, 1973.

[WD16] M. M. Wiecek and G. M. Dranichak. Robust multiobjective optimization for decision making under uncertainty and conflict. In A. Gupta and A. Capponi, editors, Optimization Challenges in Complex, Networked and Risky Systems, INFORMS Tutorials in Operations Research (J.C. Smith, ed.), chapter 4, pages 84-114. INFORMS, 2016.

$\left[W_{L}+17\right] \quad$ L. Wang, Q. Li, R. Ding, M. Sun, and G. Wang. Integrated scheduling of energy supply and demand in microgrids under uncertainty: A robust multi-objective optimization approach. Energy, 130:1 - 14, 2017. 



\section{Danke}

Als erstes möchte ich meiner Doktormutter Anita Schöbel danken: Mit deiner Begeisterung für Mathematik und deiner optimistischen Art hast du mich immer wieder aufs Neue motiviert. Danke für deine Unterstützung und deinen hilfreichen Rat und dafür, dass du trotz deiner vielen Aufgaben immer ansprechbar warst und dir Zeit für meine Anliegen genommen hast!

Bei ihr und meinen beiden anderen Koautorinnen, Andrea Raith und Marie Schmidt, bedanke ich mich außerdem besonders für die gute Zusammenarbeit und die spannenden und ertragreichen Diskussionen. Marie, vielen Dank für die fröhlichen und produktiven Stunden, die wir gemeinsam in Göttingen und Rotterdam verbracht haben, und dafür, dass du dich bereit erklärt hast, meine Arbeit zu begutachten! Andrea, dir möchte ich besonders für die Einladung nach Auckland und unsere Zusammenarbeit dort danken, und für deinen Einsatz für unser Labeling-Paper.

Mein Dank geht auch an meine Zweitbetreuerin Anja Fischer: Danke für deine Hilfsbereitschaft, deine guten Ideen und dein Lachen!

Für die Finanzierung meiner Stelle, meiner Forschungsaufenthalte und Konferenzbesuche bin ich der DFG und dem Graduiertenkolleg 1703 zu Dank verpflichtet. Ich danke auch den Mitgliedern des Kollegs für die Gemeinschaft und den Wissensaustausch, insbesondere der Leiterin Jutta Geldermann, Meike und Kristin für Rat und Tat und ihr Engagement, und Willy für unsere interdisziplinäre Zusammenarbeit.

Ganz besonders möchte ich mich bei meiner Arbeitsgruppe bedanken: für die Gemeinschaft und die angenehme Arbeitsatmosphäre, für viele gemeinsame Mittagessen und selbstgebackene Kuchen, für Beistand in technischen, mathematischen und anderen Fragen des Lebens, für Chorgesang und gemeinsames Forschen. Danke, Alex, Anita, Anja, Corinna, Jörn, Jonas, Julius, Marco Be., Marco Bo., Mirko, Morten, Philine, Robert, Sebastian und Sönke! Ich danke auch Fabian für seine Programmierarbeit und unserem inoffiziellen AG-Mitglied Emelie, deren Lächeln Herzen öffnet.

Ich danke allen Freunden, die mich in meiner Doktorandenzeit unterstützt haben, sei es durch ein offenes Ohr für meine Sorgen oder ihr Ablenkungsvermögen. Besonderer Dank geht an Charlotte und Philine für das Korrekturlesen meiner Arbeit! Mit ihrer Zuversicht und bedingungslosen Unterstützung hat mir meine Familie in dieser Zeit sehr geholfen. Mama, Papa und Oma, danke, dass ihr für mich da seid! Hauke, dir gebührt besonderer Dank für deine Geduld, deinen guten Rat und dein offenes Ohr (auch morgens um fünf) und dafür, dass du es immer schaffst, die Welt ein wenig heller aussehen zu lassen. Es tut gut, dich an meiner Seite zu wissen. 
